$\mid+1 n^{2}+1$

$$
=0
$$

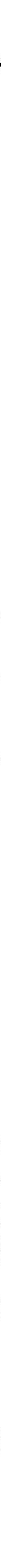

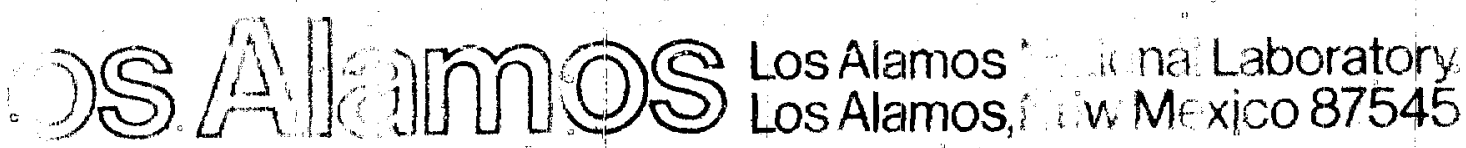

0 
UC. 4

Issued: September 1981

\title{
Quality Assurance for Environmental \\ Analytical Chemistry: 1980
}

\author{
Ernest S. Gladney \\ William E. Goode \\ Daniel R. Perrin \\ Colleen E. Burns
}

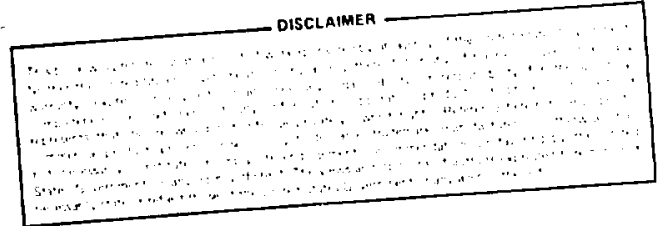

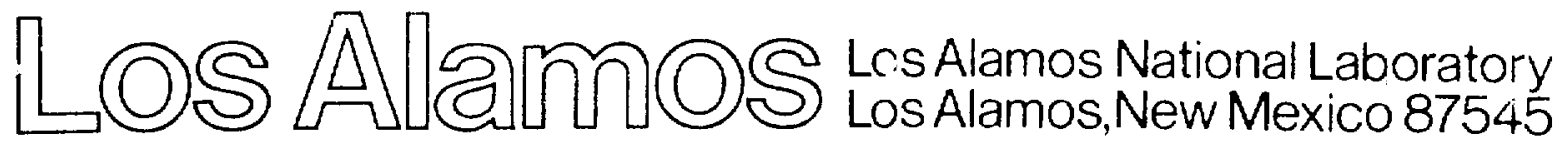




\title{
QUALITY ASSURANCE FOR ENVIRONMENTAL ANALYTICAL CHEMISTRY: 1980
}

by

Ernest S. Gladney, William E. Goode, Daniel R. Perrin, and Colleen E. Burns

\begin{abstract}
The continuing quality assurance effort by the Environmental Surveillance Group is presented. Inchuded are all standard materials now in use, their consensus or certified concentrations, quality control charts, and all quality assurance measurements made by H-8 during 1900.
\end{abstract}

\section{INTRONUCTION AND DEFINITIONS}

This is the second in an annual series of analytical quality assurance documents presenting quality assurance data for environmental analyses performed by the Environmental Surveillance Group (H-8) during CY 1980. The philosophy, methodolosy, and computer support programs utilized have been described previously.' There have been no important changes in these areas this year.

Most terms, acronyms, and abbreviations used in this document have been defined previously. ${ }^{1}$ Part of this list is repeated below and includes a few updates.

ASTM. American Society for Testing Materials.

Average (Mean). The sum of a series of results divided by the number of results. Symbol is $\bar{x}$.

CCRMP. Canadian Certified Reference Materials Program.

CGS. Canadian Geological Survey.

CRPG. Centre de Recherches Petrographiques et Geochimiques de Nancy.
EML. Environmental Measurements Laboratory, New York, USA.

EMSL. Environmental Monitoring ad Support Laboratory, EPA, Cincinnati, Ohio.

EMSL-LV. Environmental Monitoring and Suppuri Laboratory, EPA, Las Vegas, Nevada.

EPA. Environmental Protection Agency, ISSA.

JDEN. Junta de Energia Nuclear, Madrid, Spain.

H-8. Environmental Surveillance Group, Los Alamos National Laboratory.

IAEA. International Atomic Energy Agency.

NBS. National Burcau of Standards, USA.

Primary Standards. A class of single elements or compounds that are well-characterized, stable, homogeneous, and produced in quantity. Their physical or chemical properties have been determined within specified measurement uncertainties by a recoznized national standards laboratory such as NBS or NPL. NBS SRM 83c $\left(\mathrm{As}_{2} \mathrm{O}_{3}\right)$ and NBS SRM 950 $\left(\mathrm{U}_{3} \mathrm{O}_{2}\right)$ are examples. 
Quality Assurance (QA). Planned and systematic actions necessary to provide confidence in each analytical result reported by a laboratory.

Quality Control (QC). Actions taken within a quality assurance program to ensure validity of results. Quality control as used in this document means calibration of equipment and. when possible, control of a method through use of secondary standards to develop control charts.

Quality Control Charts. A control chart consists of a graph with the ordinate scaled in units of the test result and the abscissa scaled in units of time or sequence of results. Upper and lower control limits on the chart are used as criteria for action or for judging the significance of variations between duplicate samples. The centerline represents the average or standard value of the statistical measure being plotted.

Range (R). The absolute value of the difference be tween the highest and the lowest values in a set of results

Secondary Standards. Materials produced by commercial organizations, individual laboratories, or governmental agencies that are well-characterized, stable, and homogeneous. They are produced in quantity and have one or more physical or chemical properties experimentally determined within stated measurement uncertainties. The important distinction between primary and secondary standards is that the latter may be complex natural materials (rocks, vegetation, etc.) as well as high purity compounds or elements.

We differentiate Secondary Standards into two classes.

Class I: Complex materials whose composition or physical properties have been determined and certified within specified measurement uncertainties by a recognized national standards laboratory. The NBS Standard Reference Materials program has produced a large number of such materials for use in environmental analysis.

Class II: Complex materials whose preparation has been similar to that of Class I materials, but whose composition has not been certified by a national standards laboratory. Certain elements in NBS
SRMs, and all materials from the USGS, CCRMP. IAEA, or EPA fall in this category. Mean elemental concentrations in these materials are frequently established by consensus. ${ }^{1-19}$

SRM. Standard Reference Material.

Standard Deviation. A statistical measure of data variability. Symbol is $\mathbf{S}$.

Traceability. A hierarchical system, used largely for regulatory purposes, wherein transfer of accuracy among various components of a measurement system is established. For example, a working standard should be demonstrated to be traceable to a secondary standard, Class II secondary standards to Class I secondary standards, and secondary standards to primary standards. See Uriano and Gravatt for more details. ${ }^{16}$

Working Standards. Materials produced by commercial organizations or by individual laboratories for direct use in instrument calibration. These are generally single elements in solution. A good suggested list of materials for working standards can be found in Gladney et al. ${ }^{17}$

\section{QUALITY ASSURANCE DATA FOR 1980}

The current selection of Class I and Class II secondary standards available to $\mathrm{H}-8$ is shown in Table I. Our Laboratory has adopted a sample numbering scheme that uses a number of the structure AA.xxxxx where AA are the last two digits of the calendar year (e.g., 80) and $\mathbf{x x x x x}$ is a five-digit number serially assigned in blocks to various customers. A certain bluck of serial numbers is reserved for the $Q A$ samples (AA.00500-AA.00799) and reused yearly changing only the calendar year designator AA. The abbreviations A, B, M, S, V, and W, stand for air, biological, metal, rock (usually silicate), vegetation, and water, respectively. Current certified values and "best" consensus values for elemental concentrations in each secondary standard are listed in Appendix A. Since matrix independence is difficult or impossible for many analytical techniques, a large set of secondary standards is maintained. We draw upon NBS, EPA, USGS, CCRMP, and IAEA for secondary reference materials. 
TABLE 1

\section{QUALITY ASSURANCE MATERIALS IN H-8 LIBRARY}

\begin{tabular}{|c|c|c|c|}
\hline $\begin{array}{c}\text { Suniple } \\
\text { Number } \\
\end{array}$ & $\begin{array}{c}\text { Sample } \\
\text { Type } \\
\end{array}$ & $\begin{array}{c}\text { Date } \\
\text { Received } \\
\end{array}$ & $\begin{array}{c}\text { Sample } \\
\text { Name }\end{array}$ \\
\hline 80.00500 & $\mathbf{w}$ & $80 / 03 / 20$ & H-8 TDS \\
\hline 80.00501 & $\mathbf{w}$ & $80 / 03 / 20$ & H-8 Conductivity \\
\hline 80.00502 & $\mathbf{S}$ & $80 / 04 / 16$ & NBS 4350 Environmental Radioactivity Sediment \\
\hline 80.00503 & W & $80 / 04 / 25$ & EPA Nitrate/Fluoride-1 \\
\hline 80.00504 & $w$ & $80 / 04 / 25$ & EPA Nitrate/Fluoride-2 \\
\hline 80.00505 & $w$ & $80 / 04 / 14$ & NBS 1643A Water \\
\hline 80.00506 & $\mathbf{S}$ & $80 / 01 / 01$ & USGS BIR-1 \\
\hline 80.00507 & $S$ & $80 / 01 / 01$ & USGS DNC-1 \\
\hline 80.00508 & S & $80 / 01 / 01$ & USGS $W-2$ \\
\hline 80.00509 & B & $80 / 03 / 23$ & NBS 1566 Oyster Tissue \\
\hline 80.00510 & $w$ & $80 / 01 / 01$ & NBS $4926 \mathrm{C}$ Tritium in Water \\
\hline 80.00511 & $w$ & $80 / 01 / 01$ & NBS $4926 \mathrm{C}$ Tritium in Water \\
\hline 80.00512 & $w$ & $80 / 01 / 01$ & NBS $4926 C$ Tritium in Water \\
\hline 80.00513 & $W$ & $80 / 01 / 01$ & NBS 4926C Tritium in Water \\
\hline 80.00514 & w & $80 / 01 / 01$ & NBS $4926 \mathrm{C}$ Tritium in Water \\
\hline 80.00515 & $S$ & $78 / 02 / 15$ & CCRMP SY2 Rock \\
\hline 80.00516 & $\mathbf{S}$ & $78 / 02 / 15$ & CCRMP SY3 Rock \\
\hline 80.00517 & $\mathbf{S}$ & $78 / 02 / 15$ & CCRMP MRGI Rock \\
\hline 80.00518 & S & $78 / 02 / 15$ & CCRMP BLI Ore \\
\hline 80.00519 & $\mathrm{~S}$ & $78 / 02 / 15$ & CCRMP BL2 Ore \\
\hline 80.00520 & S & $78 / 02 / 15$ & CCRMP BL3 Ore \\
\hline 80.00521 & $S$ & $78 / 02 / 15$ & CCRMP BLA Ore \\
\hline 80.00522 & $S$ & $78 / 02 / 15$ & CCRMP DH1 Ore \\
\hline 80.00523 & $\mathbf{S}$ & $78 / 02 / 15$ & CCRMP DL1 Ore \\
\hline 80.00524 & $\mathrm{~S}$ & $78 / 02 / 15$ & IAEA S2 Uranium Ore \\
\hline 80.00525 & $\mathbf{S}$ & $78 / 02 / 15$ & IAEA S4 Uranium Ore \\
\hline 80.00526 & $\mathbf{S}$ & $78 / 02 / 15$ & JDEN 467 Uranium Ore \\
\hline 80.00527 & $\mathbf{S}$ & $78 / 02 / 15$ & JDEN 25 Uranium Ore \\
\hline 80.00528 & $\mathbf{S}$ & $78 / 02 / 15$ & JDEN 33 Uranium Ore \\
\hline 80.00529 & $\mathbf{S}$ & $78 / 02 / 15$ & JDEN 463 Uranium Ore \\
\hline 80.00530 & $\mathbf{S}$ & $78 / 02 / 15$ & IAEA S3 Uranium Ore \\
\hline 80.00531 & V & $78 / 02 / 15$ & NBS 1570 Spinach \\
\hline 80.00532 & $\mathrm{~V}$ & $78 / 02 / 15$ & NBS 1571 Orchard Leaves \\
\hline 80.00533 & $\mathrm{v}$ & $78 / 02 / 15$ & NBS 1573 Tomato Leaves \\
\hline 80.00534 & $\mathrm{v}$ & $78 / 02 / 15$ & NBS 1575 Pine Needles \\
\hline 80.00535 & B & $78,02 / 15$ & NBS 1577 Bovine Liver \\
\hline 80.00536 & $\mathbf{S}$ & $78 / 02 / 15$ & NBS $1632 \mathrm{Coal}$ \\
\hline 80.00537 & $\mathbf{S}$ & $78 / 02 / 15$ & NBS 1633 Fly Ash \\
\hline 80.00538 & W & $78 / 02 / 15$ & NBS $1641 \mathrm{Hg}$ in Water \\
\hline 80.00539 & $\mathbf{w}$ & $78 / 02 / 15$ & NBS $1642 \mathrm{Hg}$ in Water \\
\hline 80.00540 & $w$ & $78 / 02 / 15$ & NBS $1642 \mathrm{~A} \mathrm{Hg}$ in Water \\
\hline
\end{tabular}


TABLE I (cont)

\begin{tabular}{|c|c|c|c|}
\hline $\begin{array}{c}\text { Sample } \\
\text { Number } \\
\end{array}$ & $\begin{array}{c}\text { Sample } \\
\text { Type } \\
\end{array}$ & $\begin{array}{c}\text { Date } \\
\text { Received }\end{array}$ & $\begin{array}{l}\text { Sample } \\
\text { Name }\end{array}$ \\
\hline 80.00541 & $\mathbf{M}$ & $79 / 02 / 15$ & NBS $950 \mathrm{~A} \quad U_{3} O_{4}$ \\
\hline 80.00542 & $\mathbf{M}$ & $78 /(12 / 15$ & NBS $83 \mathrm{C} \mathrm{As}_{2} \mathrm{O}_{3}$ \\
\hline 80.00543 & $\mathbf{M}$ & $78 / 02 / 15$ & NBS $193 \mathrm{KNO}_{3}$ \\
\hline 80.00544 & $\mathbf{M}$ & 78/02ĩis & NBS $194 \mathrm{NH}_{4} \mathrm{H}_{2} \mathrm{PO}_{4}$ \\
\hline 80.00545 & $\mathbf{w}$ & $78 / 02 / 15$ & NBS $2671 \mathrm{~F}$ in Urine-Low Level \\
\hline 80.00546 & $\mathbf{S}$ & $78 / 02 / 15$ & NBS 79A Fluorspar \\
\hline 80.00547 & $\mathbf{S}$ & $78 / 02 / 15$ & NBS 120B Phosphate Rock \\
\hline 80.00548 & $\mathbf{S}$ & $78 / 02 / 15$ & NBS 91 Opal Glass \\
\hline 80.00549 & $\mathbf{S}$ & $78 / 02 / 15$ & NBS 633 Red Cap Cement \\
\hline 80.00550 & $\mathbf{S}$ & $78 / 02 / 15$ & NBS 635 Blue Cap Cement \\
\hline 80.00551 & $\mathbf{M}$ & $78 / 02 / 15$ & NBS 610 Glass \\
\hline 80.00552 & $\mathbf{M}$ & $78 / 02 / 15$ & NBS 113A Zinc Concentrate \\
\hline 80.00553 & $\mathbf{M}$ & $78 / 02 / 15$ & NBS 53E Pb \\
\hline 80.00554 & $\mathbf{M}$ & $78 / 02 / 15$ & NBS 348 Steel \\
\hline 80.00555 & $\mathbf{M}$ & $78 / 02 / 15$ & NBS 86C Al \\
\hline 80.00556 & $\mathbf{M}$ & $78 / 02 / 15$ & NBS 133A Sted \\
\hline 80.00557 & $\mathbf{M}$ & $78 / 02 / 15$ & NBS 168 Co-Ni-Cr-Alloy \\
\hline 80.00558 & $\mathbf{M}$ & $78 / 02 / 15$ & NBS $629 \mathrm{Zn}$ Alloy \\
\hline 80.00559 & $\mathbf{M}$ & $78 / 02 / 15$ & NBS 1197 Alloy \\
\hline 80.00561 & $\mathbf{M}$ & $78 / 02 / 15$ & NBS 1091 Steel \\
\hline 80.00562 & $\mathbf{M}$ & $78 / 02 / 15$ & NBS 1093 Steel \\
\hline 80.00563 & $\mathbf{M}$ & $78 / 02 / 15$ & NBS 644 Steel \\
\hline 80.00564 & $\mathbf{M}$ & $78 / 02 / 15$ & NBS 1135 Steel \\
\hline 80.00565 & $\mathbf{M}$ & $78 / 02 / 15$ & NBS 1262 Steel \\
\hline 80.00566 & $\mathbf{M}$ & $78 / 02 / 15$ & NBS 1264 Steel \\
\hline 80.00567 & B & $78 / 02 / 15$ & Kodak TEG SOA \\
\hline 80.00568 & $\mathbf{B}$ & $78 / 02 / 15$ & Kodak TEG 50B \\
\hline 80.00569 & $\mathbf{B}$ & $78 / 02 / 15$ & Kodak TEG 50C \\
\hline 80.00570 & $\mathbf{S}$ & $78 / 02 / 15$ & USGS AVG-1 \\
\hline 80.00571 & $\mathbf{S}$ & $78 / 02 / 15$ & USGS G-2 \\
\hline 80.00572 & $\mathbf{S}$ & $78 / 02 / 15$ & USGS BCR-1 \\
\hline 80.00573 & $\mathbf{S}$ & $78 / 02 / 15$ & USGS PCC-1 \\
\hline 80.00574 & $\mathbf{S}$ & $78 / 02 / 15$ & USGS GSP-1 \\
\hline 80.00575 & $\mathbf{S}$ & $78 / 02 / 15$ & USGS GXR-1 \\
\hline 80.00577 & $\mathbf{S}$ & $78 / 02 / 15$ & USGS GXR-2 \\
\hline 80.00579 & $\mathbf{S}$ & $78 / 02 / 15$ & USGS GXR-3 \\
\hline 80.00581 & $\mathbf{S}$ & $78 / 02 / 15$ & USGS GXR-4 \\
\hline 80.00583 & $\mathbf{S}$ & $78 / 02 / 15$ & USGS GXR-5 \\
\hline 80.00585 & $\mathbf{S}$ & $78 / 02 / 15$ & USGS GXR-6 \\
\hline 80.00587 & $\mathbf{S}$ & $78 / 02 / 15$ & CCRMP BL5 Ore \\
\hline 80.00588 & $\mathbf{w}$ & $78 / 01 / 01$ & NBS $4958{ }^{226} \mathrm{Ra}$ in Water \\
\hline 80.00589 & $\mathbf{w}$ & $78 / 02 / 15$ & EPA No. 1 Trace Metals in Water \\
\hline 80.00590 & $\mathbf{w}$ & $78 / 02 / 15$ & EPA No. 2 Trace Metals in Water \\
\hline
\end{tabular}


TABLE I (con)

\begin{tabular}{|c|c|c|c|}
\hline $\begin{array}{l}\text { Semple } \\
\text { Nomber }\end{array}$ & $\begin{array}{l}\text { Sumple } \\
\text { Type }\end{array}$ & $\begin{array}{c}\text { Date } \\
\text { Recoived }\end{array}$ & $\begin{array}{l}\text { Senple } \\
\text { Neme }\end{array}$ \\
\hline 80.00591 & $\mathbf{W}$ & $78 / 02 / 15$ & EPA No. 3 Trace Metala in Water \\
\hline 80.00592 & $\mathbf{W}$ & $78 / 05 / 15$ & EPA No. 4 Mineral Analyses in Water \\
\hline 80.00593 & $\mathbf{w}$ & $78 / 05 / 15$ & EPA No. 5 Mineral Analyees in Water \\
\hline 80.00594 & $\mathbf{S}$ & $78 / 07 / 17$ & CCRMP Regosolic Soil SOI \\
\hline 80.00596 & $\mathbf{S}$ & $78 / 07 / 17$ & CCRMP Podzolic Soil SO2 \\
\hline 80.00598 & $\mathbf{s}$ & $78 / 07 / 17$ & CCRMP Calcereous C Horizon Soil SO3 \\
\hline 80.00600 & $\mathbf{s}$ & $78 / 07 / 17$ & CCRMP Chernozemic A Horizon Soil SO4 \\
\hline 80.00602 & $\mathbf{w}$ & $78 / 10 / 12$ & H8 $U$ in Water \\
\hline 80.00603 & $\mathbf{w}$ & $78 / 08 / 15$ & H8 TDS \\
\hline 80.00604 & $\mathbf{W}$ & $79 / 02 / 03$ & $H 8 \mathrm{U}$ in Water \\
\hline 80.00605 & $\mathbf{S}$ & $79 / 01 / 15$ & NBS 1632A Bituminous Coal \\
\hline 80.00606 & $\mathbf{s}$ & $79 / 02 / 15$ & NBS 1635 Subbituminous Coal \\
\hline 80.00607 & $\mathbf{A}$ & $79 / 03 / 01$ & NBS 1648 Air Particulate \\
\hline 80.00608 & $\mathbf{s}$ & $79 / 03 / 01$ & NBS 1645 River Sediment \\
\hline 80.00609 & $\mathbf{s}$ & $79 / 03 / 01$ & CCRMP DL-1a Ore \\
\hline 80.00610 & $\mathbf{s}$ & $79 / 05 / 01$ & USGS BHVO-1 \\
\hline 80.00611 & $\mathbf{s}$ & $79 / 05 / 01$ & USGS MAG-1 \\
\hline 80.00612 & $\mathbf{s}$ & $79 / 05 / 01$ & USGS QLO-1 \\
\hline 80.00613 & $\mathbf{s}$ & $79 / 05 / 01$ & USGS RGM-1 \\
\hline 80.00614 & $\mathbf{S}$ & $79 / 05 / 01$ & USGS SCo-1 \\
\hline 80.00615 & $\mathbf{S}$ & $79 / 03 / 01$ & USGS SDC-1 \\
\hline 80.00616 & $\mathbf{s}$ & $799 / 05 / 01$ & USGS SGR-1 \\
\hline 80.00617 & $\mathbf{s}$ & $79 / 05 / 01$ & USGS STM-1 \\
\hline 80.00618 & $\mathbf{w}$ & $78 / 02 / 01$ & NBS $2671 \mathrm{~F}$ in Urine-High Level \\
\hline 80.00619 & $\mathbf{W}$ & $78 / 10 / 01$ & H8 $\mathrm{U}$ in Water \\
\hline 80.00620 & $\mathbf{w}$ & $78 / 01 / 01$ & NBS $4921 \mathrm{C}^{22} \mathrm{Na}$ in Water \\
\hline 80.00621 & $\mathbf{W}$ & $78 / 01 / 01$ & NBS $4921 \mathrm{C}^{22} \mathrm{Na}$ in Water \\
\hline 80.00622 & $\mathbf{W}$ & $78 / 01 / 01$ & H8 Deep Well (PM-1) Water Blank \\
\hline 80.00623 & $\mathbf{S}$ & $78 / 01 / 01$ & NBS $4921 \mathrm{C}^{22} \mathrm{Na}$ on Soil GTP-3 \\
\hline 80.00624 & $\mathbf{S}$ & $78 / 01 / 01$ & NBS $4921 C^{22} \mathrm{Na}$ on Soil GTP-3 \\
\hline 80.00625 & $\mathbf{s}$ & $78 / 01 / 01$ & NBS $4921 \mathrm{C}^{22} \mathrm{Na}$ on Soil GTP-3 \\
\hline 80.00626 & $\mathbf{s}$ & $78 / 01 / 01$ & NBS $4921 \mathrm{C}^{22} \mathrm{Ne}$ on Soil GTP-3 \\
\hline 80.00627 & $\mathbf{s}$ & $78 / 01 / 01$ & NRS $4921 C^{22}$ Na on Chamita Sediment \\
\hline 80.00628 & $\mathbf{s}$ & $78 / 01 / 01$ & NES $4921 C^{22}$ Na on Chamita Sediment \\
\hline 80.00629 & $\mathbf{s}$ & $78 / 01 / 01$ & NBS $4921 C^{22} \mathrm{Na}$ on Chamita Sediment \\
\hline 80.00630 & B & $79 / 07 / 01$ & NBS 1567 Wheat Flour \\
\hline 80.00631 & B & $79 / 07 / 01$ & NBS 1568 Rice Flour \\
\hline 80.00632 & $\mathbf{V}$ & $79 / 07 / 01$ & NBS 1569 Brewer's Yeast \\
\hline 80.00633 & $\mathbf{s}$ & $79 / 07 / 01$ & NBS 1633A Coal Fly Ash \\
\hline 80.00634 & $\mathbf{s}$ & $78 / 02 / 15$ & NBS $1631 \mathrm{~A} S$ in Conl \\
\hline 80.00635 & $\mathbf{S}$ & $78 / 02 / 15$ & NBS $1631 B \mathrm{~S}$ in Conl \\
\hline 80.00636 & $\mathbf{S}$ & $78 / 02 / 15$ & NBS $1631 \mathrm{~S}$ in Conl \\
\hline 80.00637 & $\mathbf{w}$ & $80 / 04 / 01$ & EML ${ }^{137} \mathrm{Cs}$ \\
\hline
\end{tabular}


TABLE 1 (cont)

\begin{tabular}{|c|c|c|c|}
\hline $\begin{array}{c}\text { Sample } \\
\text { Number } \\
\end{array}$ & $\begin{array}{c}\text { Sample } \\
\text { Type } \\
\end{array}$ & $\begin{array}{c}\text { Date } \\
\text { Received }\end{array}$ & $\begin{array}{c}\text { Sample } \\
\text { Name }\end{array}$ \\
\hline 80.00638 & $w$ & $80 / 04 / 01$ & $\mathrm{EML}{ }^{137} \mathrm{Cs}$ \\
\hline 80.00639 & $w$ & $80 / 04 / 01$ & EML ${ }^{137} \mathrm{Cs}$ \\
\hline 80.00640 & $\mathbf{S}$ & $80 / 04 / 01$ & EML ${ }^{137} \mathrm{Cs}$ \\
\hline 80.00641 & $\mathrm{~S}$ & $80 / 04 / 01$ & $\mathrm{EML}{ }^{137} \mathrm{Cs}$ \\
\hline 80.00642 & $\mathrm{~S}$ & $80 / 04 / 01$ & EML ${ }^{137} \mathrm{Cs}$ \\
\hline 80.00643 & $w$ & $80 / 04 / 25$ & EPA Trace Metals-1 \\
\hline 80.00644 & $w$ & $80 / 04 / 25$ & EPA Trace "etals-2 \\
\hline 80.00645 & W & $80 / 04 / 25$ & EPA Nutrieıts- 1 \\
\hline 80.00646 & $w$ & $80 / 04 / 25$ & EPA Nutrients-2 \\
\hline 80.00647 & $w$ & $80 / 04 / 25$ & EPA Nutrients-5 \\
\hline 80.00648 & $w$ & $80 / 04 / 25$ & EPA Nutrients- 6 \\
\hline 80.00649 & $W$ & $80 / 04 / 25$ & EPA Trace Metals- 1 \\
\hline 80.00650 & $w$ & $80 / 04 / 25$ & EPA Trace Metals-2 \\
\hline 80.00651 & W & $80 / 04 / 25$ & EPA Trace Metals 3 \\
\hline 80.00552 & $w$ & $80 / 04 / 25$ & EPA Mineral Analyses-1 \\
\hline 80.00653 & w & $80 / 04 / 25$ & EPA Mineral Analyses-2 \\
\hline 80.00654 & $w$ & $80 / 04 / 25$ & EPA Trace Metals-1 \\
\hline 80.00655 & $w$ & $80 / 04 / 25$ & EPA Trace Metals-10 \\
\hline 80.00656 & $W$ & $80 / 11 / 11$ & $\mathrm{H} 8 \mathrm{U}$ in $W$ ater \\
\hline 80.00657 & $\mathrm{~S}$ & $80 / 10 / 01$ & CCRMP DH IA Ore \\
\hline 80.00560 & A & $80 / 05 / 20$ & EPA As Filter Strips-0 \\
\hline 80.00661 & A & $80 / 05 / 20$ & EPA As Filter Strips--1 \\
\hline 80.00662 & A & $80 / 05 / 20$ & EPA As Filter Strips -2 \\
\hline 90.00663 & A & $80 / 05 / 20$ & EPA As Filter Strips-3 \\
\hline 80.00664 & A & $80 / 05 / 20$ & EPA As Filter Strips -4 \\
\hline 80.00665 & A & $80 / 05 / 20$ & EPA As Filter Strips- -5 \\
\hline 80.00666 & A & $80 / 05 / 20$ & EPA As Filter Strips -6 \\
\hline 80.00667 & A & $80 / 05 / 20$ & EPA As Filter Strips- - \\
\hline 80.00668 & A & $80 / 05 / 20$ & EPA As Filter Strips -8 \\
\hline 80.00669 & A & $80 / 05 / 20$ & EPA As Filter Strips-9 \\
\hline 80.00670 & A & $80 / 05 / 20$ & EPA $\mathrm{SO}_{4}-\mathrm{NO}_{3}$ Filter Strips -0 \\
\hline 80.00671 & A & $80 / 05 / 20$ & EPA $\mathrm{SO}_{4}-\mathrm{NO}_{3}$ Filter Strips-1 \\
\hline 80.00672 & A & $80 / 05 / 20$ & EPA $\mathrm{SO}_{4}-\mathrm{NO}_{3}$ Filter Strips -2 \\
\hline 80.00673 & A & $80 / 05 / 20$ & EPA $\mathrm{SO}_{4}-\mathrm{NO}_{3}$ Filter Strips-3 \\
\hline 80.00674 & A & $80 / 05 / 20$ & EPA $\mathrm{SO}_{4}-\mathrm{NO}_{3}$ Filter Strips-4 \\
\hline 80.00675 & A & $80 / 05 / 20$ & EPA $\mathrm{SO}_{4}-\mathrm{NO}_{3}$ Filter Strips-5 \\
\hline 80.00676 & A & $80 / 05 / 20$ & EPA $\mathrm{SO}_{4}-\mathrm{NO}_{3}$ Filter Strips-6 \\
\hline 80.00677 & A & $80 / 05 / 20$ & EPA $\mathrm{SO}_{4}-\mathrm{NO}_{3}$ Filter Strips -7 \\
\hline 80.00678 & A & $80 / 05 / 20$ & EPA $\mathrm{SO}_{4}-\mathrm{NO}_{3}$ Filter Strips-8 \\
\hline 80.00679 & A & $80 / 05 / 20$ & EPA $\mathrm{SO}_{4}-\mathrm{NO}_{3}$ Filter Strips-9 \\
\hline 80.00680 & A & $80 / 05 / 20$ & EPA Pb Filter Strip-0 \\
\hline 80.00681 & A & $80 / 05 / 20$ & EPA Pb Filter Strip-I \\
\hline 80.00682 & $A$ & $80 / 05 / 20$ & EPA $\mathrm{Pb}$ Filter Strip-2 \\
\hline
\end{tabular}


TABLE I (cont)

\begin{tabular}{|c|c|c|c|}
\hline $\begin{array}{c}\text { Sample } \\
\text { Number } \\
\end{array}$ & $\begin{array}{c}\text { Sample } \\
\text { Type } \\
\end{array}$ & $\begin{array}{c}\text { Date } \\
\text { Received }\end{array}$ & $\begin{array}{c}\text { Sample } \\
\text { Name }\end{array}$ \\
\hline 80.00683 & A & $80 / 05 / 20$ & EPA Pb Filter Strip - 3 \\
\hline 80.00584 & A & $80 / 05 / 20$ & EPA Pb Filter Strip-4 \\
\hline 80.00685 & $A$ & $80 / 05 / 20$ & EPA Pu Filter Strip -5 \\
\hline 80.00686 & $\therefore$ & $80 \cdot 05 / 20$ & EPA Pb Filter Strip- \\
\hline 80.00687 & $\therefore$ & $80 / 05 / 20$ & EPA Pb Filter Strip- 7 \\
\hline 80.00688 & $\therefore$ & $80 / 05 / 20$ & EPA Pb Filter Strip- 8 \\
\hline $80.00+89$ & $A$ & $80: 05 / 20$ & EPA Pb Filter Stilp- 9 \\
\hline 80.00690 & $S$ & $80.05 / 22$ & CRPG Feldspath FK $\cdot \mathrm{N}$ \\
\hline 80.00691 & S & $80 / 05,22$ & CRPG Granite CH \\
\hline 80.00692 & $S$ & $80 / 05 / 22$ & CRPG Verre Synthetque VS $N$ \\
\hline 80.00643 & s & $80 / 05 / 22$ & CRPG Granite GA \\
\hline 80.00644 & $S$ & $80 / 05 / 22$ & CFPG Anorihosite AN-CB \\
\hline 80.00695 & $\$$ & $80 / 05 / 22$ & CRPG Disthene DT-IV \\
\hline 80.00640 & $\varsigma$ & $80: 05,22$ & CRPG Basalte BR \\
\hline 80.00697 & $\mathrm{~S}$ & $80 / 05 / 22$ & CRPG Mica-Mg \\
\hline 80.00598 & $s$ & $80,05,22$ & CRPG Gliuconite GL O (grains) \\
\hline 80.00699 & $s$ & $80 / 05 / 22$ & CRPG Granite MA-N \\
\hline 80.00700 & $s$ & $80 / 05 / 22$ & CRPG Bauxite BX-N \\
\hline 80.00701 & $S$ & 80,0522 & CRPG Diorite DR $-N$ \\
\hline 80.00702 & $S$ & $80 / 05,22$ & CRPG Serpentine ¿B $\mathrm{N}$ \\
\hline 80.00703 & $S$ & $80 / 05 / 22$ & CRPG Mica--Fe \\
\hline 80.00704 & $\$$ & $80 \cdot 05,22$ & CRPG Basalte BE- N \\
\hline 80.00705 & $S$ & $80: 05 / 22$ & CRPG Glauconite GL O (ponder) \\
\hline 85.00706 & 5 & $80: 05 / 22$ & CRPG Grante GS-N \\
\hline
\end{tabular}

Stable Element Quality Assurance

To evaluate the quality assurance data for the year, one can generate quality control charts and/or the data can be summarized as a mean $\overline{(x)} \pm$ one standard deviation(s) of the ratio of the $\mathrm{H} .8$ value to the cerified or consensus value ( $\mathrm{CV}$ ). Since the $\mathrm{H}-8$ laboratory operates on a denand basis, most analyses are performed on an irregular, infrequent basis. The scheme of control charts does not address this mode of operation well. Results of the mean of the $\mathrm{H}-8 / \mathrm{CV}$ ratio approach arc shown in Iable II, and control charts are presented in Appendix B. Silicate QA materials deposited on blank (unexposed) air filters were used as the air particulate QA this year and are combined under the silicate category in Table II. All QA raw data are given in Appendix C. Table III con- tains the summary of elemental and isotopic analyses completed by $\mathrm{H}-8$ as a function of matrix type, and Table IV gives this summary as a function of analytical technique employed. The abbreviations used in Table IV are defined in Fig. 1. The symbols in Fig. 1 are used in the control charts presented in Appendix $\mathbf{B}$.

Examination of the data in Table II reveals that only $\mathrm{Lu}$ in silicates and hardness in water are grossly out of control (beyond the $\pm 20 \%$ limits set in Ref. 1 for data rejection). The single standard deviations for the rest of the analyses overlap the 1.00 ratio value, indicating overall control. The relative size of the standard deviation of the ratios in Table II gives a good indication of how good our overall agreement is with the certified or consensus values. The following percentages of entries in Table II fall within given ranges. 
TABLE II

SUMMARY OF H-8/CV RATIO FOR STABLE ELEMENT AND SELECTED RADIOCHEMICAL ANALYSES BY MATRIX

\begin{tabular}{|c|c|c|c|c|c|c|}
\hline \multirow[b]{2}{*}{ Analysis } & \multicolumn{2}{|c|}{ Silicate } & \multicolumn{2}{|c|}{ Water } & \multicolumn{2}{|c|}{ Biological } \\
\hline & $\bar{x} \pm s$ & $\begin{array}{c}\text { No. } \\
\text { Reports }\end{array}$ & $\bar{x} \pm s$ & $\begin{array}{c}\text { No. } \\
\text { Reports }\end{array}$ & $\bar{x} \pm s$ & $\begin{array}{c}\text { No. } \\
\text { Reports } \\
\end{array}$ \\
\hline Ag & $1.00 \pm 0.05$ & 12 & $0.88 \pm 0.18$ & 4 & & \\
\hline Al & $0.99 \pm 0.04$ & 46 & & & & \\
\hline As & $1.01 \pm 0.13$ & 42 & $1.00 \pm 0.11$ & 73 & & \\
\hline B & $1.00 \pm 0.10$ & 27 & & & $1.03 \pm 0.08$ & 13 \\
\hline $\mathbf{B a}$ & $1.07 \pm 0.12$ & 58 & $1.17 \pm 0.11$ & 5 & & \\
\hline Be & & & $1.00 \pm 0.05$ & 18 & & \\
\hline $\mathbf{B r}$ & $0.90 \pm 0.14$ & 5 & & & & \\
\hline $\mathrm{Ca}$ & $0.96 \pm 0.14$ & 3 & $1.09 \pm 0.20$ & 12 & $0.85 \pm 0.19$ & 3 \\
\hline Cd & $0.90 \pm 0.39$ & 8 & $0.97 \pm 0.20$ & 90 & & \\
\hline $\mathrm{Ce}$ & $0.99 \pm 0.06$ & 57 & & & & \\
\hline $\mathrm{Cl}$ & $1.10 \pm 0.11$ & 14 & $1.02 \pm 0.07$ & 56 & $0.99 \pm 0.08$ & 8 \\
\hline Co & $1.01 \pm 0.07$ & 84 & 1.00 & 1 & & \\
\hline Conductivity & & & $0.99 \pm 0.08$ & 5 & & \\
\hline $\mathrm{Cr}$ & $1.00 \pm 0.09$ & 79 & $1.02 \pm 0.18$ & 24 & & \\
\hline Cs & $1.02 \pm 0.14$ & 49 & & & & \\
\hline${ }^{137} \mathrm{Cs}$ & $1.13 \pm 0.12$ & 9 & $1.00 \pm 0.06$ & 19 & & \\
\hline $\mathrm{Cu}$ & & & $1.03 \pm 0.09$ & 5 & & \\
\hline Dy & $1.07 \pm 0.17$ & 6 & & & & \\
\hline $\mathbf{E u}$ & $0.97 \pm 0.08$ & 59 & & & & \\
\hline $\mathbf{F}$ & & & $1.03 \pm 0.32$ & 49 & & \\
\hline Fe & $0.99 \pm 0.05$ & 94 & & & & \\
\hline $\mathrm{Ga}$ & $0.96 \pm 0.11$ & 9 & & & & \\
\hline Hardness & & & $1.75 \pm 0.88$ & 6 & & \\
\hline Hf & $0.98 \pm 0.12$ & 46 & & & & \\
\hline $\begin{array}{l}\mathbf{H g} \\
{ }^{3} \mathbf{H}\end{array}$ & $0.93 \pm 0.10$ & 9 & $\begin{array}{l}0.98 \pm 0.05 \\
1.16 \pm 0.19\end{array}$ & $\begin{array}{r}4 \\
100\end{array}$ & $1.06 \pm 0.04$ & 3 \\
\hline $\mathbf{K}$ & $1.02 \pm 0.10$ & 26 & $1.03 \pm 0.20$ & 12 & $0.98 \pm 0.13$ & 4 \\
\hline La & $1.06 \pm 0.13$ & 53 & & & & \\
\hline $\mathbf{L i}$ & $0.91 \pm 0.28$ & 8 & & & & \\
\hline $\mathbf{L u}$ & 1.64 & 2 & & & & \\
\hline $\mathbf{M g}$ & & & $1.03 \pm 0.25$ & 12 & & \\
\hline $\mathbf{M n}$ & $1.07 \pm 0.19$ & 43 & 1.03 & 2 & $0.99 \pm 0.04$ & 6 \\
\hline Mo & $1.08 \pm 0.08$ & 3 & 1.16 & 1 & & \\
\hline $\mathbf{N a}$ & $1.01 \pm 0.09$ & 54 & $1.04 \pm 0.06$ & 10 & $1.06 \pm 0.07$ & 4 \\
\hline${ }^{22} \mathrm{Na}$ & $1.06 \pm 0.10$ & 12 & $1.15 \pm 0.08$ & 19 & & \\
\hline Nd & 0.94 & 2 & & & & \\
\hline $\mathbf{N i}$ & $0.86 \pm 0.02$ & 3 & 1.08 & 2 & & \\
\hline $\mathrm{NO}_{3}$ & & & $1.00 \pm 0.01$ & 3 & & \\
\hline $\begin{array}{l}\mathrm{Pb} \\
216 \mathrm{p}_{0}\end{array}$ & 07 & 10 & $0.92 \pm 0.25$ & 12 & & \\
\hline
\end{tabular}


TABLE II (cont)

\begin{tabular}{|c|c|c|c|c|c|c|}
\hline \multirow[b]{2}{*}{ Antyais } & \multicolumn{2}{|c|}{ Silicate } & \multicolumn{2}{|c|}{ Water } & \multicolumn{2}{|c|}{ Biological } \\
\hline & $\bar{x} \pm=$ & $\begin{array}{c}\text { No. } \\
\text { Reports }\end{array}$ & $\bar{x} \pm s$ & $\begin{array}{c}\text { No. } \\
\text { Reports }\end{array}$ & $\bar{x} \pm 8$ & $\begin{array}{c}\text { No. } \\
\text { Reports }\end{array}$ \\
\hline $\mathbf{R} \mathbf{b}$ & $1.02 \pm 0.10$ & 72 & & & & \\
\hline $\mathbf{S b}$ & $1.05 \pm 0.05$ & 25 & & & & \\
\hline Sc & $1.01 \pm 0.08$ & 52 & & & & \\
\hline Se & $0.97 \pm 0.09$ & 24 & $1.12 \pm 0.11$ & 10 & & \\
\hline Sm & $1.01 \pm 0.13$ & 49 & & & & \\
\hline So. & & & $0.99 \pm 0.10$ & 29 & & \\
\hline Sr & $0.92 \pm 0.06$ & 11 & & & & \\
\hline $\mathbf{T a}$ & $0.86 \pm 0.15$ & 45 & & & & \\
\hline $\mathbf{T b}$ & $1.02 \pm 0.17$ & 9 & & & & \\
\hline TDS & & & $0.99 \pm 0.04$ & 6 & & \\
\hline Th & $1.01 \pm 0.05$ & 102 & & & & \\
\hline $\mathbf{T i}$ & $1.01 \pm 0.06$ & 14 & & & & \\
\hline $\mathbf{U}$ & $1.00 \pm 0.08$ & 248 & $0.98 \pm 0.04$ & 45 & $1.02 \pm 0.09$ & 6 \\
\hline $\mathbf{V}$ & $1.09 \pm 0.21$ & 40 & 1.02 & 1 & & \\
\hline $\mathbf{W}$ & $0.98 \pm 0.11$ & 11 & & & & \\
\hline $\mathbf{Y b}$ & $1.00 \pm 0.22$ & 19 & & & & \\
\hline $\mathbf{Z n}$ & $1.02 \pm 0.23$ & 6 & $1.06 \pm 0.16$ & 6 & & \\
\hline $\mathbf{Z r}$ & $0.95 \pm 0.02$ & 3 & & & & \\
\hline
\end{tabular}

No $s$ (insufficient data points)

$s \leq 0.10$

$8 \%$

$49 \%$

$33 \%$

$0.11 \leq \mathrm{s} \leq 0.20$

$10 \%$

Compared with the previous year's results, there has been a sharp decline in the first and fourth categories above, with about equal increase in the middle categories. This indicates improvement in our overall QA efforts over the previous year.

However, a number of analyses need attention as indicated by the large single standard deviation in Table II. Specifically, the control of $\mathrm{Ag}, \mathrm{Ba}, \mathrm{Ca}, \mathrm{Cd}, \mathrm{F}, \mathrm{K},{ }^{3} \mathrm{H}$, ${ }^{22} \mathrm{Na}, \mathrm{Pb}$, and $\mathrm{Se}$ in water; $\mathrm{Br}, \mathrm{Ca}, \mathrm{Cd}, \mathrm{Li}, \mathrm{Ni}, \mathrm{Ta}, \mathrm{V}, \mathrm{Yb}$, and $\mathrm{Zn}$ in silicates; and $\mathrm{Ca}$ in biologicals needs improvement.

The QA program of EML has been expanded to include selected stable elements in various matrices. The results of our participation in this program are given in Appendix D and have not been included in Table II since the certified value is established by EML only, and not by either a recognized national standards laboratory or by interlaboratory consensus.

\section{Radiochemical Quality Assurance}

Our laboratory continues to participate in both the EML and EPA Class II secondary QA standards programs. The results of these analyses are given in Appendices $D$ and $E$, respectively. We have developed several of our own internal Class 11 radioactive materials employing NBS, EPA, and EML "certified" radionuclide solution standards. Most of the EPA solutions were prepared and standardized by NBS. In the cases of ${ }^{22} \mathrm{Na}$ and ${ }^{137} \mathrm{Cs}$, we have prepared doped soil samples as well. The summary of these results is included in Table II and the individual data are given in Appendix C. A summary oi the radiochemical determinations completed as a function of matrix is included in Table III.

\section{ACKNOWLEDGMENTS}

We thank Kathy Derouin, Verna Halloran, and Mary Lou Keigher for their assistance in the preparation of this oocument. We also thank the analysts vho, in addition to the authors, provided the data compiled in this 
TABLE III

SUMMARY OF ANALYTICAL. DETERMINATIONS COMPLETED DURING CY 1980 BY MATRIX

\begin{tabular}{|c|c|c|c|c|c|}
\hline Analys:s & Silicate & Water & Biological & Air & Total \\
\hline$\alpha-\beta$ & 179 & 476 & 20 & 371 & 1046 \\
\hline$\gamma$ & 328 & 366 & 74 & 39 & 807 \\
\hline${ }^{3} \mathrm{H}$ & 225 & 975 & 30 & 194 & 1424 \\
\hline${ }^{7} \mathrm{Be}$ & 40 & 76 & & 9 & 125 \\
\hline${ }^{22} \mathrm{Na}$ & 40 & 76 & 9 & & 125 \\
\hline${ }^{40} \mathrm{~K}$ & 2 & & 3 & & 5 \\
\hline${ }^{31} \mathrm{Cr}$ & & 9 & & & 9 \\
\hline${ }^{60} \mathrm{Co}$ & 2 & 18 & & & 20 \\
\hline${ }^{65} \mathrm{Zn}$ & & 12 & & & 12 \\
\hline${ }^{90} \mathrm{Sr}$ & 225 & 107 & 31 & 4 & 367 \\
\hline${ }^{106} \mathrm{Ru}$ & & 12 & & & 12 \\
\hline${ }^{131} \mathrm{I}$ & & & 17 & 3 & 20 \\
\hline${ }^{134} \mathrm{Cs}$ & & 18 & & & 18 \\
\hline${ }^{137} \mathrm{Cs}$ & 273 & 216 & 32 & 18 & 539 \\
\hline${ }^{140} \mathrm{Ba}$ & 17 & & & & 17 \\
\hline${ }^{226} \mathrm{Ra}$ & 69 & 7 & & & 76 \\
\hline${ }^{227} \mathrm{Ac}$ & 67 & & & & 67 \\
\hline${ }^{238} \mathrm{Pu},{ }^{239} \mathrm{Pu},{ }^{246} \mathrm{Pu}$ & 432 & 167 & 21 & 86 & 706 \\
\hline${ }^{241} \mathrm{Am}$ & 108 & & & 86 & 194 \\
\hline $\mathrm{Ag}$ & 69 & 38 & 110 & & 217 \\
\hline Al & 337 & 5 & 88 & 95 & 525 \\
\hline As & 292 & 346 & 18 & 55 & 711 \\
\hline $\mathrm{Au}$ & & 35 & & & 35 \\
\hline$B$ & 144 & 192 & 108 & & 444 \\
\hline $\mathrm{Ba}$ & 405 & 29 & & & 434 \\
\hline $\mathrm{Be}$ & & 1 & 70 & & 71 \\
\hline $\mathrm{Br}$ & 48 & 32 & 112 & 91 & 283 \\
\hline $\mathrm{Ca}$ & 201 & 150 & 44 & & 395 \\
\hline $\mathrm{Cd}$ & 153 & 215 & 88 & & 456 \\
\hline $\mathrm{Ce}$ & 261 & & & & $26 ́ 1$ \\
\hline $\mathrm{Cl}$ & 336 & 243 & 200 & 4 & 783 \\
\hline Co & 291 & 8 & & 95 & 394 \\
\hline $\mathrm{Cr}$ & 301 & 37 & 158 & 95 & 591 \\
\hline Cs & 243 & 9 & & & 252 \\
\hline $\mathrm{Cu}$ & 132 & 13 & & 4 & 149 \\
\hline Dy & 116 & & & & 116 \\
\hline $\mathrm{Eu}$ & 273 & & & & 273 \\
\hline$F$ & 98 & 308 & 88 & & 494 \\
\hline $\mathrm{Fe}$ & 339 & 12 & 88 & 95 & 534 \\
\hline $\mathrm{Ga}$ & 65 & 32 & & & 97 \\
\hline Hf & 243 & & & & 243 \\
\hline $\mathrm{Hg}$ & 100 & 34 & 29 & & 163 \\
\hline
\end{tabular}


TABLE III (cont)

\begin{tabular}{|c|c|c|c|c|c|}
\hline Analysis & Silicate & Water & Biological & Air & Total \\
\hline$K$ & 180 & 148 & 44 & 91 & 463 \\
\hline $\mathrm{La}$ & 162 & & & 91 & 253 \\
\hline $\mathbf{L i}$ & 130 & 301 & 18 & & 449 \\
\hline Lu & 65 & & & & 65 \\
\hline $\mathrm{Mg}$ & 102 & 148 & & 2 & 252 \\
\hline $\mathrm{Mn}$ & 349 & 11 & 112 & 97 & 569 \\
\hline Mo & 40 & 39 & & & 79 \\
\hline $\mathrm{Na}$ & 340 & 147 & 24 & 97 & 608 \\
\hline $\mathrm{Nd}$ & 9 & & & & 9 \\
\hline $\mathrm{Ni}$ & 51 & 3 & & & 54 \\
\hline $\mathrm{Pb}$ & 30 & 29 & & 2 & 61 \\
\hline $\mathrm{Pd}$ & 65 & & & & 65 \\
\hline $\mathrm{Pt}$ & 65 & & & & 65 \\
\hline $\mathrm{Rb}$ & 277 & 5 & 88 & 91 & 461 \\
\hline $\mathbf{R h}$ & 65 & & & & 65 \\
\hline $\mathrm{Ru}$ & 65 & & & & 65 \\
\hline $\mathrm{Sb}$ & 173 & 5 & & 4 & 182 \\
\hline $\mathrm{Sc}$ & 252 & 5 & & 4 & 261 \\
\hline $\mathrm{Se}$ & 158 & 11 & & & 169 \\
\hline Si & 81 & 125 & & & 206 \\
\hline $\mathrm{Sm}$ & 162 & & & 79 & 241 \\
\hline $\mathrm{Sn}$ & & 3 & & & 3 \\
\hline $\mathrm{Sr}$ & 74 & & & & 74 \\
\hline $\mathrm{Ta}$ & 214 & & & & 214 \\
\hline $\mathrm{Tb}$ & 74 & & & & 74 \\
\hline Th & 515 & 5 & & & 520 \\
\hline $\mathrm{Ti}$ & 198 & & & 44 & 242 \\
\hline $\mathrm{U}$ & 1285 & 383 & 93 & 122 & 1883 \\
\hline${ }^{235} \mathrm{U}$ & 69 & & & & 69 \\
\hline${ }^{238} \mathrm{U}$ & 69 & & & & 69 \\
\hline $\mathrm{V}$ & 336 & 11 & & 91 & 438 \\
\hline W & 107 & 32 & & & 139 \\
\hline $\mathrm{Yb}$ & 87 & & & & 87 \\
\hline $\mathrm{Zn}$ & 47 & 7 & & 99 & 153 \\
\hline $\mathrm{Zr}$ & 9 & & & & 9 \\
\hline $\mathrm{pH}$ & & 106 & & & 106 \\
\hline Water Content & 254 & & & & 254 \\
\hline Conductivity & & 152 & & & 152 \\
\hline TDS & & 155 & & & 155 \\
\hline $\mathrm{CO}_{3}$ & & 152 & & & 152 \\
\hline $\mathrm{HCO}_{3}$ & & 152 & & & 152 \\
\hline Hardness & & 152 & & & 152 \\
\hline $\mathrm{NO}_{3}$ & 40 & 168 & 88 & 2 & 298 \\
\hline $\mathrm{SO}_{4}$ & 40 & 178 & 88 & 2 & 308 \\
\hline $\mathrm{PO}_{4}$ & 40 & 158 & 88 & & 286 \\
\hline
\end{tabular}


TABLE IV

SUMMARY OF ANALYITCAL DETERMINATIONS FOR CY1980

BY TECHNIQUE FOR STABLE ELEMENT LABORATORY

\begin{tabular}{|c|c|c|c|c|c|c|c|c|}
\hline Analysis & ITNA & IENA & $\mathbf{A} \mathbf{A}$ & FAA & ICP & IC & Other" & Total \\
\hline $\mathbf{A g}$ & 170 & 9 & & 31 & 7 & & & 217 \\
\hline Al & 525 & & & & & & & 525 \\
\hline As & 110 & 117 & & 449 & 7 & & 28 & 711 \\
\hline $\mathrm{Au}$ & 1 & 32 & 2 & & & & & 35 \\
\hline B & & & 117 & & 122 & & 205 & 444 \\
\hline $\mathrm{Ba}$ & 378 & 27 & & 22 & 7 & & & 434 \\
\hline $\mathrm{Be}$ & & & & 71 & & & & 71 \\
\hline $\mathrm{Br}$ & 242 & 41 & & & & & & 283 \\
\hline $\mathrm{Ca}$ & 192 & 9 & 164 & 23 & 7 & & & 395 \\
\hline Cd & & & 23 & 425 & 8 & & & 456 \\
\hline $\mathrm{Ce}$ & 252 & 9 & & & & & & 261 \\
\hline $\mathrm{Cl}$ & 341 & & & & & 442 & & 783 \\
\hline Co & 385 & 9 & & & & & & 394 \\
\hline $\mathrm{Cr}$ & 404 & & & 180 & 7 & & & 591 \\
\hline Cs & 239 & 9 & 4 & & & & & 252 \\
\hline & 69 & & 26 & 47 & 7 & & & 149 \\
\hline$\ldots y$ & 116 & & & & & & & 116 \\
\hline $\mathrm{Eu}$ & 273 & & & & & & & 273 \\
\hline $\mathbf{F}$ & & & & & & 312 & 182 & 494 \\
\hline $\mathrm{Fe}$ & 521 & 9 & 4 & & & & & 534 \\
\hline $\mathrm{Ga}$ & & 97 & & & & & & 97 \\
\hline Hf & 234 & 9 & & & & & & 243 \\
\hline $\mathbf{H g}$ & & & & & & & 163 & 163 \\
\hline$K$ & 260 & 9 & 187 & & 7 & & & 463 \\
\hline $\mathbf{L a}$ & 253 & & & & & & & 253 \\
\hline $\mathbf{L i}$ & & & 112 & 337 & & & & 449 \\
\hline Lu & 65 & & & & & & & 65 \\
\hline $\mathbf{M g}$ & 95 & & 127 & 23 & 7 & & & 252 \\
\hline Mn & 555 & 9 & & 5 & & & & 569 \\
\hline Mo & & 45 & 34 & & & & & 79 \\
\hline $\mathrm{Na}$ & 457 & & 121 & 23 & 7 & & & 608 \\
\hline Nd & & 9 & & & & & & 9 \\
\hline $\mathrm{Ni}$ & 3 & 9 & & 42 & & & & 54 \\
\hline pb & & & 4 & 50 & 7 & & & 61 \\
\hline $\mathbf{P d}$ & & & & 65 & & & & 65 \\
\hline 14 & & & & 65 & & & & 65 \\
\hline Rb & 452 & 9 & & & & & & 461 \\
\hline th & & & & 65 & & & & 65 \\
\hline $\mathbf{R u}$ & & & & 65 & & & & 65 \\
\hline 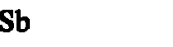 & 173 & 9 & & & & & & 182 \\
\hline $\mathbf{s c}$ & 261 & & & & & & & 261 \\
\hline
\end{tabular}


TABLE IV (cont)

\begin{tabular}{|c|c|c|c|c|c|c|c|c|}
\hline Analyais & ITNA & IENA & $\mathbf{A A}$ & FAA & ICP & IC & Other" & Total \\
\hline Se & 32 & 1 & & & 7 & & 129 & 169 \\
\hline Si & & 9 & 166 & 24 & 7 & & & 206 \\
\hline Sm & 241 & & & & & & & 241 \\
\hline Sn & & & 3 & & & & & 3 \\
\hline Sr & & 74 & & & & & & 74 \\
\hline $\mathrm{Ta}$ & 205 & 9 & & & & & & 214 \\
\hline $\mathrm{Tb}$ & 65 & 9 & & & & & & 74 \\
\hline Th & 443 & 42 & & & 35 & & & 520 \\
\hline $\mathbf{T i}$ & 177 & 65 & & & & & & 242 \\
\hline $\begin{array}{l}\mathrm{U} \\
{ }^{233} \mathrm{U}\end{array}$ & & 666 & & & 35 & & $\begin{array}{r}1182 \\
69\end{array}$ & $\begin{array}{r}1883 \\
69\end{array}$ \\
\hline${ }^{231} \mathrm{U}$ & & 69 & & & & & & 69 \\
\hline V & 429 & 9 & & & & & & 438 \\
\hline $\mathbf{w}$ & & 97 & & & & & 42 & 139 \\
\hline Yb & 87 & & & & & & & 87 \\
\hline $\mathrm{Zn}$ & 100 & 9 & 33 & 4 & 7 & & & 153 \\
\hline $\mathbf{Z r}$ & & 9 & & & & & & 9 \\
\hline pH & & & & & & & 106 & 106 \\
\hline Water Content & & & & & & & 254 & 254 \\
\hline Conductivity & & & & & & & 152 & 152 \\
\hline TDS & & & & & & & 155 & 155 \\
\hline $\mathrm{CO}_{3}$ & & & & & & & 152 & 152 \\
\hline $\mathrm{HCO}_{3}$ & & & & & & & 152 & 152 \\
\hline Hardness & & & & & & & 152 & 152 \\
\hline $\mathrm{NO}_{3}$ & & & & & & 298 & & 298 \\
\hline $\mathrm{SO}_{4}$ & & & & & & 308 & & 308 \\
\hline $\mathrm{PO}_{4}$ & & & & & & 286 & & 286 \\
\hline
\end{tabular}

"Other: As = RENA, B = TCG, F = ISE, Hg = CVAA, Se $=$ RTNA, U = DNA, ${ }^{235}$ U = DNA, W $=$ RENA, $\mathrm{pH}=$ ISE, Water = EVAP, Cond = TITR, TDS = EVAP, $\mathrm{CO}_{3}=$ TITR, $\mathrm{HCO}_{3}=$ TITR, Hard $=$ TITR (see Fig. 1 for abbreviation key).

manuscript: Wanita Eberhardt, Daryl Knab, Gloria Martinez, Diane Noveroske, James Owens, Richard Peters, and William Schweitzer. We appreciate the encouragement and support of Wayne R. Hansen and Alan K. Stoker.

\section{REFERENCES}

1. E. S. Gladney, J. W. Owens, T. C. Gunderson, and W. E. Goode, "Quality Assurance for Environmental Analytical Chemistry: 1976-1979," Los Alamos National Laboratory report, in press (1981).
2. E. S. Gladney, "Elemental Concentrations in NBS Biological and Environmental Standard Reference Materials," Anal. Chim. Acta 118, pp. 385-396 (1980).

3. E. S. Gladney, D. R. Perrin, J. W. Owens, and D. Knab, "Elemental Concentrations in the United States Geological Survey's Geochemical Exploration Reference Samples-A Review," Anal. Chem. 51, pp. 1557-1569 (1979). 
SYMBO!

\begin{tabular}{|c|c|}
\hline $\mathbf{D}$ & INSTRUMENTAL THERMAL NCUIRON ACTIVATIOH \\
\hline 0 & DELAYEO ROUTRON ANALYSHS \\
\hline$\Delta$ & ATOUIC ANSORPTIOH SPECTROSCOPY - FLAHE \\
\hline+ & AFOUIC ABSORPTHOH SPECTROSCOPY - FLANELESS \\
\hline$x$ & ION CHEOMAROGRAPHY \\
\hline 0 & tow SELCCTIVE ELCCTROOT \\
\hline$\nabla$ & IMSIMUNENTAL CPITHERMAL HEUTRON ACTIVATION \\
\hline $\mathbf{B}$ & TAOIOCHEMICAL \\
\hline$M$ & GAvenA SPECIROSCOPY \\
\hline$\bullet$ & LIOUID SCINIILLATICH COUNTING \\
\hline $\boldsymbol{\Phi}$ & PROPOATIOHAL COWNING \\
\hline $\mathbf{a}$ & THERHAL MEUTRON CAPTURE GAMUA \\
\hline 田 & EYAPORATION \\
\hline 口 & CALCUXATEO \\
\hline * & TITRINETRIC \\
\hline$\S$ & RAOIOCHEWICAL THERWAL IFUTRON ACIIYATION \\
\hline$\Psi$ & RAOIOCKEMICAL CPITHERMAL REUTRON ACTIYAIION \\
\hline 8 & COLO VAPOR ATOMIC ABSORPTION \\
\hline 5 & Wiscellanegous PROCEOURE. \\
\hline
\end{tabular}

ABBREV
ITHA
DNA
AA
FAA
IC
ISE
IENA
RC
6
LS
PC
ISC
EVAF
CALC
IITR
FTAA
PEAK
CYRA
HISC

Fig. 1. Legend of procedure symbols and abbreviations.

4. E. S. Gladney, "Compilation of Elemental Concentration Data for the United States Geological Survey's Six Geochemical Exploration Reference Materials," Los Alamos Scientific Laboratory report LA-8473-MS (1980).

5. E. S. Gladney, "Compilation of Elemental Concentration Data for Fourteen Canadian Certified Reference Materials Project Standards," Los Alamos Scientific Laboratory report LA-8382-MS (1980).

6. E. S. Gladney and W. E. Goode, "Elemental Concentrations in Eight New United States Geological Survey Rock Standards-A Review," Geostandards Newsletter 5, in press (1981).

7. F. J. Flanagan, " 1972 Values for International Geochemical Reference Samples," Geochim. Cosmochim. Acta 37, pp. 1189-1200 (1973).

8. S. Abbey, "Studies in Standard Samples for Use in the General Analysis of Silicate Rocks and Minerals, Part 6: 1979 Edition of Usable Values," Geological Survey of Canada Paper 80-14 (1980).

9. S. Abbey, A. H. Gillieson, and G. Perrault, "SY-2, SY-3, and MRG: A Report on the Collaborative
Analysis of Three Canadian Rock Samples for Use as Certified Reference Materials," Canada Centre for Mineral and Energy Teclunology, Mineral Sciences Laboratories report MRP/MSL 75-132 (TR) (1975).

10. J. C. Ingles, R. Sutarno, W. S. Bcwman, and G. H. Faye, "Radioactive Ores DH-1, DL-1, BL-1, BL-2, BL-3, and BL-4: Certified Reference Materials," CANMET report 77-64, Canada Centre for Mineral and Energy Technology (1977).

11. W. S. Bowman, G. H. Faye, R. Sutarno, J. A. McKeague, and H. Kodama," New CCRMP Reference Soils SO-1 to SO-4," Geostandards Newsletter 3, pp. 109-113 (1979).

12. S. Abbey, R. A. Meeds, and P. G. Belanger, "Reference Samples of Rocks--The Search for Best Values," Gsostandards Newsietter 3, pp. 121-133 (1979).

13. E. S. Gladney, "Compilation of Elemental Concentration Data for NBS Biological and Environmental Standard Reference Materials," Los Alamos National Laboratory report LA-8438-MS (1980).

14. S. Abbey, "Reference Materials-Rock Samples SY-2, SY-3, and MRG-1," CANMET report 79-35, Canada Centre for Mineral and Energy Technology (1979).

15. E. S. Gladney, "Comparison of Methods for Calculation of Recommended Elemental Concentrations for Canadian Certified Reference Materials Project Rock Standards SY-2, SY-3, and MRG-1," Los Alamos National Laboratory report LA-8770MS, 1981 .

16. G. A. Uriano and C. C. Gravatt, "The Role of Reference Materials and Reference Methods in Chemical Analysis," Crit. Rev. in Anal. Chem. 6, pp. 361-411 (1977).

17. E. S. Gladney, D. B. Curtis, D. R. Perrin, J. W. Owens, and W. E. Goode, "Nuciear Teclıniques for the Chemical Analysis of Environmental Materials," Los Alamos National Laboratory report LA-8192MS, pp. 36-46 (1980). 
APPENDIX A

CERTIFIED OR CONSENSUS VALUES FOR SECONDARY REFERENCE MATERIALS 


\section{REFERENCES FOR CERTIFIED OR CONSENSUS VALUES}

Reference Code

??EPA

$-A$

$-B$

$-\mathrm{C}$

??NBS-A

31 NBS-A

56 NBS

$-A$

$-B$

60 NBS-A

64 NBS

$-A$

-B

66 NBS

$-A$

$-B$

$-C$

-D

$-E$

$-F$

$-G$

$-\mathrm{H}$

$-I$

$-J$

$-\mathbf{K}$

67 IAE

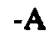

$-\mathbf{B}$

$-\mathrm{C}$

68 NBS-A
Description

Environmental Protection Agency, date and location unknown.

Arsenic Glass Fiber Filter Strips

Sulfate-Nitrate Glass Fiber Filter Strips

Lead Glass Fiber Filter Strips

National Bureau of Standards, SRM 86c, Aluminum Alloy, Analyses taken from bottle label, date unknown.

National Bureau of Standards, Certificate of Analysis, SRM 91, Opal Glass, June 15, 1931.

National Bureau of Standards, Certificates of Analysis

SRM 133A, Steel, June 8, 1956.

SRM 168, Heat Resisting Alloy, January 12, 1956.

National Bureau of Standards, Certificate of Analysis, SRMs 644-646, Spectroscopic Titanium-Base Standards, Jan. 26, 1960.

National Bureau of Standards, Certificates of Analysis.

SRM 629, Spectrographic Zinc-Base Die-Casting Alloy, April 24, 1964.

SRM 4921-C, Sodium-22, Aug. 6, 1964; dilution of stock by H-8.

National Bureau of Standards, Certificates of Analysis.

SRM U-100, Uranium Isotopic Standard, June 23, 1966.

SRM U-150, Uranium Isotopic Standard, Aug. 5, 1966.

SRM U-200, Uranium Isotopic Standard, June 1, 1966.

SRM U-350, Uranium Isotopic Standard, May 23, 1966.

SRM U-500, Uranium Isotopic Standard, May 24, 1966.

SRM U-750, Uranium Isotopic Standard, Feb. 11, 1966:

SRM U-800, Uranium Isotopic Standard, Feb. 11, 1966.

SRM U-850, Uranium Isotopic Standard, Feb. 11, 1966.

SRM U-900, Uranium Isotopic Standard, Feb. 11, 1966.

SRM U-930, Uranium Isotopic Standard, Feb. 11, 1966.

SRM 348, High Temperature Steel Alloy, Jan. 17, 1966.

International Atomic Energy Agency, Certificates.

Reference Sample S-2, Low Grade Uranium Ore, Torbenite, May 1967.

Reference Sample S-4, Low Grade Uranium Ore, Uraninite, May 1967.

Reference Sample S-3, Low Grade Uranium Ore, Carnotite, May 1967.

National Bureau of Standards, Certificate of Analysis, SRM 4958, Gamma

Ray Standard, Radium-226, March 1, 1968. 
TABLE A-I (cont)

Reference Code

69 NBS

$-\mathbf{A}$

$-B$

$-C$

$-D$

$-E$

$-F$

70 NBS

$-A$

$-B$

$-\mathrm{C}$

$-\mathrm{D}$

71 NBS-A

72 NBS

$-A$

-B

$-\mathrm{C}$

$-D$

$-E$

$-\mathrm{F}$

$-G$

73 FLA-A

74 EPA-A

74 JEN-A

74 NBS

$-\mathbf{A}$

$-B$

$-C$

$-\mathrm{D}$

$-E$

$-F$

$-G$

75 EPA-A
Description

National Bureau of Standards, Certificates of Analysis.

SRM U-010, Uranium Isotopic Standards, April 21, 1969.

SRM U-015, Uranium Isotopic Standards, April 21, 1969.

SRM U-020, Uranium Isotopic Standards, April 21, 1969.

SRM 950a, Uranium Oxide, Oct. 27, 1969.

SRM 1090-1092, Oxygen in Ferrous Materials, April 2, 1969.

SRM 1093, Oxygen in Valve Steel, March 4, 1969.

National Bureau of Standards, Certificates of Analysis.

SRM U-0002, Uranium Isotopic Standard, July 30, 1970.

SRM U-970, Uranium Isotopic Standard, July 9, 1970.

SRM 83c, Arsenic Trioxide, April 16, 1970.

SRM 53e, Lead-Base Bearing Metal, Jan. 20, 1970.

National Bureau of Standards, Certificate of Analysis, SRM 79a, Fluorspar, Dec. 6, 1971.

National Bureau of Standards, Certificates of Analysis.

SRM 610, Trace Elements in Glass, Aug. 8, 1972.

SRM 612, Trace Elements in Glass, Aug. 8, 1972.

SRM 614, Trace Elements in Glass, Aug. 8, 1972.

SRM 616, Trace Elements in Glass, Aug. 8, 1972.

SRM 1577, Bovine Liver, April 15, 1972.

SRM 120b, Phosphate Rock, July 31, 1972.

SRM 1135, High Silicon Steel, July 27, 1972.

F. J. Flanagen, "1972 Values for International Geochemical Reference Samples," Geochim. Cosmochim. Acta, 37: 1189-1200 (1973).

Environmental Protection Agency, "EPA Quality Control Samples for Mineral Analyses," Quality Assurance Branch, EMSL, 1974.

Junta de Energia Nuclear, Certificado de Analisis, Madrid, Spain, Nov. 1974.

National Bureau of Standards, Certificates of Analyses.

SRM 1632, Trace Elements in Coal, Oct. 1, 1974.

SRM 1642, Mercury in Water-Trace, Aug. 16, 1974.

SRM 193, Potassium Nitrate, Nov. 14, 1974.

SRM 194, Ammonium Dihydrogen Phosphate, Jan. 8. 1974.

SRM 113a and 329, Zinc Concentrates, May 6, 1974.

SRM 1197-1201, High Temperature Alloys, Aug. 17, 1974.

SRM 1631, Sulfur in Coal, Dec. 6, 1974.

Environmental Protection Agency, "EPA Quality Control Samples for Trace Metals Analyses," Quality Assurance Branch, EMSL, 1975. 
Reference Code

75 NBS

$-A$

$-B$

$-\mathrm{C}$

$-\mathrm{D}$

$-\mathrm{E}$

76 AND-A

76 EPA-A

76 MIL-A

76 NBS

$-A$

-B

$-C$

$-\mathrm{D}$

77 ING-A

77 EPA-A

77 NBS

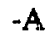

-B

78 EPA

$-\mathbf{A}$

$-\mathbf{B}$

$-\mathrm{C}$
Description

National Bureau of Standards, Certificates of Analysis.

SRM 1633, Trace Elements in Coal Fly Ash, March 7, 1975.

SRM 1641, Mercury in Water-Concentrate, March S, 1975.

SRM 2671, Freeze Dried Urine Certified for Fluoride, Oct. 1, 1975.

SRM 633-639, Portland Cements, Feb. 1975.

SRM 1261-1265, Steel, Feb. 24, 1975.

D. H. Anderson, J. J. Murphey, W. W. White, "Gelatin Multicomponent Trace Element Reforence Material," Anal. Chem. 48, 116-117 (1976).

Environmental Protection Agency, Quality Control Check Samples for Trace Metals, Quality Assurance Branch, EMSL, April 1976.

H. T. Millard, "Determination of Uranium and Thorium in USGS Standard Rocks by the Delayed Neutron Technique: USGS Professional Paper 840. pp. 61-65, 1976.

National Bureau of Standards, Certificates of Analysis.

SRM 1570, Trace Elements in Spinach, Sept. 1, 1976.

SRM 1573, Tomato Leaves, Oct. 18, 1976.

SRM 1575, Pine Needles, Oct. 18, 1976.

SRM 1569, Brewer's Yeast, Sept. 7, 1976.

J. C. Ingles, R. Sutarno, W. S. Bowman, and G. H. Faye, "Radioactive Ores DH-1, DL-1, BL-1, BL-2, BL-3, and BL-4-Certified Reference Materials," CANMET report 77-64, 1977.

Environmental Protection Agency, Water Supply Quality Control Check Samples for Nitrate/Fluoride, Quality Assurance Branch, EMSL, June 1977.

National Bureau of Standards, Certificates of Analysis.

SRM 1571, Orchard Leaves, Aug. 31, 1977.

SRM 1642A, Mercury in Water-ng/ml, Aug. 24, 1977.

Environmental Protection Agency, Quality Assurance Branch, EMSL. Quality Control Samples for Nutrients, May 1978.

Quality Control Samples for Mineral Analyses, April 1978.

Water Supply Quality Control Check Samples for Trace Metals, March 1978. 
Reference Code

78 NBS

$-A$

$-B$

$-C$

$-D$

$-E$

$-F$

78 BOW-A

79 EPA-A

79 FAY-A

79 GLA-A

79 H-8

79 NBS

$-A$

-B

$-\mathrm{C}$

79 SPE-A

80 EML-A

80 EPA-A

80 GLA-A
Description

National Bureau of Standards, Certificates of Analysis.

SRM 1632A, Trace Elements in Coal (Bituminous), Jan. 23, 1978.

SRM 1635, Trace Elements in Coal (Subbituminous), Jan. 23, 1978.

SRM 1648, Urban Particulate Matter, Nov. 15, 1978.

SRM 1645, River Sediment, Nov. 16, 1978.

SRM 1567, Wheat Flour, Jan. 3, 1978.

SRM 1568, Rice Flour, Jan. 3, 1978.

W. S. Bowman, G. H. Faye, R. Sutarno, J. A. McKeague, and H. Kodama, “New CCRMP Reference Soils SO-1 to SO-4," Geostandards Newsletter 3: 109-113 (1979).

Environmental Protection Agency, Quality Control Check Samples for Trace Metals, Quality Assurance Branch, EMSL, Mar. 1979.

G. H. Faye, W. S. Bowman, and R. Sutarno, "Uranium Ore BL-5-A Certified Reference Material," CANMET report 79-4, Feb. 1979.

E. S. Gladney, D. R. Perrin, J. W. Owens, and D. Knab, "Elemental Concentrations in the United States Geological Survey's Geochemical Exploration Reference Samples-A Review," Anal. Chem. 51: 1557-1569 (1979).

In House Quality Control, Environmental Surveillance Group, 1979.

National Bureau of Standards, Certificates of Analysis.

SRM 4926-C, Hydrogen-3, Jan. 1979; Dilution of Master Solution by H-8. SRM 1633a, Trace Elements in Coal Fly Ash, April 18, 1979.

SRM 1566, Oyster Tissue, Dec. 12, 1979.

R. R. Spencer and D. E. Erdmann, "Azomethine H Colorimetric Method for Determining Dissolved Boron in Water," Environ. Sci. Technol. 13: 954-956 (1979).

Environmental Measurements Laboratory, 1980.

Environmental Protection Agency, Quality Assurance Division, EMSL-LV, "Calibration Certificate, Beryllium-8," May 28, 1980.

E. S. Gladney, "Elemental Concentrations in NBS Biological and Environmental Standard Reference Materials," Anal. Chim. Acta 118: 385-396 (1980), "Compilation of Elemental Concentration Data for NBS Biological and Environmental Standard Reference Materials," Los Alamos Scientific Laboratory report LA-8438-MS, July 1980. 
TABLE A-I (cont)

Reference Code

-B

$-C$

-D

$80 \mathrm{H}-8$

80 NBS-A

80 STE-A

81 GLA-A

- B

\section{Description}

E. S. Gladney, "Compilation of Elemental Concentration Data for the United States Geological Survey's Six Geochemical Exploration Reference Materials," Los Alamos Scientific Laboratory report LA-8473-MS, Aug. 1980.

E. S. Gladney, D. R. Perrin, W. K. Hensley, "Determination of Uranium in NBS Biological Standard Reference Materials by Delayed Neutron Assay," J. Radioanal. Chem. 59: 249-251 (1980).

E. S. Gladney, D. R. Perrin, J. P. Bologna, and C. L. Warner, "Evaluation of a Boron-Filtered Epithermal Neutron Irradiation Facility," Anal. Chem. 52: $2128-2132$ (1980).

In House Quality Control, Environmental Surveillance Group, 1980.

National Bureau of Standards, Certificate of Analysis, SRM 1643a, Trace Elements in Water, March 3, 1980.

H. F. Steger and W. S. Bowman, "DL-1A: A Certified Uranium-Thorium Reference Ore," CANMET report 80-10E, May 1980.

E. S. Gladney and W. E. Goode, "Elemental Concentrations in Eight New United States Geological Survey Rock Standards-A Review," Geostandards Newsletter 5: 31-64 (1981).

E. S. Gladney, "Comparison of Methods for Calculation of Recommended Elemental Concentration Values for Canadian Certified Reference Materials Project Rock Standards SY-2, SY-3, and MRG-1," Los Alamos National Laboratory report LA-8770-MS, 1981. 
TABLE A-II

\section{CERTIFIED OR CONSENSUS VALUES FOR SECONDARY REFERENCE MATERIALS}

\begin{tabular}{|c|c|c|c|c|c|}
\hline $\begin{array}{l}\text { SANPLE } \\
\text { NUMBER }\end{array}$ & $\begin{array}{l}\text { CERTIFIED } \\
\text { VALUE } \\
\text { NAME }\end{array}$ & $\begin{array}{l}\text { CERT IFIED } \\
\text { VALUE }\end{array}$ & $\begin{array}{l}\text { CERTIFIED } \\
\text { VALUE } \\
\text { UNCERTAINTY }\end{array}$ & $\begin{array}{l}\text { CERTIF IED } \\
\text { VALUE } \\
\text { UNITS }\end{array}$ & REFERENCE \\
\hline \multirow{2}{*}{80.00500} & $-\infty$ & $--m-n$ & - - n-m-n & 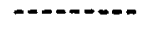 & $-\infty+\infty-\infty$ \\
\hline & TDS & 500. & 20. & PPM & $80 \mathrm{H}-8$ \\
\hline \multicolumn{6}{|l|}{80.00501} \\
\hline & COND & 1000 & 100 . & UMHO" & $80 \mathrm{H}-8$ \\
\hline \multicolumn{6}{|l|}{80.00503} \\
\hline & $\begin{array}{l}\mathrm{F} \\
\mathrm{NO3}\end{array}$ & $\begin{array}{l}.36 \\
.97\end{array}$ & & $\begin{array}{l}P P M \\
P P M\end{array}$ & $\begin{array}{l}\text { 77EPA-A } \\
\text { 77EPA-A }\end{array}$ \\
\hline \multicolumn{6}{|l|}{80.00504} \\
\hline & $\begin{array}{l}\text { F } \\
\text { NO3 }\end{array}$ & $\begin{array}{r}2.3 \\
44.7\end{array}$ & & $\begin{array}{l}\text { PPM } \\
\text { PPM }\end{array}$ & $\begin{array}{l}\text { 77ЕРА-А } \\
\text { 77ЕРА-А }\end{array}$ \\
\hline \multicolumn{6}{|c|}{80.00505} \\
\hline & $\begin{array}{l}A G \\
A S \\
B A \\
B E \\
C D \\
C 0 \\
C R \\
C U \\
F E \\
M N \\
M O \\
N I \\
P B \\
S E \\
S R \\
Y \\
Z N\end{array}$ & $\begin{array}{c}2.8 \\
76 . \\
46 . \\
19 . \\
10 . \\
19 . \\
17 . \\
18 . \\
88 . \\
31 . \\
95 . \\
55 . \\
27 . \\
11 . \\
239 . \\
53 . \\
72 .\end{array}$ & $\begin{array}{l}73 \\
7 . \\
2 . \\
2 . \\
1 . \\
2 . \\
2 . \\
2 . \\
4 . \\
2 . \\
6 . \\
3 . \\
1 . \\
1 . \\
5 . \\
3 . \\
4 .\end{array}$ & $\begin{array}{l}\text { PPB } \\
P P B \\
P P B \\
P P B \\
P P B \\
P P B \\
P P B \\
\text { PPB } \\
P P B \\
\text { PPB } \\
\text { PPB } \\
\text { PPB } \\
\text { PPB } \\
\text { PPB } \\
\text { PPB } \\
\text { PPB } \\
\text { PPB }\end{array}$ & $\begin{array}{l}80 N B S-A \\
80 N B S-A \\
80 N B S-A \\
80 N B S-A \\
80 N B S-A \\
80 N B S-A \\
80 N B S-A \\
80 N B S-A \\
80 N B S-A \\
80 N B S-A \\
\text { 80NBS-A } \\
80 N B S-A \\
\text { 80NBS-A } \\
\text { 80NSS-A } \\
\text { 80NBS-A } \\
\text { 80NBS-A } \\
\text { 80NBS-A }\end{array}$ \\
\hline
\end{tabular}

80.00509

\begin{tabular}{|c|c|c|c|}
\hline $\begin{array}{l}A G \\
A S \\
B R \\
C A \\
C D \\
C L \\
C D \\
C R \\
C U \\
F \\
F E \\
H G \\
I \\
K \\
M G \\
M N \\
N A \\
\text { II }\end{array}$ & $\begin{array}{c}.89 \\
13.4 \\
55 . \\
1500 . \\
3.5 \\
1 . \\
.4 \\
.69 \\
63 . \\
5.2 \\
195 . \\
.057 \\
2.8 \\
9690 . \\
1280 . \\
17.5 \\
5100 . \\
1.03 \\
8100 .\end{array}$ & $\begin{array}{c}. .09 \\
1.9 \\
200 . \\
.4 \\
.27 \\
3.5 \\
34 . \\
.015 \\
50 . \\
90 . \\
1.2 \\
300 . \\
.19\end{array}$ & $\begin{array}{l}\text { PPM } \\
\text { PPM } \\
\text { PPM } \\
\text { PPM } \\
\text { PPM } \\
\% \\
\text { PPM } \\
\text { PPM } \\
\text { PPM } \\
\text { PPM } \\
\text { PPM } \\
\text { PPM } \\
\text { PPM } \\
\text { PPM } \\
\text { PPM } \\
\text { PPM } \\
\text { PPM } \\
\text { PPM } \\
\text { PPM }\end{array}$ \\
\hline
\end{tabular}


TABLE A-II (cont)

\begin{tabular}{|c|c|c|c|c|c|}
\hline $\begin{array}{l}\text { SAMPLE } \\
\text { NUMBER }\end{array}$ & $\begin{array}{l}\text { CERTIF IED } \\
\text { VALUE } \\
\text { NAME }\end{array}$ & $\begin{array}{l}\text { CERTIFIED } \\
\text { VALUE }\end{array}$ & $\begin{array}{l}\text { CERTIFIED } \\
\text { VALUE } \\
\text { UNCERTAINTY }\end{array}$ & $\begin{array}{l}\text { CERTIFIED } \\
\text { VALUE } \\
\text { UNITS }\end{array}$ & REFERENCE \\
\hline .06 & $\begin{array}{l}P B \\
R B \\
S \\
S E \\
\text { SR } \\
T H \\
U \\
V \\
\text { ZN }\end{array}$ & $\begin{array}{c}.48 \\
4.45 \\
7600 . \\
2.1 \\
10.36 \\
.1 \\
.116 \\
2.8 \\
852 .\end{array}$ & $\begin{array}{c}.04 \\
.09 \\
.5 \\
.56 \\
.006 \\
14 .\end{array}$ & $\begin{array}{l}P P M \\
P P M \\
P P M \\
P P M \\
P P M \\
P P M \\
P P M \\
P P M \\
P P M\end{array}$ & $\begin{array}{l}\text { 79NBS-C } \\
\text { 79NES-C } \\
\text { 79NBS-C } \\
\text { 79NBS-C } \\
\text { 79NBS-C } \\
\text { 79NBS-C } \\
\text { 79NBS-C } \\
\text { 79NBS-C } \\
\text { 79NBS-C }\end{array}$ \\
\hline 80.00510 & $\mathrm{H}-3$ & 800. & & $\mathrm{PCI} / \mathrm{L}$ & 79NBS-A \\
\hline 80.00511 & $\mathrm{H}-3$ & 1600. & & PCI/L. & 79NBS-A \\
\hline 80.00512 & $\mathrm{H}-3$ & 3200 & & PCI/L & 79NBS-A \\
\hline 80.00513 & $H-3$ & 8000 . & & PCI/L & 79NBS-A \\
\hline 80.00514 & $\mathrm{H}-3$ & 16000. & & $\mathrm{PCI} / \mathrm{L}$ & 79NBS-A \\
\hline 80.00515 & $\begin{array}{l}A L \\
A S \\
B \\
B A \\
B E \\
C \\
C A \\
C E \\
C L \\
C O \\
C R \\
C S \\
C U \\
D Y \\
E R \\
E U \\
F \\
F E \\
G A \\
G D \\
H F \\
H O \\
K \\
L A \\
L I \\
L U \\
M G\end{array}$ & $\begin{array}{c}6.36 \\
18 . \\
87 . \\
470 . \\
20 . \\
1600 . \\
5.71 \\
210 . \\
140 . \\
11 . \\
10 . \\
2.4 \\
6 . \\
20 . \\
12 . \\
2.4 \\
4800 . \\
4.42 \\
29 . \\
11 . \\
8 . \\
5 . \\
3.71 \\
79 . \\
95 . \\
2.8 \\
1.63\end{array}$ & $\begin{array}{c}.11 \\
2 . \\
15 . \\
40 . \\
6 . \\
100 . \\
.11 \\
60 . \\
40 . \\
4 . \\
3 . \\
.4 \\
3 . \\
1 . \\
.4 \\
500 . \\
.12 \\
4 . \\
1 . \\
1 . \\
2 . \\
.12 \\
18 . \\
8 . \\
.5 \\
.06\end{array}$ & 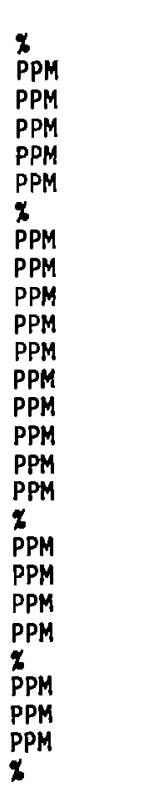 & $\begin{array}{l}\text { 81GLA-B } \\
\text { 81GLA-B } \\
\text { 81GLA-B } \\
\text { 81GLA-B } \\
\text { 81GLA-B } \\
\text { B1GLA-B } \\
\text { 81GLA-B } \\
\text { 81GLA-B } \\
\text { 81GLA-B } \\
\text { 81GLA-B } \\
\text { 81GLA-B } \\
\text { 81GLA-B } \\
\text { 81GLA-B } \\
\text { 81GLA-B } \\
\text { 81GLA-B } \\
\text { 81GLA-B } \\
\text { 81GLA-B } \\
\text { 81GLA-B } \\
\text { 81GLA-B } \\
\text { 81GLA-B } \\
\text { 81GLA-B } \\
\text { 81GLA-B } \\
\text { 81GLA-B } \\
\text { 81GLA-B } \\
\text { 81GLA-B } \\
\text { 81GLA-B } \\
\text { 81GLA-B }\end{array}$ \\
\hline
\end{tabular}


TABLE A-II (cont)

\begin{tabular}{|c|c|c|c|c|c|}
\hline \multirow{3}{*}{$\begin{array}{c}\text { SANPLE } \\
\text { NUMBER } \\
80.00515\end{array}$} & $\begin{array}{l}\text { CERTIFIED } \\
\text { VALUE } \\
\text { NAME }\end{array}$ & $\begin{array}{l}\text { CERTIF IED } \\
\text { VALUE }\end{array}$ & $\begin{array}{l}\text { CERTIFIED } \\
\text { VALUE } \\
\text { UNCERTAINTY }\end{array}$ & $\begin{array}{l}\text { CERTIFIED } \\
\text { VALUE } \\
\text { UNITS }\end{array}$ & REFERENCE \\
\hline & $\ldots$ & $--\infty+\infty-0$ &...-- & - & - \\
\hline & $\begin{array}{l}M N \\
N A \\
N B \\
N D \\
N I \\
0 \\
P \\
P B \\
P R \\
R B \\
S \\
S B \\
S C \\
S I \\
S M \\
S N \\
S R \\
T A \\
T B \\
T H \\
T I \\
T L \\
T M \\
U \\
V \\
Y \\
Y B \\
Z N \\
Z R\end{array}$ & $\begin{array}{c}2500 . \\
3.21 \\
25 . \\
76 . \\
10 . \\
45 . \\
1900 . \\
83 . \\
14 . \\
217 . \\
190 . \\
250 . \\
7.3 \\
28.06 \\
15 . \\
3.3 \\
271 . \\
1.9 \\
2.5 \\
360 . \\
900 . \\
1.5 \\
2.1 \\
280 . \\
50 . \\
130 . \\
17 . \\
250 . \\
280 .\end{array}$ & $\begin{array}{c}100 . \\
.1 \\
7 . \\
20 . \\
4 . \\
1.2 \\
100 . \\
12 . \\
4 . \\
14 . \\
90 . \\
80 . \\
1.1 \\
.19 \\
2 . \\
.9 \\
17 . \\
.2 \\
.8 \\
70 . \\
120 . \\
.4 \\
.4 \\
25 . \\
8 . \\
20 . \\
3 . \\
20 . \\
30 .\end{array}$ & $\begin{array}{l}P P M \\
x \\
P P M \\
P P M \\
P P M \\
x \\
P P M \\
P P M \\
P P M \\
P P M \\
P P M \\
P P B \\
P P M \\
\% \\
P P M \\
P P M \\
P P M \\
P P M \\
P P M \\
P P M \\
P P M \\
P P M \\
P P M \\
P P M \\
P P M \\
P P M \\
P P M \\
P P M \\
P P M\end{array}$ & $\begin{array}{l}\text { 81GLA-B } \\
81 G L A-B \\
81 G L A-B \\
81 G L A-B \\
81 G L A-B \\
81 G L A-B \\
81 G L A-B \\
81 G L A-B \\
81 G L A-B \\
\text { 81GLA-B } \\
\text { 81GLA-B } \\
\text { 81GLA-B } \\
81 G L A-B \\
\text { 81GLA-B } \\
\text { 81GLA-B } \\
\text { 81GLA-B } \\
\text { 81GLA-B } \\
\text { 81GLA-B } \\
\text { 81GLA-B } \\
\text { 81GLA-B } \\
\text { 81GLA-B } \\
\text { 81GLA-B } \\
\text { 81GLA-B } \\
\text { 81GLA-B } \\
\text { 81GLA-B } \\
\text { 81GLA-B } \\
\text { 81GLA-B } \\
\text { 81GLA-B } \\
\text { 81GLA-B }\end{array}$ \\
\hline
\end{tabular}

80.00516

$\begin{array}{lc}\text { AL } & 6.22 \\ \text { AS } & 19 . \\ \text { B } & 102 . \\ \text { BA } & 460 . \\ \text { BE } & 20 . \\ \text { C } & 1300 . \\ \text { CA } & 5.9 \\ \text { CE } & 2110 . \\ \text { CL } & 140 . \\ \text { CO } & 11 . \\ \text { CS } & 2.4 \\ \text { CU } & 17 . \\ \text { DY } & 89 . \\ \text { ER } & 43 . \\ \text { EU } & 14 . \\ \text { F } & 7100 . \\ \text { FE } & 4.53 \\ \text { GA } & 28 . \\ \text { GD } & 54 . \\ \text { GE } & 1.3 \\ \text { HF } & 10 . \\ \text { HO } & 19 .\end{array}$

\begin{tabular}{|c|c|c|}
\hline $\begin{array}{c}.15 \\
3 . \\
13 . \\
50 . \\
6 . \\
200 . \\
.12 \\
710 . \\
30 . \\
4 . \\
.5 \\
4 . \\
26 . \\
6 . \\
3 . \\
600 . \\
.11 \\
4 . \\
2 . \\
.2 \\
1 . \\
8 .\end{array}$ & $\begin{array}{l}\% \\
\text { PPM } \\
\text { PPM } \\
\text { PPM } \\
\text { PPM } \\
\text { PPM } \\
\% \\
\text { PPM } \\
\text { PPM } \\
\text { PPM } \\
\text { PPM } \\
\text { PPM } \\
\text { PPM } \\
\text { PPM } \\
\text { PPM } \\
\text { PPM } \\
\% \\
\text { PPM } \\
\text { PPM } \\
\text { PPM } \\
\text { PPM } \\
\text { PPM }\end{array}$ & $\begin{array}{l}\text { 81GLA-B } \\
\text { 81GLA-B } \\
\text { 81GLA-B } \\
\text { 81GLA-B } \\
\text { 81GLA-B } \\
\text { 81GLA-B } \\
\text { 81GLA-B } \\
\text { 81GLA-B } \\
\text { 81GLA-B } \\
\text { 81GLA-B } \\
\text { 81GLA-B } \\
\text { 81GLA-B } \\
\text { 81GLA-B } \\
\text { 81GLA-B } \\
\text { 81GLA-B } \\
\text { 81GLA-B } \\
\text { 81GLA-B } \\
\text { 81GLA-B } \\
\text { 81GLA-B } \\
\text { 81GLA-B } \\
\text { 81GLA-B } \\
\text { 81GLA-B }\end{array}$ \\
\hline
\end{tabular}


TABLE A-II (cont)

\begin{tabular}{|c|c|c|c|c|c|}
\hline $\begin{array}{l}\text { SAMPLE } \\
\text { NUMBER }\end{array}$ & $\begin{array}{l}\text { CERTIFIED } \\
\text { VALUE } \\
\text { NAME }\end{array}$ & $\begin{array}{l}\text { CERTIF IED } \\
\text { VALUE }\end{array}$ & $\begin{array}{l}\text { CERTIFIED } \\
\text { VALUE } \\
\text { UNCERTAINTY }\end{array}$ & $\begin{array}{l}\text { CERTIFIED } \\
\text { VALUE } \\
\text { UNITS }\end{array}$ & REFERENCE \\
\hline \multirow{29}{*}{80.00516} & & -1 & & & \\
\hline & $k$ & 3.5 & .1 & $\%$ & 81GLA-B \\
\hline & LA & 1220. & 360 . & PPM & 81GLA-B \\
\hline & LI & 92. & 7. & PPM & 81GLA-B \\
\hline & $L_{M G}^{L U}$ & $\begin{array}{l}6 . \\
1.62\end{array}$ & ${ }^{3 .} .08$ & PPM & $\begin{array}{l}\text { 81GLA-B } \\
\text { 81GLA-B }\end{array}$ \\
\hline & MN & 2600 & 100. & PPM & 81GLA-B \\
\hline & NA & 3.06 & .1 & $\%$ & 81GLA-B \\
\hline & NB & 150. & 40. & PPM & 81GLA-B \\
\hline & ND & 610. & 220. & PPM & 81GLA-B \\
\hline & $\begin{array}{l}N I \\
0\end{array}$ & $\begin{array}{l}11 . \\
45 .\end{array}$ & ${ }^{4.4}$ & PPM & $\begin{array}{l}\text { 81GLA-B } \\
\text { 81GLA-B }\end{array}$ \\
\hline & p & 2400 . & 100. & PPM & 81GLA-B \\
\hline & PB & 130. & 20. & PPM & 81GLA-B \\
\hline & $\begin{array}{l}P R \\
P R\end{array}$ & 122. & 4. & $\begin{array}{l}\text { PPM } \\
\text { PPM }\end{array}$ & $\begin{array}{l}\text { 81GLA-B } \\
\text { 81GLA-B }\end{array}$ \\
\hline & $\begin{array}{l}\mathrm{RB} \\
\mathrm{S}\end{array}$ & $\begin{array}{l}200 . \\
500 .\end{array}$ & 160. & PPM & 81GLA-B \\
\hline & SB & 300. & 80. & PPB & 31GLA-B \\
\hline & $\mathrm{SC}$ & 8. & 3.17 & PPM & 81GLA-B \\
\hline & $\begin{array}{l}\text { SI } \\
\text { SM }\end{array}$ & & 30.17 & PPM & $\begin{array}{l}\text { 81GLA-B } \\
\text { 81GLA-B }\end{array}$ \\
\hline & $S N$ & 5. & 2. & PPM & 81GLA-B \\
\hline & $S R$ & 300. & 22. & PPM & 81GLA-B \\
\hline & TB & 13. & $\begin{array}{r}4 . \\
80\end{array}$ & $\begin{array}{l}\text { PPM } \\
\text { PPM }\end{array}$ & $\begin{array}{l}\text { 81GLA-B } \\
\text { 81GLA-B }\end{array}$ \\
\hline & . TI & 900. & 120. & PPM & 81GLA-B \\
\hline & $\mathrm{TL}$ & 1.6 & .6 & PPM & 81GLA-B \\
\hline & $T M$ & 8. & 3. & PPM & 81GLA-B \\
\hline & $u$ & 630. & 60 . & PPM & 81GLA-B \\
\hline & $v$ & 50. & 9. & PPM & $81 G L A-B$ \\
\hline & $Y$ & 730 . & 80. & PPM & 81GLA-B \\
\hline & YB & 60. & 19. & PPM & 81GLA-B \\
\hline & $\mathrm{ZN}$ & 245 & 17. & PPM & 81GLA-B \\
\hline & $\mathrm{ZR}$ & 320 & 40. & PPM & 81GLA-B \\
\hline
\end{tabular}

80.00517

\begin{tabular}{|c|c|c|c|c|}
\hline $\begin{array}{l}A L \\
B A \\
C \\
C A \\
C E \\
C L \\
C D \\
C R \\
C S \\
C U \\
D Y \\
E R \\
E U \\
F \\
F E \\
G A \\
G D \\
H F\end{array}$ & $\begin{array}{c}4.5 \\
68 . \\
3100 . \\
10.48 \\
28 . \\
150 . \\
86 . \\
430 . \\
600 . \\
133 . \\
3.4 \\
1 . \\
1.4 \\
240 . \\
12.55 \\
18 . \\
4 . \\
3.5\end{array}$ & $\begin{array}{c}29.14 \\
100 . \\
.22 \\
6 . \\
40 . \\
12 . \\
80 . \\
300 . \\
12 . \\
.9 \\
.1 \\
.1 \\
50 . \\
.27 \\
3 . \\
1 . \\
.5\end{array}$ & $\begin{array}{l}\% \\
\text { PPM } \\
\text { PPM } \\
\% \\
\text { PPM } \\
\text { PPM } \\
\text { PPM } \\
\text { PPM } \\
\text { PPB } \\
\text { PPM } \\
\text { PPM } \\
\text { PPM } \\
\text { PPM } \\
\text { PPM } \\
\% \\
\text { PPM } \\
\text { PPM } \\
\text { PPM }\end{array}$ & $\begin{array}{l}\text { 81GLA-B } \\
\text { 81GLA-B } \\
\text { 81GLA-B } \\
\text { 81GLA-B } \\
\text { 81GLA-B } \\
\text { 81GLA-B } \\
\text { 81GLA-B } \\
\text { 81GLA-B } \\
\text { 81GLA-B } \\
\text { 81GLA-B } \\
\text { 81GLA-B } \\
\text { 81GLA-B } \\
\text { 81GLA-B } \\
\text { 81GLA-B } \\
\text { 81GLA-B } \\
\text { 81GLA-B } \\
\text { 81GLA-B } \\
\text { 81GLA-B }\end{array}$ \\
\hline
\end{tabular}


TABLE A-II (cont)

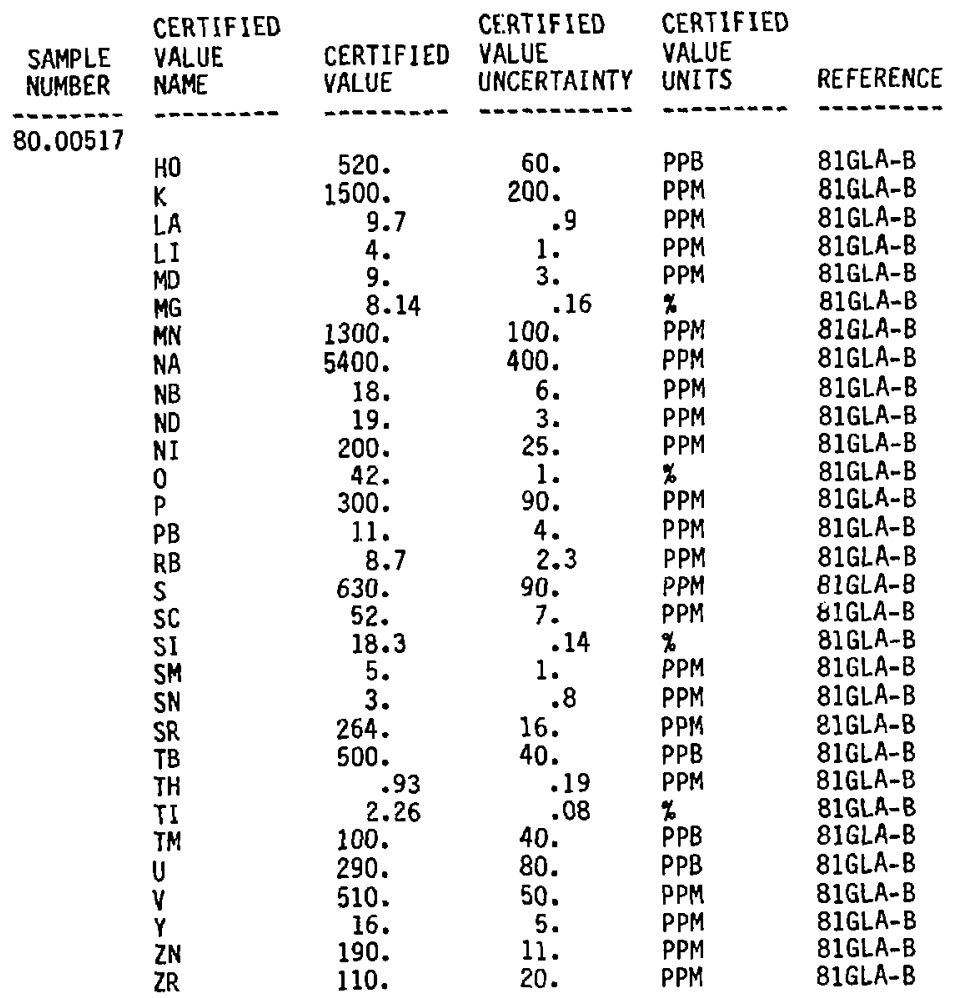

80.00518

$\begin{array}{lc}\text { AL } & 7.4 \\ \text { BA } & 420 . \\ \text { BI } & 20 . \\ \text { CA } & 1.9 \\ \text { CD } & 360 . \\ \text { CO } & 26 . \\ \text { CU } & 63 . \\ \text { FE } & 4.8 \\ \text { K } & 1 . \\ \text { MG } & 1.2 \\ \text { MD } & 34 . \\ \text { NA } & 3.9 \\ \text { PB } & 71 . \\ \text { RA-226 } & 75 . \\ \text { S } & 2700 . \\ \text { SI } & 30.4 \\ \text { SR } & 50 . \\ \text { TH } & 15 . \\ \text { U } & 220 . \\ V & 210 .\end{array}$

$\begin{array}{ll}\% & \text { 77ING-A } \\ \text { PPM } & \text { 77ING-A } \\ \text { PPM } & \text { 77ING-A } \\ \% & \text { 77ING-A } \\ \text { PPB } & \text { 77INGA } \\ \text { PPM } & \text { 77ING-A } \\ \text { PPM } & \text { 77ING-A } \\ \% & \text { 77ING-A } \\ \% & \text { 77ING-A } \\ \% & \text { 77ING-A } \\ \text { PPM } & \text { 771NG-A } \\ \% & \text { 77ING-A } \\ \text { PPM } & \text { 771NG-A } \\ \text { PCI/G } & \text { 77ING-A } \\ \text { PPM } & \text { 77NG-A } \\ \% & \text { 77ING-A } \\ \text { PPM } & \text { 77ING-A } \\ \text { PPM } & \text { 77ING-A } \\ \text { PPM } & \text { 77ING-A } \\ \text { PPM } & \text { 77ING-A }\end{array}$


TABLE A-II (cont)

\begin{tabular}{|c|c|c|c|c|c|}
\hline \multirow{3}{*}{$\begin{array}{c}\text { SAMPLE } \\
\text { NUMBER } \\
80.00518 \\
80.00519\end{array}$} & $\begin{array}{l}\text { CERTIFIED } \\
\text { VALUE } \\
\text { NAME }\end{array}$ & $\begin{array}{l}\text { CERTIFIED } \\
\text { VALUE }\end{array}$ & $\begin{array}{l}\text { CERTIFIED } \\
\text { VALUE } \\
\text { UNCERTAINTY }\end{array}$ & $\begin{array}{l}\text { CERTIFIED } \\
\text { VALUE } \\
\text { UNITS } \\
-\end{array}$ & REFERENCE \\
\hline & & & & & \\
\hline & $\begin{array}{l}A L \\
B A \\
B I \\
C A \\
C D \\
C D \\
C U \\
F E \\
K \\
M G \\
N A \\
P B \\
R A-226 \\
S \\
S I \\
S R \\
U \\
V\end{array}$ & $\begin{array}{c}7.3 \\
350 . \\
20 . \\
2.8 \\
250 . \\
25 . \\
78 . \\
5.4 \\
6600 . \\
1.4 \\
4.2 \\
922 . \\
1490 . \\
3300 . \\
28.1 \\
70 . \\
4530.005 \\
841 .\end{array}$ & 30. & $\begin{array}{l}\% \\
P P M \\
P P M \\
\% \\
P P B \\
P P M \\
P P M \\
\% \\
P P M \\
x \\
\% \\
P P M \\
P C I / G \\
P P M \\
x \\
P P M \\
P P M \\
P P M\end{array}$ & $\begin{array}{l}77 \text { ING-A } \\
771 \mathrm{NG}-A \\
77 \mathrm{NG}-A \\
77 \mathrm{ING}-A \\
77 \mathrm{ING}-A \\
77 \mathrm{NG}-A \\
77 \mathrm{NG}-A \\
771 \mathrm{NG}-A \\
77 \mathrm{NG}-A \\
77 \mathrm{NG}-A \\
77 \mathrm{NG}-A \\
77 \mathrm{NG}-A \\
77 \mathrm{NG}-A \\
771 \mathrm{NG}-A \\
77 \mathrm{NG}-A \\
77 \mathrm{ING}-A \\
77 \mathrm{NG}-A \\
77 \mathrm{ING}-A\end{array}$ \\
\hline \multicolumn{6}{|c|}{80.00520} \\
\hline & $\begin{array}{l}A L \\
B A \\
B I \\
C A \\
C D \\
C D \\
C U \\
F E \\
K \\
M G \\
M 0 \\
N A \\
P B \\
R A-226 \\
S \\
S I \\
S R \\
U \\
V\end{array}$ & $\begin{array}{c}7.3 \\
370 . \\
20 . \\
2.9 \\
310 . \\
23 . \\
91 . \\
5.3 \\
6600 . \\
1.4 \\
41 . \\
4.2 \\
1710 . \\
3330 . \\
3600 . \\
27.8 \\
70 . \\
1.02 \\
834 .\end{array}$ & .01 & $\begin{array}{l}\% \\
\text { PPM } \\
P P M \\
x \\
\text { PPB } \\
P P M \\
P P M \\
x \\
P P M \\
x \\
P P M \\
x \\
P P M \\
P C I / G \\
P P M \\
\% \\
P P M \\
\% \\
P P M\end{array}$ & $\begin{array}{l}771 N G-A \\
77 \mathrm{ING}-A \\
77 \mathrm{ING}-A \\
77 \mathrm{ING}-A \\
77 \mathrm{ING}-A \\
77 \mathrm{ING}-A \\
77 \mathrm{ING}-A \\
77 \mathrm{ING}-A \\
77 \mathrm{ING}-A \\
77 \mathrm{ING}-A \\
77 \mathrm{ING}-A \\
77 \mathrm{ING}-A \\
77 \mathrm{ING}-A \\
77 \mathrm{ING}-A \\
77 \mathrm{ING}-A \\
77 \mathrm{ING}-A \\
77 \mathrm{ING}-A \\
77 \mathrm{ING}-A \\
77 \mathrm{ING}-A\end{array}$ \\
\hline \multicolumn{6}{|c|}{80.00521} \\
\hline & $\begin{array}{l}A L \\
B A \\
B 1 \\
C A \\
C D \\
C D \\
C U \\
F E \\
K \\
M G \\
M O\end{array}$ & $\begin{array}{c}7.1 \\
410 . \\
20 . \\
2.5 \\
1.2 \\
18 . \\
67 . \\
5 . \\
6500 . \\
1.3 \\
35 .\end{array}$ & & $\begin{array}{l}x \\
\text { PPM } \\
\text { PPM } \\
x \\
\text { PPM } \\
\text { PPM } \\
\text { PPM } \\
x \\
\text { PPM } \\
x \\
\text { PPM }\end{array}$ & $\begin{array}{l}77 I N G-A \\
77 I N G-A \\
77 I N G-A \\
77 I N G-A \\
77 I N G-A \\
77 I N G-A \\
77 I N G-A \\
77 I N G-A \\
77 I N G-A \\
77 I N G-A \\
77 I N G-A\end{array}$ \\
\hline
\end{tabular}


TABLE A-II (cont)

\begin{tabular}{|c|c|c|c|c|c|}
\hline $\begin{array}{l}\text { SAMPLE } \\
\text { NUMBER }\end{array}$ & $\begin{array}{l}\text { CERTIFIED } \\
\text { YALUE } \\
\text { NAME }\end{array}$ & $\begin{array}{l}\text { CERTIFIED } \\
\text { YALUE }\end{array}$ & $\begin{array}{l}\text { CERTIFIED } \\
\text { VALUE } \\
\text { UNCERTAINTY }\end{array}$ & $\begin{array}{l}\text { CERT IFIED } \\
\text { VALUE } \\
\text { UNITS }\end{array}$ & REFERENCE \\
\hline \multirow{2}{*}{80.00521} & & & & & \\
\hline & $\begin{array}{l}N A \\
P B \\
R A-226 \\
S \\
S I \\
S R \\
U \\
y\end{array}$ & $\begin{array}{c}4.2 \\
346 . \\
566 . \\
1700 . \\
28.7 \\
60 . \\
1730 . \\
720 .\end{array}$ & 12. & $\begin{array}{l}\% \\
P P M \\
P C I / G \\
P P M \\
\% \\
P P M \\
P P M \\
P P M\end{array}$ & $\begin{array}{l}771 \mathrm{NG}-A \\
771 \mathrm{NG}-A \\
77 \mathrm{NG}-A \\
771 \mathrm{NG}-A \\
77 \mathrm{ING}-A \\
77 \mathrm{ING}-A \\
77 \mathrm{ING}-A \\
77 \mathrm{ING}-A\end{array}$ \\
\hline
\end{tabular}

80.00522

$\begin{array}{lr}\text { AL } & 2.3 \\ \text { BA } & 130 . \\ \text { BI } & 40 . \\ \text { CA } & 300 . \\ \text { CD } & 270 . \\ \text { CE } & 2400 . \\ \text { CO } & 89 . \\ \text { CU } & 73 . \\ \text { DY } & 40 . \\ \text { FE } & 6.1 \\ \text { GD } & 90 .\end{array}$

KA 1150 .

MG 300 .

MO 5 .

NA 400 .

ND $\quad 750$.

PB $\quad 523$.

PR 200.

RA-226 578.

$\begin{array}{lr}S & 6.3 \\ S I & 38.5\end{array}$

SM 150 .

SR 5.

TB 20.

TH 1040.

TI 2000 .

U 1770.

14.

1

צ

$\begin{array}{ll} & 77 \text { ING-A } \\ \text { PPM } & \text { 77ING-A }\end{array}$

PPM 77ING-A

PPM 77ING-A

PPB 77ING-A

PPM 77ING-A

PPM 77ING-A

PPM 77ING-A

PPM 77ING-A

$\%$ 77ING-A

PPM 77ING-A

$\%$ 77ING-A

PPM 77ING-A

PPM 77ING-A

PPM 77ING-A

PPM 77ING-A

PPM 77ING-A

PPM 77 ING-A

PPM 77ING-A

12.

PCI/G 77 ING-A

\% 77 ING-A

$\%$ 77ING-A

PPM 77ING-A

PPM 77ING-A

PPM 77 ING-A

50. PPM 77ING-A

PPM 77ING-A

PPM 77ING-A

PPM 77ING-A

80.00523

$\begin{array}{lr}\text { AL } & 5.1 \\ \text { BA } & 160 . \\ \text { BI } & 10 . \\ \text { CA } & 600 . \\ \text { CD } & 200 . \\ \text { CE } & 98 . \\ C D & 11 . \\ \text { CU } & 74 . \\ \text { DY } & 2.1 \\ \text { FE } & 8300 . \\ \text { GD } & 3.3 \\ \text { K } & 2.5\end{array}$

$\begin{array}{ll}\% & 77 \text { ING-A } \\ \text { PPM } & \text { 77ING-A } \\ \text { PPM } & \text { 77ING-A } \\ \text { PPH } & \text { 77ING-A } \\ \text { PPB } & 77 \text { ING-A } \\ \text { PPM } & 77 \text { ING-A } \\ \text { PPM } & 77 \text { NG-A } \\ \text { PPM } & \text { 77ING-A } \\ \text { PPM } & \text { 77ING-A } \\ \text { PPM } & \text { 77ING-A } \\ \text { PPM } & \text { 77ING-A } \\ \% & \text { 77ING-A }\end{array}$


TABLE A-II (cont)

\begin{tabular}{|c|c|c|c|c|c|}
\hline $\begin{array}{l}\text { SAMPLE } \\
\text { NUMBER }\end{array}$ & $\begin{array}{l}\text { CERT IFIED } \\
\text { VALUE } \\
\text { NAME }\end{array}$ & $\begin{array}{l}\text { CERTIFIED } \\
\text { VALUE }\end{array}$ & $\begin{array}{l}\text { CERTIFIED } \\
\text { VALUE } \\
\text { UNCERTAINTY }\end{array}$ & $\begin{array}{l}\text { CERTIFIED } \\
\text { VALUE } \\
\text { UNITS }\end{array}$ & REFERENCE \\
\hline 80.00523 & $\begin{array}{l}\text { LA } \\
\text { MG } \\
\text { MO } \\
\text { NA } \\
\text { ND } \\
\text { PB } \\
\text { PR } \\
\text { RA-226 } \\
S \\
\text { SI } \\
\text { SM } \\
\text { SR } \\
\text { TH } \\
\text { TI } \\
U \\
V \\
Y \\
\text { YB }\end{array}$ & $\begin{array}{c}52 . \\
500 . \\
4 . \\
700 . \\
38 . \\
18 . \\
9.6 \\
13.2 \\
3000 . \\
37.1 \\
6.9 \\
4 . \\
83 . \\
800 . \\
41 . \\
24 . \\
17 . \\
1.3\end{array}$ & $\begin{array}{l}6 . \\
2 .\end{array}$ & $\begin{array}{l}P P M \\
P P M \\
P P M \\
P P M \\
P P M \\
P P M \\
P P M \\
P C I / G \\
P P M \\
\% \\
\text { PPM } \\
P P M \\
P P M \\
P P M \\
P P M \\
P P M \\
P P M \\
P P M\end{array}$ & 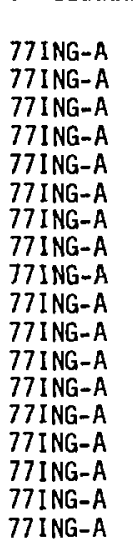 \\
\hline 80.00524 & U & 2660 . & & PPM & 67IAE-A \\
\hline 80.00525 & U & 3180. & & PPM & $67 \mathrm{IAE}-\mathrm{B}$ \\
\hline 80.00526 & U & 119. & & PPM & 74JEN-A \\
\hline $80: 00527$ & U & 1200. & & PPM & 74JEN-A \\
\hline 80.00528 & U & 4470. & & PPM & 74JEN-A \\
\hline 80.00529 & U & 339. & & PPM & 74JEN-A \\
\hline 80.00530 & U & 3540. & & PPM & 67IAE-C \\
\hline 80.00531 & $\begin{array}{l}\text { AL } \\
\text { AS } \\
B \\
B A \\
B R \\
C \\
C A \\
C D \\
C L \\
C D \\
C R \\
C S\end{array}$ & $\begin{array}{c}870 . \\
150 . \\
30 . \\
13 . \\
54 . \\
40.82 \\
1.35 \\
1.5 \\
6400 . \\
1.5 \\
4.6 \\
48 .\end{array}$ & $\begin{array}{l}50 . \\
50 .\end{array}$ & $\begin{array}{l}\text { PPM } \\
\text { PPB } \\
\text { PPM } \\
\text { PPM } \\
\text { PPM } \\
\% \\
\text { PPM } \\
\text { PPM } \\
\text { PPM } \\
\text { PPM } \\
\text { PPB }\end{array}$ & $\begin{array}{l}\text { 76NBS-A } \\
\text { 76NBS-A } \\
\text { 76NBS-A } \\
\text { 80GLA-A } \\
\text { 76NBS-A } \\
\text { 80GLA-A } \\
\text { 76NBS-A } \\
\text { 76NBS-A } \\
\text { 80GLA-A } \\
\text { 76NBS-A } \\
\text { 76NBS-A } \\
\text { 80GLA-A }\end{array}$ \\
\hline
\end{tabular}


TABLE A-II (cont)

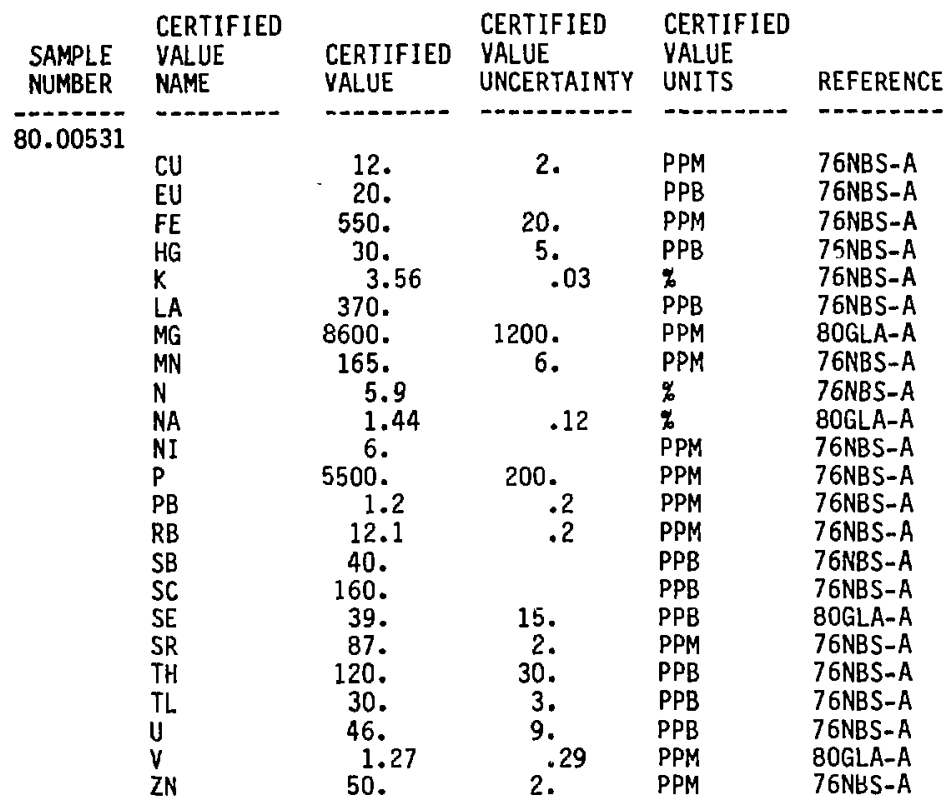

80.00532

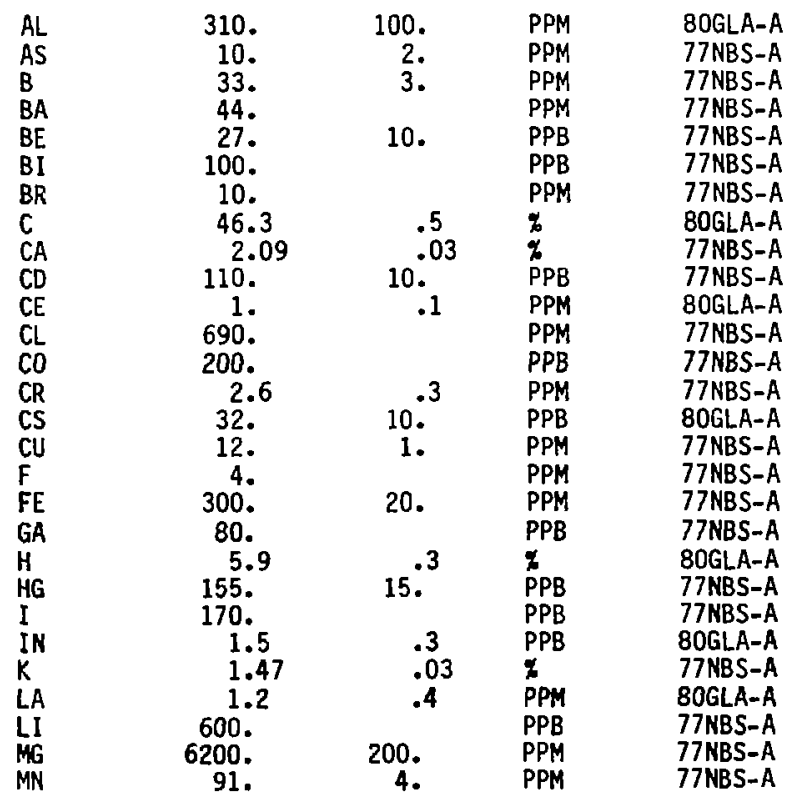


TABLE A-II (cont)

\begin{tabular}{|c|c|c|c|c|c|}
\hline $\begin{array}{l}\text { SAMPLE } \\
\text { NUMBERR }\end{array}$ & $\begin{array}{l}\text { CERTIFIED } \\
\text { VALUE } \\
\text { NAME }\end{array}$ & $\begin{array}{l}\text { CERTIF IED } \\
\text { VALUE }\end{array}$ & $\begin{array}{l}\text { CERTIF IED } \\
\text { YALUE } \\
\text { UNCERTAINTY }\end{array}$ & $\begin{array}{l}\text { CERTIF IED } \\
\text { YALUE } \\
\text { UNITS }\end{array}$ & REFERENCE \\
\hline \multirow{2}{*}{80.00532} & & & & & \\
\hline & $\begin{array}{l}M O \\
N \\
N A \\
N A \\
N I \\
P \\
P B \\
R B \\
S \\
S B \\
S E \\
S M \\
S R \\
T E \\
T H \\
U \\
Y \\
Y B \\
\text { YN }\end{array}$ & $\begin{array}{c}.3 \\
2.76 \\
82 . \\
1.3 \\
2100 . \\
45 . \\
12 . \\
1900 . \\
2.9 \\
80 . \\
110 . \\
37 . \\
10 . \\
64 . \\
28 . \\
600 . \\
27 . \\
25 .\end{array}$ & $\begin{array}{c}.1 \\
.05 \\
6 . \\
.2 \\
100 . \\
3 . \\
1 . \\
.3 \\
10 . \\
20 . \\
1 . \\
6 . \\
3 . \\
150 . \\
11 . \\
3 .\end{array}$ & $\begin{array}{l}\text { PPM } \\
\text { P } \\
\text { PPM } \\
\text { PPM } \\
\text { PPM } \\
\text { PPM } \\
\text { PPM } \\
\text { PPM } \\
\text { PPM } \\
\text { PPB } \\
\text { PPB } \\
\text { PPM } \\
\text { PPB } \\
\text { PPM } \\
\text { PPB } \\
\text { PPB } \\
\text { PPB } \\
\text { PPM }\end{array}$ & 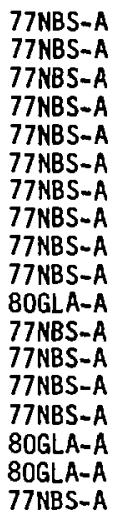 \\
\hline
\end{tabular}

80.00533

\begin{tabular}{|c|c|c|c|c|}
\hline $\begin{array}{l}\text { AL } \\
A S \\
B \\
B A \\
B R \\
C A \\
C D \\
C E \\
C L \\
C O \\
C R \\
C U \\
E U \\
F E \\
H G \\
K \\
L A \\
M G \\
M N \\
N \\
N A \\
P \\
P B \\
R B \\
\text { SC } \\
\text { SE } \\
\text { SR } \\
\text { TH } \\
T L \\
U \\
\text { ZH }\end{array}$ & $\begin{array}{c}270 . \\
30.27 \\
30 . \\
56 . \\
26 . \\
3 . \\
3 . \\
1.6 \\
1.08 \\
600 . \\
4.5 \\
11 . \\
40 . \\
690 . \\
.1 \\
4.46 \\
.9 \\
7000 . \\
238 . \\
5 . \\
530 . \\
3400 . \\
6.3 \\
16.5 \\
130 . \\
60 . \\
44.9 \\
170 . \\
50 . \\
61 . \\
62 .\end{array}$ & $\begin{array}{c}50 . \\
.05 \\
8 . \\
.03 \\
.02 \\
.5 \\
1 . \\
25 . \\
.03 \\
7 . \\
60 . \\
200 . \\
.3 \\
.1 \\
20 . \\
.3 \\
30 . \\
5 . \\
3 . \\
6 .\end{array}$ & $\begin{array}{l}P P B \\
P P M \\
P P M \\
P P M \\
P P M \\
\% \\
P P M \\
P P M \\
\% \\
P P B \\
P P M \\
P P M \\
P P B \\
P P M \\
P P M \\
\% \\
P P M \\
P P M \\
P P M \\
\% \\
P P M \\
P P M \\
P P M \\
P P M \\
P P B \\
P P B \\
P P M \\
P P B \\
P P B \\
P P B \\
P P M\end{array}$ & 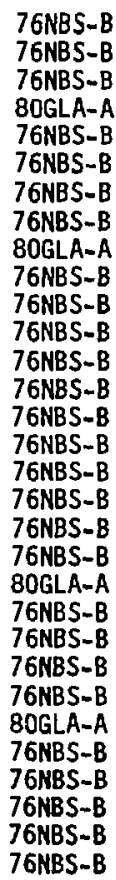 \\
\hline
\end{tabular}

80.00534 
TABLE A-II (cont)

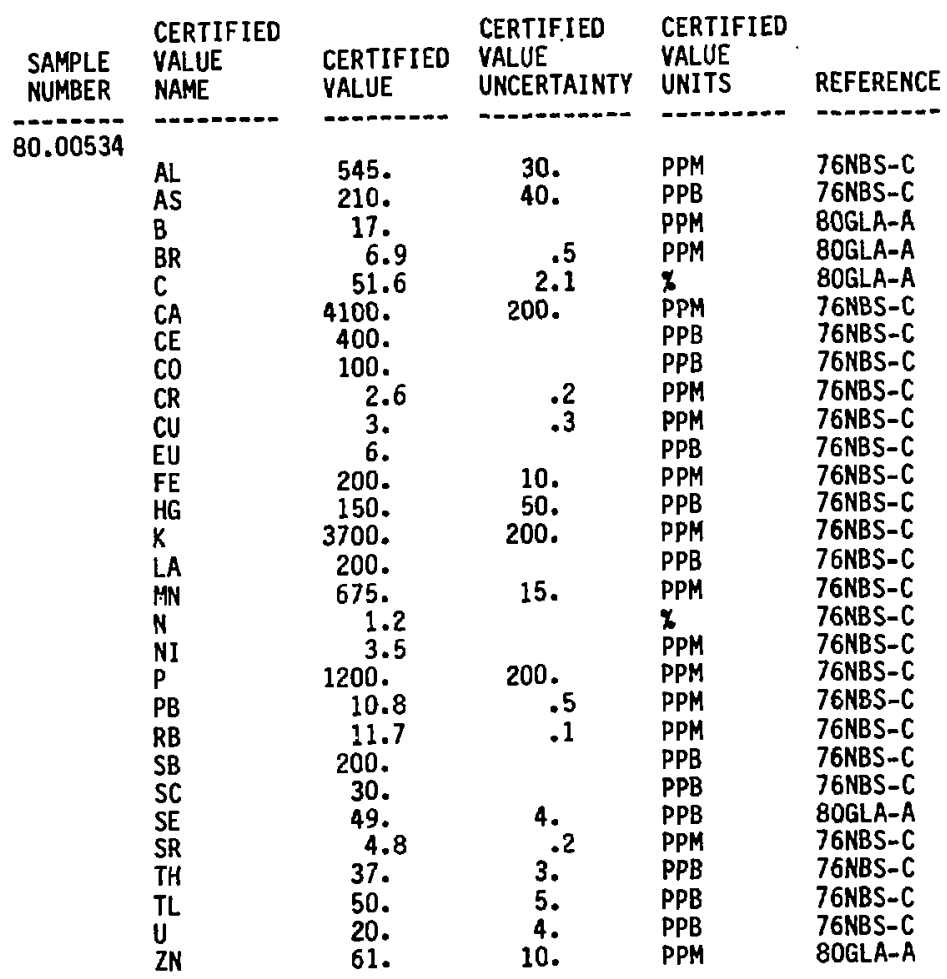

80.00535

\begin{tabular}{|c|c|c|c|c|}
\hline $\begin{array}{l}A G \\
A L \\
A S \\
B \\
B R \\
C \\
C A \\
C D \\
C L \\
C D \\
C S \\
C U \\
F E \\
H \\
H G \\
K \\
M G \\
M \\
M O \\
N \\
M A\end{array}$ & $\begin{array}{c}60 . \\
30 . \\
55 . \\
3.2 \\
8.7 \\
50.6 \\
123 . \\
370 . \\
2600 . \\
180 . \\
17 . \\
193 . \\
270 . \\
7 . \\
16 . \\
.97 \\
605 . \\
10.3 \\
3.2 \\
10.6 \\
2430 . \\
1.05\end{array}$ & $\begin{array}{c}20 . \\
.9 \\
1.7 \\
1.1 \\
40 . \\
7 . \\
10 . \\
20 . \\
.2 \\
.06 \\
1 . \\
.6 \\
130 . \\
.16\end{array}$ & 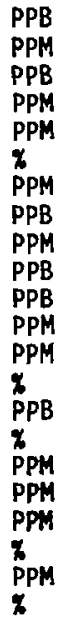 & $\begin{array}{l}\text { 72NBS-E } \\
\text { 80GLA-A } \\
72 N B S-E \\
\text { BOGLA-A } \\
\text { 80GLA-A } \\
\text { 80GLA-A } \\
\text { 72NBS-E } \\
\text { 72NBS-E } \\
\text { 72NBS-E } \\
\text { 72NBS-E } \\
\text { 80GLA-A } \\
\text { 72NBS-E } \\
\text { 72NBS-E } \\
\text { 80GLA-A } \\
\text { 72NBS-E } \\
\text { 72NBS-E } \\
\text { 72NBS-E } \\
\text { 72NBS-E } \\
\text { 72NBS-E } \\
\text { 72NBS-E } \\
\text { 72NBS-E } \\
\text { 80GLA-A }\end{array}$ \\
\hline
\end{tabular}


TABLE A-II (cont)

\begin{tabular}{|c|c|c|c|c|c|}
\hline $\begin{array}{l}\text { SAMPLE } \\
\text { NUMBER }\end{array}$ & $\begin{array}{l}\text { CERTIFIED } \\
\text { VALUE } \\
\text { NAME }\end{array}$ & $\begin{array}{l}\text { CERTIFIED } \\
\text { VALUE }\end{array}$ & $\begin{array}{l}\text { CERTIFIED } \\
\text { VALUE } \\
\text { UNCERTAINTY }\end{array}$ & $\begin{array}{l}\text { CERTIFIED } \\
\text { VALUE } \\
\text { UNITS }\end{array}$ & REFERENCE \\
\hline 635 & & & & & \\
\hline & $\begin{array}{l}P B \\
R B \\
S \\
S E \\
S R \\
T L \\
U \\
Z N\end{array}$ & $\begin{array}{c}340 . \\
18.3 \\
7100 . \\
1.1 \\
140 . \\
50 . \\
130.8 \\
130\end{array}$ & $\begin{array}{r}80 . \\
100 \\
100 \\
.1\end{array}$ & $\begin{array}{l}\text { PPB } \\
\text { PPM } \\
\text { PPM } \\
\text { PPM } \\
\text { PPB } \\
\text { PPB } \\
\text { PPB } \\
\text { PPM }\end{array}$ & $\begin{array}{l}72 N B S-E \\
72 N B S-E \\
80 G L A-A \\
72 N B S-E \\
72 N B S-E \\
72 N B S-E \\
72 N B S-E \\
72 N B S-E\end{array}$ \\
\hline
\end{tabular}

80.00536

\begin{tabular}{|c|c|c|c|c|}
\hline $\begin{array}{l}A G \\
A L \\
A S \\
B \\
B A \\
B E \\
B R \\
C \\
C A \\
C D \\
C E \\
C L \\
C O \\
C R \\
C S \\
C U \\
D Y \\
E U \\
F E \\
G A \\
G D \\
H \\
H F \\
H G \\
I \\
K \\
L A \\
L U \\
M G \\
M N \\
N \\
N A \\
N D \\
N I \\
P B \\
R B \\
S \\
S B \\
S C \\
S E \\
S I \\
S H \\
S R\end{array}$ & $\begin{array}{c}60 . \\
1.74 \\
5.9 \\
40 . \\
344 . \\
1.4 \\
18.2 \\
71 . \\
4100 . \\
190 . \\
21 . \\
880 . \\
5.7 \\
20.2 \\
1.6 \\
18.0 \\
1.2 \\
340 . \\
8700 . \\
6 . \\
2.9 \\
4.23 \\
120.9 \\
3.4 \\
2800 . \\
10.3 \\
125 . \\
1800 . \\
40 . \\
1.1 \\
376 . \\
9.2 \\
15 . \\
30 . \\
21 . \\
1.5 \\
3.6 \\
3.8 \\
2.9 \\
3.2 \\
1.6 \\
145 .\end{array}$ & $\begin{array}{c}15 . \\
.13 \\
.6 \\
9 . \\
33 . \\
.2 \\
2.1 \\
2 . \\
460 . \\
30 . \\
3 . \\
60 . \\
.5 \\
.5 \\
.5 \\
2 . \\
.3 \\
50 . \\
300 . \\
1.4 \\
1.2 \\
.18 \\
.1 \\
20 . \\
1.2 \\
120 . \\
1.2 \\
17 . \\
440 . \\
3 . \\
.2 \\
26 . \\
1.9 \\
1 . \\
9 . \\
3 . \\
.4 \\
.6 \\
.3 \\
.3 \\
.2 \\
22 .\end{array}$ & 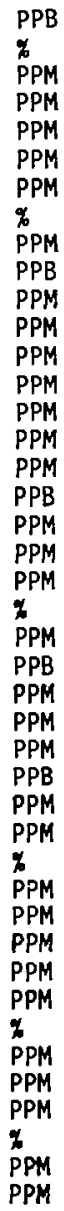 & $\begin{array}{l}\text { 80GLA-A } \\
\text { 80GLA-A } \\
\text { 74NBS-A } \\
\text { 80GLA-A } \\
\text { 80GLA-A } \\
\text { 80GLA-A } \\
\text { 80GLA-A } \\
\text { 80GLA-A } \\
\text { 80GLA-A } \\
\text { 74NBS-A } \\
\text { 80GLA-A } \\
\text { 80GLA-A } \\
\text { 80GLA-A } \\
\text { 74NBS-A } \\
\text { 80GLA-A } \\
\text { 74NBS-A } \\
\text { 80GLA-A } \\
\text { 80GLA-A } \\
\text { 74NBS-A } \\
\text { 80GLA-A } \\
\text { 80GLA-A } \\
\text { 80GLA-A } \\
\text { 80GLA-A } \\
\text { 74NBS-A } \\
\text { 80GLA-A } \\
\text { 80GLA-A } \\
\text { 80GLA-A } \\
\text { 80GLA-A } \\
\text { 80GLA-A } \\
\text { 74NBSS-A } \\
\text { 80GLA-A } \\
\text { 80GLA-A } \\
\text { 80GLA-A } \\
\text { 74NBS-A } \\
\text { 74NBS-A } \\
\text { 80GLA-A } \\
\text { 80GLA-A } \\
\text { 80GLA-A } \\
\text { 80GLA-A } \\
74 N B S-A \\
74 N B S-A \\
\text { 80GLA-A } \\
\text { 80GLA-A }\end{array}$ \\
\hline
\end{tabular}


TABLE A-II (cont)

\begin{tabular}{|c|c|c|c|c|c|}
\hline $\begin{array}{l}\text { SAMPLE } \\
\text { NUMBER }\end{array}$ & $\begin{array}{l}\text { CERTIF IED } \\
\text { VALUE } \\
\text { NAME }\end{array}$ & $\begin{array}{l}\text { CERTIFIED } \\
\text { VALUE }\end{array}$ & $\begin{array}{l}\text { CERTIF IED } \\
\text { VALUE } \\
\text { UNCERTAINTY }\end{array}$ & $\begin{array}{l}\text { CERTIFIED } \\
\text { VALUE } \\
\text { UNITS }\end{array}$ & REFERENCE \\
\hline .00536 & & & & & \\
\hline & $\begin{array}{l}\text { TA } \\
\text { TB } \\
\text { TH } \\
\text { TI } \\
\text { TL } \\
\text { U } \\
V \\
W \\
\text { YB } \\
\text { ZN } \\
\text { ZR }\end{array}$ & $\begin{array}{c}260 . \\
300 . \\
3 . \\
950 . \\
590 . \\
1.4 \\
35 . \\
740 . \\
780 . \\
37 . \\
36 .\end{array}$ & $\begin{array}{c}70 . \\
100 . \\
.7 \\
120 . \\
30 . \\
.1 \\
3 . \\
70 . \\
130 . \\
4 . \\
9 .\end{array}$ & $\begin{array}{l}\text { PPB } \\
\text { PPB } \\
\text { PPM } \\
\text { PPM } \\
\text { PPB } \\
\text { PPM } \\
\text { PPM } \\
\text { PPB } \\
\text { PPB } \\
\text { PPM } \\
\text { PPM }\end{array}$ & $\begin{array}{l}\text { 80GLA-A } \\
\text { 80GLA-A } \\
\text { 80GLA-A } \\
\text { 80GLA-A } \\
\text { 74NBS-A } \\
\text { 74NBS-A } \\
\text { 74NBS-A } \\
\text { 80GLA-A } \\
\text { 60GLA-A } \\
\text { 74NBS-A } \\
\text { 80GLA-A }\end{array}$ \\
\hline
\end{tabular}

80.00537

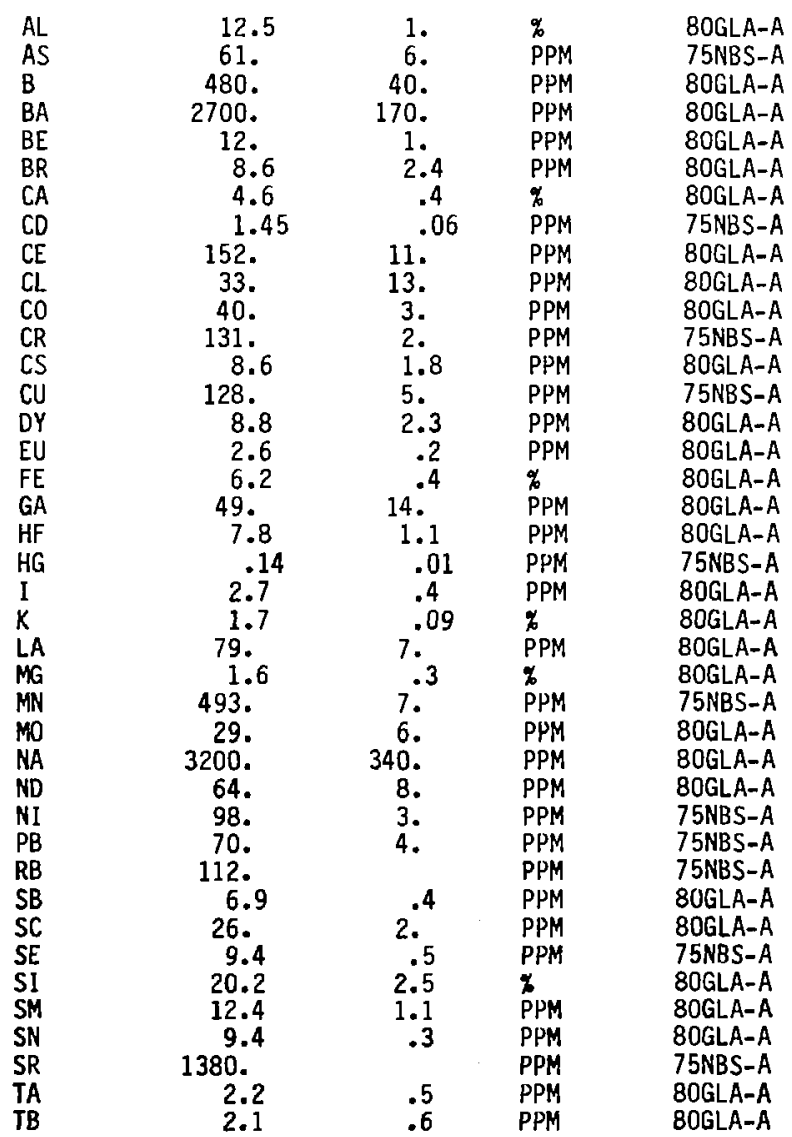


TABLE A-II (cont)

\begin{tabular}{|c|c|c|c|c|c|}
\hline $\begin{array}{l}\text { SAMPLE } \\
\text { NUMBER }\end{array}$ & $\begin{array}{l}\text { CERTIFIED } \\
\text { VALUE } \\
\text { NAME }\end{array}$ & $\begin{array}{l}\text { CERTIFIED } \\
\text { VALUE }\end{array}$ & $\begin{array}{l}\text { CERTIFIED } \\
\text { VALUE } \\
\text { UNCERTAINTY }\end{array}$ & $\begin{array}{l}\text { CERTIFIED } \\
\text { VALUE } \\
\text { UNITS }\end{array}$ & REFERENCE \\
\hline 80.00537 & - & --.-- & - & $\cdots$ & 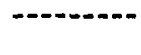 \\
\hline & $\begin{array}{l}T H \\
T I \\
T L \\
U \\
V \\
W \\
Y \\
Y B \\
Z N \\
Z R\end{array}$ & $\begin{array}{c}24 . \\
7200 . \\
4 . \\
11.6 \\
214 . \\
5.5 \\
65 . \\
6.4 \\
210 . \\
300 .\end{array}$ & $\begin{array}{l}720 . \\
.2 \\
8 . \\
2.6 \\
3 . \\
1.1 \\
20 . \\
75 .\end{array}$ & $\begin{array}{l}\text { PPM } \\
\text { PPM } \\
\text { PPM } \\
\text { PPM } \\
\text { PPM } \\
\text { PPM } \\
\text { PPM } \\
\text { PPM } \\
\text { PPM } \\
\text { PPM }\end{array}$ & $\begin{array}{l}\text { 75NBS-A } \\
\text { 80GLA-A } \\
\text { 75NBS-A } \\
\text { 75NBS-A } \\
\text { 75NBS-A } \\
\text { 80GLA-A } \\
\text { 80GLA-A } \\
\text { 80GLA-A } \\
\text { 75NBS-A } \\
\text { 80GLA-A }\end{array}$ \\
\hline 80.00538 & $H G$ & 10 & 05 & & \\
\hline 80.00539 & & & & & \\
\hline & $H G$ & 1.18 & .05 & PPB & 74NBS-B \\
\hline 80.00540 & $H G$ & 1.1 & .06 & PPB & 77NBS-B \\
\hline 80.00541 & $\begin{array}{l}U \\
U-235 \\
U-238\end{array}$ & $\begin{array}{r}84.8 \\
.72 \\
99.22\end{array}$ & & $\begin{array}{l}x \\
x \\
x\end{array}$ & $\begin{array}{l}\text { 69NBS-D } \\
\text { 76MIL-A } \\
\text { 76MIL-A }\end{array}$ \\
\hline 80.00542 & AS & 99.99 & & \% & 70NBS-C \\
\hline 80.00543 & $\begin{array}{l}K \\
N\end{array}$ & $\begin{array}{l}38.66 \\
13.85\end{array}$ & .01 & 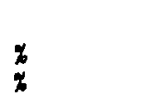 & $\begin{array}{l}\text { 74NBS-C } \\
\text { 74NBS-C }\end{array}$ \\
\hline 80.00544 & $\begin{array}{l}N \\
P\end{array}$ & $\begin{array}{l}12.15 \\
26.9\end{array}$ & .01 & $\%$ & $\begin{array}{l}\text { 74NBS-D } \\
\text { 74NBS-D }\end{array}$ \\
\hline 80.00545 & $F$ & .835 & .082 & PPM & 75NBS-C \\
\hline 80.00546 & $\begin{array}{l}\text { CA } \\
\text { F } \\
\text { SI }\end{array}$ & $\begin{array}{r}49.99 \\
47.43 \\
3100 .\end{array}$ & $\begin{array}{l}.062 \\
.062\end{array}$ & $\begin{array}{l}x \\
\text { PPM }\end{array}$ & $\begin{array}{l}\text { 71NBS-A } \\
\text { 71NBS-A } \\
\text { 71NBS-A }\end{array}$ \\
\hline 80.00547 & $\begin{array}{l}C \\
C A \\
C D \\
F \\
M G \\
M N \\
N A \\
P\end{array}$ & $\begin{array}{c}7600 . \\
35.32 \\
20 . \\
3.84 \\
1700 . \\
250 . \\
2600 . \\
15.1\end{array}$ & & $\begin{array}{l}\text { PPM } \\
\% \\
\text { PPM } \\
\% \\
\text { PPM } \\
\text { PPM } \\
\text { PPM } \\
x\end{array}$ & $\begin{array}{l}\text { 72NBS-F } \\
\text { 72NBS-F } \\
\text { 72NBS-F } \\
\text { 72NBS-F } \\
\text { 72NBS-F } \\
\text { 72NBS-F } \\
\text { 72NBS-F } \\
\text { 72NBS-F }\end{array}$ \\
\hline
\end{tabular}


TABLE A-II (cont)

\begin{tabular}{|c|c|c|c|c|c|}
\hline $\begin{array}{l}\text { SAMPLE } \\
\text { NUMBER }\end{array}$ & $\begin{array}{l}\text { CERTIFIED } \\
\text { VALUE } \\
\text { NAME }\end{array}$ & $\begin{array}{l}\text { CERTIFIED } \\
\text { VALUE }\end{array}$ & $\begin{array}{l}\text { CERT IFIED } \\
\text { VALUE } \\
\text { UNCERTAINTY }\end{array}$ & $\begin{array}{l}\text { CERTIFIED } \\
\text { VALUE } \\
\text { UNITS }\end{array}$ & REFERENCE \\
\hline \multirow{2}{*}{80.00547} & - & & - & & \\
\hline & $\begin{array}{l}\text { PB } \\
\text { SI } \\
\text { TI }\end{array}$ & $\begin{array}{l}900 . \\
2.19 \\
900 .\end{array}$ & & $\begin{array}{l}\text { PPM } \\
\% \\
\text { PPM }\end{array}$ & $\begin{array}{l}72 \mathrm{NBS}-\mathrm{F} \\
72 \mathrm{NBS}-\mathrm{F} \\
72 \mathrm{NBS}-\mathrm{F}\end{array}$ \\
\hline
\end{tabular}

80.00548

$\begin{array}{lc}\text { AL } & 3.18 \\ \text { AS } & 690 . \\ \text { CA } & 7.49 \\ \text { CL } & 140.9 \\ \text { F } & 5.72 \\ \text { FE } & 570 . \\ \text { K } & 2.7 \\ \text { NA } & 6.29 \\ \text { P } & 100 . \\ \text { SI } & 31.54 \\ \text { TI } & 110 . \\ \text { ZN } & 600 . \\ \text { ZR } & 70 .\end{array}$

$\begin{array}{ll}\% & \text { 31NBS-A } \\ \text { PPM } & \text { 31NBS-A } \\ \% & \text { 31NBS-A } \\ \text { PPM } & \text { 31NBS-A } \\ \% & \text { 31NBS-A } \\ \text { PPM } & \text { 31NBS-A } \\ \% & \text { 31NBS-A } \\ \% & \text { 31NBS-A } \\ \% & \text { 31NBS-A } \\ \text { PPM } & \text { 31NBS-A } \\ \% & \text { 31NBS-A } \\ \text { PPM } & \text { 31NBS-A } \\ \text { PPM } & \text { 31NBS-A }\end{array}$

80.00549

$\begin{array}{lr}\text { AL } & 2 . \\ \text { CA } & 46.1 \\ \text { F } & 800 . \\ \text { FE } & 2.9\end{array}$

$K \quad 1400$.

MG $\quad 6300$.

MN 300 .

NA 4800 .

$P \quad 1000$.

S $\quad 8800$.

SI $\quad 10.2$

SR 2600 .

TI 1400 .

\begin{tabular}{|c|c|}
\hline $\begin{array}{l}\% \\
\% \\
\text { PPM } \\
\% \\
\text { PPM } \\
\text { PPM } \\
\text { PPM } \\
\text { PPM } \\
\text { PPM } \\
\text { PPM } \\
\% \\
\text { PPM } \\
\text { PPM }\end{array}$ & $\begin{array}{l}75 \mathrm{NBS}- \\
75 \mathrm{NBS}- \\
75 \mathrm{NBS}- \\
75 \mathrm{NBS}- \\
75 \mathrm{NBS}- \\
75 \mathrm{NBS}- \\
75 \mathrm{NBS}- \\
75 \mathrm{NBS}- \\
75 \mathrm{NBS}- \\
75 \mathrm{NBS}- \\
75 \mathrm{NBS}- \\
75 \mathrm{NBS}- \\
75 \mathrm{NBS}-\end{array}$ \\
\hline
\end{tabular}

80.00550

$\begin{array}{lc}\text { AL } & 3.3 \\ C A & 42.8 \\ F & 300 . \\ \text { FE } & 1.83 \\ \text { K } & 3700 . \\ \text { MG } & 7500 . \\ \text { MN } & 600 . \\ \text { NA } & 500 . \\ \text { P } & 740 . \\ \text { PB } & 426 . \\ \text { S } & 2.83 \\ \text { SI } & 8.6 \\ \text { SR } & 1800 . \\ \text { TI } & 1900 . \\ \text { ZN } & 80 .\end{array}$

$\begin{array}{ll}\% & 75 N B S-D \\ \% & 75 N B S-D \\ \text { PPM } & 75 N B S-D \\ \% & 75 N B S-D \\ \text { PPM } & 75 N B S-D \\ \text { PPM } & 75 N B S-D \\ \text { PPM } & 75 N B S-D \\ \text { PPM } & 75 N B S-D \\ \text { PPM } & 75 N B S-D \\ \text { PPH } & 75 N B S-D \\ \% & 75 N B S-D \\ \% & 75 N B S-D \\ \text { PPM } & 75 N B S-D \\ \text { PPM } & 75 N B S-D \\ \text { PPM } & 75 N B S-D\end{array}$

80.00552

CA

74NBS-E 
TABLE A-II (cont)

\begin{tabular}{|c|c|c|c|c|c|}
\hline $\begin{array}{l}\text { SAMPLE } \\
\text { NUMBER }\end{array}$ & $\begin{array}{l}\text { CERTIFIED } \\
\text { VALUE } \\
\text { NAME }\end{array}$ & $\begin{array}{l}\text { CERTIFIED } \\
\text { VALUE }\end{array}$ & $\begin{array}{l}\text { CERTIFIED } \\
\text { VALUE } \\
\text { UNCERTAINTY }\end{array}$ & $\begin{array}{l}\text { CERTIFIED } \\
\text { VALUE } \\
\text { UNITS }\end{array}$ & REFERENCE \\
\hline \multirow{2}{*}{80.00552} & $----n-\infty$ & $--m-n-n$ & 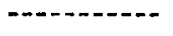 & ----- & - n- \\
\hline & $\begin{array}{l}C D \\
C O \\
C U \\
F E \\
M G \\
N I \\
P B \\
S \\
S I \\
Z N\end{array}$ & $\begin{array}{c}7800 . \\
1100 . \\
3100 . \\
2.08 \\
.45 \\
700 . \\
28000 . \\
30.6 \\
.72 \\
57.3\end{array}$ & & $\begin{array}{l}\text { PPM } \\
\text { PPM } \\
\text { PPM } \\
\% \\
\% \\
\text { PPM } \\
\text { PPM } \\
\% \\
\% \\
\%\end{array}$ & $\begin{array}{l}74 N B S-E \\
74 N B S-E \\
74 N B S-E \\
74 N B S-E \\
74 N B S-E \\
74 N B S-E \\
74 N B S-E \\
74 N B S-E \\
74 N B S-E \\
74 N B S-E\end{array}$ \\
\hline \multirow[t]{2}{*}{80.00553} & & & & & \\
\hline & $\begin{array}{l}A S \\
B I \\
C U \\
N I \\
P B \\
S B \\
S N\end{array}$ & $\begin{array}{l}570 . \\
520 . \\
540 . \\
30 . \\
84 . \\
10.26 \\
5.84\end{array}$ & & $\begin{array}{l}\text { PPM } \\
\text { PPM } \\
\text { PPM } \\
\text { PPM } \\
\% \\
\% \\
\%\end{array}$ & $\begin{array}{l}\text { 70NBS-D } \\
\text { 70NBS-D } \\
\text { 70NBS-D } \\
\text { 70NBS-D } \\
\text { 70NBS-D } \\
\text { 70NBS-D } \\
70 N B S-D\end{array}$ \\
\hline
\end{tabular}

80.00554

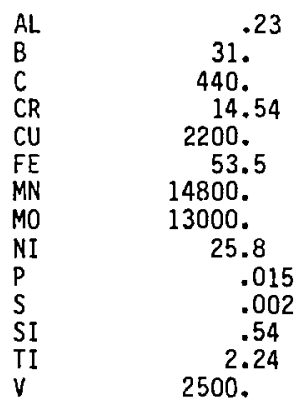

$\begin{array}{ll}\% & 66 \text { NBS-K } \\ \text { PPM } & 66 \text { NBS-K } \\ \text { PPM } & 66 N B S-K \\ \% & 66 N B S-K \\ \text { PPM } & 66 N B S-K \\ \% & 66 N B S-K \\ \text { PPM } & 66 N B S-K \\ \text { PPM } & 66 N B S-K \\ \% & 66 N B S-K \\ \% & 66 N B S-K \\ \% & 66 N B S-K \\ \% & 66 N B S-K \\ \% & 66 N B S-K \\ \text { PPM } & 66 N B S-K\end{array}$

80.00555

$\begin{array}{lc}\text { CR } & 290 . \\ \text { CU } & 79200 . \\ \text { FE } & .9 \\ \text { MG } & .002 \\ \text { MN } & 410 . \\ \text { NI } & 300 . \\ \text { PB } & 310 . \\ \text { SI } & .68 \\ \text { TI } & .35 \\ \text { ZN } & 1.5\end{array}$

$\begin{array}{ll}\text { PPM } & \text { ??NBS-A } \\ \text { PPM } & \text { ??NBS-A } \\ \% & \text { ??NBS-A } \\ \% & \text { ??NBS-A } \\ \text { PPM } & \text { ??NBS-A } \\ \text { PPM } & \text { ??NBS-A } \\ \text { PPM } & \text { ??NBS-A } \\ \% & \text { ??NBS-A } \\ \% & \text { ??NBS-A } \\ \% & \text { ??NBS-A }\end{array}$

80.00556

$\begin{array}{lc}C & 1200 . \\ \text { CR } & 12.89 \\ \text { CU } & 1180 . \\ \text { MN } & 10300 .\end{array}$

$\begin{array}{ll}\text { PPM } & \text { 56NBS-A } \\ 7 & \text { 56NBS-A } \\ \text { PPM } & \text { 56NBS-A } \\ \text { PPM } & 56 \text { NBS-A }\end{array}$


TABLE A-II (cont)

\begin{tabular}{|c|c|c|c|c|c|}
\hline $\begin{array}{l}\text { SAMPLLE } \\
\text { NIMMBER }\end{array}$ & $\begin{array}{l}\text { CERTIFIED } \\
\text { VALUE } \\
\text { NAME }\end{array}$ & $\begin{array}{l}\text { CERTIFIEU } \\
\text { VALUE }\end{array}$ & $\begin{array}{l}\text { CERTIFIEO } \\
\text { VALUE } \\
\text { UNCERTAINTY }\end{array}$ & $\begin{array}{l}\text { CERIIFIED } \\
\text { VALUE } \\
\text { UNITS }\end{array}$ & REFERENCE \\
\hline \multirow{2}{*}{80.00556} & -............. & - non-con & - n.... & $\cdots$ & \\
\hline & $\begin{array}{l}M O \\
N \\
N I \\
P \\
S \\
S I \\
V\end{array}$ & $\begin{aligned} & 2940 . .032 \\
& 2410 . .026 \\
& .026 \\
& .329 \\
& .412 \\
& 260 .\end{aligned}$ & & $\begin{array}{l}P P M \\
q \\
P P M \\
q \\
q \\
\% \\
\text { PPM }\end{array}$ & $\begin{array}{l}\text { 56NBS-A } \\
\text { 56NBS-A } \\
56 N B S-A \\
\text { 56NBS-A } \\
\text { 56NBS-A } \\
\text { 56NBS-A } \\
\text { 56NBS-A }\end{array}$ \\
\hline \multirow{2}{*}{80.00557} & & & & & \\
\hline & $\begin{array}{l}C \\
C O \\
C R \\
C U \\
F E \\
M N \\
M 0 \\
N B \\
N I \\
P \\
P B \\
S \\
S I \\
T A \\
T I \\
V\end{array}$ & $\begin{array}{c}3700 . \\
41.2 \\
20.33 \\
350 . \\
3.43 \\
15000 . \\
39500 . \\
2.95 \\
20.25 \\
.008 \\
135 . \\
.005 \\
.8 \\
9500 . \\
.06 \\
300 .\end{array}$ & & $\begin{array}{l}P P M \\
\% \\
\% \\
\text { PPM } \\
\% \\
P P M \\
P P M \\
\% \\
\% \\
\% \\
P P M \\
\% \\
\% \\
P P M \\
\% \\
P P M\end{array}$ & $\begin{array}{l}56 N B S-B \\
56 N B S-B \\
56 N B S-B \\
56 N B S-B \\
56 N B S-B \\
56 N B S-B \\
56 N B S-B \\
56 N B S-B \\
56 N B S-B \\
56 N B S-B \\
56 N B S-B \\
56 N B S-B \\
56 N B S-B \\
56 N B S-B \\
56 N B S-B \\
56 N B S-B\end{array}$ \\
\hline
\end{tabular}

80.00558

$\begin{array}{lc}\text { AL } & 5.15 \\ \text { CD } & 155 . \\ \text { CR } & 8 . \\ \text { CU } & 15000 . \\ \text { FE } & .017 \\ \text { MG } & .094 \\ \text { MN } & 17 . \\ \text { NI } & 75 . \\ \text { SI } & .078 \\ \text { SN } & .012 \\ \text { W } & 3.95\end{array}$

$\begin{array}{ll}\% & \text { 64NBS-A } \\ \text { PPM } & \text { 64NBS-A } \\ \text { PPM } & \text { 64NBS-A } \\ \text { PPM } & \text { 64NBS-A } \\ \% & \text { 64NBS-A } \\ \% & \text { 64NBS-A } \\ \text { PPM } & \text { 64NBS-A } \\ \text { PPM } & \text { 64NBS-A } \\ \% & \text { 64NBS-A } \\ \% & \text { 64NBS-A } \\ \% & 64 N B S-A\end{array}$

80.00559

$\begin{array}{lc}\text { AL } & .41 \\ B & 59 . \\ C & 450 . \\ C O & 700 . \\ C R & 12.9 \\ \text { FE } & 41.9 \\ \text { MN } & 470 . \\ \text { MO } & 32500 . \\ \text { NI } & 32.6 \\ P & .008 \\ S & .002 \\ \text { SI } & .13 \\ \text { TI } & 2.32\end{array}$

$\begin{array}{ll}\% & \text { 74NBS-F } \\ \text { PPM } & \text { 74NBS-F } \\ \text { PPM } & 74 N B S-F \\ \text { PPM } & 74 N B S-F \\ \% & 74 N B S-F \\ \% & 74 N B S-F \\ \text { PPM } & 74 N B S-F \\ \text { PPM } & 74 N B S-F \\ \% & 74 N B S-F \\ \% & 74 N B S-F \\ \% & 74 N B S-F \\ \% & 74 N B S-F \\ \% & 74 N B S-F\end{array}$


TABLE A-II (cont)

\begin{tabular}{|c|c|c|c|c|c|}
\hline $\begin{array}{l}\text { SAMPLE } \\
\text { NUMBER }\end{array}$ & $\begin{array}{l}\text { CERTIFIED } \\
\text { VALUE } \\
\text { NAME }\end{array}$ & $\begin{array}{l}\text { CERTIFIER } \\
\text { VALUE }\end{array}$ & $\begin{array}{l}\text { CERTIFIED } \\
\text { VALUE } \\
\text { UNCERTAINTY }\end{array}$ & $\begin{array}{l}\text { CERTIFIED } \\
\text { VALUE } \\
\text { UNITS }\end{array}$ & REFERENCE \\
\hline 80.00559 & ...- & $\cdots$ & & & \\
\hline & $\begin{array}{l}W \\
Z R\end{array}$ & $\begin{array}{r}6.08 \\
.15\end{array}$ & & $x$ & $\begin{array}{l}\text { 74NBS-F } \\
\text { 74NBS-F }\end{array}$ \\
\hline 80.00561 & & & & & \\
\hline & $\begin{array}{l}N \\
0\end{array}$ & $1300^{.0945}$ & & PPM & $\begin{array}{l}\text { 69NBS-E } \\
\text { 69NBS-E }\end{array}$ \\
\hline 80.00562 & 0 & 60. & & PPM & 69NBS-F \\
\hline 80.00563 & $\begin{array}{l}\text { CR } \\
\text { FE } \\
M D\end{array}$ & $\begin{array}{l}10300 . \\
1.36 \\
36100 .\end{array}$ & & $\begin{array}{l}\text { PPM } \\
\% \\
\text { PPM }\end{array}$ & $\begin{array}{l}\text { 60NBS-A } \\
\text { 60NBS-A } \\
\text { 6ONBS-A }\end{array}$ \\
\hline
\end{tabular}

80.00564

\begin{tabular}{ll} 
AL & \multicolumn{1}{c}{.0028} \\
$C$ & 270. \\
CR & 220. \\
$C U$ & 560. \\
MN & 940. \\
MO & 140. \\
NI & 500. \\
P & .006 \\
S & .026 \\
SI & 3.19 \\
SN & .004
\end{tabular}

$\begin{array}{ll}\% & \text { 72NBS-G } \\ \text { PPM } & \text { 72NBS-G } \\ \text { PPM } & \text { 72NBS-G } \\ \text { PPM } & \text { 72NBS-G } \\ \text { PPM } & \text { 72NBS-G } \\ \text { PPM } & \text { 72NBS-G } \\ \text { PPM } & \text { 72NBS-G } \\ \% & \text { 72NBS-G } \\ \% & \text { 72NBS-G } \\ \% & \text { 72NBS-G } \\ \% & \text { 72NBS-G }\end{array}$

80.00565

$\begin{array}{lc}\text { AG } & 11 . \\ \text { AL } & .095 \\ \text { AS } & 920 . \\ \text { B } & 25 . \\ \text { BI } & 20 . \\ C & 1600 . \\ \text { CA } & .0001 \\ \text { CE } & 15 . \\ C 0 & 3000 . \\ \text { CR } & 3000 . \\ \text { CU } & 5000 . \\ \text { FE } & 95.3 \\ \text { HF } & 3 . \\ \text { LA } & 4 . \\ \text { MG } & .0006 \\ \text { MN } & 10400 . \\ \text { MO } & 680 . \\ \text { N } & .004 \\ \text { NB } & .29 \\ \text { ND } & 6.4 \\ \text { NI } & 5900 . \\ 0 & 10.7 \\ \text { P } & .042 \\ \text { PB } & 4.3\end{array}$

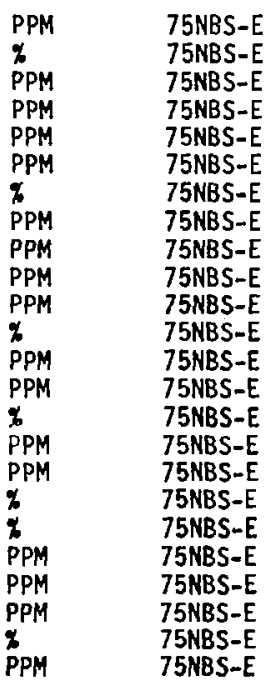


TABLE A-II (cont)

\begin{tabular}{|c|c|c|c|c|c|}
\hline $\begin{array}{l}\text { SAMPLE } \\
\text { NUMBER }\end{array}$ & $\begin{array}{l}\text { CERTIFIED } \\
\text { VALUE } \\
\text { NAME }\end{array}$ & $\begin{array}{l}\text { CERTIFIED } \\
\text { VALUE }\end{array}$ & $\begin{array}{l}\text { CERTIFIED } \\
\text { VALUE } \\
\text { UNCERTAINTY }\end{array}$ & $\begin{array}{l}\text { CERTIFIED } \\
\text { VALUE } \\
\text { UNITS }\end{array}$ & REFERENCE \\
\hline \multirow{2}{*}{80.00565} & 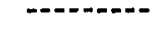 & - n-non & - & $\cdots$ & - n-..- \\
\hline & $\begin{array}{l}P R \\
S \\
S B \\
S E \\
S I \\
S N \\
T A \\
T E \\
T I \\
V \\
W \\
Z N \\
Z R\end{array}$ & $\begin{array}{c}1.2 \\
.038 \\
.012 \\
12 . \\
.39 \\
.016 \\
2000 . \\
11 . \\
410.084 \\
.2 \\
.0005 \\
.19\end{array}$ & & $\begin{array}{l}\text { PPM } \\
\% \\
\% \\
\text { PPM } \\
\% \\
\% \\
\text { PPM } \\
\text { PPM } \\
\% \\
\text { PPM } \\
\% \\
\% \\
\%\end{array}$ & $\begin{array}{l}\text { 75NBS-E } \\
75 \mathrm{NBS}-\mathrm{E} \\
75 \mathrm{NBS}-\mathrm{E} \\
75 \mathrm{NBS}-\mathrm{E} \\
75 \mathrm{NBS}-\mathrm{E} \\
75 \mathrm{NBS}-\mathrm{E} \\
\text { 75NBS-E } \\
\text { 75NBS-E } \\
\text { 75NBS-E } \\
\text { 75NBS-E } \\
\text { 75NBS-E } \\
\text { 75NBS-E } \\
\text { 75NBS-E }\end{array}$ \\
\hline \multirow{2}{*}{80.00566} & & & & & \\
\hline & $\begin{array}{l}A G \\
A L \\
A S \\
A U \\
A U \\
B \\
B I \\
C \\
C A \\
C E \\
C 0 \\
C R \\
C U \\
F E \\
H F \\
L A \\
M G \\
M N \\
N O \\
N \\
N B \\
N D \\
N I \\
0 \\
P \\
P B \\
P R \\
S \\
S B \\
S E \\
S I \\
S N \\
T A \\
T E \\
T I \\
V \\
W \\
Z N \\
Z R\end{array}$ & $\begin{array}{c}.2 \\
520.008 \\
1 . \\
110 . \\
9 . \\
8700 . \\
.4 \\
2.2 \\
1500 . \\
650 . \\
2490 . \\
96.7 \\
13 . \\
.7 \\
.0002 \\
2550 . \\
4900 . \\
.0032 \\
.16 \\
.7 \\
1420 . \\
10 . \\
.018 \\
240 . \\
.3 \\
.028 \\
.034 \\
2.1 \\
.067 \\
.008 \\
1100 . \\
1.8 \\
.24 \\
1050 . \\
.102 \\
.001 \\
.068\end{array}$ & $\because$ & 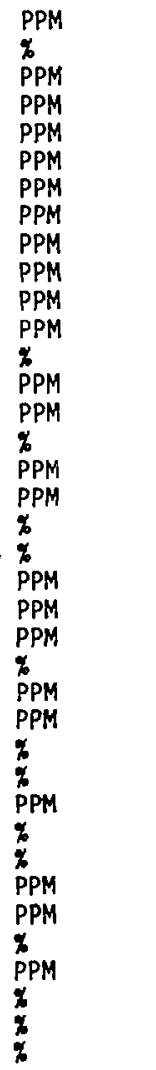 & 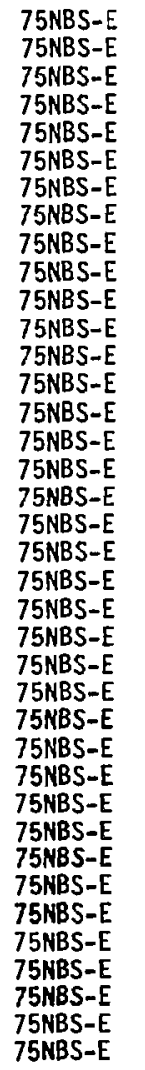 \\
\hline
\end{tabular}


TABLE A-II (cont)

\begin{tabular}{|c|c|c|c|c|}
\hline $\begin{array}{l}\text { SAMPLE } \\
\text { NUMBBER }\end{array}$ & $\begin{array}{l}\text { CERTIFIED } \\
\text { VALUE } \\
\text { NAME }\end{array}$ & $\begin{array}{l}\text { CERTIFIED } \\
\text { VALUE }\end{array}$ & $\begin{array}{l}\text { CERTIFIED } \\
\text { VALUE } \\
\text { UNCERTAINTY }\end{array}$ & $\begin{array}{l}\text { CERTIF IED } \\
\text { VALUE } \\
\text { UNITS }\end{array}$ \\
\hline & & & & \\
\hline
\end{tabular}

80.00567

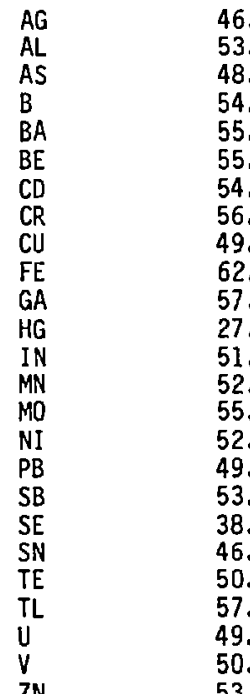

46.

5. PPM 76AND-A

53.

54.

55.

55.

54.

56.

49.

62.

57.

27.

51.

52.

52.

49.

53.

38.

56.

57.

59.

53.

8.

PPM 76AND-A

76AND-A

PPM 76AND-A
PPM 76AND-A

PPM 76AND-A

76AND-A
PPM 76AND-A

9. PPM 76AND-A

5. PPM 76AND-A

11. PPM 76AND-A

PPM 76AND-A

6.

76AND-A

7. PPM

7. $\quad P P M$

76AND-A

76AND-A

76AND-A

4. PPM

6. PPM

4. PPM

76AND-A

76AND-A

76AND-A

76AND-A

76AND-A

$\begin{array}{ll}\text { PPM } & \text { 76AND-A } \\ \text { PPM } & \text { 76AND-A } \\ \text { PPM } & \text { 76AND-A }\end{array}$

$\begin{array}{ll}\text { PPM } & \text { 76AND-A } \\ \text { PPM } & \text { 76AND-A } \\ \text { PPM } & \text { 76AND-A }\end{array}$

4.

76AND-A

80.00570

$\begin{array}{lc}\text { AG } & .11 \\ \text { AL } & 9.13 \\ \text { AS } & .8 \\ \text { AU } & .6 \\ \text { B } & 5 . \\ \text { BA } & 1208 . \\ \text { BE } & 3 . \\ \text { BI } & .057 \\ \text { BR } & .5 \\ \text { CA } & 3.48 \\ \text { CD } & .09 \\ \text { CE } & 63 . \\ \text { CL } & 110 . \\ \text { CO } & 14.1 \\ \text { CR } & 12.2 \\ \text { CS } & 1.4 \\ C U & 59.7 \\ \text { DY } & 3.5 \\ \text { ER } & 1.2 \\ \text { EU } & 1.7 \\ \text { F } & 435 . \\ \text { FE } & 4.73 \\ \text { GA } & 20.5 \\ \text { GD } & 5.5\end{array}$

$\begin{array}{ll}\text { PPM } & \text { 73FLA-A } \\ \% & \text { 73FLA-A } \\ \text { PPM } & \text { 73FLA-A } \\ \text { PPB } & \text { 73FLA-A } \\ \text { PPM } & \text { 73FLA-A } \\ \text { PPM } & \text { 73FLA-A } \\ \text { PPM } & \text { 73FLA-A } \\ \text { PPM } & \text { 73FLA-A } \\ \text { PPM } & \text { 73FLA-A } \\ \% & \text { 73FLA-A } \\ \text { PPM } & \text { 73FLA-A } \\ \text { PPM } & \text { 73FLA-A } \\ \text { PPM } & \text { 73FLA-A } \\ \text { PPM } & \text { 73FLA-A } \\ \text { PPM } & \text { 73FLA-A } \\ \text { PPM } & \text { 73FLA-A } \\ \text { PPM } & \text { 73FLA-A } \\ \text { PPM } & \text { 73FLA-A } \\ \text { PPM } & \text { 73FLA-A } \\ \text { PPM } & \text { 73FLA-A } \\ \text { PPM } & \text { 73FLA-A } \\ \% & \text { 73FLA-A } \\ \text { PPM } & \text { 73FLA-A } \\ \text { PPM } & \text { 73FLA-A }\end{array}$


TABLE A-II (cont)

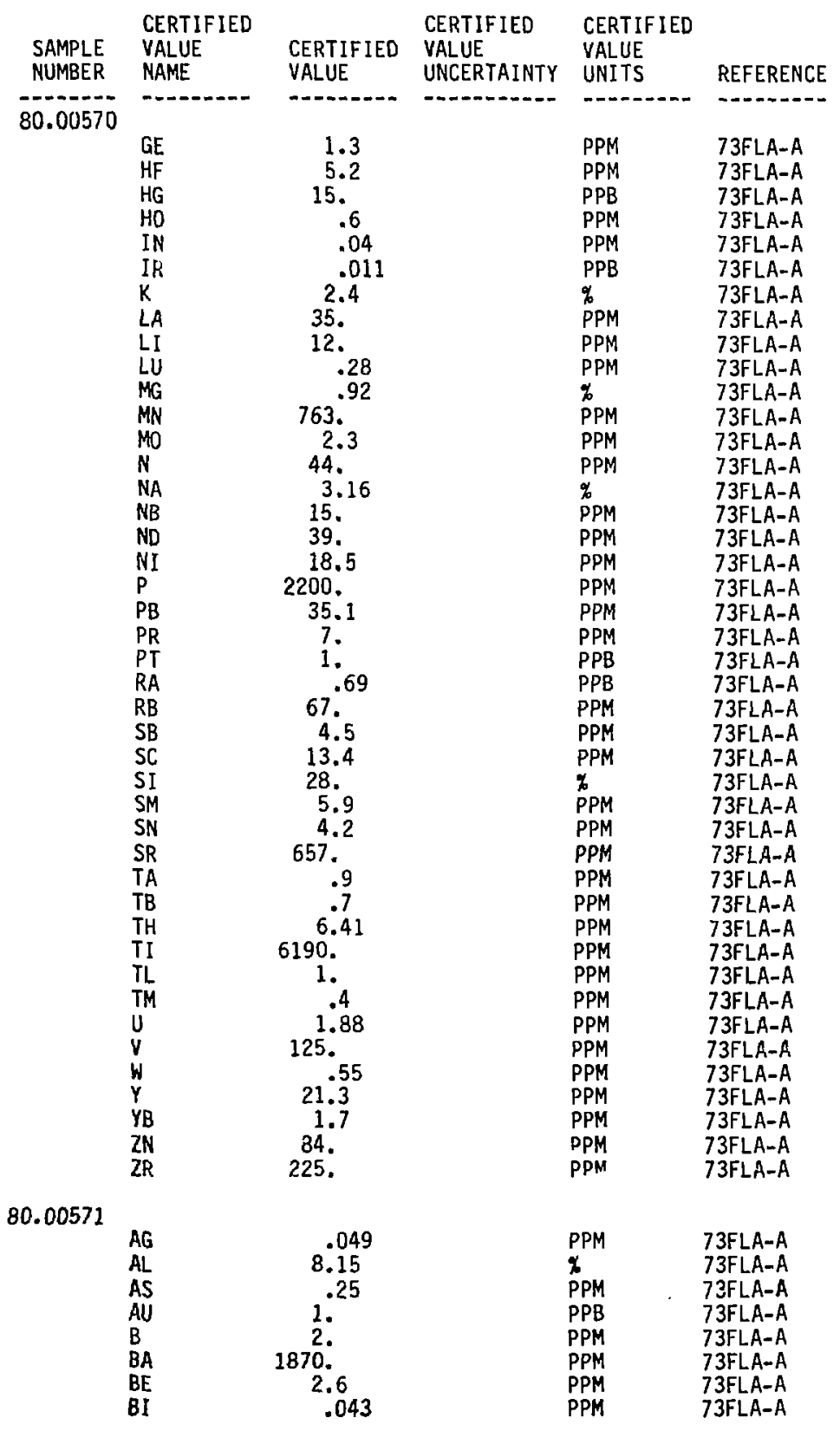


TABLE A-II (cont)

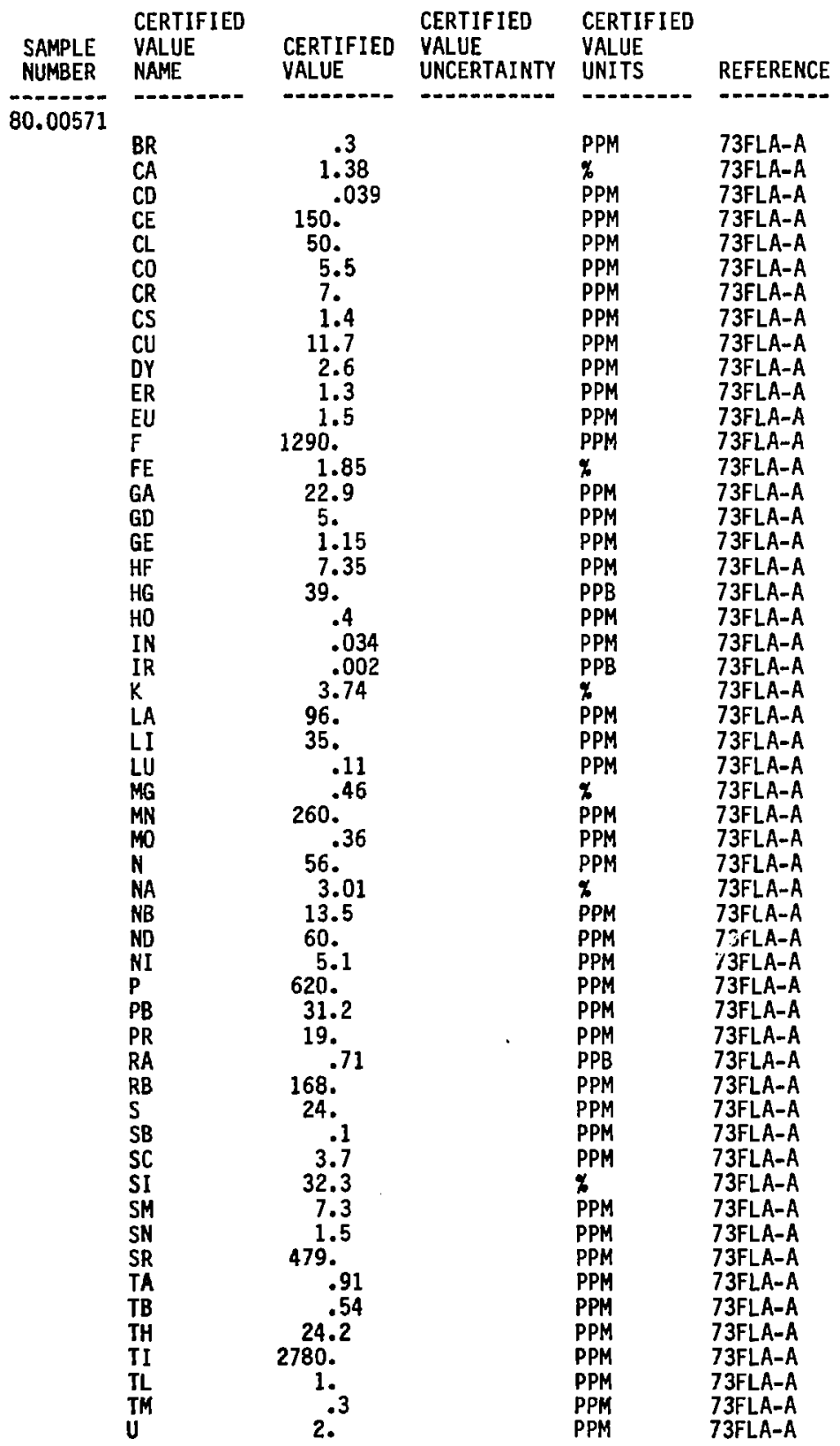


TABLE A-II (cont)

\begin{tabular}{|c|c|c|c|c|c|}
\hline $\begin{array}{l}\text { SAMPLE } \\
\text { NUMBER }\end{array}$ & $\begin{array}{l}\text { CERTIFIED } \\
\text { VALUE } \\
\text { NAME }\end{array}$ & $\begin{array}{l}\text { CERTIFIED } \\
\text { VALUE }\end{array}$ & $\begin{array}{l}\text { CERTIFIED } \\
\text { VALUE } \\
\text { UNCERTAINTY }\end{array}$ & $\begin{array}{l}\text { CERTIFIED } \\
\text { VALUE } \\
\text { UNITS }\end{array}$ & REFERENCE \\
\hline \multirow{2}{*}{80.00571} & & & & & \\
\hline & $\begin{array}{l}Y \\
W \\
Y \\
Y B \\
Z N \\
Z R\end{array}$ & $\begin{array}{c}35.4 \\
.1 \\
12 . \\
.88 \\
85 . \\
300 .\end{array}$ & & $\begin{array}{l}\text { PPM } \\
\text { PPM } \\
\text { PPM } \\
\text { PPM } \\
\text { PPM } \\
\text { PPM }\end{array}$ & $\begin{array}{l}\text { 73FLA-A } \\
\text { 73FLA-A } \\
\text { 73FLA-A } \\
\text { 73FLA-A } \\
\text { 73FLA-A } \\
\text { 73FLA-A }\end{array}$ \\
\hline
\end{tabular}

80.00572

\begin{tabular}{|c|c|c|c|}
\hline $\begin{array}{l}A G \\
A L \\
A S \\
A U \\
B \\
B A \\
B E \\
B I \\
B R \\
C \\
C A \\
C D \\
C E \\
C L \\
C D \\
C R \\
C S \\
C U \\
D Y \\
E R \\
E U \\
F \\
F E \\
G A \\
G D \\
G E \\
H F \\
H G \\
H O \\
I N \\
I R \\
K \\
L A \\
L I \\
L U \\
M G \\
W N \\
M O \\
N \\
N A \\
N B \\
N D \\
N I \\
O S \\
P\end{array}$ & $\begin{array}{c}.036 \\
7.26 \\
700 . \\
.95 \\
5 . \\
675 . \\
1.7 \\
.05 \\
.15 \\
330 . \\
4.91 \\
.12 \\
53.9 \\
50 . \\
38 . \\
17.6 \\
.95 \\
18.4 \\
6.3 \\
3.59 \\
1.94 \\
470 . \\
9.37 \\
20 . \\
6.6 \\
1.54 \\
4.7 \\
10.7 \\
1.2 \\
.095 \\
.004 \\
4.59 \\
26 . \\
12.8 \\
.55 \\
2.08 \\
1406 . \\
1.1 \\
30 . \\
2.08 \\
13.5 \\
29.9 \\
15.8 \\
.1 \\
620 .\end{array}$ & 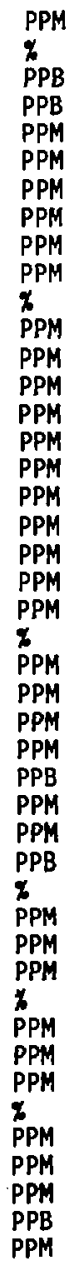 & $\begin{array}{l}\text { 73FLA-A } \\
\text { 73FLA-A } \\
\text { 73FLA-A } \\
\text { 73FLAA-A } \\
\text { 73FLAA-A } \\
\text { 73FLA-A } \\
\text { 73FLA-A } \\
\text { 73FLA-A } \\
\text { 73FLA-A } \\
\text { 73FLA-A } \\
\text { 73FLA-A } \\
\text { 73FLA-A } \\
\text { 73FLA-A } \\
\text { 73FLA-A } \\
\text { 73FLA-A } \\
\text { 73FLA-A } \\
\text { 73FLAA-A } \\
\text { 73FLA-A } \\
\text { 73FLA-A } \\
\text { 73FLA-A } \\
\text { 73FLA-A } \\
\text { 73FLA-A } \\
\text { 73FLA-A } \\
\text { 73FLA-A } \\
\text { 73FLA-A } \\
\text { 73FLA-A } \\
\text { 73FLA-A } \\
\text { 73FLA-A } \\
\text { 73FLA-A } \\
\text { 73FLA-A } \\
\text { 73FLA-A } \\
\text { 73FLA-A } \\
\text { 73FLA-A } \\
\text { 73FLA-A } \\
\text { 73FLA-A } \\
\text { 73FLA-A } \\
\text { 73FLA-A } \\
\text { 73FLA-A } \\
\text { 73FLA-A } \\
\text { 73FLA-A } \\
\text { 73FLA-A } \\
\text { 73FLA-A } \\
\text { 73FLA-A } \\
\text { 73FLA-A } \\
\text { 73FLA-A }\end{array}$ \\
\hline
\end{tabular}


TABLE A-II (cont)

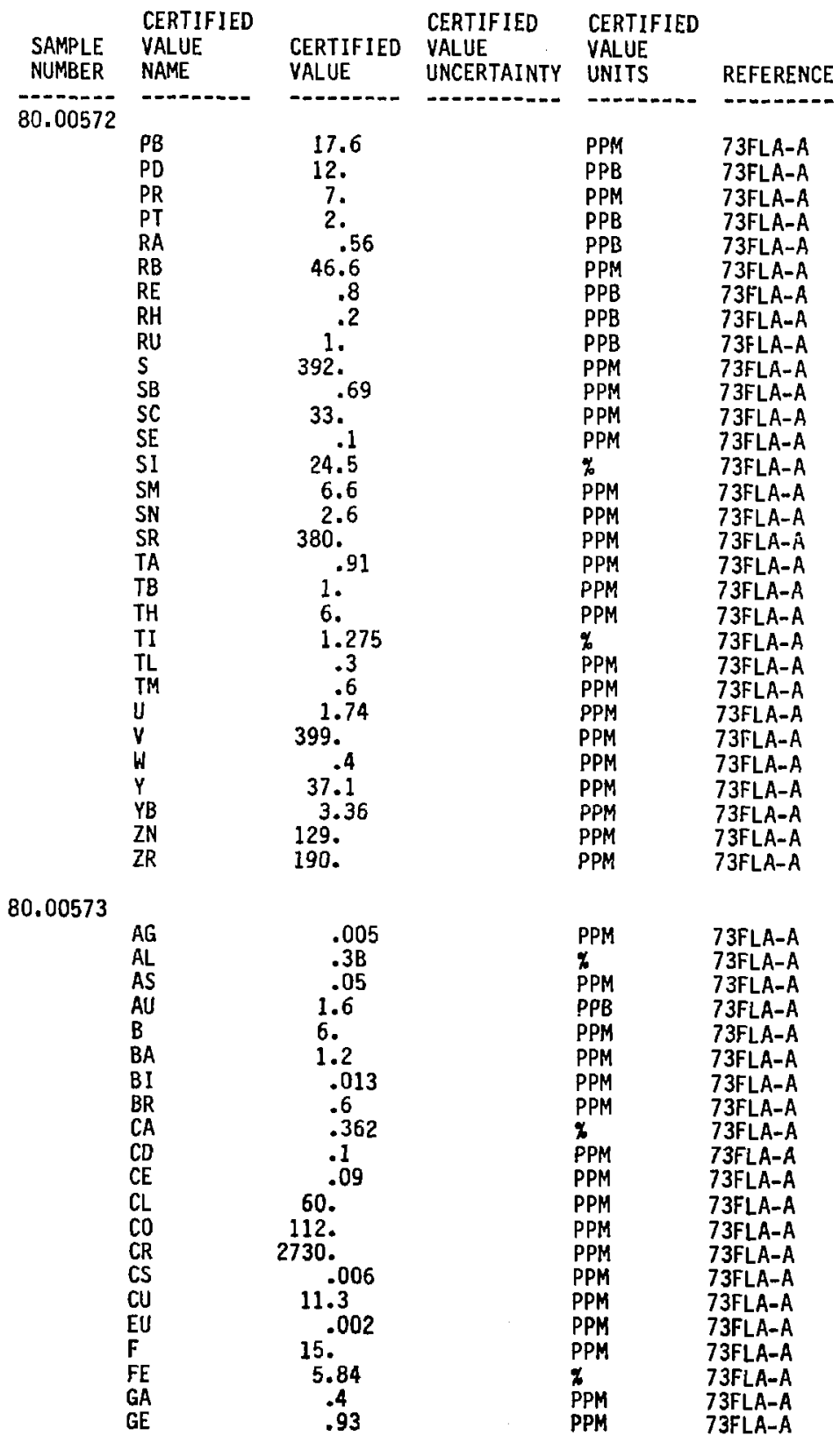


TABLE A-II (cont)

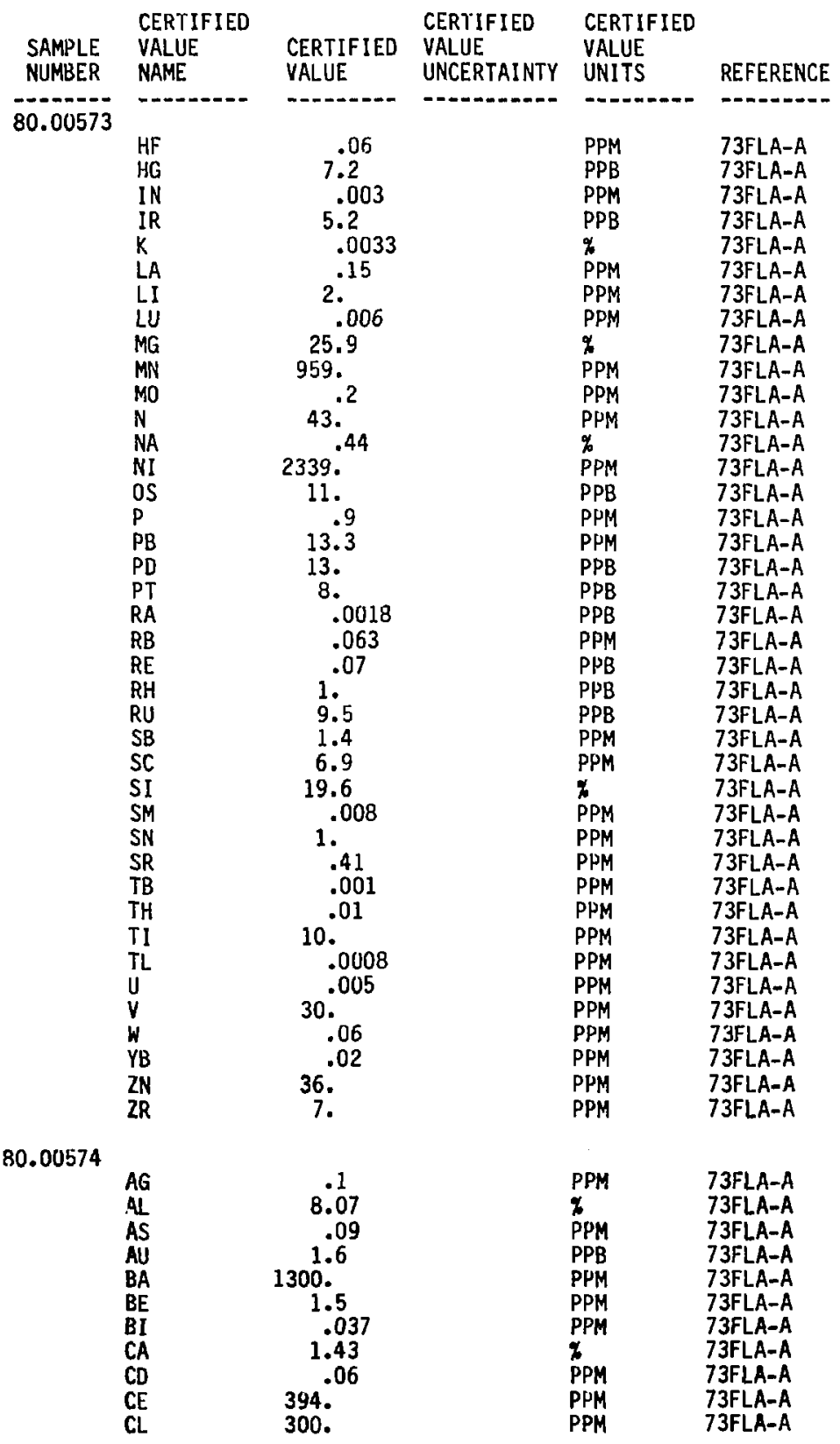


TABLE A-II (cont)

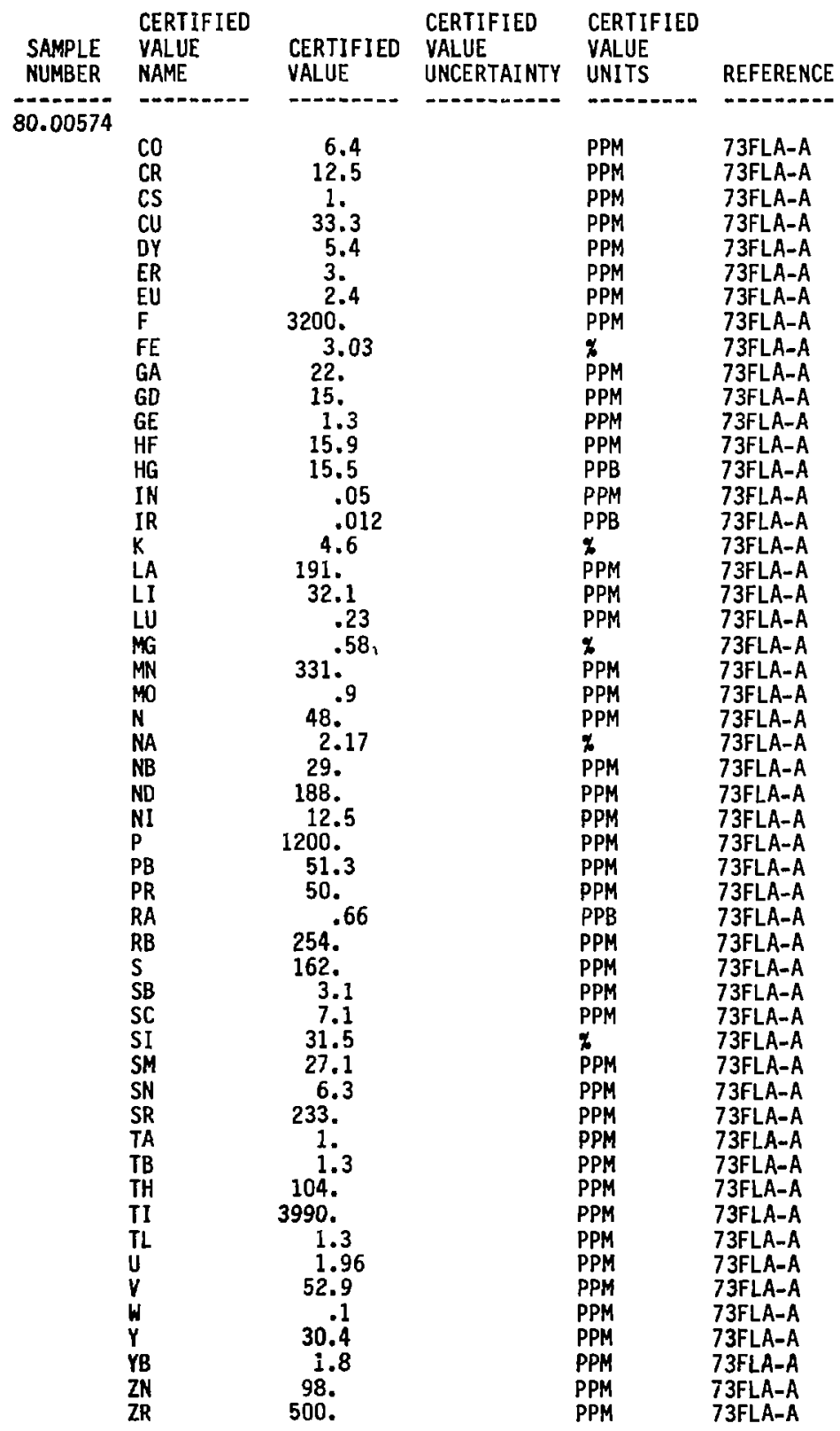


TABLE A-II (cont)

\begin{tabular}{|c|c|c|c|c|c|}
\hline $\begin{array}{l}\text { SAMPLE } \\
\text { NUMBER }\end{array}$ & $\begin{array}{l}\text { CERTIFIED } \\
\text { VALUE } \\
\text { NAME }\end{array}$ & $\begin{array}{l}\text { CERTIFIED } \\
\text { VALUE }\end{array}$ & $\begin{array}{l}\text { CERTIFIED } \\
\text { VALUE } \\
\text { UNCERTAINTY }\end{array}$ & $\begin{array}{l}\text { CERTIFIED } \\
\text { VALUE } \\
\text { UNITS }\end{array}$ & REFERENCE \\
\hline \multirow{2}{*}{$\begin{array}{l}80.00574 \\
80.00575\end{array}$} & & $\cdots$ & -.0 & $\cdots$ & - \\
\hline & $\begin{array}{l}A G \\
A L \\
A S \\
B \\
B A \\
B E \\
B R \\
C A \\
C D \\
C E \\
C D \\
C R \\
C S \\
C U \\
D Y \\
E U \\
F E \\
G A \\
H F \\
H G \\
K \\
L A \\
L I \\
M G \\
M D \\
N A \\
N I \\
P B \\
R B \\
S B \\
S C \\
S E \\
S I \\
S N \\
S R \\
T A \\
T E \\
T H \\
T I \\
U \\
V \\
Y B \\
Z N \\
Z R\end{array}$ & $\begin{array}{c}33 . \\
3.54 \\
460 . \\
15.3 \\
560 . \\
1.12 \\
.39 \\
870.3 \\
4 . \\
19 . \\
9.3 \\
10 . \\
4 . \\
1300 . \\
3.1 \\
.68 \\
24.7 \\
12 . \\
1.1 \\
3.9 \\
530 . \\
6.1 \\
7.7 \\
.21 \\
10 . \\
550 . \\
42 . \\
670 . \\
29 . \\
124 . \\
1.7 \\
18.6 \\
23 . \\
55 . \\
280 . \\
.2 \\
8.7 \\
2.3 \\
650 . \\
34.8 \\
88 . \\
1.8 \\
740 . \\
66 .\end{array}$ & $\begin{array}{c}2 . \\
.25 \\
30 . \\
.5 \\
120 . \\
.17 \\
.07 \\
800 . \\
.3 \\
4 . \\
1.1 \\
2 . \\
1.1 \\
100 . \\
.5 \\
.07 \\
1.8 \\
2 . \\
.3 \\
100 . \\
.3 \\
1.0 \\
.01 \\
2.0 \\
110 . \\
10 . \\
20 . \\
6 . \\
6 . \\
.1 \\
.8 \\
1.3 \\
1 . \\
60 . \\
.1 \\
.1 \\
.4 \\
50 . \\
.4 \\
9.4 \\
.5 \\
110 . \\
20 .\end{array}$ & $\begin{array}{l}P P M \\
\% \\
\text { PPM } \\
P P M \\
P P M \\
P P M \\
P P M \\
P P M \\
P P M \\
P P M \\
P P M \\
P P M \\
P P M \\
P P M \\
P P M \\
P P M \\
\% \\
P P M \\
P P M \\
P P M \\
P P M \\
P P M \\
P P M \\
\% \\
P P M \\
P P M \\
P P H \\
P P M \\
P P M \\
P P M \\
P P M \\
P P M \\
\chi \\
P P M \\
P P M \\
P P M \\
P P M \\
P P M \\
P P M \\
P P M \\
P P M \\
P P M \\
P P M \\
P P M\end{array}$ & $\begin{array}{l}\text { 80GLA-B } \\
\text { 79GLA-A } \\
\text { 79GLA-A } \\
\text { 79GLA-A } \\
\text { 79GLA-A } \\
\text { 79GLA-A } \\
\text { 79GLA-A } \\
\text { 79GLA-A } \\
\text { 80GLA-B } \\
\text { 79GLA-A } \\
\text { 79GLA-A } \\
\text { 79GLA-A } \\
\text { 79GLA-A } \\
\text { 79GLA-A } \\
\text { 79GLA-A } \\
\text { 79GLA-A } \\
\text { 79GLA-A } \\
\text { 79GLA-A } \\
\text { 79GLA-A } \\
\text { 79GLA-A } \\
\text { 79GLA-A } \\
\text { 79GLA-A } \\
\text { 80GLA-B } \\
\text { 79GLA-A } \\
\text { 79GLA-A } \\
\text { 79GLA-A } \\
\text { 79GLA-A } \\
\text { 79GLA-A } \\
\text { 79GLA-A } \\
\text { 79GLA-A } \\
\text { 79GLA-A } \\
\text { 79GLA-A } \\
\text { 79GLA-A } \\
\text { 80GLA-B } \\
\text { 79GLA-A } \\
\text { 79GLA-A } \\
\text { 80GLA-B } \\
\text { 79GLA-A } \\
\text { 79GLA-A } \\
\text { 79GLA-A } \\
\text { 79GLA-A } \\
\text { 79GLA-A } \\
\text { 79GLA-A } \\
\text { 79GLA-A }\end{array}$ \\
\hline 80.00577 & $\begin{array}{l}A G \\
A L \\
A S \\
B \\
B A \\
B E\end{array}$ & $\begin{array}{c}17.6 \\
18.6 \\
31 . \\
44 . \\
2000 . \\
1.64\end{array}$ & $\begin{array}{c}.6 \\
.4 \\
5 . \\
1 . \\
400 . \\
.09\end{array}$ & $\begin{array}{l}\text { PPM } \\
\% \\
\text { PPM } \\
\text { PPM } \\
\text { PPM } \\
\text { PPN }\end{array}$ & $\begin{array}{l}\text { 80GLA-B } \\
\text { 79GLA-A } \\
\text { 79GLA-A } \\
\text { 79GLA-A } \\
\text { 79GLA-A } \\
\text { 79GLA-A }\end{array}$ \\
\hline
\end{tabular}


TABLE A-II (cont)

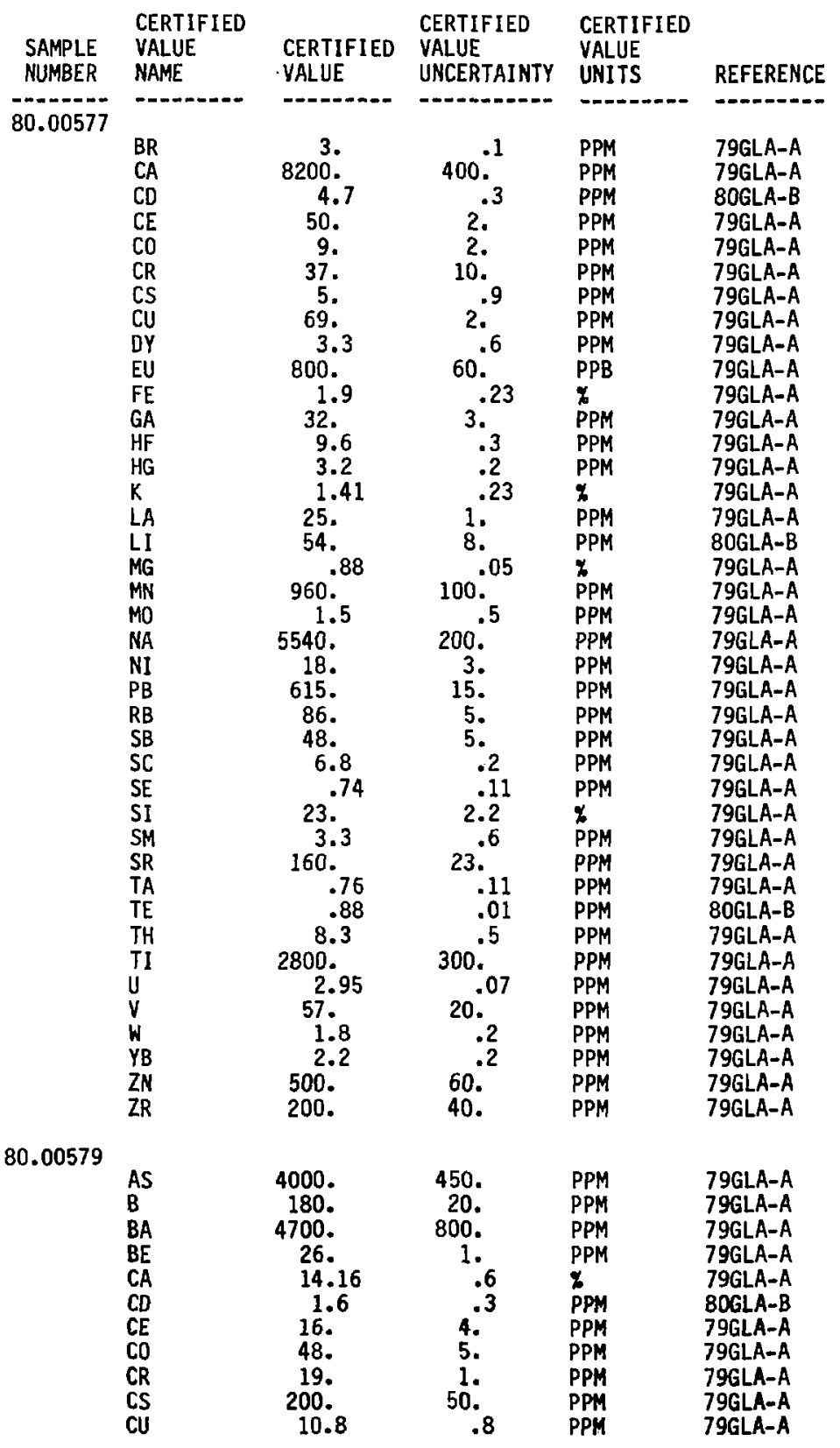


TABLE A-II (cont)

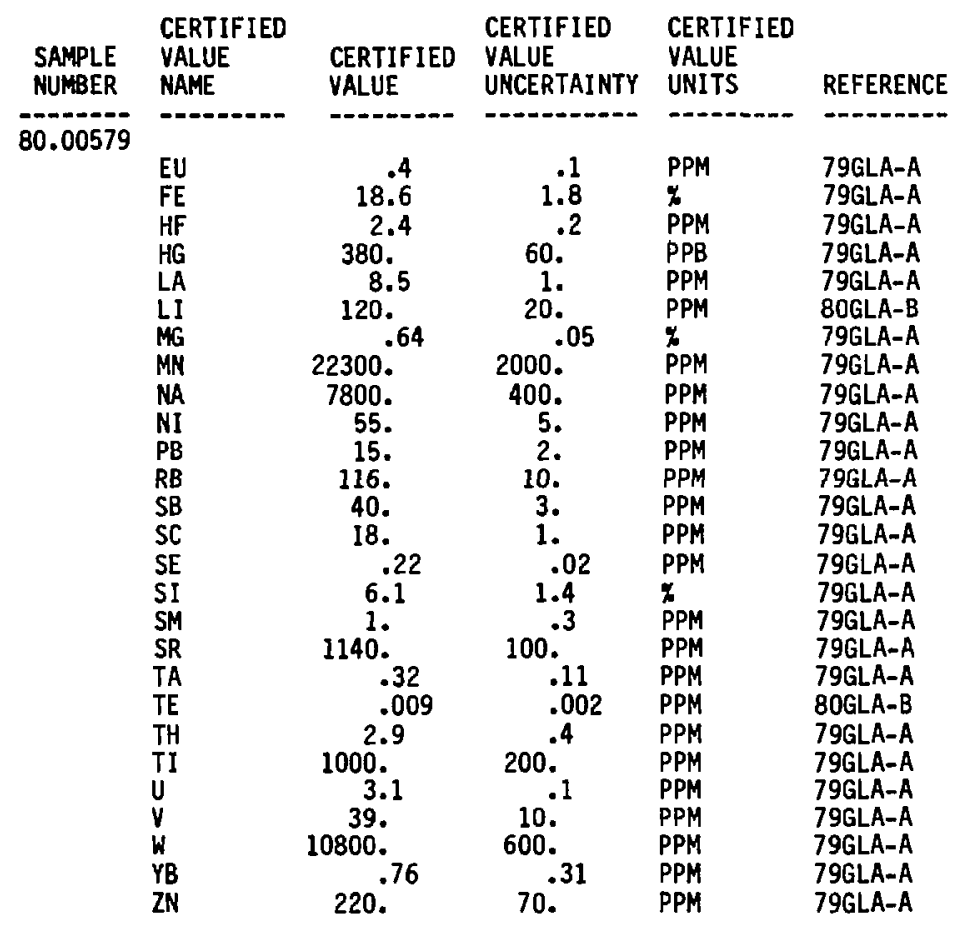

80.00581

\begin{tabular}{|c|c|c|c|c|}
\hline $\begin{array}{l}\text { AL } \\
A S \\
B \\
B A \\
B E \\
B R \\
C A \\
C D \\
C E \\
C O \\
C R \\
C S \\
C U \\
D Y \\
E U \\
F E \\
G A \\
H F \\
H G \\
K \\
L A \\
L I \\
\text { LI } \\
\text { MG } \\
\text { in }\end{array}$ & $\begin{array}{c}7.4 \\
98 . \\
4.3 \\
1350 . \\
2.1 \\
.5 \\
9000 . \\
500 . \\
114 . \\
16 . \\
64 . \\
3 . \\
6500 . \\
2.6 \\
1.6 \\
2.97 \\
15 . \\
8 . \\
.13 \\
4.3 \\
64 . \\
12 . \\
1.65 \\
140 .\end{array}$ & $\begin{array}{c}10.2 \\
.4 \\
330.4 \\
.2 \\
.11 \\
500 . \\
100 . \\
8 . \\
2 . \\
10 . \\
1 . \\
200 . \\
1.1 \\
.1 \\
.43 \\
1 . \\
.5 \\
.01 \\
.6 \\
5 . \\
3 . \\
.05 \\
20 .\end{array}$ & $\begin{array}{l}\% \\
P P M \\
P P M \\
P P M \\
P P M \\
P P M \\
P P M \\
P P B \\
P P M \\
P P M \\
P P M \\
P P M \\
P P M \\
P P M \\
P P M \\
x \\
P P M \\
P P M \\
P P M \\
x \\
P P M \\
P P M \\
x \\
P P M\end{array}$ & $\begin{array}{l}\text { 79GLA-A } \\
\text { 79GLA-A } \\
\text { 79GLA-A } \\
\text { 79GLA-A } \\
\text { 79GLA-A } \\
\text { 79GLA-A } \\
\text { 79GLA-A } \\
\text { 80GLA-B } \\
\text { 79GLA-A } \\
\text { 79GLA-A } \\
\text { 79GLA-A } \\
\text { 79GLA-A } \\
\text { 79GLA-A } \\
\text { 79GLA-A } \\
\text { 79GLA-A } \\
\text { 79GLA-A } \\
\text { 79GLA-A } \\
\text { 79GLAA-A } \\
\text { 796LA-A } \\
\text { 79GLA-A } \\
\text { 79GLA-A } \\
\text { 80GLA-B } \\
\text { 79GLA-A } \\
\text { 79GLA-A }\end{array}$ \\
\hline
\end{tabular}


TABLE A-II (cont)

\begin{tabular}{|c|c|c|c|c|c|}
\hline $\begin{array}{l}\text { SAMPLE } \\
\text { NUMBER }\end{array}$ & $\begin{array}{l}\text { CERTIFIEO } \\
\text { VALUE } \\
\text { MAME }\end{array}$ & $\begin{array}{l}\text { CERTIFIED } \\
\text { VALUE }\end{array}$ & $\begin{array}{l}\text { CERTIFIED } \\
\text { VALUE } \\
\text { UNCERTAINTY }\end{array}$ & $\begin{array}{l}\text { CERTIFIED } \\
\text { VALUE } \\
\text { UNITS. }\end{array}$ & REFERENCE \\
\hline .00581 & $\cdots$ & & $\ldots$ & -- & - \\
\hline sed & $\begin{array}{l}M O \\
N A \\
N I \\
R B \\
\text { RB } \\
S C \\
S E \\
S I \\
S M \\
S R \\
T A \\
T E \\
T H \\
T I \\
U \\
V \\
W \\
Y B \\
Z N \\
Z R\end{array}$ & $\begin{array}{c}310 . \\
5300 . \\
38 . \\
174 . \\
4.4 \\
8.3 \\
6 . \\
31.3 \\
6 . \\
220 . \\
. .77 \\
1 . . \\
22 . \\
2600 . \\
6.42 \\
92 . \\
28 . \\
1.8 \\
64 . \\
200 .\end{array}$ & $\begin{array}{c}25 . \\
300 . \\
4 . \\
7 . \\
.8 \\
.4 \\
.5 \\
2.1 \\
1 . \\
30 . \\
.07 \\
.1 \\
2 . \\
300 . \\
.25 \\
15 . \\
5 . \\
.1 \\
10 . \\
40 .\end{array}$ & $\begin{array}{l}\text { PPM } \\
\text { PPM } \\
\text { PPM } \\
\text { PPM } \\
\text { PPM } \\
\text { PPM } \\
\text { PPM } \\
\text { PPM } \\
\text { PPM } \\
\text { PPM } \\
\text { PPM } \\
\text { PPM } \\
\text { PPM } \\
\text { PPM } \\
\text { PPM } \\
\text { PPM } \\
\text { PPM } \\
\text { PPM } \\
\text { PPM }\end{array}$ & $\begin{array}{l}\text { 79GLA-A } \\
\text { 79GLA-A } \\
\text { 79GLA-A } \\
\text { 79GLA-A } \\
\text { 79GLA-A } \\
\text { 79GLA-A } \\
\text { 79GLA-A } \\
\text { 79GLA-A } \\
\text { 79GLA-A } \\
\text { 79GLA-A } \\
\text { 79GLA-A } \\
\text { 80GLA-B } \\
\text { 79GLA-A } \\
\text { 79GLA-A } \\
\text { 79GLA-A } \\
\text { 79GLA-A } \\
\text { 79GLA-A } \\
\text { 79GLA-A } \\
\text { 79GLA-A } \\
\text { 79GLA-A }\end{array}$ \\
\hline
\end{tabular}

80.00583

\begin{tabular}{|c|c|c|c|c|}
\hline $\begin{array}{l}A L \\
A S \\
B \\
B A \\
B E \\
B R \\
C A \\
C E \\
C 0 \\
C R \\
C S \\
C U \\
D Y \\
E U \\
F E \\
G A \\
H F \\
H G \\
L A \\
M G \\
M N \\
M D \\
N A \\
N I \\
P B \\
R B \\
S C \\
S E \\
S I \\
S M\end{array}$ & $\begin{array}{c}20.8 \\
12 . \\
25 . \\
1800 . \\
1.19 \\
7.8 \\
7500 . \\
40 . \\
30 . \\
100 . \\
2.2 \\
360 . \\
2 . \\
.94 \\
3.19 \\
34 . \\
6.2 \\
.17 \\
18 . \\
1.22 \\
280 . \\
30 . \\
7700 . \\
63 . \\
22 . \\
40 . \\
7.8 \\
1.1 \\
19.7 \\
2.9 \\
120 .\end{array}$ & $\begin{array}{c}1.6 \\
3 . \\
2 . \\
500 . \\
.16 \\
.9 \\
600 . \\
2 . \\
5 . \\
5 . \\
.8 \\
20 . \\
.4 \\
.06 \\
.29 \\
3 . \\
.4 \\
.02 \\
4.05 \\
20 . \\
4 . \\
300 . \\
7 . \\
2 . \\
4 . \\
.4 \\
.1 \\
1.2 \\
.7 \\
20 .\end{array}$ & $\begin{array}{l}\% \\
\text { PPM } \\
\text { PPM } \\
\text { PPM } \\
\text { PPM } \\
\text { PPM } \\
\text { PPM } \\
\text { PPM } \\
\text { PPM } \\
\text { PPM } \\
\text { PPM } \\
\text { PPM } \\
\text { PPM } \\
\text { PPM } \\
\chi \\
\text { PPM } \\
\text { PPM } \\
\text { PPM } \\
\text { PPM } \\
\% \\
\text { PPM } \\
\text { PPM } \\
\text { PPM } \\
\text { PPM } \\
\text { PPM } \\
\text { PPM } \\
\text { PPM } \\
\text { PPM } \\
\% \\
\text { PPM } \\
\text { PPM }\end{array}$ & $\begin{array}{l}\text { 79GLA-A } \\
\text { 79GLA-A } \\
\text { 79GLA-A } \\
\text { 79GLA-A } \\
\text { 79GLA-A } \\
\text { 79GLA-A } \\
\text { 79GLA-A } \\
\text { 79GLA-A } \\
\text { 79GLA-A } \\
\text { 79GLA-A } \\
\text { 79GLA-A } \\
\text { 79GLA-A } \\
\text { 79GLA-A } \\
\text { 79GLA-A } \\
\text { 79GLA-A } \\
\text { 79GLA-A } \\
\text { 79GLA-A } \\
\text { 79GLA-A } \\
\text { 79GLA-A } \\
\text { 79GLA-A } \\
\text { 79GLA-A } \\
\text { 79GLA-A } \\
\text { 79GLA-A } \\
\text { 79GLA-A } \\
\text { 79GLA-A } \\
\text { 79GLA-A } \\
\text { 79GLAA-A } \\
\text { 79GLA-A } \\
\text { 79GLA-A } \\
\text { 79GLA-A } \\
\text { 79GLA-A }\end{array}$ \\
\hline
\end{tabular}


TABLE A-II (cont)

\begin{tabular}{|c|c|c|c|c|c|}
\hline $\begin{array}{l}\text { SAMPLE } \\
\text { RUMBER }\end{array}$ & $\begin{array}{l}\text { CERTIFIED } \\
\text { VALUE } \\
\text { NAME }\end{array}$ & $\begin{array}{l}\text { CERTIFIED } \\
\text { VALUE }\end{array}$ & $\begin{array}{l}\text { CERTIFIED } \\
\text { VALUE } \\
\text { UNCERTAINTYY }\end{array}$ & $\begin{array}{l}\text { CERT IFIED } \\
\text { VALUE } \\
\text { UNITS }\end{array}$ & REFERENCE \\
\hline 80.00583 & --- & & -- & $\ldots$ & \\
\hline .00500 & $\begin{array}{l}T A \\
T H \\
T I \\
U \\
V \\
Y B \\
Y B \\
Z N \\
Z R \\
8200 .\end{array}$ & $\begin{array}{c}460 . \\
5.3 \\
2100 . \\
2.13 \\
60 . \\
2 . \\
50 . \\
140 . \\
.82\end{array}$ & $\begin{array}{c}80 . \\
.5 \\
300 . \\
.1 \\
20 . \\
.2 \\
5 . \\
20 . \\
1100 .\end{array}$ & $\begin{array}{l}\text { PPB } \\
\text { PPM } \\
\text { PPM } \\
\text { PPM } \\
\text { PPM } \\
\text { PPM } \\
\text { PPM } \\
\text { PPM } \\
\text { PPM }\end{array}$ & $\begin{array}{l}\text { 79GLA-A } \\
\text { 79GLA-A } \\
\text { 79GLA-A } \\
\text { 79GLA-A } \\
\text { 79GLA-A } \\
\text { 79GLA-A } \\
\text { 79GLA-A } \\
\text { 79GLA-A } \\
\text { 79GLA-A }\end{array}$ \\
\hline
\end{tabular}

80.00585

\begin{tabular}{|c|c|c|c|c|}
\hline $\begin{array}{l}A L \\
A S \\
B \\
B A \\
B E \\
B E \\
B R \\
C A \\
C E \\
C D \\
C R \\
C S \\
C U \\
D Y \\
E U \\
F E \\
G A \\
H F \\
H G \\
K \\
L A \\
M G \\
M N \\
M O \\
N A \\
N I \\
P B \\
R B \\
S B \\
S C \\
S E \\
S I \\
S A \\
S R \\
T A \\
T E \\
T H \\
T I \\
U \\
V \\
W \\
Y B \\
Z N\end{array}$ & $\begin{array}{c}16.6 \\
340 . \\
11 . \\
1100 . \\
1.1 \\
1.4 \\
970 . \\
38 . \\
14 . \\
96 . \\
4.8 \\
105 . \\
2.8 \\
780 . \\
5.58 \\
30 . \\
5.3 \\
80 . \\
2.04 \\
14 . \\
.62 \\
1000 . \\
1.7 \\
1040 . \\
22 . \\
110 . \\
107 . \\
3.8 \\
31 . \\
1.07 \\
22.9 \\
2.4 \\
42 . \\
520 . \\
.016 \\
5.2 \\
5000 . \\
1.58 \\
180 . \\
.88 \\
2.7 \\
120 .\end{array}$ & $\begin{array}{c}30.4 \\
1 . \\
300 . \\
.1 \\
100.3 \\
7 . \\
3 . \\
10 . \\
1.7 \\
12 . \\
.4 \\
400 . \\
.42 \\
6 . \\
.3 \\
10 . \\
.25 \\
2 . \\
.04 \\
50 . \\
.4 \\
60 . \\
4 . \\
10 . \\
7 . \\
.7 \\
1 . \\
.13 \\
1.7 \\
.6 \\
90 . \\
80 . \\
400.4 \\
20.08 \\
.18 . \\
.3 \\
20 .\end{array}$ & 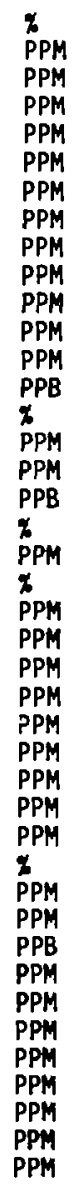 & 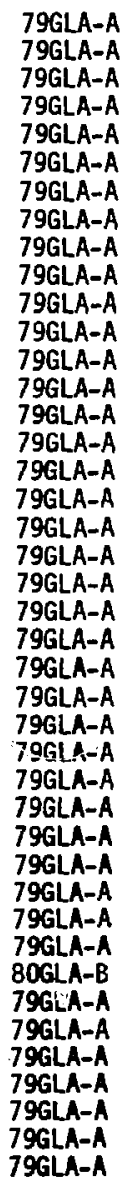 \\
\hline
\end{tabular}


TABLE A-II (cont)

\begin{tabular}{|c|c|c|c|c|c|}
\hline $\begin{array}{l}\text { SAMPLE } \\
\text { NUMBER }\end{array}$ & $\begin{array}{l}\text { CERTIFIED } \\
\text { VALUE } \\
\text { NAME }\end{array}$ & $\begin{array}{l}\text { CERTIFIED } \\
\text { VALUE }\end{array}$ & $\begin{array}{l}\text { CERTIFIED } \\
\text { VALUE } \\
\text { UNCERTAINTY }\end{array}$ & $\begin{array}{l}\text { CERT IF IED } \\
\text { VALUE } \\
\text { UNITS }\end{array}$ & REFERENCE \\
\hline \multirow[t]{2}{*}{80.00585} & , & $\cdots \cdots$ & - & $\cdots \cdots$ & $\cdots$ \\
\hline & ZR & 106. & 8. & PPM & 79GLA A \\
\hline \multicolumn{6}{|l|}{80.00587} \\
\hline & $\begin{array}{l}P B-21 \\
R A-226 \\
T H-23 \\
U\end{array}$ & $\begin{array}{l}23200 \\
24000 \\
22600 \\
7.09\end{array}$ & .03 & $\begin{array}{l}P C I / G \\
P C I / G \\
P C I / G \\
x\end{array}$ & $\begin{array}{l}\text { 79FAY-A } \\
\text { 79FAY-A } \\
\text { 79FAY-A } \\
\text { 79FAY -A }\end{array}$ \\
\hline \multicolumn{6}{|l|}{80.00588} \\
\hline & $R A-226$ & 983. & 18. & $\mathrm{PCI} / *$ & 68NBS-A \\
\hline 80.00589 & $\begin{array}{l}\mathrm{AL} \\
\mathrm{AS} \\
\mathrm{BE} \\
\mathrm{CD} \\
\mathrm{CO} \\
\mathrm{CR} \\
\mathrm{CU} \\
\mathrm{FE} \\
\mathrm{MN} \\
\mathrm{NI} \\
\mathrm{PB} \\
\mathrm{SE} \\
\mathrm{V} \\
\mathrm{ZN}\end{array}$ & $\begin{array}{l}79 . \\
26 . \\
16 . \\
5.2 \\
17 . \\
16 . \\
16 . \\
26 . \\
26 . \\
26 . \\
22 . \\
5.2 \\
52 . \\
11 .\end{array}$ & $\begin{array}{l}7.9 \\
2.6 \\
1.6 \\
.52 \\
1.7 \\
1.6 \\
1.6 \\
2.6 \\
2.6 \\
2.6 \\
2.2 \\
.52 \\
5.2 \\
1.1\end{array}$ & $\begin{array}{l}P P B \\
P P B \\
P P B \\
P P B \\
P P B \\
P P B \\
P P B \\
P P B \\
P P B \\
P P B \\
P P B \\
P P B \\
P P B \\
P P B\end{array}$ & $\begin{array}{l}\text { 75EPA-A } \\
\text { 75EPA-A } \\
\text { 75EPA-A } \\
\text { 75EPA-A } \\
\text { 75EPA-A } \\
\text { 75EPA-A } \\
\text { 75EPA-A } \\
\text { 75EPA-A } \\
\text { 75EPA-A } \\
\text { 75EPA-A } \\
\text { 75EPA-A } \\
\text { 75EPA-A } \\
\text { 75EPA-A } \\
\text { 75EPA-A }\end{array}$ \\
\hline 80.00590 & $\begin{array}{l}A L \\
A S \\
B E \\
C D \\
C D \\
C R \\
C U \\
F E \\
H G \\
M N \\
N I \\
P B \\
S E \\
V \\
Z N\end{array}$ & $\begin{array}{c}496 . \\
109 . \\
78 . \\
23 . \\
74 . \\
154 . \\
72 . \\
417 . \\
4.5 \\
45 . \\
45 . \\
298 . \\
26 . \\
104 . \\
30 .\end{array}$ & $\begin{array}{c}50 . \\
11 . \\
7.8 \\
2.3 \\
7.4 \\
15.4 \\
7.2 \\
42 . \\
.45 \\
4.5 \\
4.5 \\
30 . \\
2.6 \\
10 . \\
3 .\end{array}$ & $\begin{array}{l}\text { PPB } \\
\text { PPB } \\
\text { PPB } \\
\text { PPB } \\
\text { PPB } \\
\text { PPB } \\
\text { PPB } \\
\text { PPB } \\
\text { PPB } \\
\text { PPB } \\
\text { PPB } \\
\text { PPB } \\
\text { PPB } \\
\text { PPB } \\
\text { PPB }\end{array}$ & $\begin{array}{l}\text { 75EPA-A } \\
\text { 75EPA-A } \\
\text { 75EPA-A } \\
\text { 75EPA-A } \\
\text { 75EPA-A } \\
\text { 75EPA-A } \\
\text { 75EPA-A } \\
\text { 75EPA-A } \\
\text { 75EPA-A } \\
\text { 75EPA-A } \\
\text { 75EPA-A } \\
\text { 75EPA-A } \\
\text { 75EPA-A } \\
\text { 75EPA-A } \\
\text { 75EPA-A }\end{array}$ \\
\hline 80.00591 & $\begin{array}{l}\mathrm{AL} \\
\mathrm{AS} \\
\mathrm{BE} \\
\mathrm{CD} \\
\mathrm{CO} \\
\mathrm{CR} \\
\mathrm{CU} \\
\mathrm{FE}\end{array}$ & $\begin{array}{l}904 . \\
154 . \\
398 . \\
73 . \\
396 . \\
209 . \\
102 . \\
678 .\end{array}$ & $\begin{array}{l}90 . \\
15.4 \\
40 . \\
7.3 \\
40 . \\
21 . \\
10.2 \\
68 .\end{array}$ & $\begin{array}{l}\text { PPB } \\
P P B \\
P P B \\
P P B \\
P P B \\
P P B \\
P P B \\
P P B\end{array}$ & $\begin{array}{l}\text { 75EPA-A } \\
\text { 75EPA-A } \\
\text { 75EPA-A } \\
\text { 75EPA-A } \\
\text { 75EPA-A } \\
\text { 75EPA-A } \\
\text { 75EPA-A } \\
75 \mathrm{EPA}-\mathrm{A}\end{array}$ \\
\hline
\end{tabular}


TABLE A-II (cont)

\begin{tabular}{|c|c|c|c|c|c|}
\hline $\begin{array}{l}\text { SAMPLE } \\
\text { NUMBER }\end{array}$ & $\begin{array}{l}\text { CERTIFIED } \\
\text { VALUE } \\
\text { NAME }\end{array}$ & $\begin{array}{l}\text { CERTIFIED } \\
\text { VALUE }\end{array}$ & $\begin{array}{l}\text { CERTIFIED } \\
\text { VALUE } \\
\text { UNCERTAINTY }\end{array}$ & $\begin{array}{l}\text { CERTIFIED } \\
\text { VALUE } \\
\text { UNITS }\end{array}$ & REFERENCE \\
\hline \multirow{2}{*}{80.00591} & & $\cdots$ & - & $\cdots$ & \\
\hline & $\begin{array}{l}\mathrm{HG} \\
\text { MN } \\
\mathrm{NI} \\
\mathrm{PB} \\
\mathrm{SE} \\
V \\
\mathrm{ZN}\end{array}$ & $\begin{array}{l}9.4 \\
397 . \\
152 . \\
352 . \\
44 . \\
157 . \\
174 .\end{array}$ & $\begin{array}{l}.94 \\
40.9 \\
15.2 \\
35 . \\
4.4 \\
15.7 \\
17.4\end{array}$ & $\begin{array}{l}\text { PPB } \\
\text { PPB } \\
\text { PPB } \\
\text { PPB } \\
\text { PPB } \\
\text { PPB } \\
\text { PPB }\end{array}$ & $\begin{array}{l}\text { 75EPA-A } \\
\text { 75EPA-A } \\
\text { 75EPA-A } \\
\text { 75EPA-A } \\
\text { 75EPA-A } \\
\text { 75EPA-A } \\
\text { 75EPA-A }\end{array}$ \\
\hline
\end{tabular}

80.00592

$\begin{array}{lr}\text { CA } & 14.5 \\ \text { CL } & 28.1 \\ \text { F } & .2 \\ \text { HARD } & 48.6 \\ \text { K } & 2.7 \\ \text { MG } & 3 . \\ \text { NA } & 5 . \\ \text { PH } & 7.9 \\ \text { SO4 } & 12 . \\ \text { SP.C } & 157 . \\ \text { TDS } & 71.7 \\ \text { T.A. } & 10.4\end{array}$

$\begin{array}{lll}1.5 & \text { PPM } & \text { 74EPA-A } \\ 2.8 & \text { PPM } & \text { 74EPA-A } \\ .02 & \text { PPM } & \text { 74EPA-A } \\ 4.9 & \text { PPM * } & \text { 74EPA-A } \\ .3 & \text { PPM } & \text { 74EPA-A } \\ .3 & \text { PPM } & \text { 74EPA-A } \\ .5 & \text { PPM } & \text { 74EPA-A } \\ .1 & \text { PH } & \text { 74EPA-A } \\ 1.2 & \text { PPM } & \text { 74EPA-A } \\ 15.7 & \text { UMOHS } & \text { 74EPA-A } \\ 7.2 & \text { PPM } & \text { 74EPA-A } \\ 1.2 & \text { PPM * } & \text { 74EPA-A }\end{array}$

80.00593

CA $\quad 44.5$

$\begin{array}{ll}C L & 86.4\end{array}$

$\begin{array}{lr}F & 1.1 \\ \text { HARD } & 170.2\end{array}$

K 8.4

MG $\quad 14.4$

NA $\quad 39.7$

$\mathrm{PH} \quad 8.4$

$504 \quad 102.4$

SP.C. $\quad 603$.

TDS $\quad 318.3$

T.A. $\quad 35.7$

$\begin{array}{clc}4.5 & \text { PPM } & \text { 74EPA-A } \\ 8.6 & \text { PPM } & \text { 74EPA-A } \\ 1.1 & \text { PPM } & \text { 74EPA-A } \\ 17 . & \text { PPM * } & \text { 74EPA-A } \\ .8 & \text { PPM } & \text { 74EPA-A } \\ 1.4 & \text { PPM } & \text { 74EPA-A } \\ 4 . & \text { PPM } & 74 E P A-A \\ .1 & \text { PH } & 74 E P A-A \\ 10.2 & \text { PPM } & \text { 74EPA-A } \\ 60 . & \text { UMOHS } & 74 E P A-A \\ 31.8 & \text { PPM } & 74 E P A-A \\ 3.6 & \text { PPM * } & 74 E P A-A\end{array}$

80.00594

$\begin{array}{lc}\text { AL } & 9.38 \\ C A & 1.8 \\ C R & 160 . \\ C U & 61 . \\ \text { FE } & 6 . \\ \text { HG } & 22 . \\ K & 2.68 \\ \text { NG } & 2.31 \\ \text { MN } & 890 . \\ \text { NI } & 94 . \\ \text { P } & 620 . \\ \text { PB } & 21 . \\ \text { SI } & 25.7 \\ \text { TI } & 5300 . \\ U & 1.6 \\ V & 139 .\end{array}$

$\begin{array}{cll}.18 & \% & 79 B O W-A \\ .08 & \% & 79 B O W-A \\ 9 . & \text { PPM } & 79 B O W-A \\ 2 . & \text { PPM } & 79 B O W-A \\ .16 & \% & 79 B O W-A \\ 2.0 & \text { PPB } & 79 B O W-A \\ .06 & \% & 79 B O W-A \\ .07 & \% & 79 B O W-A \\ 33 . & \text { PPM } & 79 B O W-A \\ 5 . & \text { PPM } & 79 B O W-A \\ 56 . & \text { PPM } & 79 B O W-A \\ 3 . & \text { PPM } & 79 B O W-A \\ .2 & \% & 79 B O W-A \\ 220 . & \text { PPM } & 79 B O W-A \\ 12.1 & \text { PPM } & 79 B O W-A \\ 12 . & \text { PPM } & 79 B O W-A\end{array}$


TABLE A-II (cont)

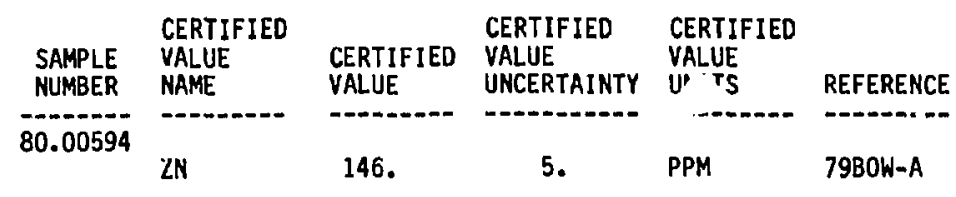

80.00596

$\begin{array}{lc}\text { AL } & 8.07 \\ \text { CA } & 1.96 \\ \text { CR } & 16 . \\ \text { CU } & 7 . \\ \text { FE } & 5.56 \\ \text { HG } & 82 . \\ \text { K } & 2.45\end{array}$

MG 5400 .

MN 720.

PB 21.

SI 25.

SR 340 .

TI 8600 .

v

ZN

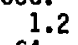

64.
124.

$\begin{array}{cl}.24 & x \\ .08 & x \\ 2 . & \text { PPM } \\ 1 . & \text { PPM } \\ 6.14 & \% \\ 6.06 & \text { PPB } \\ 300 . & \% \\ 22 . & \text { PPM } \\ 3 . & \text { PPM } \\ .0 & \text { PPM } \\ 25 . & \text { PPM } \\ 340 . & \text { PPM } \\ 7 . & \text { PPM } \\ 3 . & \text { PPM } \\ 3 . & \text { PPM }\end{array}$

79BOW-A

79BOW-A

79BOW-A

79BOW-A

79BOW-A

79BOW-A

79BOW-A

79BOW-A

79BOW-A

79BOW-A

79BOW-A

79BOW-A

79BOW-A

79BOW-A

79BOW-A

79BOW-A

80.00598

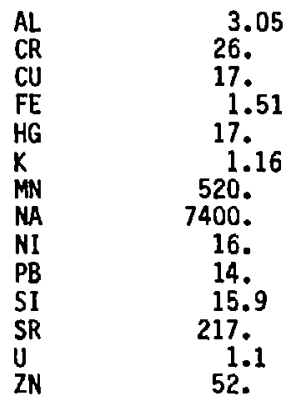

$\begin{array}{cl}.15 & \% \\ 3 . & \text { PPM } \\ 1.05 & \text { PPM } \\ 4.06 & \% \\ 4.03 & \text { PPB } \\ 17.03 & \% \\ 200 . & \text { PPM } \\ 2 . & \text { PPM } \\ 2 . & \text { PPM } \\ 17.2 & \% \\ 17 . & \text { PPM } \\ 3 . & \text { PPM } \\ & \text { PPM }\end{array}$

79BOW-A

79BOW-A

79BOW-A

79BOW-A

79BOW-A

7980W-A

79BOW-A

79BOW-A

79BOW-A

79BOW-A

79BOW-A

79BOW-A

79BOW-A

79BOW-A

80.00600

\begin{tabular}{|c|c|c|c|c|}
\hline $\begin{array}{l}\mathrm{AL} \\
\mathrm{CA} \\
\mathrm{CR} \\
\mathrm{CU} \\
\mathrm{FE} \\
\mathrm{K} \\
\mathrm{MG} \\
\mathrm{MN} \\
\mathrm{NI} \\
\mathrm{P} \\
\mathrm{PB} \\
\mathrm{SR} \\
\mathrm{TI}\end{array}$ & $\begin{array}{c}5.46 \\
1.11 \\
61 . \\
22 . \\
2.37 \\
1.73 \\
5600 . \\
600 . \\
26 . \\
900 . \\
16 . \\
170 . \\
3400 . \\
2.3 \\
90 . \\
94 .\end{array}$ & $\begin{array}{c}.1 \\
.03 \\
6 . \\
2 . \\
.05 \\
.04 \\
300 . \\
27 . \\
2 . \\
70 . \\
2 . \\
10 . \\
160 . \\
.3 \\
6 . \\
3 .\end{array}$ & $\begin{array}{l}\% \\
\varnothing \\
\text { PPM } \\
\text { PPM } \\
x \\
\varnothing \\
\text { PPM } \\
\text { PPM } \\
\text { PPM } \\
\text { PPM } \\
\text { PPM } \\
\text { PPM } \\
\text { PPM } \\
\text { PPM } \\
\text { PPM } \\
\text { PPM }\end{array}$ & 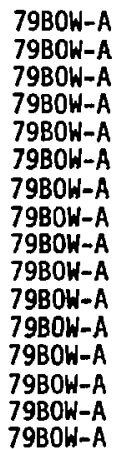 \\
\hline
\end{tabular}


TABLE A-II (cont)

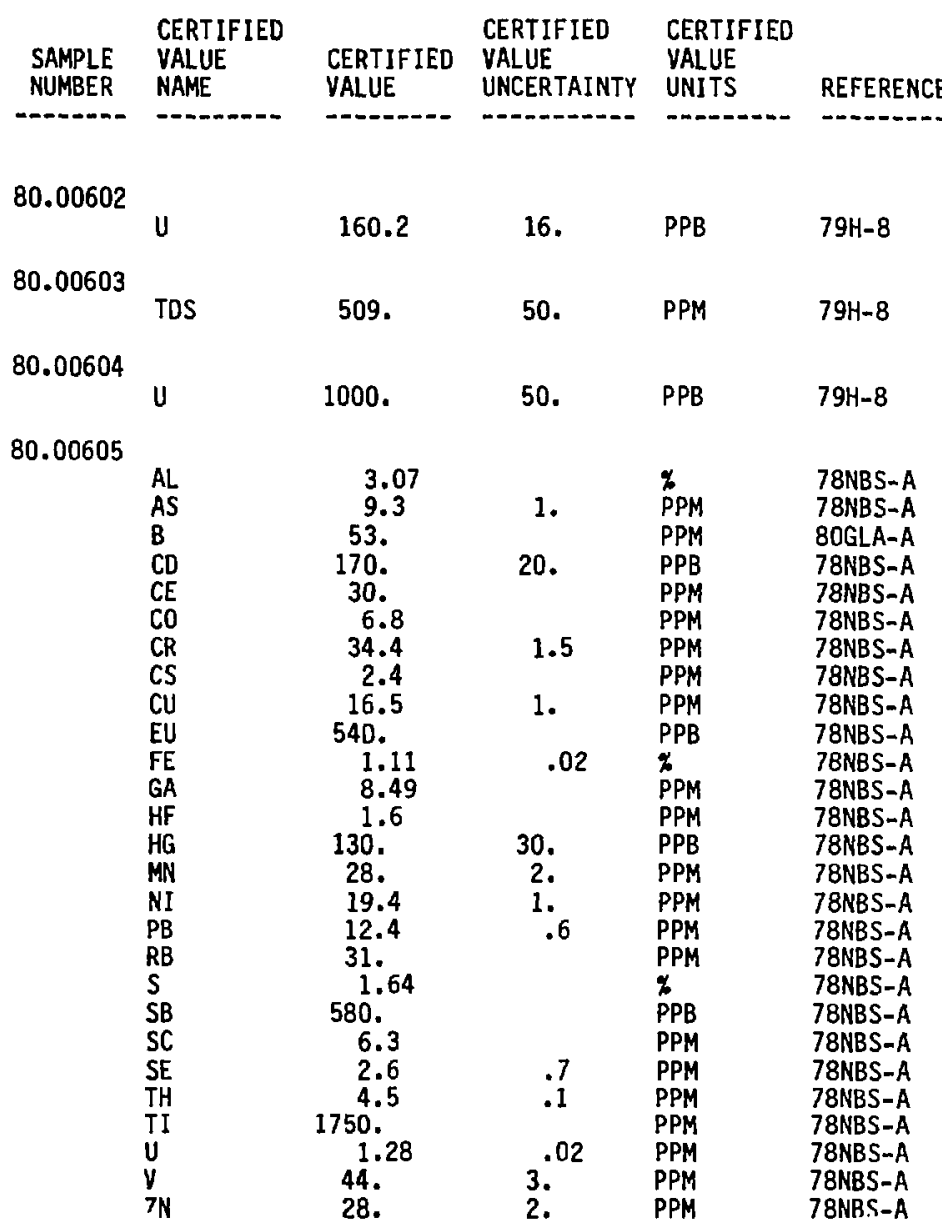

80.00606

\begin{tabular}{|c|c|c|c|c|}
\hline $\begin{array}{l}\text { AL } \\
\text { AS } \\
\text { CD } \\
\text { CE } \\
\text { CO } \\
\text { CR } \\
C U \\
\text { EU } \\
\text { FE } \\
\text { GA } \\
\text { HF } \\
\text { MN } \\
\text { NI }\end{array}$ & $\begin{array}{c}3200 . \\
420 . \\
30 . \\
3.6 \\
650 . \\
2.5 \\
3.6 \\
64 . \\
2390 . \\
1.05 \\
290 . \\
21.4 \\
1.74\end{array}$ & $\begin{array}{r}.3 \\
.3 \\
50 .\end{array}$ & $\begin{array}{l}\text { PPM } \\
\text { PPB } \\
\text { PPB } \\
\text { PPM } \\
\text { PPB } \\
\text { PPM } \\
\text { PPM } \\
\text { PPB } \\
\text { PPM } \\
\text { PPM } \\
\text { PPB } \\
\text { PPM } \\
\text { PPM }\end{array}$ & $\begin{array}{l}\text { 78NBS-B } \\
\text { 78NBS-B } \\
78 \mathrm{NBS}-\mathrm{B} \\
\text { 78NBS-B } \\
\text { 78NBS-B } \\
\text { 78NBS-B } \\
\text { 78NBS-B } \\
\text { 78NBS-B } \\
\text { 78NBS-B } \\
\text { 78NBS-B } \\
\text { 78NBS-B } \\
\text { 78NBS-B } \\
\text { 78NBS-B }\end{array}$ \\
\hline
\end{tabular}


TABLE A-II (cont)

\begin{tabular}{clccll} 
SAMPLE & $\begin{array}{l}\text { CERTIFIED } \\
\text { VALUE }\end{array}$ & $\begin{array}{l}\text { CERTIFIED } \\
\text { CERTIFIED } \\
\text { VALUE }\end{array}$ & $\begin{array}{l}\text { CERTIFIED } \\
\text { VALUE } \\
\text { UNCERTAINTY }\end{array}$ & $\begin{array}{l}\text { VALUE } \\
\text { UNITS }\end{array}$ & REFERENCE \\
\hline 80.00606 & & & & & \\
& PB & 1.9 & .2 & PPM & 78NBS-B \\
& S & 3300. & & PPM & 78NBS-B \\
& SB & 140. & & PPB & 78NBS-B \\
& SC & 630. & & PPB & 78NBS-B \\
& SE & 900. & 300. & PPB & 78NBS-B \\
& TH & 620. & 40. & PPB & 78NBS-B \\
& TI & 200. & & PPM & 78NBS-B \\
& U & 240. & 20. & PPB & 78NBS-B \\
& V & 5.2 & .5 & PPM & 78NBS-B \\
& ZN & 4.7 & .5 & PPM & 78NBS-B
\end{tabular}

80.00607

$\begin{array}{lr}\text { AG } & 6 . \\ \text { AL } & 3.3 \\ \text { AS } & 115 . \\ \text { BA } & 737 . \\ \text { BR } & 500 . \\ \text { CD } & 75 . \\ \text { CE } & 55 . \\ \text { CL } & 4500 . \\ \text { CO } & 18 . \\ \text { CR } & 403 . \\ \text { CS } & 3 . \\ \text { CU } & 609 . \\ \text { EU } & .8\end{array}$

$\mathrm{FE} \quad 3.91$

$\mathrm{HF}$

I

IN

LA

4.4

20.

1.

1.

42.

8000 .

860 .

4000 .

82.

6550.
45.

7.

24.

12.5

4.4
7.4

4000.

5.5

130.

4.8

3

0.

5.

.

3.

.

8

4

$.5 \quad P P M$

BNBS-B

4760 .

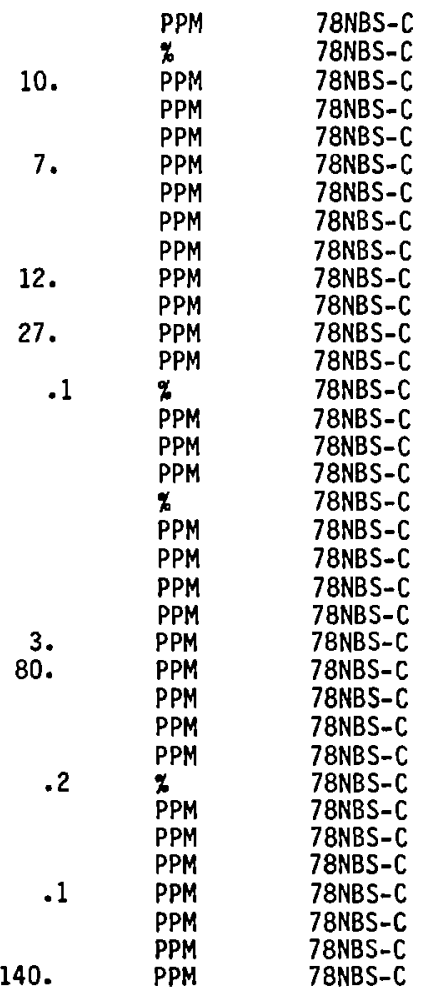

80.00608

$\begin{array}{lr}\text { AL } & 2.1 \\ \text { AS } & 66 . \\ \text { CA } & 2.9 \\ \text { CD } & 10.2\end{array}$

$\begin{array}{lll} & & \\ & \text { PPM } & \text { 78NBS-D } \\ & \% & \text { 78NBS-D } \\ 1.5 & \text { PPM } & \text { 78NBS-D } \\ 1.5 & \text { PPNBS-D }\end{array}$


TABLE A-II (cont)

\begin{tabular}{|c|c|c|c|c|c|}
\hline \multirow{2}{*}{$\begin{array}{c}\text { SAMPLE } \\
\text { NUMBER } \\
80.00608\end{array}$} & $\begin{array}{l}\text { CERTIFIED } \\
\text { VALUE } \\
\text { NAME }\end{array}$ & $\begin{array}{l}\text { CERTIFIED } \\
\text { VALUE }\end{array}$ & $\begin{array}{l}\text { CERTIFIED } \\
\text { VALUE } \\
\text { UNCERTAINTY }\end{array}$ & $\begin{array}{l}\text { CERTIFIED } \\
\text { VALUE } \\
\text { UNITS }\end{array}$ & REFERENCE \\
\hline & & & & & \\
\hline & $\mathrm{CO}$ & 8. & & PPM & 78NBS-D \\
\hline & $C R$ & 2.96 & .28 & & 78NBS-D \\
\hline & CU & 109. & 19. & PPM & 78NBS-D \\
\hline & $\mathrm{FE}$ & 11.3 & 1.2 & $\%$ & $78 N B S-D$ \\
\hline & HG & 1.1 & .5 & PPM & 78NBS-D \\
\hline & $k$ & 1.2 & & & 78NBS-D \\
\hline & LI & 9. & & PPM & 78NBS-0 \\
\hline & $M G$ & 2.4 & & & 78NBS-D \\
\hline & MN & 785. & 97. & PPM & 78NBS-D \\
\hline & $N$ & 797. & 48. & PPM & 78NBS-D \\
\hline & NA & 5500. & & PPM & $\begin{array}{l}\text { 78NBS-D } \\
78 N B S-0\end{array}$ \\
\hline & ${ }_{p}^{N 1}$ & $\begin{array}{l}43.8 \\
510 .\end{array}$ & 14.9 & PPM & 78NBS-D \\
\hline & PB & 714. & 28. & PPM & $78 N B S-L$ \\
\hline & $S B$ & 51. & & PPM & 78NBS-D \\
\hline & SC & 2. & & PPM & 78NBS-D \\
\hline & SI & 24. & & & 78NBS-D \\
\hline & TH & 1.62 & .22 & PPM & 78NBS-D \\
\hline & $u$ & 1.44 & .07 & $\begin{array}{l}\text { PPM } \\
\text { PPM }\end{array}$ & $\begin{array}{l}\text { 78NBS-D } \\
78 N B S=D\end{array}$ \\
\hline & v & 23.5 & 6.95 & $\begin{array}{l}\text { PPM } \\
\text { PPM }\end{array}$ & $\begin{array}{l}18 N B S-D \\
78 N B S-D\end{array}$ \\
\hline & ZN & 1720 & $169^{\circ}$ & PPM & 78NBS-D \\
\hline
\end{tabular}

80.00609

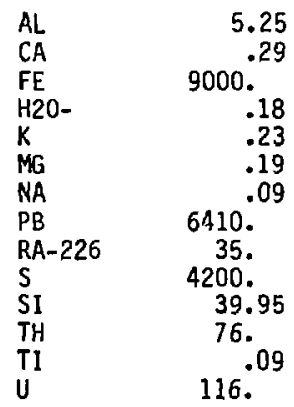

$\begin{array}{ll}\% & \text { 80STE-A } \\ \% & \text { 80STE-A } \\ \text { PPM } & \text { 80STE-A } \\ \% & \text { 80STE-A } \\ \% & \text { 80STE-A } \\ \% & 80 \text { STE-A } \\ \% & 80 \text { STE-A } \\ \text { PPM } & \text { 80STE-A } \\ \text { PCI /G } & \text { 80STE-A } \\ \text { PPM } & \text { 80STE-A } \\ \text { PPM } & \text { 8OSTE-A } \\ \text { PPM } & \text { 80STE-A } \\ \% & \text { 80STE-A } \\ \text { PPM } & \text { 80STE-A }\end{array}$

80.00610

\begin{tabular}{|c|c|c|c|c|}
\hline $\begin{array}{l}A L \\
A U \\
B A \\
B I \\
C \\
C A \\
C E \\
C L \\
C O \\
C R \\
C U \\
D Y \\
E R\end{array}$ & $\begin{array}{c}7.3 \\
1.5 \\
142 . \\
15 . \\
120 . \\
8.18 \\
41 . \\
92 . \\
45 . \\
300 . \\
137 . \\
4.8\end{array}$ & $\begin{array}{c}.12 \\
.3 \\
18 . \\
4 . \\
60 . \\
.12 \\
4 . \\
4 . \\
1 . \\
30 . \\
6 . \\
.2 \\
.3\end{array}$ & $\begin{array}{l}\% \\
\text { PPB } \\
\text { PPM } \\
\text { PPB } \\
\text { PPM } \\
\% \\
\text { PPM } \\
\text { PPM } \\
\text { PPM } \\
\text { PPM } \\
\text { PPM } \\
\text { PPM } \\
\text { PPM }\end{array}$ & $\begin{array}{l}\text { 81GLA-A } \\
\text { 81GLA-A } \\
\text { 81GLA-A } \\
\text { 81GLA-A } \\
\text { 81GLA-A } \\
\text { 81GLA-A } \\
\text { 81GLA-A } \\
\text { 81GLA-A } \\
\text { 81GLA-A } \\
\text { 81GLA-A } \\
\text { 81GLA-A } \\
\text { 81GLA-A } \\
\text { 81GLA-A }\end{array}$ \\
\hline
\end{tabular}


TABLE A-II (cont)

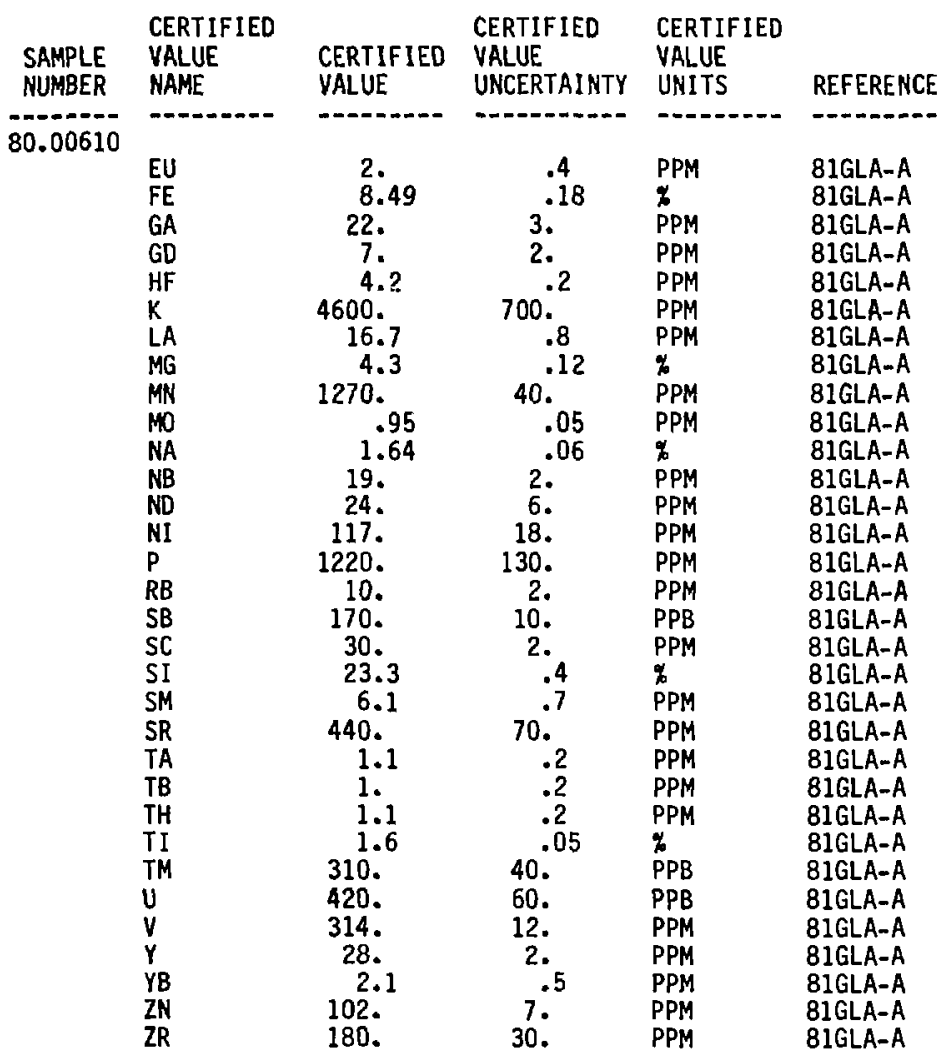

80.00611

$\begin{array}{lccll}\text { AL } & 8.65 & .15 & \% & \text { 81GLA-A } \\ \text { B } & 138 . & 15 . & \text { PPM } & \text { 81GLA-A } \\ \text { BA } & 490 . & 35 . & \text { PPM } & \text { 81GLA-A } \\ \text { BE } & 2.84 & .13 & \text { PPM } & \text { 81GLA-A } \\ \text { C } & 2.34 & .1 & \% & \text { 81GLA-A } \\ \text { CA } & 1.04 & .2 & \% & 81 G L A-A \\ \text { CE } & 94 . & 7 . & \text { PPM } & 81 G L A-A \\ \text { CL } & 3.12 & .04 & \% & 81 G L A-A \\ \text { CO } & 20 . & 2 . & \text { PPM } & \text { 81GLA-A } \\ \text { CR } & 105 . & 13 . & \text { PPM } & \text { 81GLA-A } \\ \text { CS } & 8.3 & .5 & \text { PPM } & \text { 81GLA-A } \\ \text { CU } & 30 . & 3 . & \text { PPM } & \text { 81GLA-A } \\ \text { EU } & 1.6 & .2 & \text { PPM } & \text { 81GLA-A } \\ \text { FE } & 4.86 & .26 & \% & \text { 81GLA-A } \\ \text { GA } & 21.4 & .4 & \text { PPM } & \text { 81GLA-A } \\ \text { GD } & 7.4 & 2 . & \text { PPM } & \text { 81GLA-A } \\ \text { HF } & 3.6 & .7 & \text { PPM } & \text { 81GLA-A } \\ \text { K } & 3 . & .2 & \% & \text { 81GLA-A } \\ \text { LI } & 76 . & 4 . & \text { PPM } & \text { 81GLA-A }\end{array}$


TABLE A-II (cont)

\begin{tabular}{|c|c|c|c|c|c|}
\hline $\begin{array}{l}\text { SAMPLE } \\
\text { NUMBER }\end{array}$ & $\begin{array}{l}\text { CERTIFIEO } \\
\text { VALUE } \\
\text { NAME }\end{array}$ & $\begin{array}{l}\text { CERTIFIED } \\
\text { VALUE }\end{array}$ & $\begin{array}{l}\text { CERTIF IED } \\
\text { YALUE } \\
\text { UNCERTAINTY }\end{array}$ & $\begin{array}{l}\text { CERTIFIED } \\
\text { VALUE } \\
\text { UNITS }\end{array}$ & REFERENCE \\
\hline 00611 & $\ldots-$ & $\ldots$ & $\ldots$ & $\ldots$ & 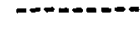 \\
\hline & $\begin{array}{l}M G \\
M N \\
N A \\
N B \\
N D \\
N I \\
P \\
P B \\
R B \\
\text { S } \\
\text { SB } \\
\text { SC } \\
\text { SE } \\
\text { SI } \\
\text { SM } \\
\text { SR } \\
\text { TA } \\
\text { TB } \\
\text { TH } \\
\text { TI } \\
U \\
V \\
Y B \\
Z N \\
Z R\end{array}$ & $\begin{array}{c}1.86 \\
810 . \\
2.77 \\
9.5 \\
44 . \\
54 . \\
760 . \\
25 . \\
152 . \\
4600 . \\
.91 \\
17.91 \\
1.15 \\
23.4 \\
7.8 \\
156 . \\
1 . \\
1.02 \\
12.8 \\
4300 . \\
2.85 \\
142 . \\
3 . \\
126 . \\
130 .\end{array}$ & $\begin{array}{c}160.12 \\
.19 \\
1.4 \\
3 . \\
8 . \\
65 . \\
4 . \\
3 . \\
600 . \\
.09 \\
2 . \\
.16 \\
.5 \\
.9 \\
19 . \\
.2 \\
.05 \\
.5 \\
200 . \\
.02 \\
3 . \\
.3 \\
18 . \\
10 .\end{array}$ & $\begin{array}{l}\% \\
\text { PPM } \\
x \\
\text { PPM } \\
\text { PPM } \\
\text { PPM } \\
\text { PPM } \\
\text { PPM } \\
\text { PPM } \\
\text { PPM } \\
\text { PPM } \\
\text { PPM } \\
\text { PPM } \\
x \\
\text { PPM } \\
\text { PPM } \\
\text { PPM } \\
\text { PPM } \\
\text { PPM } \\
\text { PPM } \\
\text { PPM } \\
\text { PPM } \\
\text { PPM } \\
\text { PPM } \\
\text { PPM }\end{array}$ & $\begin{array}{l}\text { 81GLA-A } \\
\text { 81GLA-A } \\
\text { 81GLA-A } \\
\text { 81GLA-A } \\
\text { 81GLA-A } \\
\text { 81GLA-A } \\
\text { 81GLA-A } \\
\text { 81GLA-A } \\
\text { 81GLA-A } \\
\text { 81GLA-A } \\
\text { 81GLA-A } \\
\text { 81GLA-A } \\
\text { 81GLA-A } \\
\text { 81GLA-A } \\
\text { 81GLA-A } \\
\text { 81GLA-A } \\
\text { 81GLA-A } \\
\text { 81GLA-A } \\
\text { 81GLA-A } \\
\text { 81GLA-A } \\
\text { 81GLA-A } \\
\text { 81GLA-A } \\
\text { 81GLA-A } \\
\text { 81GLA-A } \\
\text { 81GLA-A }\end{array}$ \\
\hline
\end{tabular}

80.00612

\begin{tabular}{|c|c|c|c|c|}
\hline $\begin{array}{l}A L \\
B \\
B A \\
B E \\
C \\
C A \\
C E \\
C L \\
C 0 \\
C S \\
C U \\
E U \\
F \\
F E \\
G A \\
G D \\
H F \\
K \\
M G \\
M N \\
M D \\
M A \\
N A \\
N B \\
N D \\
P \\
P B\end{array}$ & $\begin{array}{c}8.6 \\
38 . \\
1400 . \\
1.9 \\
29 . \\
2.27 \\
57 . \\
214 . \\
7.2 \\
1.8 \\
29 . \\
1.55 \\
275 . \\
3.02 \\
18 . \\
5.2 \\
4.4 \\
2.98 \\
6100 . \\
720 . \\
2.6 \\
3.09 \\
11 . \\
27 . \\
1100 . \\
21\end{array}$ & $\begin{array}{c}.08 \\
4 . \\
20 . \\
.2 \\
2 . \\
.06 \\
3 . \\
14 . \\
.4 \\
.2 \\
3 . \\
.12 \\
22.09 \\
.09 \\
1.4 \\
.2 \\
.09 \\
400 . \\
70 . \\
.4 \\
.1 \\
2 . \\
7 . \\
80 . \\
1 .\end{array}$ & $\begin{array}{l}\% \\
\text { PPM } \\
\text { PPM } \\
\text { PPM } \\
\text { PPM } \\
\% \\
\text { PPM } \\
\text { PPM } \\
\text { PPM } \\
\text { PPM } \\
\text { PPM } \\
\text { PPM } \\
\text { PPM } \\
z \\
\text { PPM } \\
\text { PPM } \\
\text { PPM } \\
\% \\
\text { PPM } \\
\text { PPM } \\
\text { PPM } \\
\% \\
\text { PPM } \\
\text { PPM } \\
\text { PPM } \\
\text { PPM }\end{array}$ & $\begin{array}{l}\text { 81GLA-A } \\
\text { 81GLA-A } \\
\text { 81GLA-A } \\
\text { 81GLA-A } \\
\text { 81GLA-A } \\
\text { 81GLA-A } \\
\text { 81GLA-A } \\
\text { 81GLA-A } \\
\text { 81GLA-A } \\
\text { 81GLA-A } \\
\text { 81GLA-A } \\
\text { 81GLA-A } \\
\text { 81GLA-A } \\
\text { 81GLA-A } \\
\text { 81GLA-A } \\
\text { 81GLA-A } \\
\text { 81GLA-A } \\
\text { 81GLA-A } \\
\text { 81GLA-A } \\
\text { 81GLA-A } \\
\text { 81GLA-A } \\
\text { 81GLA-A } \\
\text { 81GLA-A } \\
\text { 81GLA-A } \\
\text { 81GLA-A } \\
\text { 81GLA-A }\end{array}$ \\
\hline
\end{tabular}


TABLE A-II (cont)

\begin{tabular}{|c|c|c|c|c|c|}
\hline $\begin{array}{l}\text { SAMPLE } \\
\text { NUMBER }\end{array}$ & $\begin{array}{l}\text { CERTIF IED } \\
\text { VALUE } \\
\text { NAME }\end{array}$ & $\begin{array}{l}\text { CERTIFIED } \\
\text { VALUE }\end{array}$ & $\begin{array}{l}\text { CERTIFIED } \\
\text { VALUE } \\
\text { UNCERTAINTY }\end{array}$ & $\begin{array}{l}\text { CERT IF IED } \\
\text { VALUE } \\
\text { UNITS }\end{array}$ & REFERENCE \\
\hline \multirow{2}{*}{80.00612} & $\ldots .$. & $\cdots$ & - & $\cdots$ & n \\
\hline & $\begin{array}{l}R B \\
\text { SB } \\
S C \\
S I \\
S M \\
S R \\
T A \\
T B \\
T H \\
T I \\
T I \\
U \\
V \\
W \\
Y B \\
Z N \\
Z R\end{array}$ & $\begin{array}{c}77 . \\
2.1 \\
10 . \\
30.6 \\
4.9 \\
348 . \\
790 . \\
820 . \\
4.6 \\
3700 . \\
1.97 \\
60 . \\
610 . \\
2.8 \\
61 . \\
190 .\end{array}$ & $\begin{array}{c}9 . \\
.4 \\
1.5 \\
.5 \\
.5 \\
190 . \\
130 . \\
1.1 \\
170 . \\
.03 \\
5 . \\
30 . \\
.7 \\
4 . \\
20 .\end{array}$ & $\begin{array}{l}P P M \\
P P M \\
P P M \\
\% \\
\text { PPM } \\
\text { PPM } \\
P P B \\
\text { PPB } \\
\text { PPM } \\
\text { PPM } \\
\text { PPM } \\
\text { PPM } \\
\text { PPB } \\
\text { PPM } \\
\text { PPM } \\
\text { PPM }\end{array}$ & $\begin{array}{l}\text { 81GLA-A } \\
\text { 81GLA-A } \\
\text { 81GLA-A } \\
\text { 81GLA-A } \\
\text { 81GLA-A } \\
\text { 81GLA-A } \\
\text { 81GLA-A } \\
\text { 81GLA-A } \\
\text { 81GLA-A } \\
\text { 81GLA-A } \\
\text { 81GLA-A } \\
\text { 81GLA-A } \\
\text { 81GLA-A } \\
\text { 81GLA-A } \\
\text { 81GLA-A } \\
\text { 81GLA-A }\end{array}$ \\
\hline \multirow{2}{*}{80.00613} & & & & & \\
\hline & $\begin{array}{l}A G \\
A L \\
B \\
B A \\
B E \\
C \\
C A \\
C E \\
C L \\
C 0 \\
C R \\
C S \\
C U \\
E U \\
F \\
F E \\
G A \\
H F \\
K \\
L I \\
M G \\
M N \\
M 0 \\
N A \\
N B \\
N D \\
P \\
P B \\
R B \\
S B \\
S C \\
S I \\
S M \\
S R \\
T A\end{array}$ & $\begin{array}{c}109 . \\
7.29 \\
29 . \\
820 . \\
2.5 \\
39 . \\
8100 . \\
53 . \\
490 . \\
2 . \\
2.5 \\
9.9 \\
10.7 \\
770 . \\
360 . \\
1.31 \\
14 . \\
6.1 \\
3.59 \\
51 . \\
1700 . \\
282 . \\
2.3 \\
3 . \\
9 . \\
18.2 \\
205 . \\
22 . \\
156 . \\
1.29 \\
5 . \\
34 . \\
4.3 \\
114 . \\
.97\end{array}$ & $\begin{array}{c}9 . \\
.09 \\
2 . \\
40 . \\
.4 \\
10 . \\
600 . \\
6 . \\
40 . \\
.3 \\
.4 \\
.4 \\
.6 \\
120 . \\
40 . \\
.03 \\
2.0 \\
.5 \\
.08 \\
9 . \\
120 . \\
17 . \\
.6 \\
.1 \\
2 . \\
.8 \\
3 . \\
1 . \\
4 . \\
.14 \\
.8 \\
.6 \\
.4 \\
13 . \\
.11\end{array}$ & $\begin{array}{l}P P B \\
\% \\
P P M \\
P P M \\
P P M \\
P P M \\
P P M \\
P P M \\
P P M \\
P P M \\
P P M \\
P P M \\
P P M \\
P P B \\
P P M \\
\% \\
P P M \\
P P M \\
\% \\
P P M \\
P P M \\
P P M \\
P P M \\
\% \\
P P M \\
P P M \\
P P M \\
P P M \\
P P M \\
P P M \\
P P M \\
\% \\
P P M \\
? P M \\
P P M\end{array}$ & $\begin{array}{l}\text { 81GLA-A } \\
\text { 81GLA-A } \\
\text { 81GLA-A } \\
\text { 81GLA-A } \\
\text { 81GLA-A } \\
\text { 81GLA-A } \\
\text { 81GLA-A } \\
\text { 81GLA-A } \\
\text { 81GLA-A } \\
\text { 81GLA-A } \\
\text { 81GLA-A } \\
\text { 81GLA-A } \\
\text { 81GLA-A } \\
\text { 81GLA-A } \\
\text { 81GLA-A } \\
\text { 81GLA-A } \\
\text { 81GLA-A } \\
\text { 81GLA-A } \\
\text { 81GLA-A } \\
\text { 81GLA-A } \\
\text { 81GLA-A } \\
\text { 81GLA-A } \\
\text { 81GLA-A } \\
\text { 81GLA-A } \\
\text { 81GLA-A } \\
\text { 81GLA-A } \\
\text { 81GLA-A } \\
\text { 81GLA-A } \\
\text { 81GLA-A } \\
\text { 81GLA-A } \\
\text { 81GLA-A } \\
\text { 81GLA-A } \\
\text { 81GLA-A } \\
\text { 81GLA-A } \\
\text { 81GLA-A }\end{array}$ \\
\hline
\end{tabular}


TABLE A-II (cont)

\begin{tabular}{|c|c|c|c|c|c|}
\hline $\begin{array}{l}\text { SAMPLE } \\
\text { NUMBER }\end{array}$ & $\begin{array}{l}\text { CERTIFIED } \\
\text { VALUE } \\
\text { NAME }\end{array}$ & $\begin{array}{l}\text { CERTIFIED } \\
\text { VALUE }\end{array}$ & $\begin{array}{l}\text { CERTIFIED } \\
\text { VALUE } \\
\text { UNCERTAINTY }\end{array}$ & $\begin{array}{l}\text { CERTIFIED } \\
\text { VALUE } \\
\text { UNITS }\end{array}$ & REFERENCE \\
\hline .00613 & & & & & \\
\hline & $\begin{array}{l}\text { TH } \\
T I \\
U \\
V \\
W \\
Y B \\
Z N \\
Z R\end{array}$ & $\begin{array}{c}16 . \\
1600 . \\
5.84 \\
14 . \\
1.6 \\
2.9 \\
32 . \\
210 .\end{array}$ & $\begin{array}{c}2 . \\
200 . \\
1.07 \\
.1 \\
7.2 \\
10 .\end{array}$ & $\begin{array}{l}\text { PPM } \\
\text { PPM } \\
\text { PPM } \\
\text { PPM } \\
\text { PPM } \\
\text { PPM } \\
\text { PPM } \\
\text { PPM }\end{array}$ & $\begin{array}{l}\text { 81GLA-A } \\
\text { 81GLA-A } \\
\text { 81GLA-A } \\
\text { 81GLA-A } \\
\text { 81GLA-A } \\
\text { 81GLA-A } \\
\text { 81GLA-A } \\
\text { 81GLA-A }\end{array}$ \\
\hline
\end{tabular}

80.00614

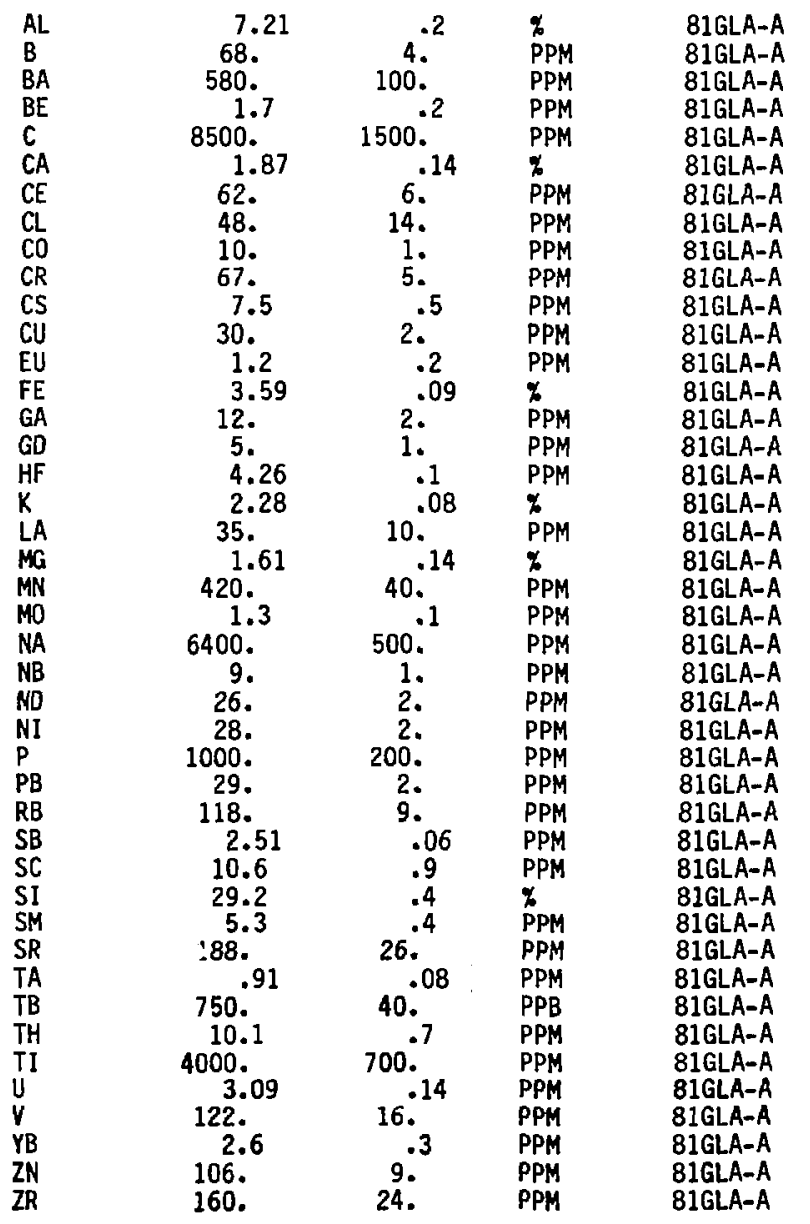




\begin{tabular}{|c|c|c|c|c|c|}
\hline 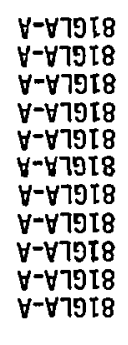 & $\begin{array}{l}\text { gdd } \\
\text { Wdd } \\
\text { Wdd } \\
\text { Wdd } \\
\text { Wdd } \\
\text { Wdd } \\
\text { Wdd } \\
\% \\
\text { Wdd } \\
\text { Wdd } \\
\%\end{array}$ & 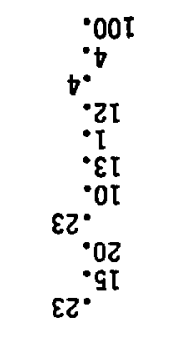 & $\begin{array}{c}\cdot 009 \\
\cdot 99 \\
z \cdot g \\
\cdot z \varepsilon \\
\cdot z \tau \\
\cdot 6 z \\
\cdot z t \\
2 I \cdot g \\
\cdot 0 z \varepsilon \\
\cdot z t \\
\varepsilon g \cdot \varepsilon\end{array}$ & 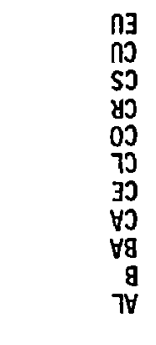 & $9 \tau 900^{\circ} 08$ \\
\hline 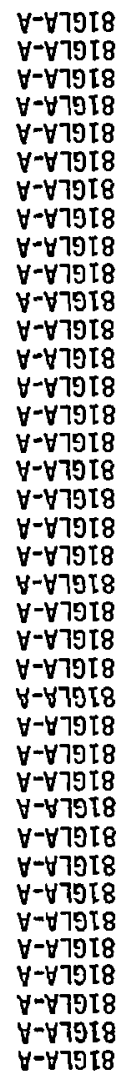 & $\begin{array}{l}\text { Wdd } \\
\text { Wdd } \\
\text { Wdd } \\
\text { Wdd } \\
\text { Wdd } \\
\text { Wdd } \\
\text { Wdd } \\
\text { Wdd } \\
\text { Wdd } \\
\text { Wdd } \\
\text { Wdd } \\
\text { \% } \\
\text { Wdd } \\
\text { Wdd } \\
\text { Wdd } \\
\text { Wdd } \\
\text { Wddd } \\
\text { Wdd } \\
\text { Wdd } \\
\text { X } \\
\text { Wdd } \\
\text { \% } \\
\% \\
\text { Wdd } \\
\text { Wdd } \\
\% \\
\text { Wdd } \\
\text { Wdd } \\
\text { Wdd } \\
\text { Wdd } \\
\text { Wdd } \\
\text { Wdd } \\
\text { Wdd } \\
\text { X } \\
\text { Wdd } \\
\text { Wdd } \\
\text { Wdd } \\
x\end{array}$ & 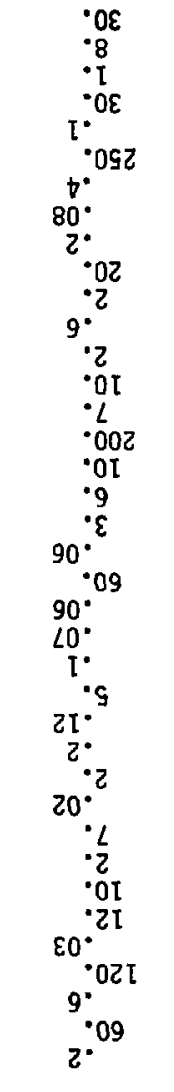 & 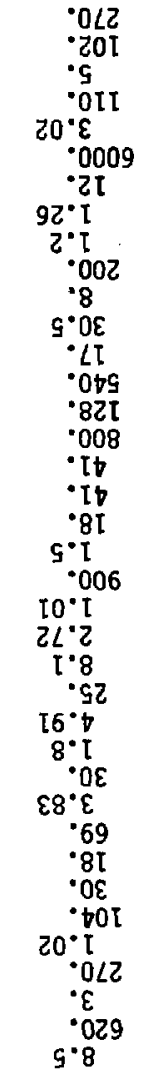 & 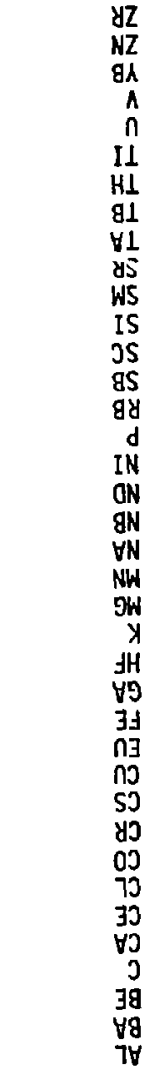 & $\mathrm{gtg} 00^{\circ} 08$ \\
\hline 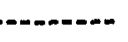 & & & 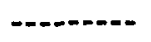 & 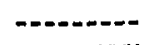 & \\
\hline 3y 35$]$ y & 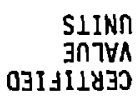 & $\begin{array}{r}A \perp N I \forall \perp y \exists J N n \\
3 \ldots 17 \forall A \\
031 \text { IIY33 }\end{array}$ & $\begin{array}{r}\exists \cap 7 \forall \wedge \\
0 \exists I J I \perp y \exists J\end{array}$ & $\begin{array}{r}\exists W \forall N \\
\exists \cap 7 \forall A \\
03 \text { IIIY3J }\end{array}$ & $\begin{array}{l}\text { YJgWnN } \\
\text { J7dWWS }\end{array}$ \\
\hline
\end{tabular}

(juos) II- 
TABLE A-II (cont)

\begin{tabular}{|c|c|c|c|c|c|}
\hline $\begin{array}{l}\text { SAMPLE } \\
\text { NUMBER }\end{array}$ & $\begin{array}{l}\text { CERT IF IED } \\
\text { VALUE } \\
\text { NAME }\end{array}$ & $\begin{array}{l}\text { CERTIFIED } \\
\text { VALUE }\end{array}$ & $\begin{array}{l}\text { CERTIFIED } \\
\text { VALUE } \\
\text { UNCERTAINTY }\end{array}$ & $\begin{array}{l}\text { CERTIFIED } \\
\text { VALUE } \\
\text { UNITS }\end{array}$ & REFERENCE \\
\hline .00616 & $\begin{array}{l}F E \\
G A \\
H F \\
K \\
M D \\
M G \\
M N \\
N A \\
N B \\
N B \\
N D \\
N I \\
P \\
P B \\
R B \\
S B \\
S C \\
S E \\
S I \\
S M \\
S R \\
T A \\
T B \\
T H \\
T I \\
U \\
Y \\
Y B \\
Z N \\
Z R\end{array}$ & $\begin{array}{c}2.07 \\
11 . \\
1.35 \\
1.43 \\
35.6 \\
2.8 \\
290 . \\
2.09 \\
5.27 \\
14 . \\
32 . \\
1330 . \\
38 . \\
85 . \\
3.3 \\
4.7 \\
3.4 \\
13.1 \\
2.7 \\
390 . \\
530 . \\
370 . \\
4.9 \\
1700 . \\
5.57 \\
128 . \\
1.1 \\
80 . \\
56 .\end{array}$ & $\begin{array}{c}2.14 \\
.04 \\
.13 \\
.5 \\
.22 \\
30 . \\
.14 \\
.06 \\
3 . \\
3 . \\
140 . \\
5 . \\
7 . \\
.4 \\
.6 \\
.3 \\
.1 \\
.3 \\
60 . \\
30 . \\
40 . \\
.02 \\
300 . \\
.15 \\
8 . \\
.2 \\
9 . \\
5 .\end{array}$ & $\begin{array}{l}\% \\
\text { PPM } \\
\text { PPM } \\
\% \\
\text { PPM } \\
\% \\
\text { PPM } \\
\% \\
\text { PPM } \\
\text { PPM } \\
\text { PPM } \\
\text { PPM } \\
\text { PPM } \\
\text { PPM } \\
\text { PPM } \\
\text { PPM } \\
\text { PPM } \\
\% \\
\text { PPM } \\
\text { PPM } \\
\text { PPB } \\
\text { PPB } \\
\text { PPM } \\
\text { PPM } \\
\text { PPM } \\
\text { PPM } \\
\text { PPM } \\
\text { PPM } \\
\text { PPM }\end{array}$ & $\begin{array}{l}\text { 81GLA-A } \\
\text { 81GLA-A } \\
\text { 81GLA-A } \\
\text { 81GLA-A } \\
\text { 81GLA-A } \\
\text { 81GLA-A } \\
\text { 81GLA-A } \\
\text { 81GLA-A } \\
\text { 81GLA-A } \\
\text { 81GLA-A } \\
\text { 81GLA-A } \\
\text { 81GLA-A } \\
\text { 81GLA-A } \\
\text { 81GLA-A } \\
\text { 81GLA-A } \\
\text { 81GLA-A } \\
\text { 81GLA-A } \\
\text { 81GLA-A } \\
\text { 81GLA-A } \\
\text { 81GLA-A } \\
\text { 81GLA-A } \\
\text { 81GLA-A } \\
\text { 81GLA-A } \\
\text { 81GLA-A } \\
\text { 81GLA-A } \\
\text { 81GLA-A } \\
\text { 81GLA-A } \\
\text { 81GLA-A } \\
\text { 81GLA-A }\end{array}$ \\
\hline
\end{tabular}

80.00617

\begin{tabular}{|c|c|c|c|c|}
\hline $\begin{array}{l}A L \\
B A \\
B E \\
C \\
C A \\
C L \\
C R \\
C S \\
C U \\
E U \\
F \\
F E \\
G A \\
G D \\
H F \\
K \\
L A \\
L I \\
M D \\
M G \\
M N \\
N A\end{array}$ & $\begin{array}{c}9.79 \\
590 . \\
B .9 \\
80 . \\
7900 . \\
440 . \\
2.6 \\
1.52 \\
4 . \\
3.6 \\
930 . \\
3.64 \\
37 . \\
12 . \\
27 . \\
3.57 \\
170 . \\
29 . \\
5.3 \\
550 . \\
1720 . \\
6.61\end{array}$ & $\begin{array}{c}20.12 \\
.2 \\
20 . \\
600 . \\
30 . \\
1.1 \\
.08 \\
2 . \\
.4 \\
60 . \\
.14 \\
1 . \\
3 . \\
.06 \\
30 . \\
7 . \\
100 . \\
140 . \\
.14\end{array}$ & $\begin{array}{l}\% \\
\text { PPM } \\
\text { PPM } \\
\text { PPM } \\
\text { PPM } \\
\text { PPM } \\
\text { PPM } \\
\text { PPM } \\
\text { PPM } \\
\text { PPM } \\
\text { PPM } \\
\% \\
\text { PPM } \\
\text { PPM } \\
\text { PPM } \\
\% \\
\text { PPM } \\
\text { PPM } \\
\text { PPM } \\
\text { PPM } \\
\text { PPM } \\
\%\end{array}$ & $\begin{array}{l}\text { 81GLA-A } \\
\text { 81GLA-A } \\
\text { 81GLA-A } \\
\text { 81GLA-A } \\
\text { 81GLA-A } \\
\text { 81GLA-A } \\
\text { 81GLA-A } \\
\text { 81GLA-A } \\
\text { 81GLA-A } \\
\text { 81GLA-A } \\
\text { 81GLA-A } \\
\text { 81GLA-A } \\
\text { 81GLA-A } \\
\text { 81GLA-A } \\
\text { 81GLA-A } \\
\text { 81GLA-A } \\
\text { 81GLA-A } \\
\text { 81GLA-A } \\
\text { 81GLA-A } \\
\text { 81GLA-A } \\
\text { 81GLA-A } \\
\text { 81GLA-A }\end{array}$ \\
\hline
\end{tabular}


TABLE A-II (cont)

\begin{tabular}{|c|c|c|c|c|c|}
\hline $\begin{array}{l}\text { SAMPLEE } \\
\text { NUMBER }\end{array}$ & $\begin{array}{l}\text { CERTIFIED } \\
\text { VALUE } \\
\text { NAME }\end{array}$ & $\begin{array}{l}\text { CERTIFIED } \\
\text { VALUE }\end{array}$ & $\begin{array}{l}\text { CERTIFIED } \\
\text { VALUE } \\
\text { UNCERTAINTY }\end{array}$ & $\begin{array}{l}\text { CERT IFIED } \\
\text { VALUE } \\
\text { UNITS }\end{array}$ & REFERENCE \\
\hline 80.00617 & $\cdots$ & - & $\cdots$ & -..n....... & n......... \\
\hline & $\begin{array}{l}N B \\
N D \\
N I \\
P \\
P B \\
R B \\
S B \\
S C \\
S I \\
S M \\
S N \\
S R \\
T A \\
T B \\
T H \\
T I \\
U \\
W \\
Y B \\
Z N \\
Z R\end{array}$ & $\begin{array}{c}270 . \\
82 . \\
2.1 \\
710 . \\
17 . \\
120 . \\
1.65 \\
678 . \\
27.8 \\
15 . \\
10 . \\
730 . \\
19 . \\
1.7 \\
33 . \\
910 . \\
9.07 \\
3.9 \\
4.4 \\
230 . \\
1260 .\end{array}$ & $\begin{array}{c}20 . \\
5 . \\
20.6 \\
1 . \\
6 . \\
.03 \\
2 . \\
.4 \\
3 . \\
30 . \\
30 . \\
2.3 \\
5 . \\
60 . \\
.07 \\
.2 \\
30.4 \\
80 .\end{array}$ & $\begin{array}{l}\text { PPM } \\
P P M \\
P P M \\
P P M \\
P P M \\
P P M \\
P P M \\
P P B \\
z \\
P P M \\
P P M \\
P P M \\
P P M \\
P P M \\
P P M \\
P P M \\
P P M \\
P P M \\
P P M \\
P P M \\
P P M\end{array}$ & $\begin{array}{l}\text { 81GLA-A } \\
\text { 81GLA-A } \\
\text { 81GLA-A } \\
\text { 81GLA-A } \\
\text { 81GLA-A } \\
\text { 81GLA-A } \\
\text { 81GLA-A } \\
\text { 81GLA-A } \\
\text { 81GLA-A } \\
\text { 81GLA-A } \\
\text { 81GLA-A } \\
\text { 81GLA-A } \\
\text { 81GLA-A } \\
\text { 81GLA-A } \\
\text { 81GLA-A } \\
\text { 81GLA-A } \\
\text { 81GLA-A } \\
\text { 81GLA-A } \\
\text { 81GLA-A } \\
\text { 81GLA-A } \\
\text { 81GLA-A }\end{array}$ \\
\hline 80.00618 & $F$ & 7.14 & .48 & PPM & $75 N B S-C$ \\
\hline 80.00619 & U & 105. & 5. & PPB & $79 H-8$ \\
\hline 80.00620 & NA-22 & 289. & 3. & $\mathrm{NCI} / \mathrm{L}$ & 64NBS-B \\
\hline 80.00621 & NA-22 & 289. & 3. & $N C I / L$ & 64NBS-B \\
\hline 80.00622 & $\mathrm{H}-3$ & & 300. & PCI/L & $79 H-8$ \\
\hline 80.00623 & NA-22 & 576. & 6. & $P C I / G$ & 64NBS-B \\
\hline 80.00624 & NA-22 & 576. & 6. & $\mathrm{PCl} / \mathrm{G}$ & 64NBS-B \\
\hline 80.00625 & $N A-22$ & 576. & 6. & $P C I / G$ & 64NBS-B \\
\hline 80.00626 & $N A-22$ & 576. & 6. & $P C I / G$ & 64NBS-B \\
\hline 80.00627 & NA-22 & 576. & 6. & $\mathrm{PCI} / \mathrm{G}$ & 64NBS-B \\
\hline
\end{tabular}


TABLE A-II (cont)

\begin{tabular}{|c|c|c|c|c|c|}
\hline $\begin{array}{l}\text { SAMPLE } \\
\text { NUMBER }\end{array}$ & $\begin{array}{l}\text { CERTIFIED } \\
\text { VALUE } \\
\text { NAME }\end{array}$ & $\begin{array}{l}\text { CERTIFIED } \\
\text { VALUE }\end{array}$ & $\begin{array}{l}\text { CERTIFIED } \\
\text { VALUE } \\
\text { UNCERTAINTY }\end{array}$ & $\begin{array}{l}\text { CERTIFIED } \\
\text { VALUE } \\
\text { UNITS }\end{array}$ & REFERENCE \\
\hline \multirow[t]{2}{*}{80.00628} & 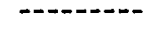 & $-\ldots . .-$ & ------- & $-\cdots$ & --- \\
\hline & NA-22 & 576. & 6. & $\mathrm{PCI} / \mathrm{G}$ & 64NBS-B \\
\hline \multicolumn{6}{|l|}{80.00629} \\
\hline \multicolumn{6}{|c|}{80.00630} \\
\hline & $\begin{array}{l}\text { AS } \\
\text { BR } \\
C A \\
C D \\
C U \\
F E \\
H G \\
K \\
M N \\
M 0 \\
N A \\
N I \\
S E \\
Z N\end{array}$ & $\begin{array}{c}6 . \\
9 . \\
190 . \\
32 . \\
2 . \\
18.3 \\
1 . \\
1360 . \\
8.5 \\
400 . \\
8 . \\
180 . \\
1.1 \\
10.6\end{array}$ & $\begin{array}{r}10 . \\
7 . \\
.30 \\
.80 \\
40 . \\
.5 \\
1.5 \\
.2 \\
1 .\end{array}$ & $\begin{array}{l}\text { PPB } \\
\text { PPM } \\
\text { PPM } \\
\text { PPB } \\
\text { PPM } \\
\text { PPM } \\
\text { PPB } \\
\text { PPM } \\
\text { PPM } \\
\text { PPB } \\
\text { PPM } \\
\text { PPB } \\
\text { PPM } \\
\text { PPM }\end{array}$ & $\begin{array}{l}\text { 78NBS-E } \\
\text { 78NBS-E } \\
\text { 78NBS-E } \\
\text { 78NBS-E } \\
\text { 78NBS-E } \\
\text { 78NBS-E } \\
\text { 78NBS-E } \\
\text { 78NBS-E } \\
\text { 78NBS-E } \\
\text { 7ONBS-E } \\
\text { 78NBS-E } \\
\text { 78NBS-E } \\
\text { 78NBS-E } \\
\text { 78NBS-E }\end{array}$ \\
\hline \multicolumn{6}{|l|}{80.00631} \\
\hline & $\begin{array}{l}A S \\
B R \\
C A \\
C D \\
C O \\
C U \\
F E \\
H G \\
K \\
M N \\
M O \\
N A \\
N I \\
R B \\
S E \\
Z N\end{array}$ & $\begin{array}{c}410 . \\
1 . \\
140 . \\
29 . \\
20 . \\
2.2 \\
8.7 \\
6 . \\
1120 . \\
20.1 \\
1.6 \\
6 . \\
160 . \\
7 . \\
400 . \\
19.4\end{array}$ & $\begin{array}{r}50 . \\
20 . \\
4 . \\
10 . \\
.3 \\
.6 \\
.7 \\
20 . \\
.4 \\
1.5 \\
100 . \\
1 .\end{array}$ & $\begin{array}{l}\text { PPB } \\
\text { PPM } \\
\text { PPM } \\
\text { PPB } \\
\text { PPB } \\
\text { PPM } \\
\text { PPM } \\
\text { PPB } \\
\text { PPM } \\
\text { PPM } \\
\text { PPM } \\
\text { PPM } \\
\text { PPB } \\
\text { PPM } \\
\text { PPB } \\
\text { PPM }\end{array}$ & $\begin{array}{l}78 \mathrm{NBS}-\mathrm{F} \\
78 \mathrm{NBS}-\mathrm{F} \\
78 \mathrm{NBS}-\mathrm{F} \\
\text { 78NBS-F } \\
\text { 78NBS-F } \\
\text { 78NBS-F } \\
\text { 78NBS-F } \\
\text { 78NBS-F } \\
\text { 78NBS-F } \\
\text { 78NBS-F } \\
\text { 78NBS-F } \\
\text { 78NBS-F } \\
\text { 78NBS-F } \\
\text { 78NBS-F } \\
\text { 78NBS-F } \\
\text { 78NBS-F }\end{array}$ \\
\hline \multicolumn{6}{|c|}{80.00632} \\
\hline & $\begin{array}{l}\text { CR } \\
U\end{array}$ & $\begin{array}{l}2.12 \\
470 .\end{array}$ & $30 . .05$ & $\begin{array}{l}\text { PPM } \\
\text { PPB }\end{array}$ & $\begin{array}{l}\text { 76NBS-D } \\
\text { 80GLA-C }\end{array}$ \\
\hline \multicolumn{6}{|c|}{80.00633} \\
\hline & $\begin{array}{l}\text { AL. } \\
A S \\
B \\
B A \\
B E \\
C A \\
C D \\
C E \\
C O\end{array}$ & $\begin{array}{c}14 . \\
145 . \\
39.2 \\
1500 . \\
12 . \\
1.11 \\
1 . \\
180 . \\
46 .\end{array}$ & $\begin{array}{l}.01 \\
.15\end{array}$ & $\begin{array}{l}\% \\
\text { PPM } \\
\text { PPM } \\
\text { PPM } \\
\text { PPM } \\
\varnothing \\
\text { PPM } \\
\text { PPM } \\
\text { PPM }\end{array}$ & $\begin{array}{l}\text { 79NBS-B } \\
79 \mathrm{NBS}-\mathrm{B} \\
806 \mathrm{GL}-\mathrm{A} \\
79 \mathrm{NBS}-\mathrm{B} \\
79 \mathrm{NBS}-\mathrm{B} \\
79 \mathrm{NBS}-\mathrm{B} \\
79 \mathrm{NBS}-\mathrm{B} \\
79 \mathrm{NBS}-\mathrm{B} \\
79 \mathrm{NBS}-\mathrm{B}\end{array}$ \\
\hline
\end{tabular}


TABLE A-II (cont)

\begin{tabular}{|c|c|c|c|c|c|}
\hline $\begin{array}{l}\text { SAMPLE } \\
\text { NUMBER }\end{array}$ & $\begin{array}{l}\text { CERTIFIED } \\
\text { VALUE } \\
\text { NAME }\end{array}$ & $\begin{array}{l}\text { CERTIFIED } \\
\text { VALUE }\end{array}$ & $\begin{array}{l}\text { CERTIF IED } \\
\text { VALUE } \\
\text { UNCERTAINTY }\end{array}$ & $\begin{array}{l}\text { CERTIFIED } \\
\text { VALUE } \\
\text { UNITS }\end{array}$ & REFERENCE \\
\hline 80.00633 & --- & $\cdots-$ & $\ldots$ & $\cdots$ & 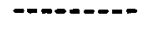 \\
\hline & 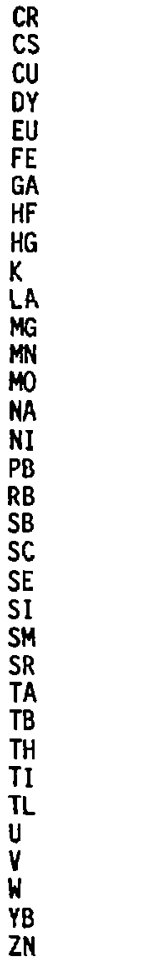 & $\begin{array}{c}196 . \\
11 . \\
118 . \\
14 . \\
4 . \\
9.4 \\
58.4 \\
7.6 \\
160 . \\
1.88 \\
84 . \\
4550 . \\
190 . \\
29 . \\
1700 . \\
127 . \\
72.4 \\
131 . \\
7 . \\
40 . \\
10.3 \\
22.8 \\
16 . \\
830 . \\
2.1 \\
2.9 \\
24.7 \\
8000 . \\
5.7 \\
10.2 \\
300 . \\
5.4 \\
8 . \\
220 .\end{array}$ & $\begin{array}{c}6 . \\
3 . \\
.1 \\
10 . \\
.06 \\
6 . \\
100 . \\
100 . \\
4 . \\
.4 \\
2 . \\
.6 \\
.8 \\
30 . \\
.2 \\
.1 \\
.3 \\
.2 \\
.1 \\
.4 \\
10 .\end{array}$ & $\begin{array}{l}\text { PPM } \\
\text { PPM } \\
\text { PPM } \\
\text { PPM } \\
\text { PPM } \\
\text { \% } \\
\text { PPM } \\
\text { PPM } \\
\text { PPB } \\
\text { \% } \\
\text { PPM } \\
\text { PPM } \\
\text { PPM } \\
\text { PPH } \\
\text { PPM } \\
\text { PPM } \\
\text { PPM } \\
\text { PPM } \\
\text { PPM } \\
\text { PPM } \\
\text { PPM } \\
\text { X } \\
\text { PPM } \\
\text { PPM } \\
\text { PPM } \\
\text { PPM } \\
\text { PPM } \\
\text { PPM } \\
\text { PPM } \\
\text { PPM } \\
\text { PPM } \\
\text { PPM } \\
\text { PPM } \\
\text { PPM }\end{array}$ & $\begin{array}{l}79 N B S-B \\
79 N B S-B \\
79 N B S-B \\
80 G L A-D \\
79 N B S-B \\
79 N B S-B \\
79 N B S-B \\
79 N B S-B \\
79 N B S-B \\
79 N B S-B \\
\text { 80GLA-D } \\
79 N B S-B \\
79 N B S-B \\
79 N B S-B \\
\text { 79NBS-B } \\
\text { 79NBS-B } \\
\text { 79NBS-B } \\
\text { 79NBS-B } \\
\text { 79NBSS-B } \\
\text { 79NBSS-B } \\
\text { 79NBS-B } \\
\text { 79NBS-B } \\
\text { 80GLA-A } \\
\text { 79NBS-B } \\
\text { 80GLA-D } \\
\text { 80GLA-D } \\
\text { 79NBS-B } \\
\text { 79NBS-B } \\
\text { 79NBS-B } \\
\text { 79NBS-B } \\
\text { 79NBS-B } \\
\text { 80GLA-D } \\
80 G L A-D \\
\text { 79NBS-B }\end{array}$ \\
\hline 80.00634 & & & & & \\
\hline & $S$ & 5460 & 30. & PPM & 74NBS-G \\
\hline 80.00635 & $S$ & 2.016 & .014 & $x$ & 74NBS-G \\
\hline 80.00636 & $S$ & 3.02 & .008 & $\%$ & 74NBS-G \\
\hline 80.00637 & CS-137 & 1020 & & $\mathrm{PCl} / \mathrm{L}$ & 80EML-A \\
\hline 80.00638 & CS-137 & 1020 & & $\mathrm{PCI} / \mathrm{L}$ & BOEML-A \\
\hline 80.00639 & CS-137 & 1020. & & $\mathrm{PCI} / \mathrm{L}$ & 80ERL-A \\
\hline
\end{tabular}


TABLE A-II (cont)

\begin{tabular}{|c|c|c|c|c|c|}
\hline $\begin{array}{l}\text { SAMPLE } \\
\text { NUMBER }\end{array}$ & $\begin{array}{l}\text { CERTIFIED } \\
\text { VALUE } \\
\text { NAME }\end{array}$ & $\begin{array}{l}\text { CERTIFIED } \\
\text { VALUE }\end{array}$ & $\begin{array}{l}\text { CERTIF IED } \\
\text { VALUE } \\
\text { UNCERTAINTY }\end{array}$ & $\begin{array}{l}\text { CERTIFIED } \\
\text { VALUE } \\
\text { UNITS }\end{array}$ & REFERENCE \\
\hline --..-. & -......... & -...-n-n & -...- & -.......- & - \\
\hline 80.00640 & CS-137 & .72 & & $\mathrm{PCI} / \mathrm{G}$ & 80EML $-A$ \\
\hline 80.00641 & CS-137 & .72 & & PCI/G & 80EML-A \\
\hline 80.00642 & CS-137 & .72 & & $\mathrm{PCI} / \mathrm{G}$ & 80EML-A \\
\hline 80.00643 & $\begin{array}{l}A G \\
S B \\
T L\end{array}$ & $\begin{array}{r}1.2 \\
8.2 \\
25.2\end{array}$ & & $\begin{array}{l}\text { PPB } \\
\text { PPB } \\
\text { PPB }\end{array}$ & $\begin{array}{l}\text { 79EPA-A } \\
\text { 79EPA-A } \\
\text { 79EPA-A }\end{array}$ \\
\hline 80.00644 & $\begin{array}{l}A G \\
S B \\
T L\end{array}$ & $\begin{array}{r}4.9 \\
97.5 \\
6.3\end{array}$ & & $\begin{array}{l}\text { PPB } \\
\text { PPB } \\
\text { PPB }\end{array}$ & $\begin{array}{l}\text { 79EPA-A } \\
\text { 79EPA-A } \\
\text { 79EPA-A }\end{array}$ \\
\hline 80.00645 & $\begin{array}{l}\text { NH4 } \\
\text { N03 } \\
\text { PO4 }\end{array}$ & $\begin{array}{l}.19 \\
.31 \\
.031\end{array}$ & & $\begin{array}{l}\text { PPM } \\
\text { PPM } \\
\text { PPM }\end{array}$ & $\begin{array}{l}\text { 78EPA-A } \\
\text { 78EPA-A } \\
\text { 78EPA-A }\end{array}$ \\
\hline 80.00646 & $\begin{array}{l}\text { NH4 } \\
\text { NO3 } \\
\text { P04 }\end{array}$ & $\begin{array}{l}1.3 \\
1.59 \\
.154\end{array}$ & & $\begin{array}{l}\text { PPM } \\
\text { PPM } \\
\text { PPM }\end{array}$ & $\begin{array}{l}\text { 78EPA-A } \\
\text { 78EPA-A } \\
\text { 78EPA-A }\end{array}$ \\
\hline 80.00647 & $\begin{array}{l}\mathbf{N} \\
\text { P04 }\end{array}$ & $\begin{array}{r}.52 \\
.14\end{array}$ & & $\begin{array}{l}\text { PPM } \\
\text { PPM }\end{array}$ & $\begin{array}{l}\text { 78EPA-A } \\
\text { 78EPA-A }\end{array}$ \\
\hline 80.00648 & $\begin{array}{l}\text { N } \\
\text { PO4 }\end{array}$ & $\begin{array}{r}4.12 \\
.93\end{array}$ & & $\begin{array}{l}\text { PPM } \\
\text { PPM }\end{array}$ & $\begin{array}{l}\text { 78EPA-A } \\
\text { 78EPA-A }\end{array}$ \\
\hline 80.00649 & $\begin{array}{l}A L \\
A S \\
B E \\
C D \\
C D \\
C R \\
C U \\
F E \\
H G \\
M N \\
N I \\
P B \\
S E \\
V \\
Z N\end{array}$ & $\begin{array}{c}61 . \\
24 . \\
24 . \\
6.5 \\
30.5 \\
4.4 \\
8.7 \\
16 . \\
.4 \\
7.9 \\
8.7 \\
30 . \\
8.7 \\
78.7 \\
6.1\end{array}$ & & $\begin{array}{l}P P \\
P P B \\
P P B \\
P P B \\
P P B \\
P P B \\
P P B \\
P P B \\
P P B \\
P P E \\
P P B \\
P P B \\
P P B \\
P P B \\
P P B\end{array}$ & $\begin{array}{l}\text { 76EPA-A } \\
\text { 76EPA-A } \\
\text { 76EPA-A } \\
\text { 76EPA-A } \\
\text { 76EPA-A } \\
\text { 76EPA-A } \\
\text { 76EPA-A } \\
\text { 76EPA-A } \\
\text { 76EPA-A } \\
\text { 76EPA-A } \\
\text { 76EPA-A } \\
\text { 76EPA-A } \\
\text { 76EPA-A } \\
\text { 76EPA-A } \\
\text { 76EPA-A }\end{array}$ \\
\hline
\end{tabular}


TABLE A-II (cont)

\begin{tabular}{|c|c|c|c|c|c|}
\hline $\begin{array}{l}\text { SAMPLE } \\
\text { NUMBER }\end{array}$ & $\begin{array}{l}\text { CERTIFIED } \\
\text { VALUE } \\
\text { NAME }\end{array}$ & $\begin{array}{l}\text { CERTIFIED } \\
\text { VALUE }\end{array}$ & $\begin{array}{l}\text { CERTIFIED } \\
\text { VALUE } \\
\text { UNCERTAINTY }\end{array}$ & $\begin{array}{l}\text { CERT IFIED } \\
\text { VALUE } \\
\text { UNITS }\end{array}$ & REFERENCE \\
\hline -...-n-n & $\cdots-\cdots$ & --.--- & - n......... & - & -..non- \\
\hline 80.00650 & $\begin{array}{l}A L \\
A S \\
B E \\
C D \\
C O \\
C R \\
C U \\
F E \\
H G \\
M N \\
N I \\
P B \\
S E \\
V \\
Z N\end{array}$ & $\begin{array}{l}852 . \\
182 . \\
261 . \\
59 . \\
348 . \\
304 . \\
374 . \\
796 . \\
7.6 \\
478 . \\
165 . \\
383 . \\
48 . \\
848 . \\
478 .\end{array}$ & & $\begin{array}{l}\text { PPB } \\
\text { PPB } \\
\text { PPB } \\
\text { PPB } \\
\text { PPB } \\
\text { PPB } \\
\text { PPB } \\
\text { PPB } \\
\text { PPB } \\
\text { PPB } \\
\text { PPB } \\
\text { PPB } \\
\text { PPB } \\
\text { PPB } \\
\text { PPB }\end{array}$ & $\begin{array}{l}\text { 76EPA-A } \\
\text { 76EPA-A } \\
\text { 76EPA-A } \\
\text { 76EPA-A } \\
\text { 76EPA-A } \\
\text { 76EPA-A } \\
\text { 76EPA-A } \\
\text { 76EPA-A } \\
\text { 76EPA-A } \\
\text { 76EPA-A } \\
\text { 76EPA-A } \\
\text { 76EPA-A } \\
\text { 76EPA-A } \\
\text { 76EPA-A } \\
\text { 76EPA-A }\end{array}$ \\
\hline 80.00651 & $\begin{array}{l}A L \\
A S \\
B E \\
C D \\
C D \\
C R \\
C U \\
F E \\
H G \\
M N \\
N I \\
P B \\
S E \\
Y \\
Z N\end{array}$ & $\begin{array}{c}435 . \\
61 . \\
183 . \\
27 . \\
96 . \\
65 . \\
37 . \\
78 . \\
4.4 \\
47 . \\
96 . \\
113 . \\
16 . \\
470 . \\
26 .\end{array}$ & & $\begin{array}{l}\text { PPB } \\
P P B \\
P P B \\
P P B \\
P P B \\
P P B \\
\text { PPB } \\
\text { PPB } \\
\text { PPB } \\
\text { PPB } \\
\text { PPB } \\
\text { PPB } \\
\text { PPB } \\
\text { PPB } \\
\text { PPB }\end{array}$ & $\begin{array}{l}\text { 76EPA-A } \\
\text { 76EPA-A } \\
\text { 76EPA-A } \\
\text { 76EPA-A } \\
\text { 76EPA-A } \\
\text { 76EPA-A } \\
\text { 76EPA-A } \\
\text { 76EPA-A } \\
\text { 76EPA-A } \\
\text { 76EPA-A } \\
\text { 76EPA-A } \\
\text { 76EPA-A } \\
\text { 76EPA-A } \\
\text { 76EPA-A } \\
\text { 76EPA-A }\end{array}$ \\
\hline 80.00652 & $\begin{array}{l}C A \\
C L \\
\text { COND } \\
F \\
\text { HARD } \\
K \\
\text { MG } \\
\text { NA } \\
\text { SO4 } \\
\text { TDS }\end{array}$ & $\begin{array}{c}40.6 \\
87.9 \\
572 . \\
1.3 \\
136 . \\
9.8 \\
8.4 \\
46.6 \\
93.6 \\
338 .\end{array}$ & & $\begin{array}{l}\text { PPM } \\
\text { PPM } \\
\text { UMHO* } \\
\text { PPM } \\
\text { MG/L } \\
\text { PPM } \\
\text { PPM } \\
\text { PPM } \\
\text { PPM } \\
\text { PPM }\end{array}$ & $\begin{array}{l}\text { 78EPA-B } \\
\text { 78EPA-B } \\
\text { 78EPA-B } \\
\text { 78EPA-B } \\
\text { 78EPA-B } \\
\text { 78EPA-B } \\
\text { 78EPA-B } \\
\text { 78EPA-B } \\
\text { 78EPA-B } \\
\text { 78EPA-B }\end{array}$ \\
\hline 80.00653 & $\begin{array}{l}\text { CA } \\
C L \\
\text { COND } \\
\text { F } \\
\text { HARD }\end{array}$ & $\begin{array}{c}5.3 \\
18.4 \\
113 . \\
.43 \\
20.7\end{array}$ & & $\begin{array}{l}\mathrm{PPM} \\
\mathrm{PPM} \\
\text { UMHO*} \\
\text { PPM } \\
\text { MG/L }\end{array}$ & $\begin{array}{l}\text { 78EPA-B } \\
\text { 78EPA-B } \\
\text { 78EPA-B } \\
\text { 78EPA-B } \\
\text { 78EPA-B }\end{array}$ \\
\hline
\end{tabular}


TABLE A-II (cont)

\begin{tabular}{|c|c|c|c|c|c|}
\hline $\begin{array}{l}\text { SAMPLE } \\
\text { NUMBER }\end{array}$ & $\begin{array}{l}\text { CERTIFIED } \\
\text { VALUE } \\
\text { NAME }\end{array}$ & $\begin{array}{l}\text { CERTIFIED } \\
\text { VALUE }\end{array}$ & $\begin{array}{l}\text { CERTIFIED } \\
\text { VALUE } \\
\text { UNCERTAINTY }\end{array}$ & $\begin{array}{l}\text { CERTIFIED } \\
\text { VALUE } \\
\text { UNITS }\end{array}$ & REFERENCE \\
\hline .00653 & $\begin{array}{l}K \\
M G \\
N A \\
\text { SO4 } \\
\text { TDS }\end{array}$ & $\begin{array}{r}2.1 \\
1.8 \\
8.2 \\
7.2 \\
54 .\end{array}$ & & $\begin{array}{l}\text { PPM } \\
\text { PPM } \\
\text { PPM } \\
\text { PPM } \\
\text { PPM }\end{array}$ & $\begin{array}{l}\text { 73EPA-B } \\
\text { 78EPA-B } \\
\text { 78EPA-B } \\
\text { 7BEPA-B } \\
\text { 78EPA-B }\end{array}$ \\
\hline 80.00654 & $\begin{array}{l}A G \\
A S \\
B A \\
C D \\
C R \\
H G \\
P B \\
S E\end{array}$ & $\begin{array}{c}24 . \\
22 . \\
142 . \\
2.9 \\
11 . \\
1.4 \\
22 . \\
6 .\end{array}$ & & $\begin{array}{l}\text { PPB } \\
P P B \\
P P B \\
P P B \\
P P B \\
P P B \\
P P B \\
P P B\end{array}$ & $\begin{array}{l}\text { 78EPA-B } \\
\text { 78EPA-B } \\
78 E P A-B \\
\text { 78EPA-B } \\
\text { 78EPA-B } \\
\text { 78EPA-B } \\
\text { 78EPA-B } \\
\text { 78EPA-B }\end{array}$ \\
\hline 80.00655 & $\begin{array}{l}A G \\
A S \\
B A \\
C D \\
C R \\
H G \\
P B \\
S E\end{array}$ & $\begin{array}{c}24 . \\
40 . \\
335 . \\
4.3 \\
42 . \\
4 . \\
38 . \\
7.3\end{array}$ & & $\begin{array}{l}P P B \\
P P B \\
P P B \\
P P B \\
P P B \\
P P B \\
P P B \\
P P B\end{array}$ & $\begin{array}{l}\text { 78EPA-B } \\
\text { 78EPA-B } \\
\text { 78EPA-B } \\
\text { 78EPA-B } \\
\text { 78EPA-B } \\
\text { 78EPA-B } \\
\text { 78EPA-B } \\
\text { 7BEPA-B }\end{array}$ \\
\hline$B 0.00656$ & U & 150. & 15. & PPB & $\mathrm{BOH}-8$ \\
\hline 80.00660 & AS & 4. & & $U G / S$ & ??EPA-A \\
\hline 80.00661 & AS & 112. & & $U G / S$ & ??EPA-A \\
\hline 80.00662 & AS & 23. & & $U G / S$ & ??EPA-A \\
\hline 80.00663 & AS & 62. & & $U G / S$ & ??EPA-A \\
\hline 80.00664 & AS & 38. & & $U G / S$ & ??EPA-A \\
\hline 80.00665 & AS & 79. & & $U G / S$ & ??EPA-A \\
\hline 80.00666 & AS & 18. & & $U G / S$ & ??EPA-A \\
\hline 80.00667 & AS & 9. & & UG/S & ??EPA-A \\
\hline
\end{tabular}


TABLE A-II (cont)

\begin{tabular}{|c|c|c|c|c|c|}
\hline $\begin{array}{l}\text { SAMPLE } \\
\text { NUMBER }\end{array}$ & $\begin{array}{l}\text { CERTIF IED } \\
\text { YALUE } \\
\text { NAME } \\
-\end{array}$ & $\begin{array}{l}\text { CERTIFIED } \\
\text { VALUE }\end{array}$ & $\begin{array}{l}\text { CERTIFIED } \\
\text { YALUE } \\
\text { UNCERTAINTY }\end{array}$ & $\begin{array}{l}\text { CERTIFIED } \\
\text { VALUE } \\
\text { UNITS }\end{array}$ & REFERENCE \\
\hline 80.00668 & AS & 145. & & $U G / S$ & ??EPA-A \\
\hline 80.00669 & AS & & & UG/S & ??EPA-A \\
\hline 80.00570 & $\begin{array}{l}\mathrm{NO3} \\
\text { S04 }\end{array}$ & $\begin{array}{l}200 . \\
150 .\end{array}$ & & $\begin{array}{l}U G / S \\
U G / S\end{array}$ & $\begin{array}{l}\text { ??EPA-B } \\
\text { ??EPA-B }\end{array}$ \\
\hline 80.00671 & $\begin{array}{l}\text { N03 } \\
\text { S04 }\end{array}$ & $\begin{array}{l}100 . \\
300 \text {. }\end{array}$ & & $\begin{array}{l}\text { UG/S } \\
U G / S\end{array}$ & $\begin{array}{l}\text { ??EPA-B } \\
\text { ??EPA-B }\end{array}$ \\
\hline 80.00672 & $\begin{array}{l}\mathrm{NO3} \\
\mathrm{SO4}\end{array}$ & $\begin{array}{l}300 . \\
450 .\end{array}$ & & $\begin{array}{l}U G / S \\
U G / S\end{array}$ & $\begin{array}{l}\text { ??EPA-B } \\
\text { ??EPA-B }\end{array}$ \\
\hline 80.00673 & $\begin{array}{l}\text { NO3 } \\
\text { S04 }\end{array}$ & & & $\begin{array}{l}U G / S \\
U G / S\end{array}$ & $\begin{array}{l}\text { ??EPA-B } \\
\text { ??EPA-B }\end{array}$ \\
\hline 80.00674 & $\begin{array}{l}\text { N03 } \\
\text { SO4 }\end{array}$ & $\begin{array}{l}900 . \\
2000 \text {. }\end{array}$ & & $\begin{array}{l}\text { UG/S } \\
U G / S\end{array}$ & $\begin{array}{l}\text { ??EPA-B } \\
\text { ??EPA-B }\end{array}$ \\
\hline 80.00675 & $\begin{array}{l}\mathrm{NO3} \\
\text { SO4 }\end{array}$ & $\begin{array}{l}1300 \\
2500 \text {. }\end{array}$ & & $\begin{array}{l}U G / S \\
U G / S\end{array}$ & $\begin{array}{l}\text { ??EPA-B } \\
\text { ??EPA-B }\end{array}$ \\
\hline 80.00676 & $\begin{array}{l}\mathrm{NO3} \\
\mathrm{SO4}\end{array}$ & $\begin{array}{l}1100 . \\
3000 \text {. }\end{array}$ & & $\begin{array}{l}\text { UG/S } \\
\text { UG/S }\end{array}$ & $\begin{array}{l}\text { ??EPA-B } \\
\text { ??EPA-B }\end{array}$ \\
\hline 80.00677 & $\begin{array}{l}\mathrm{NO3} \\
\text { SO4 }\end{array}$ & $\begin{array}{l}2000 \\
4000\end{array}$ & & $\begin{array}{l}\text { UG/S } \\
\text { UG/S }\end{array}$ & $\begin{array}{l}\text { ??EPA-B } \\
\text { 3?EРA-B }\end{array}$ \\
\hline 80.00678 & $\begin{array}{l}\mathrm{N03} \\
\text { S04 }\end{array}$ & $\begin{array}{l}1800 . \\
5000 .\end{array}$ & & $\begin{array}{l}U G / S \\
U G / S\end{array}$ & $\begin{array}{l}\text { ??EPA-B } \\
\text { ??EPA-B }\end{array}$ \\
\hline 80.00679 & $\begin{array}{l}\mathrm{N03} \\
\mathrm{SO4}\end{array}$ & $\begin{array}{l}1600 \\
6000\end{array}$ & & $\begin{array}{l}\text { UG/S } \\
\text { UG/S }\end{array}$ & $\begin{array}{l}\text { ??EPA-B } \\
\text { ??EPA-B }\end{array}$ \\
\hline 80.00680 & PB & 100. & & $U G / S$ & ??EPA-C \\
\hline 0681 & PB & 250. & & UG/S & ??EPA-C \\
\hline
\end{tabular}




\section{TABLE A-II (cont)}

\begin{tabular}{|c|c|c|c|c|c|}
\hline $\begin{array}{l}\text { SAMPLE } \\
\text { NUMBER }\end{array}$ & $\begin{array}{l}\text { CERTIFIED } \\
\text { VALUE } \\
\text { NAME }\end{array}$ & $\begin{array}{l}\text { CERT IFIED } \\
\text { VALUE }\end{array}$ & $\begin{array}{l}\text { CERTIFIED } \\
\text { VALUE } \\
\text { UNCERTAINTY }\end{array}$ & $\begin{array}{l}\text { CERTIFIED } \\
\text { VRLUE } \\
\text { UNITS }\end{array}$ & REFERENCE \\
\hline 80.00682 & PB & 400 & 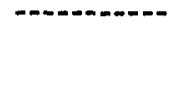 & UG/S & ??EPA-C \\
\hline 80.00683 & PB & 800 . & & UG/S & ??EPA-C \\
\hline 80.00684 & PB & 1000 & & UG/S & ??EPA-C \\
\hline 80.00685 & PB & 1200. & & $U G / S$ & ??EPA-C \\
\hline 80.00686 & PB & & & $\mathrm{UG} / \mathrm{S}$ & ??EPA-C \\
\hline 80.00687 & PB & 1800. & & $U G / S$ & ??EPA-C \\
\hline 80.00688 & PB & 2000 & & $U G / S$ & ??EPA-C \\
\hline .0068 & PB & 2400. & & UG/S & ??EPA-C \\
\hline
\end{tabular}


APPENDIX B

CONTROL CHARTS FOR STABLE ELEMENT QA PROGRAM 

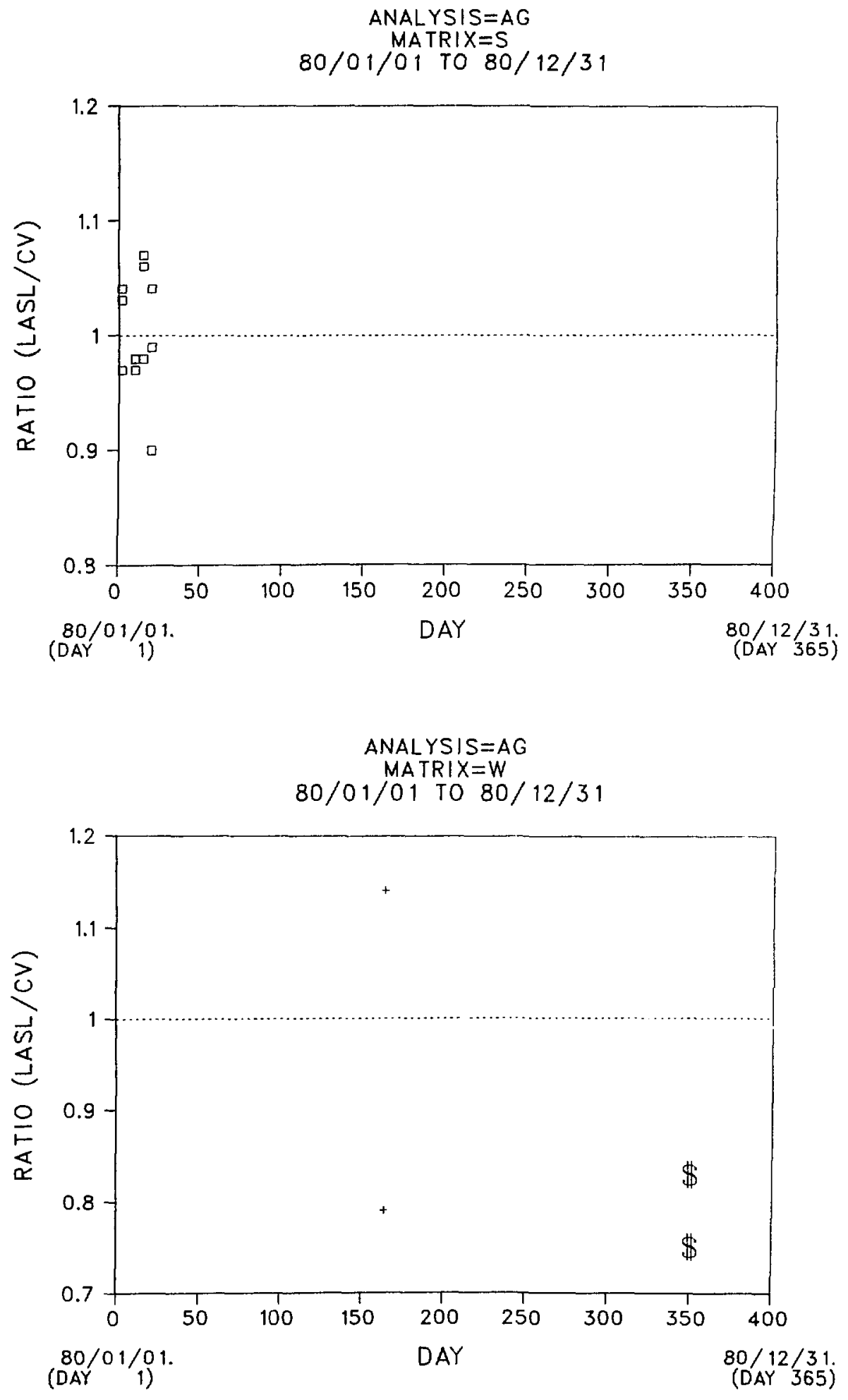

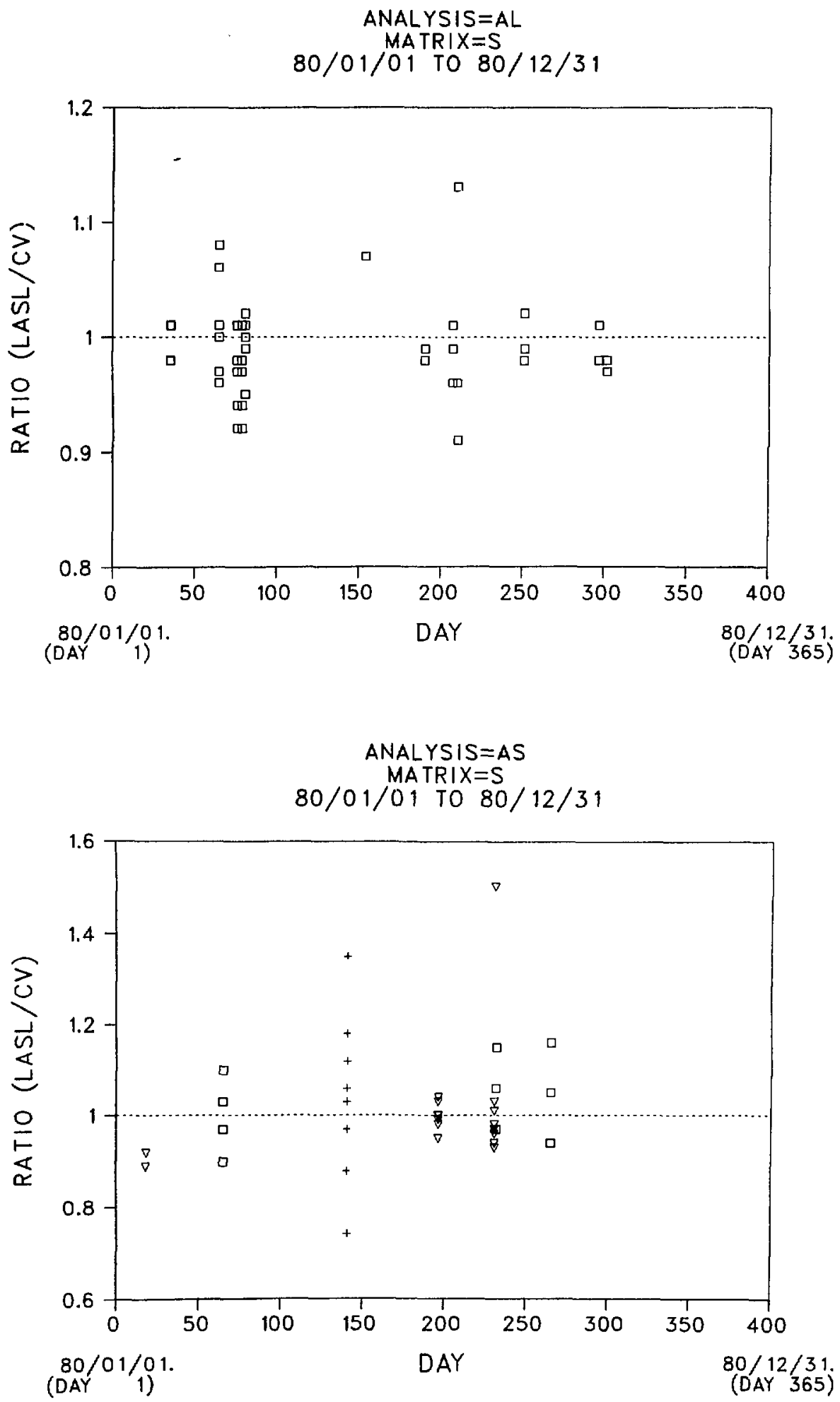

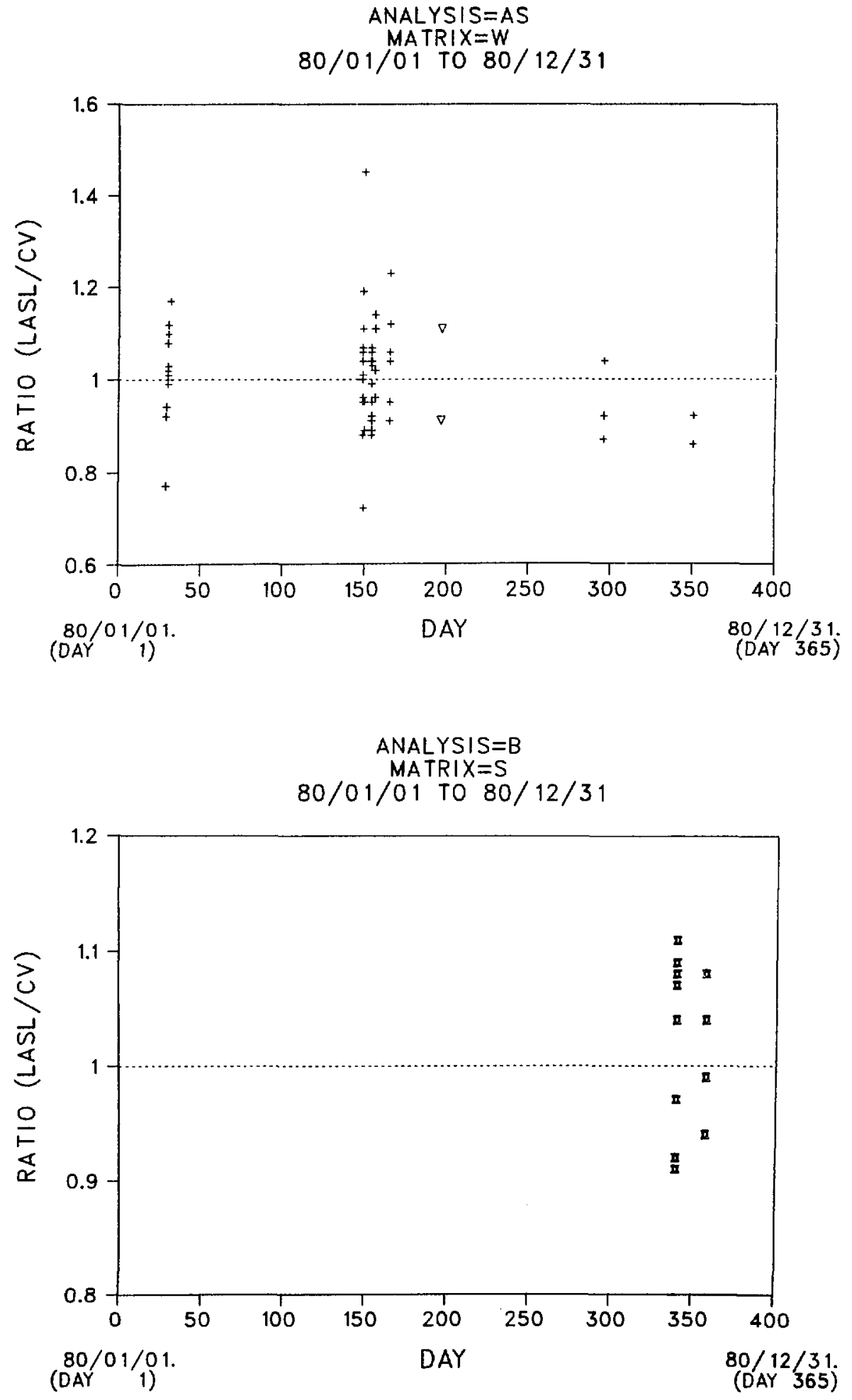

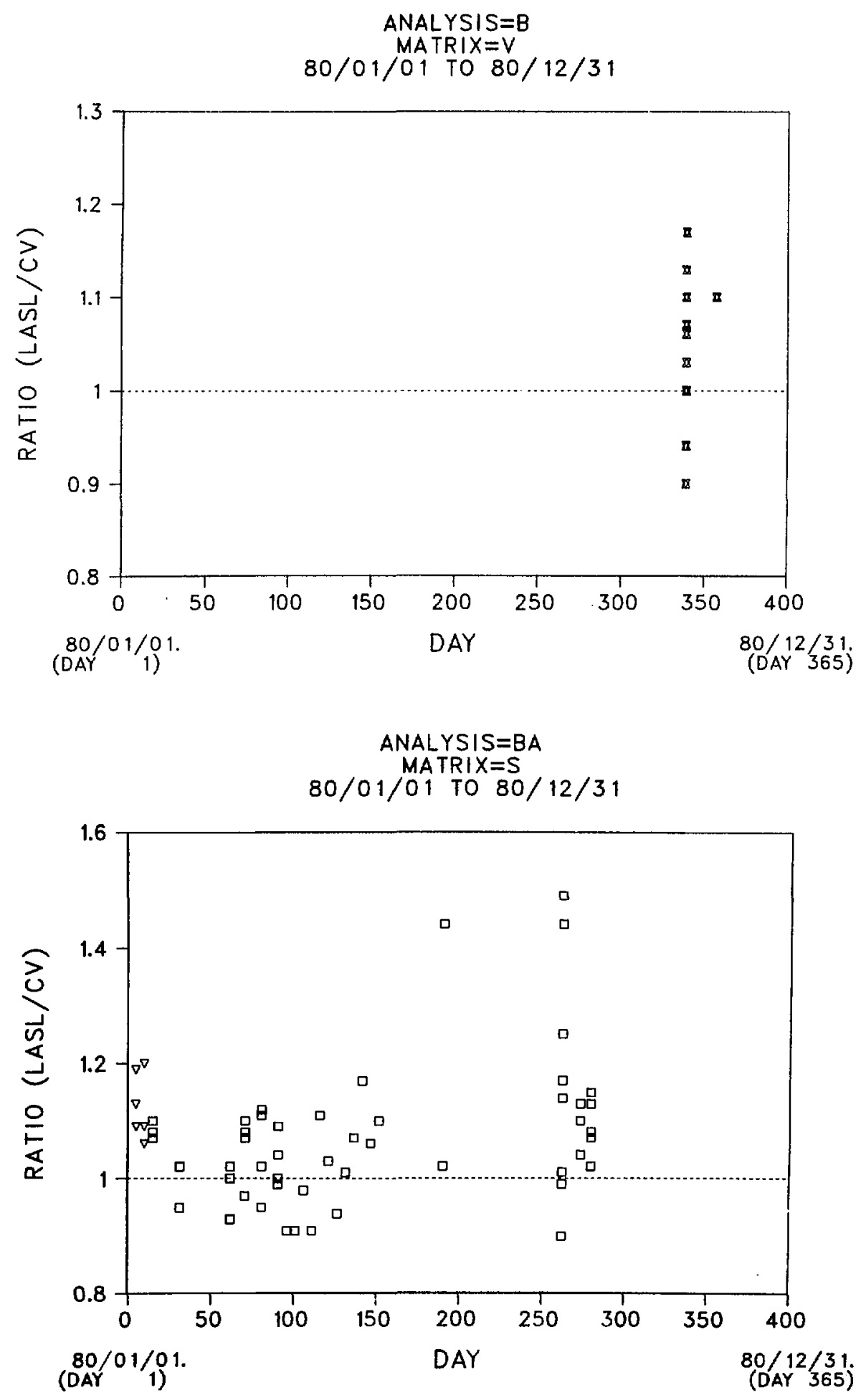

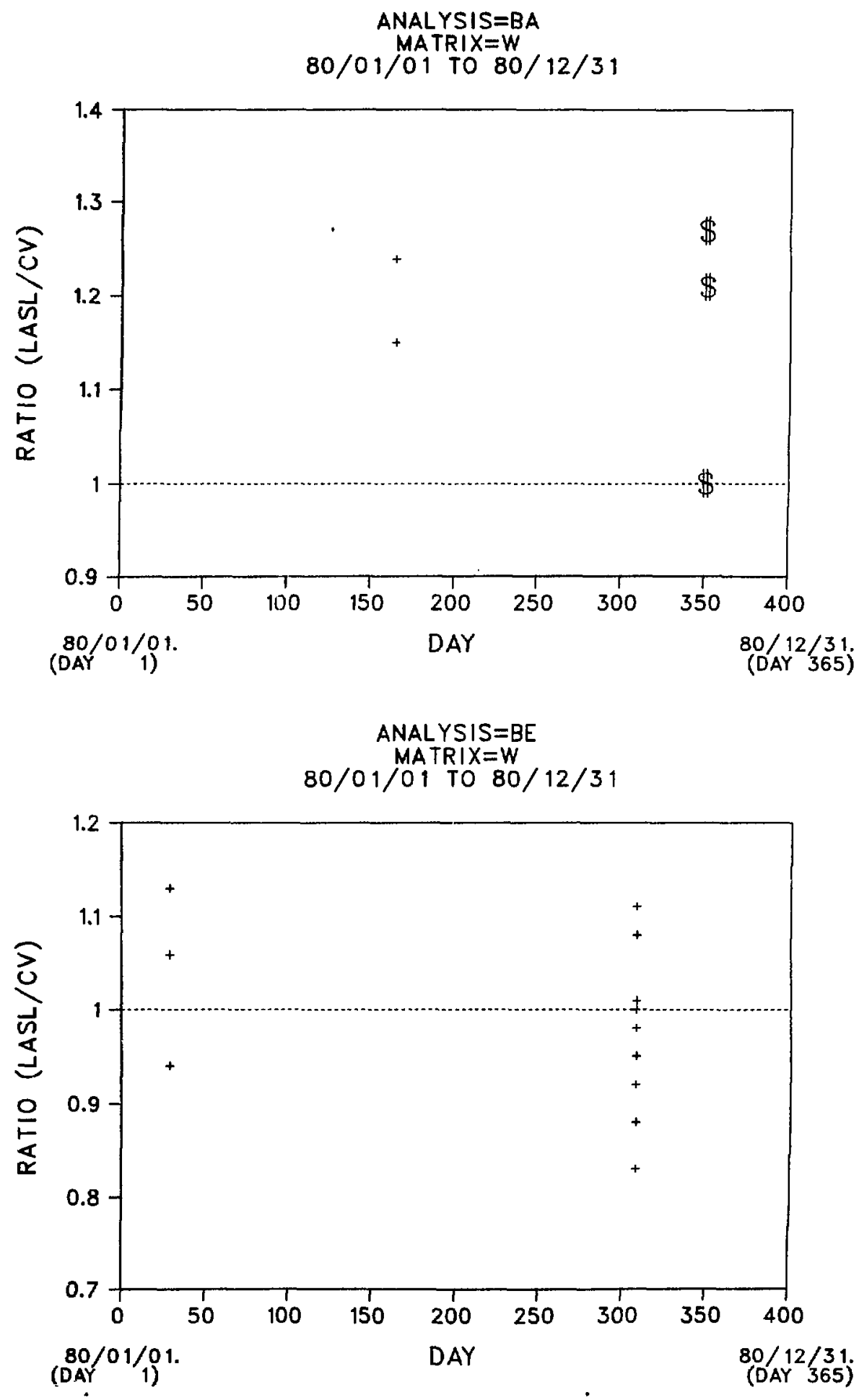

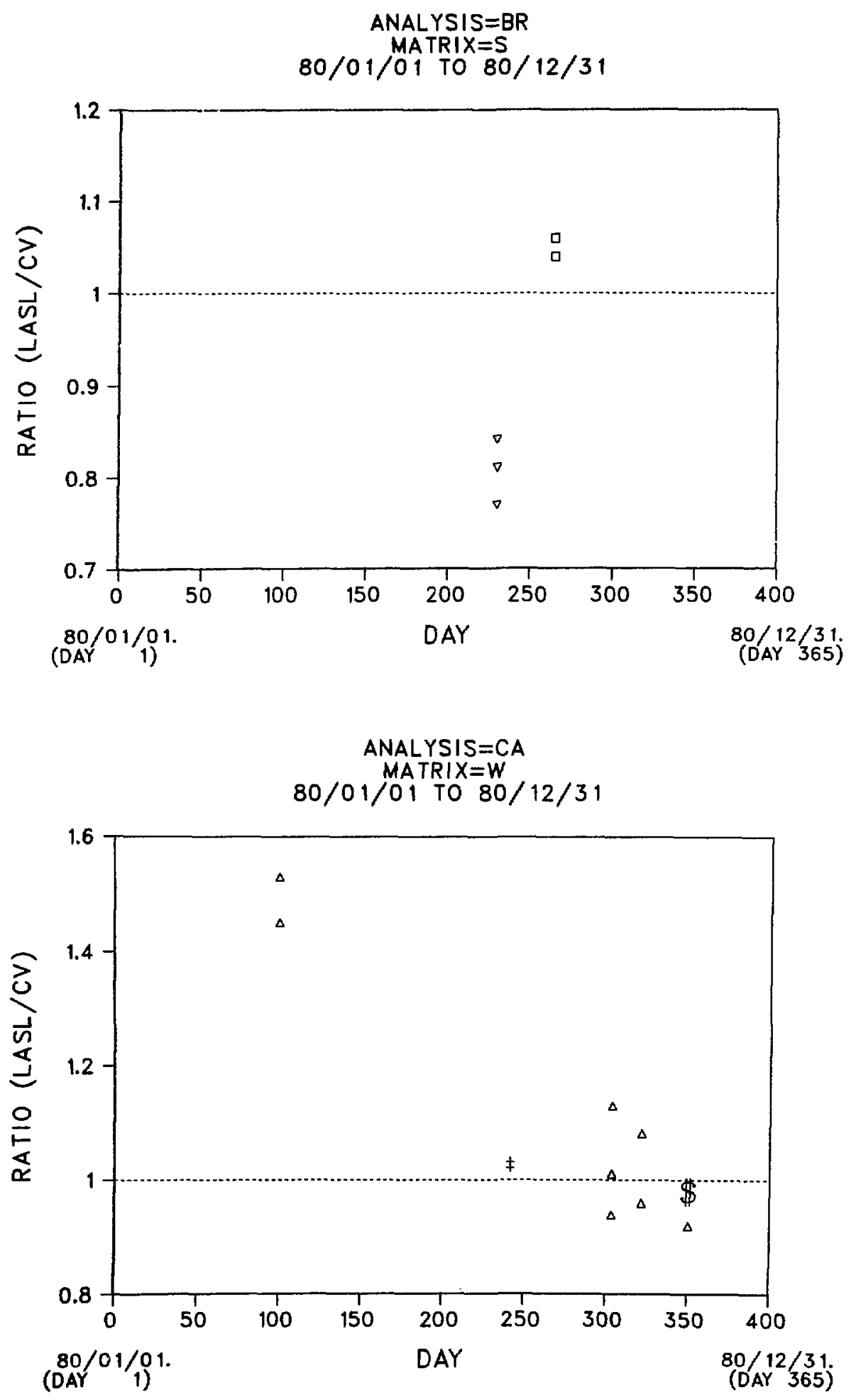

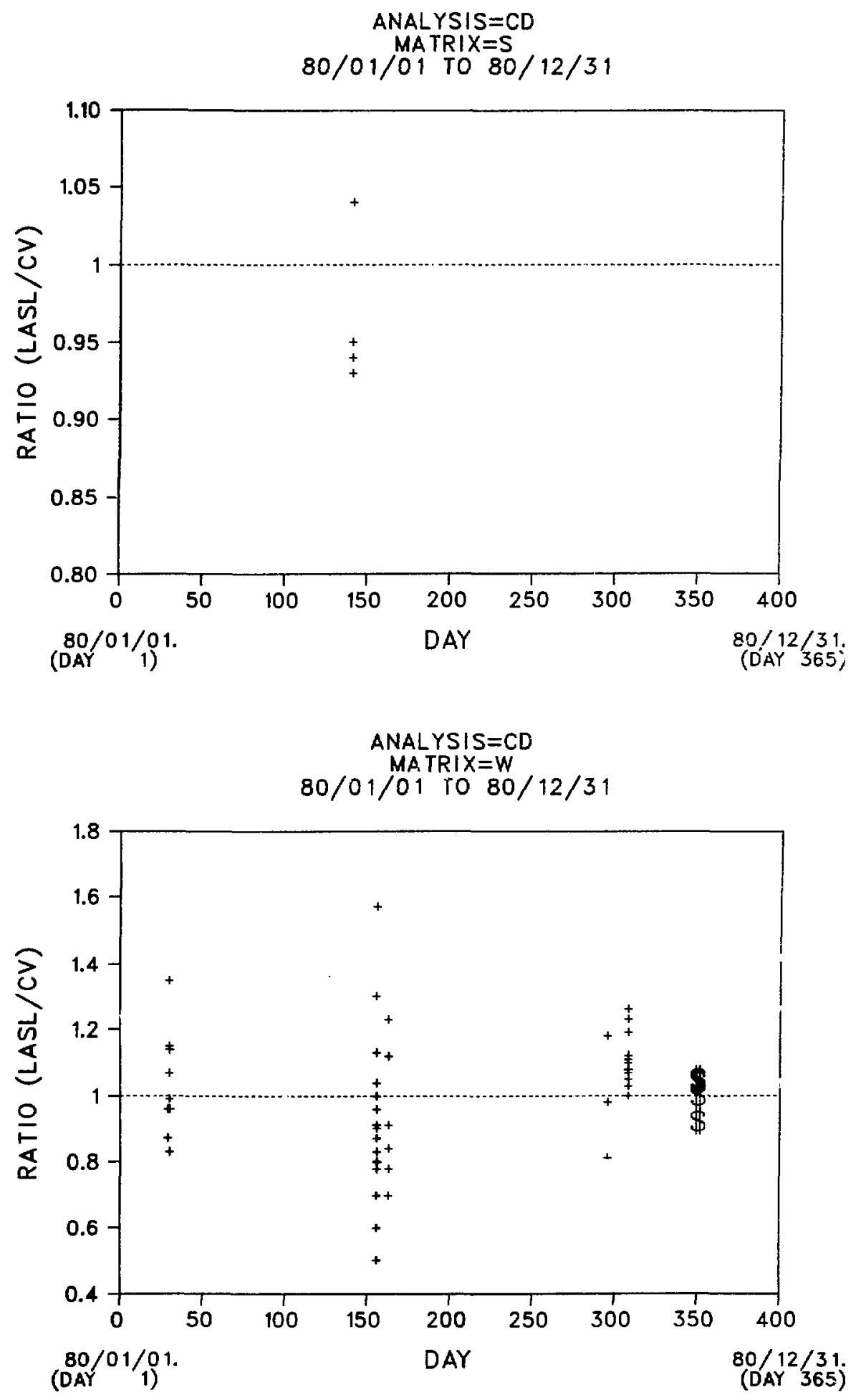

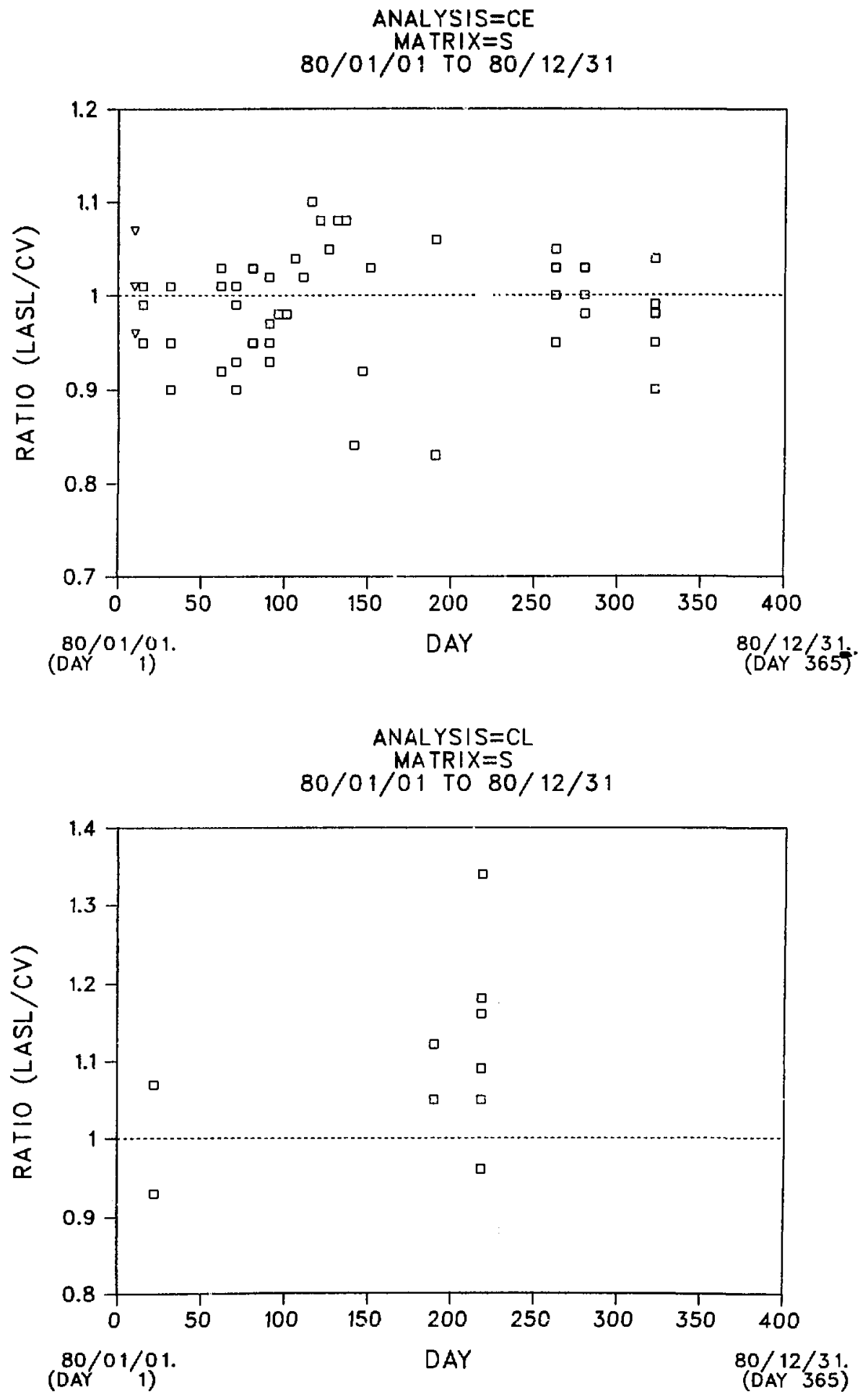

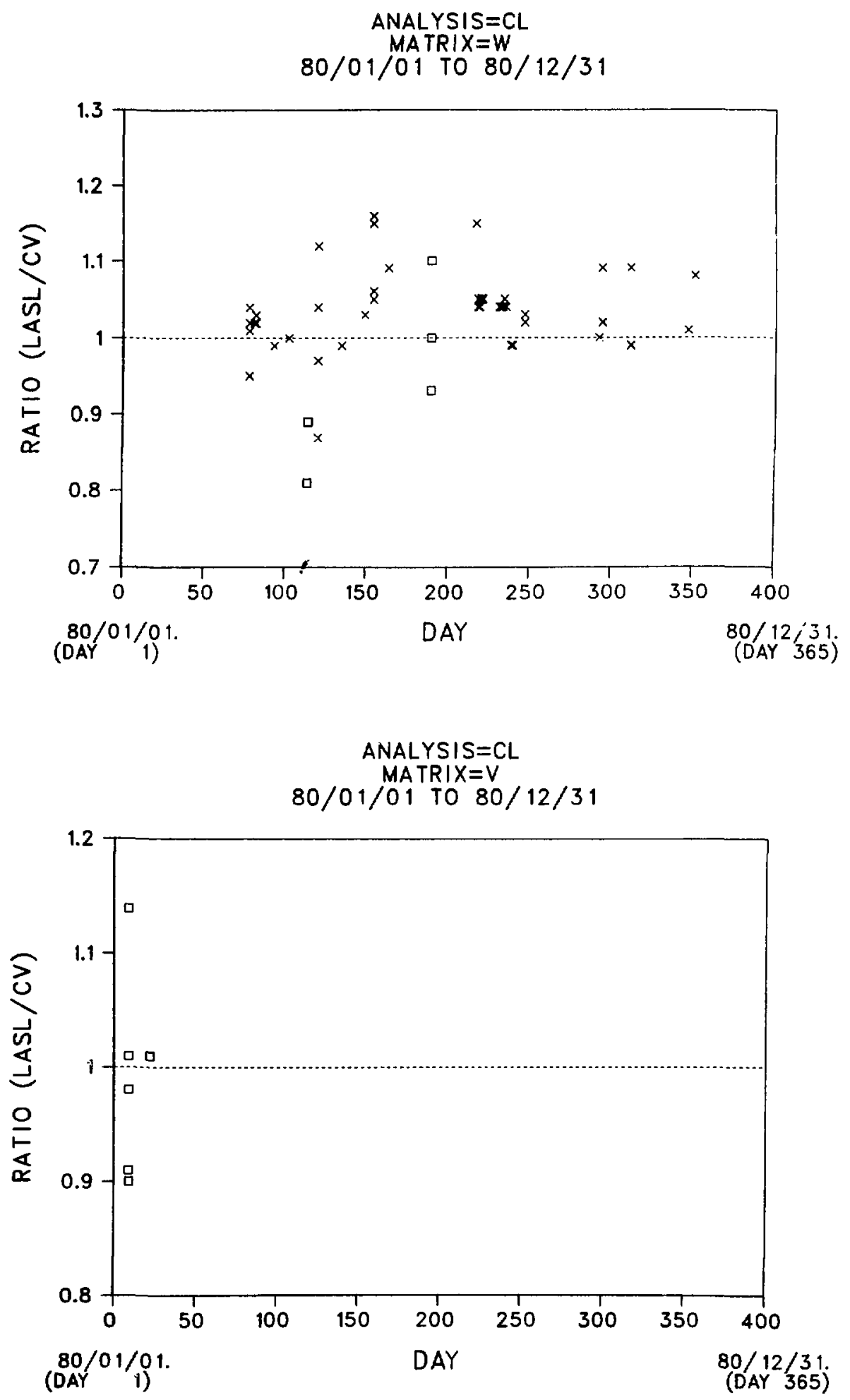

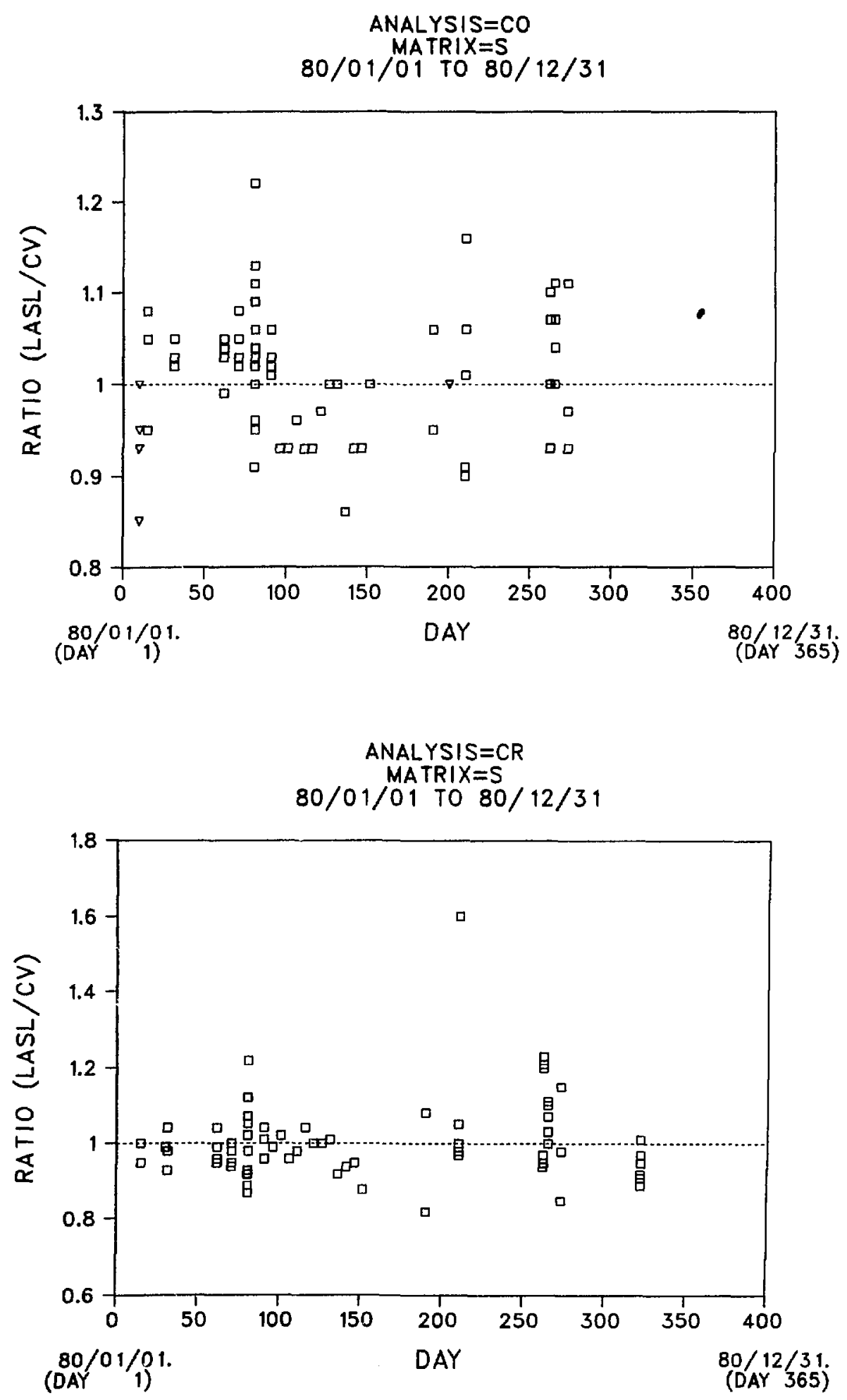

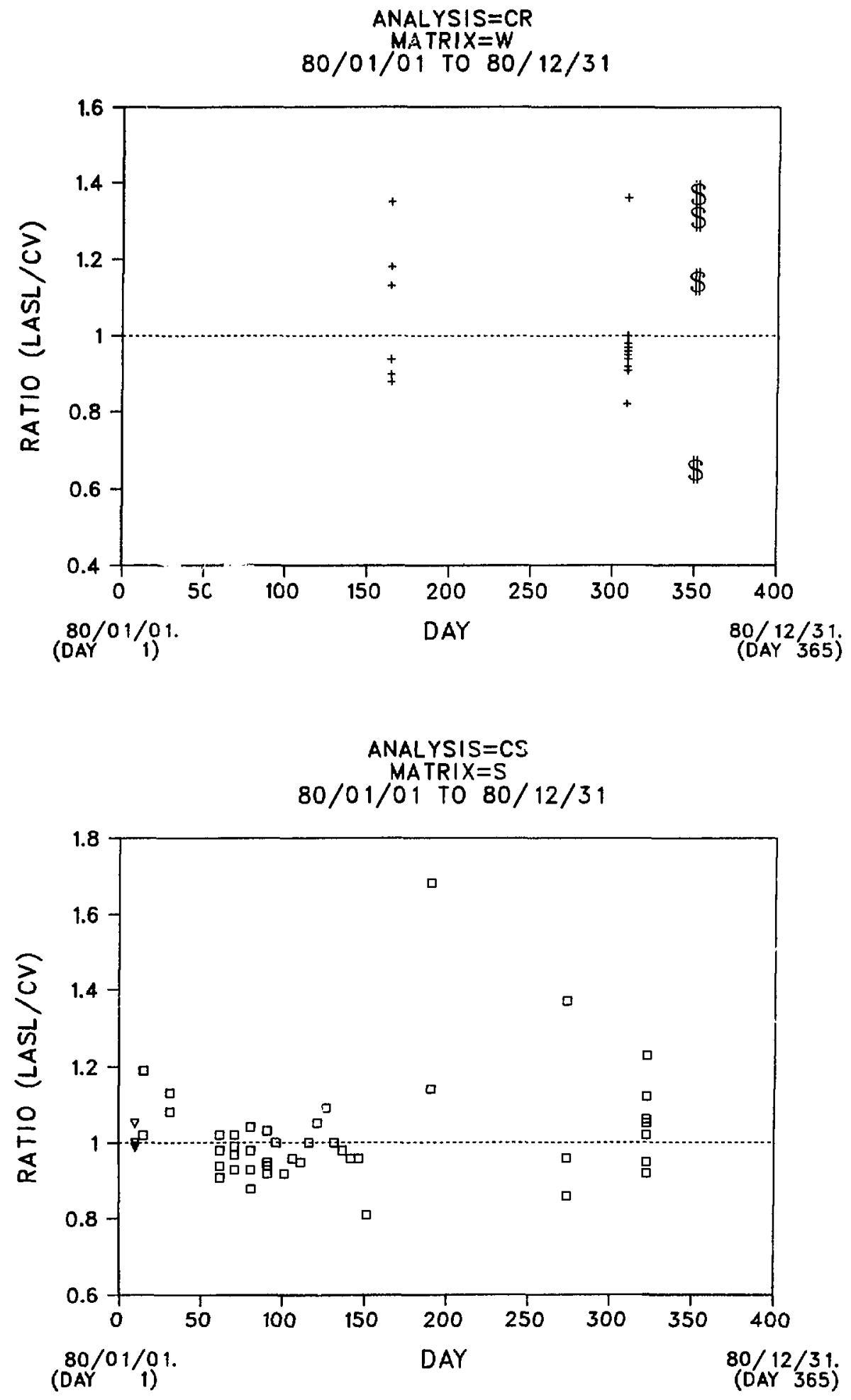

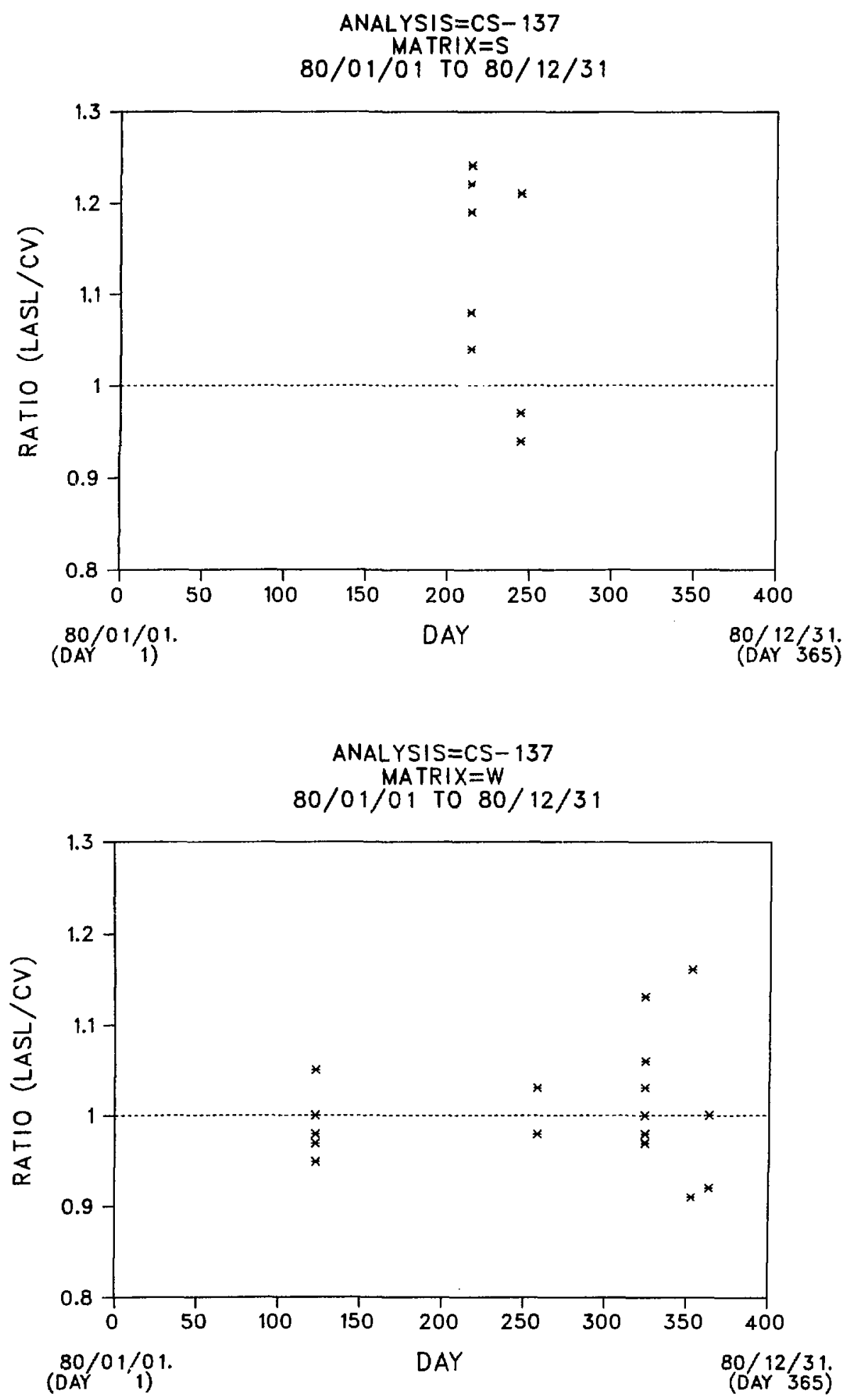

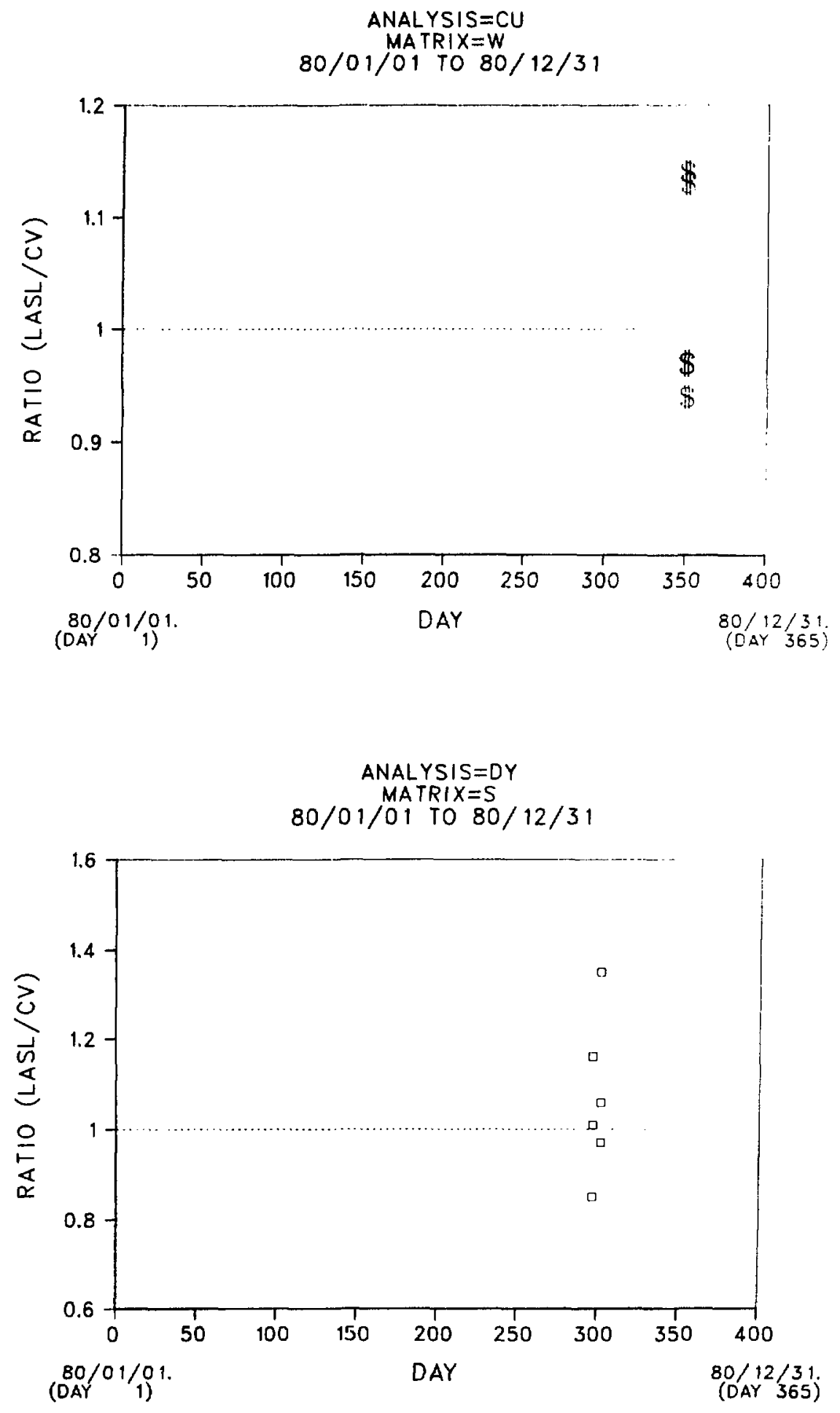

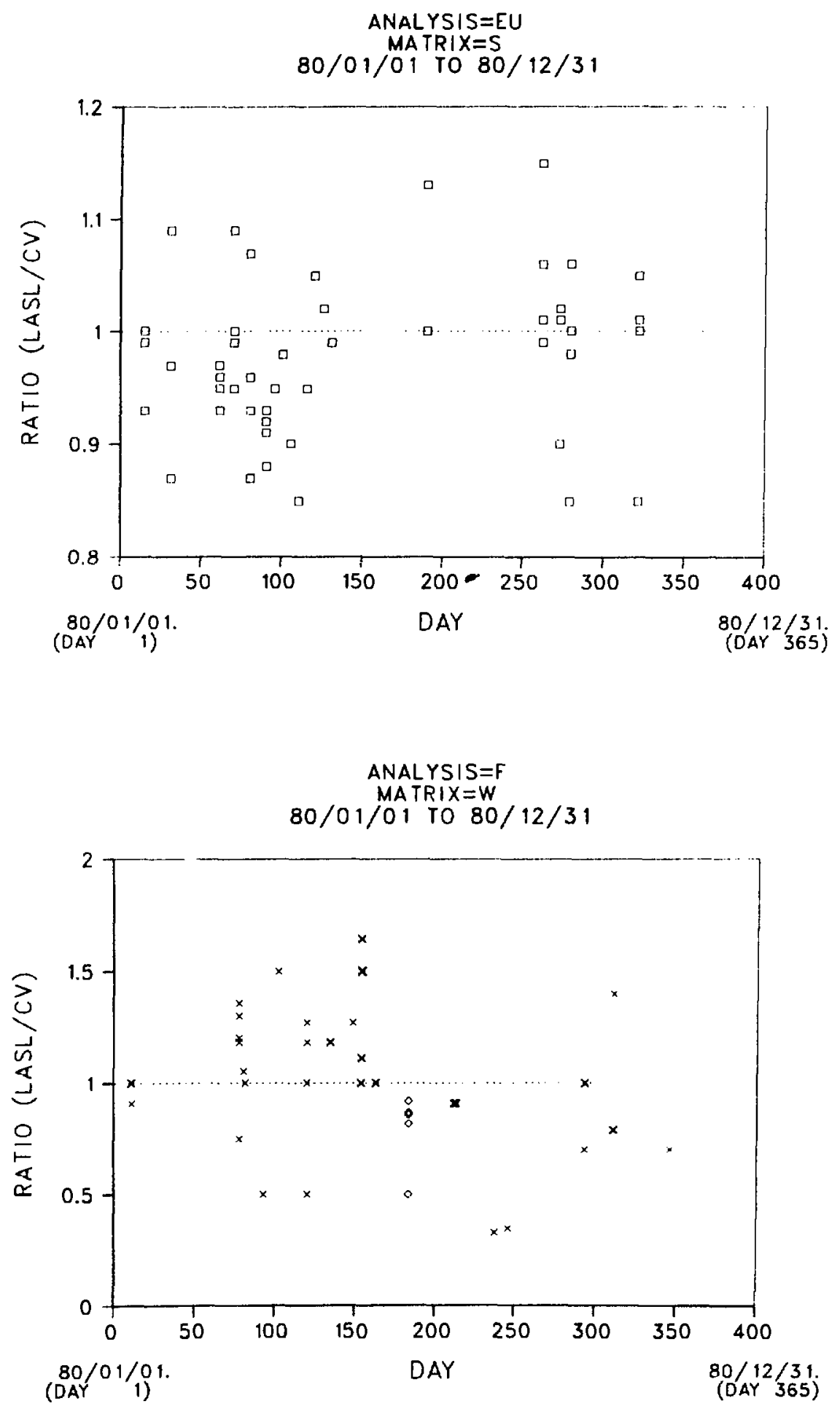

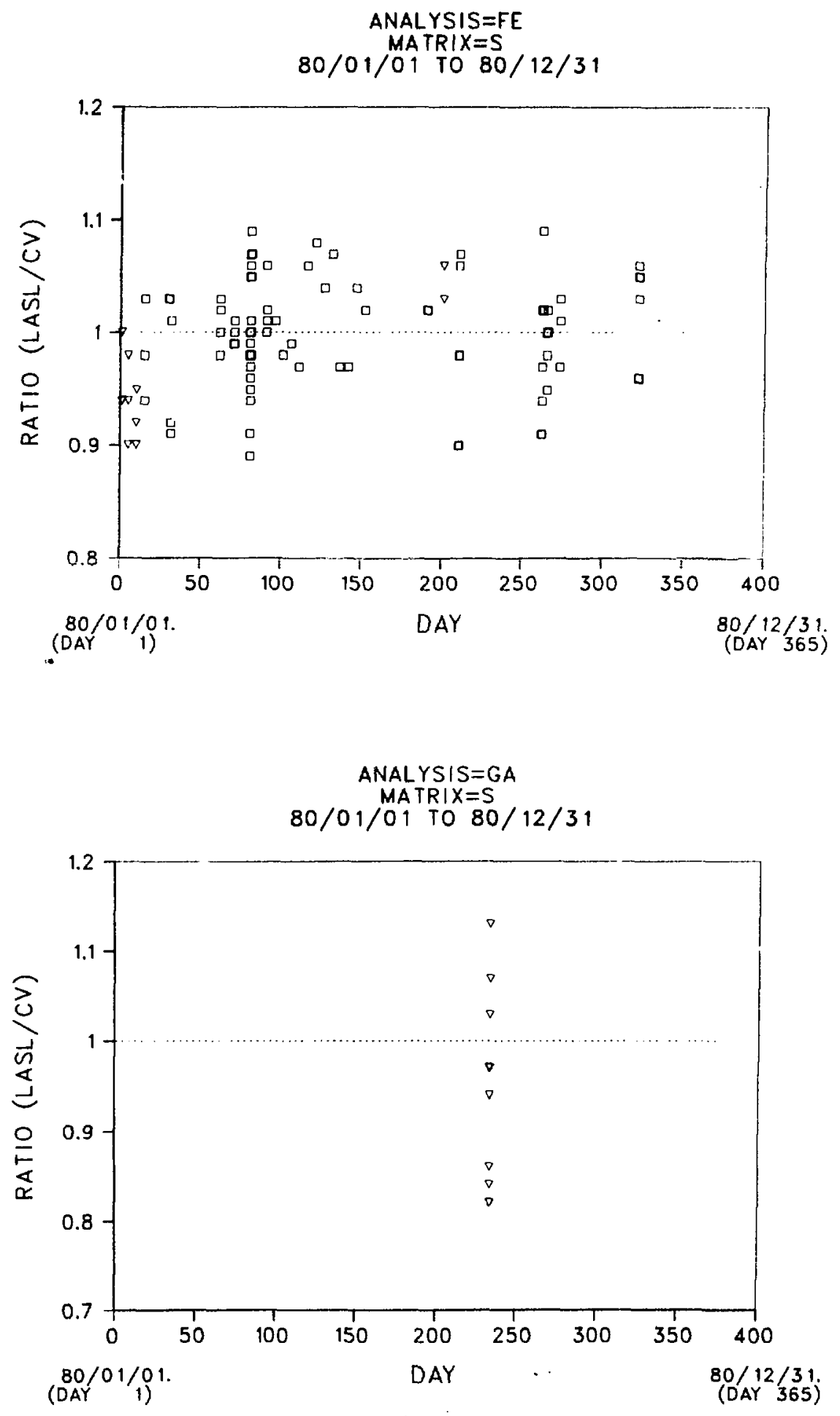

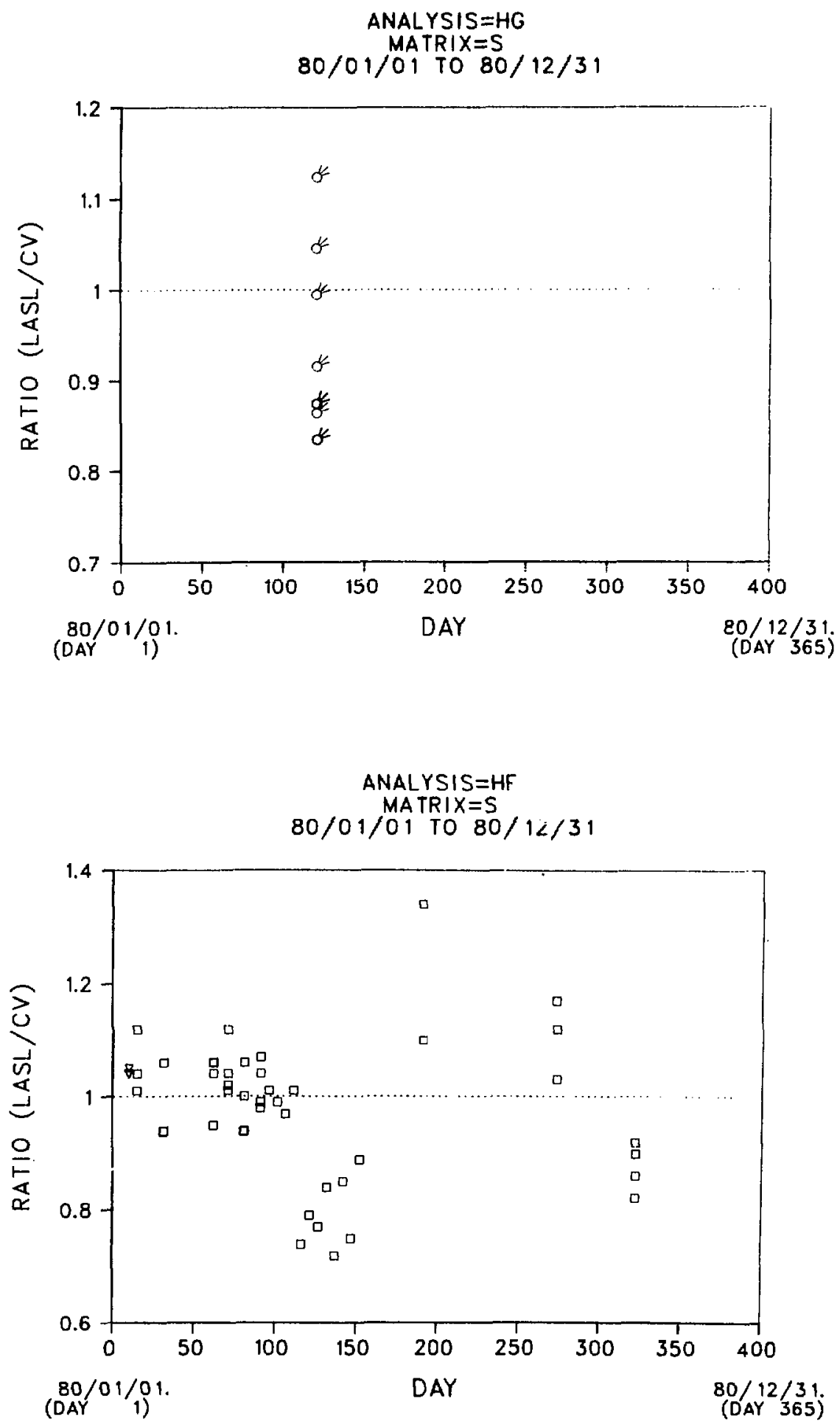

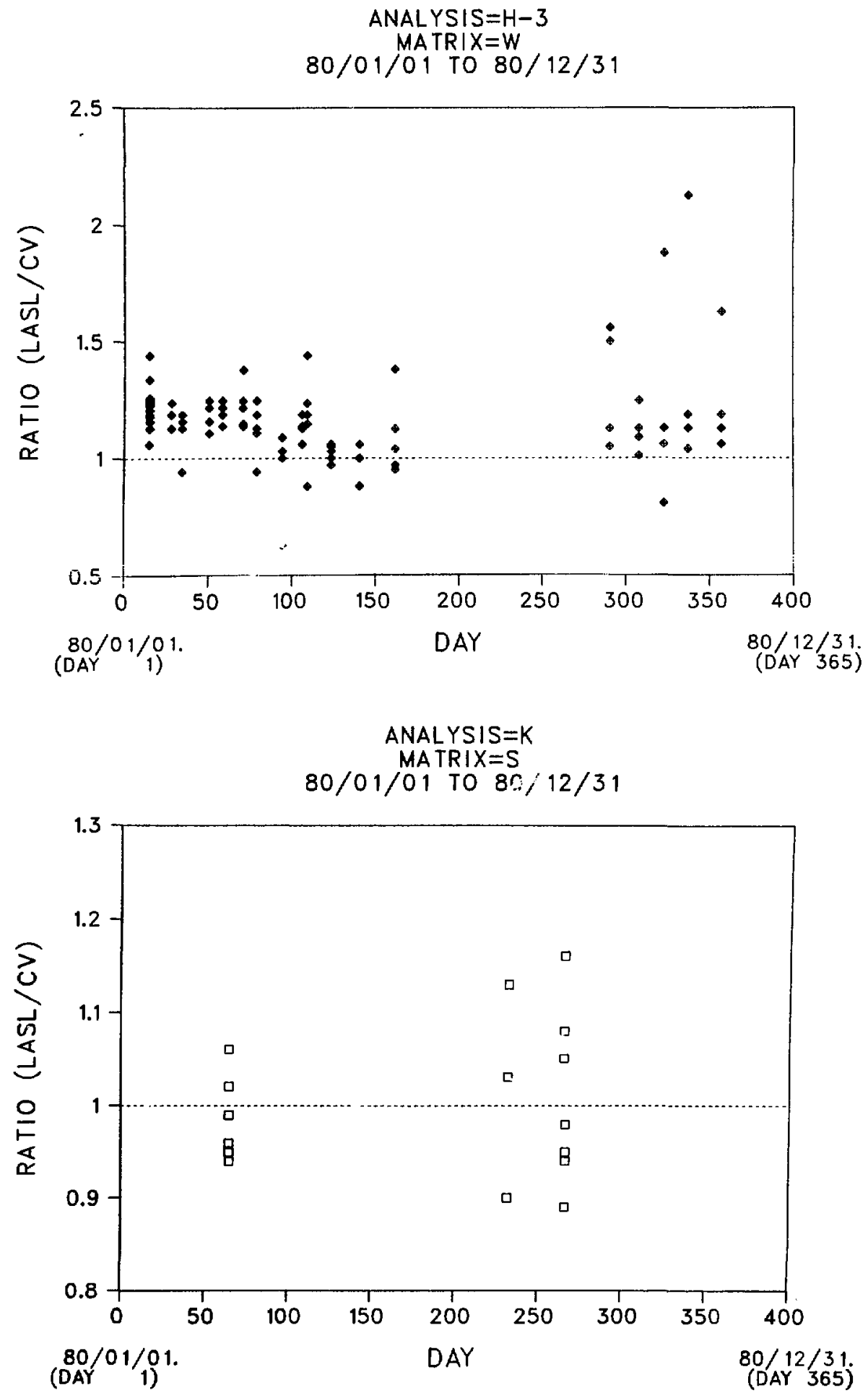

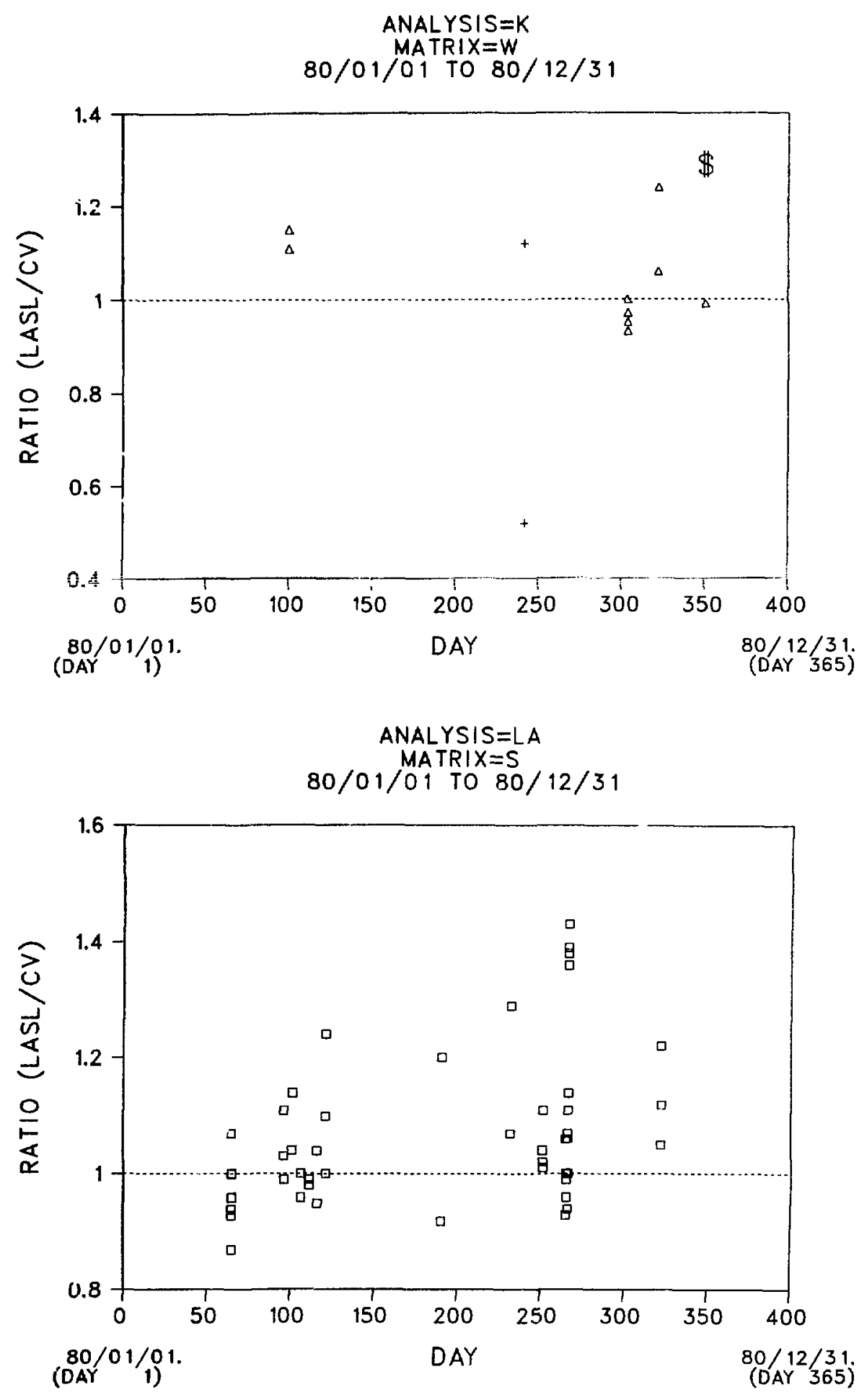

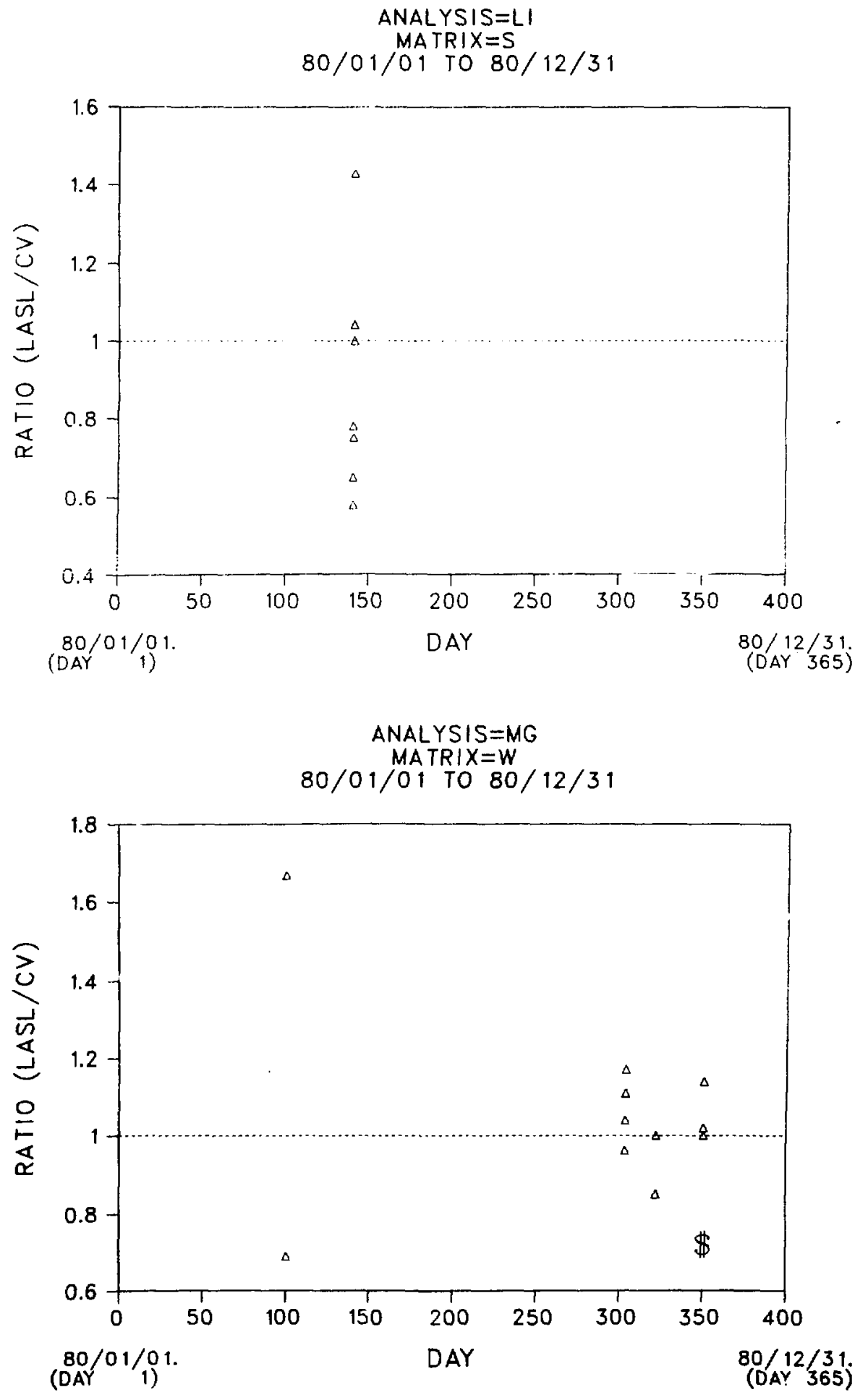

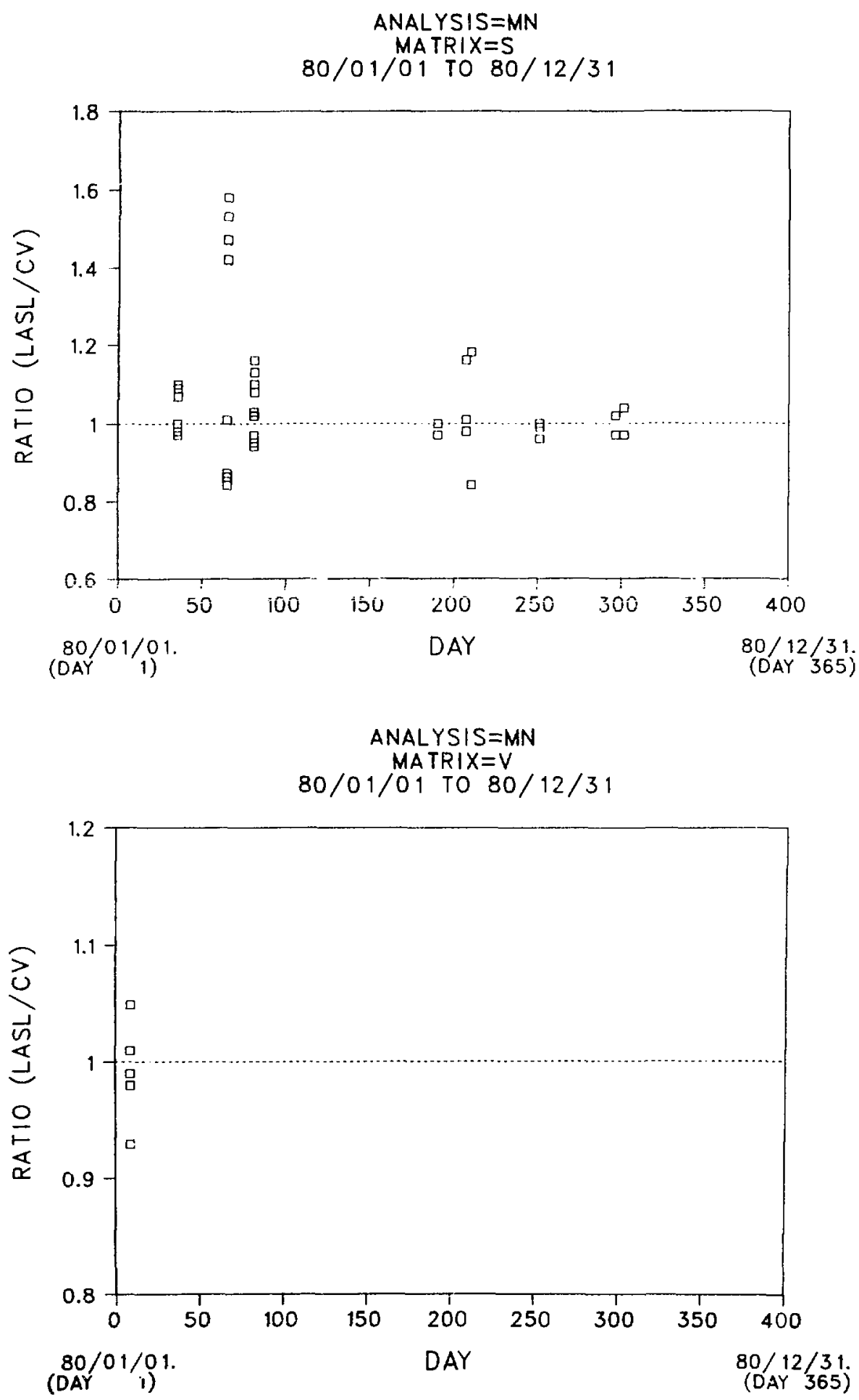

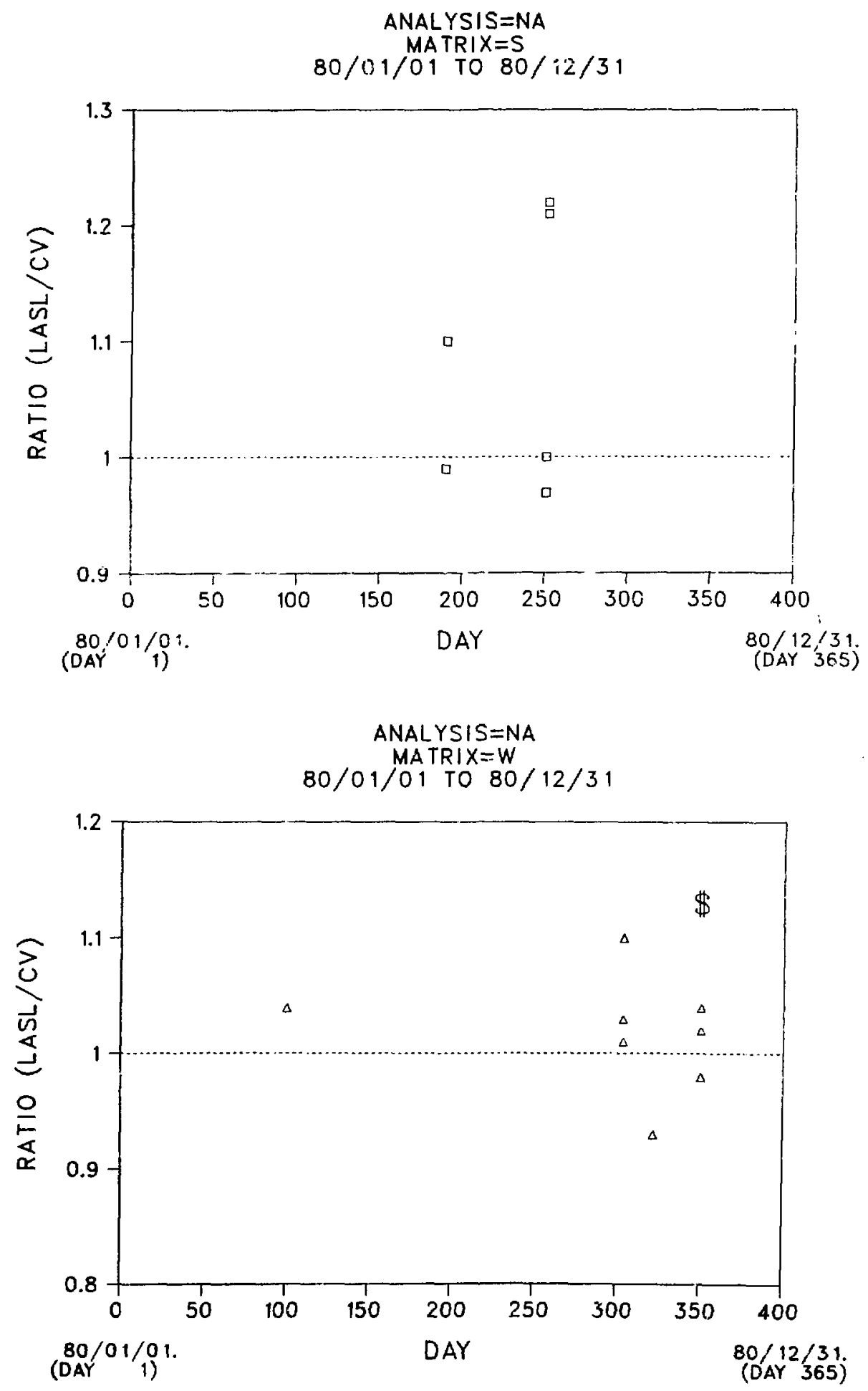

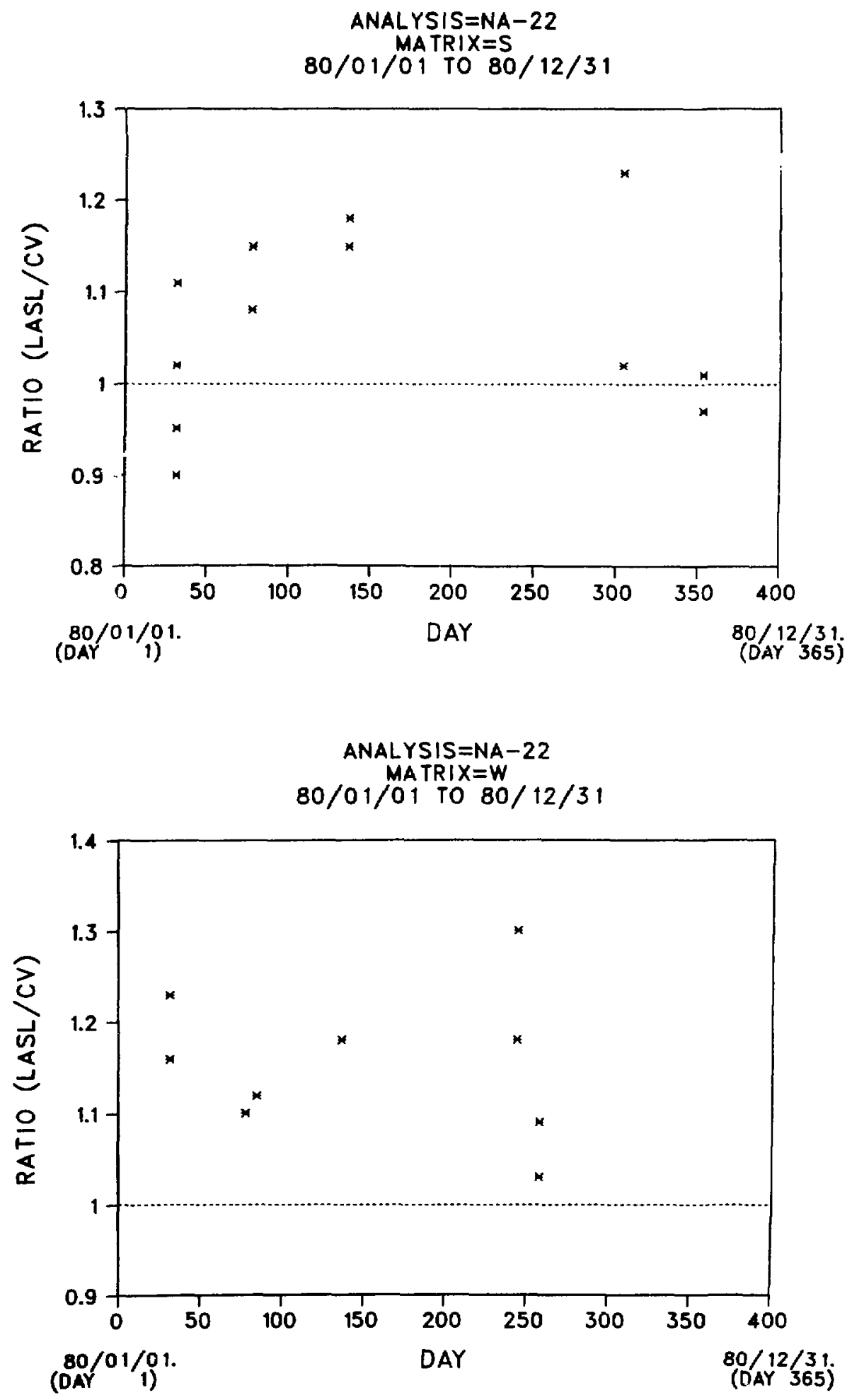

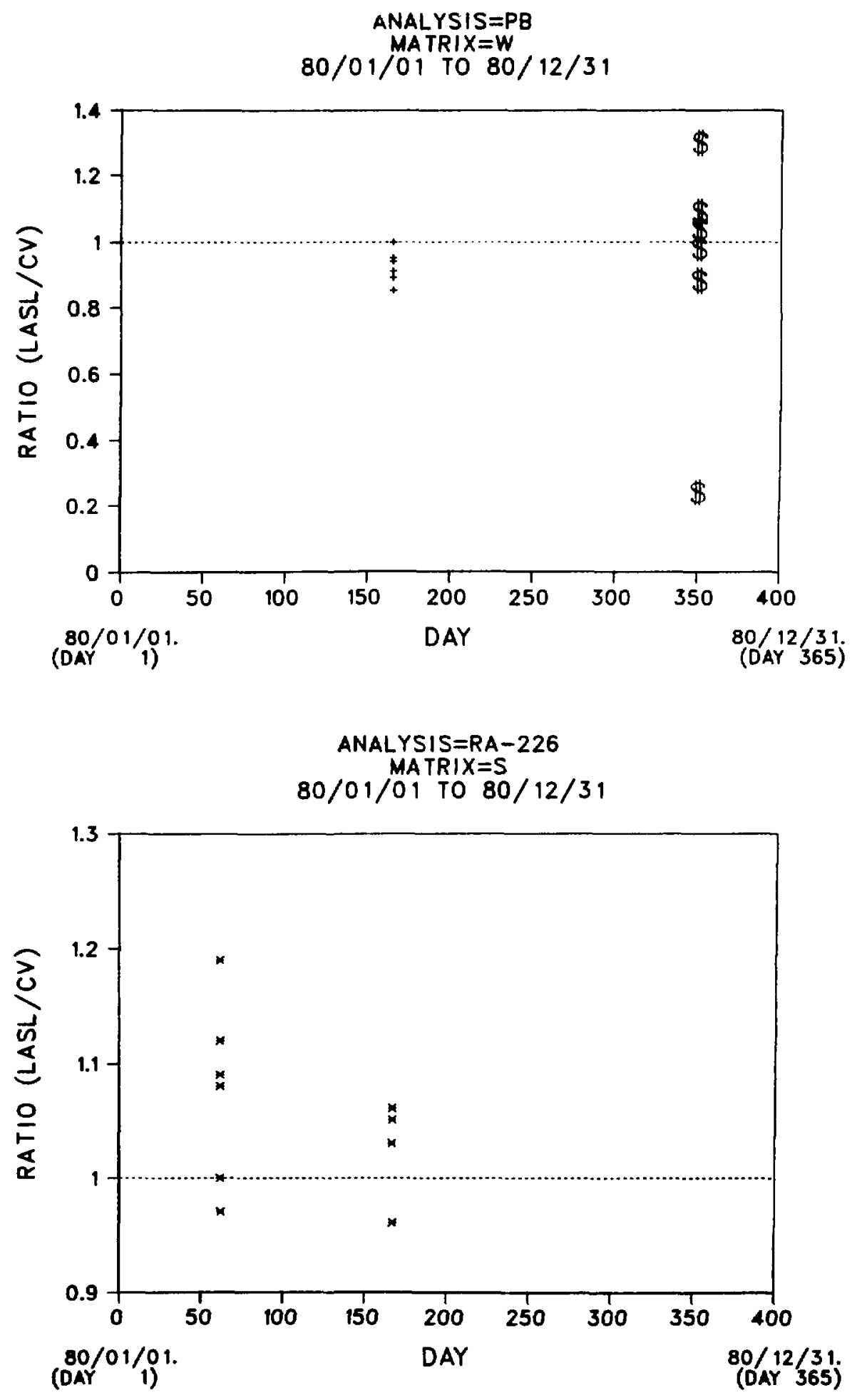

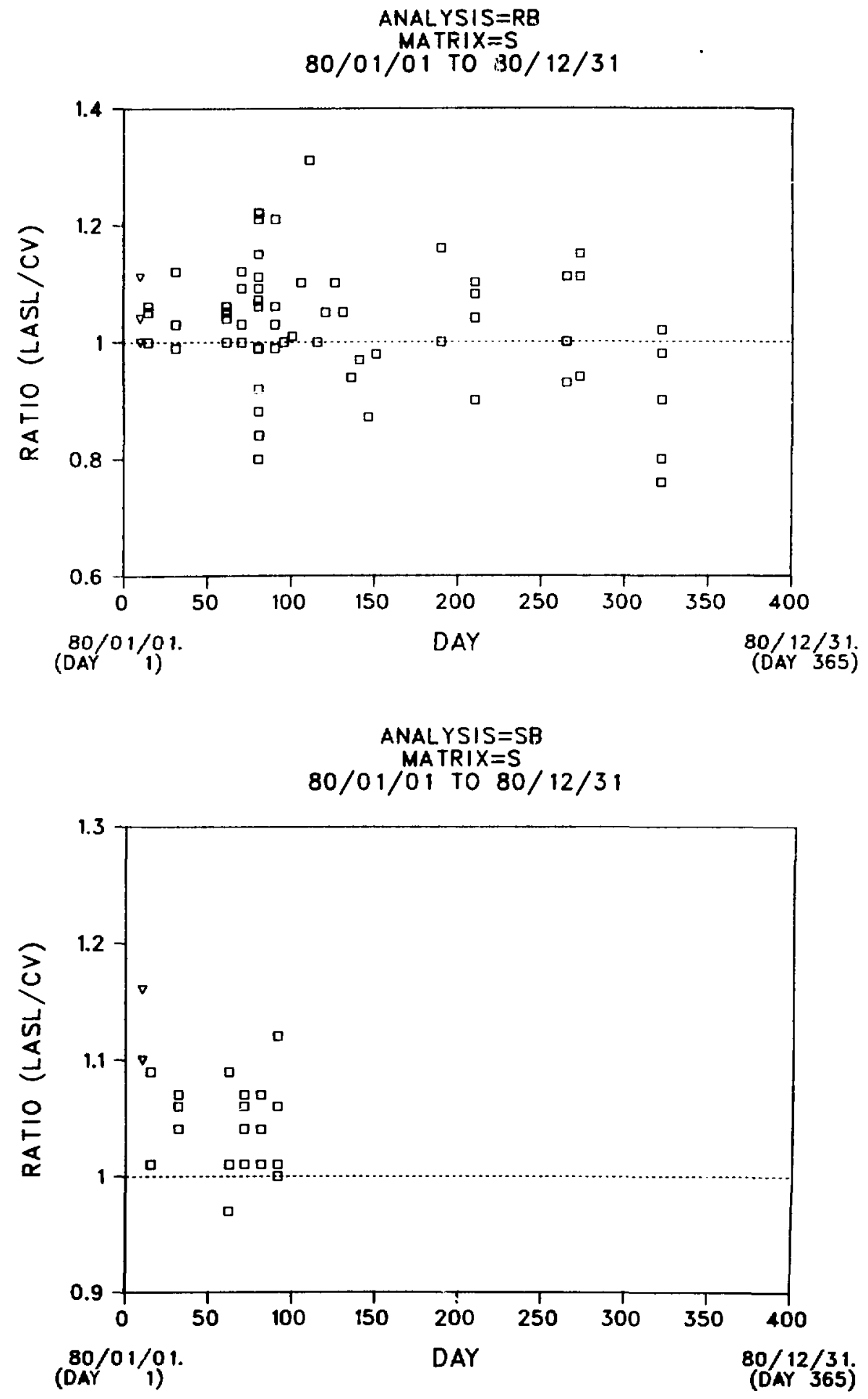

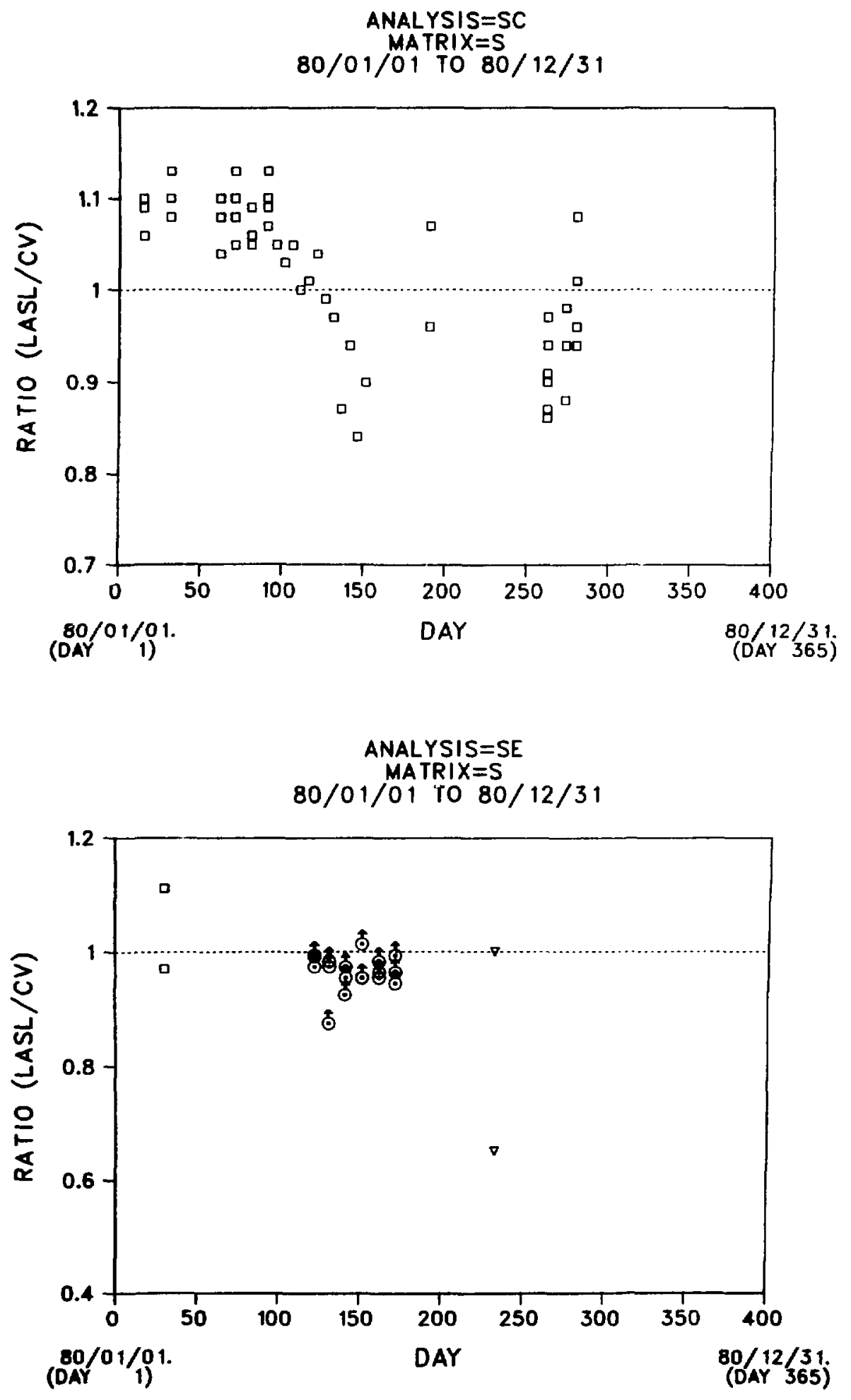

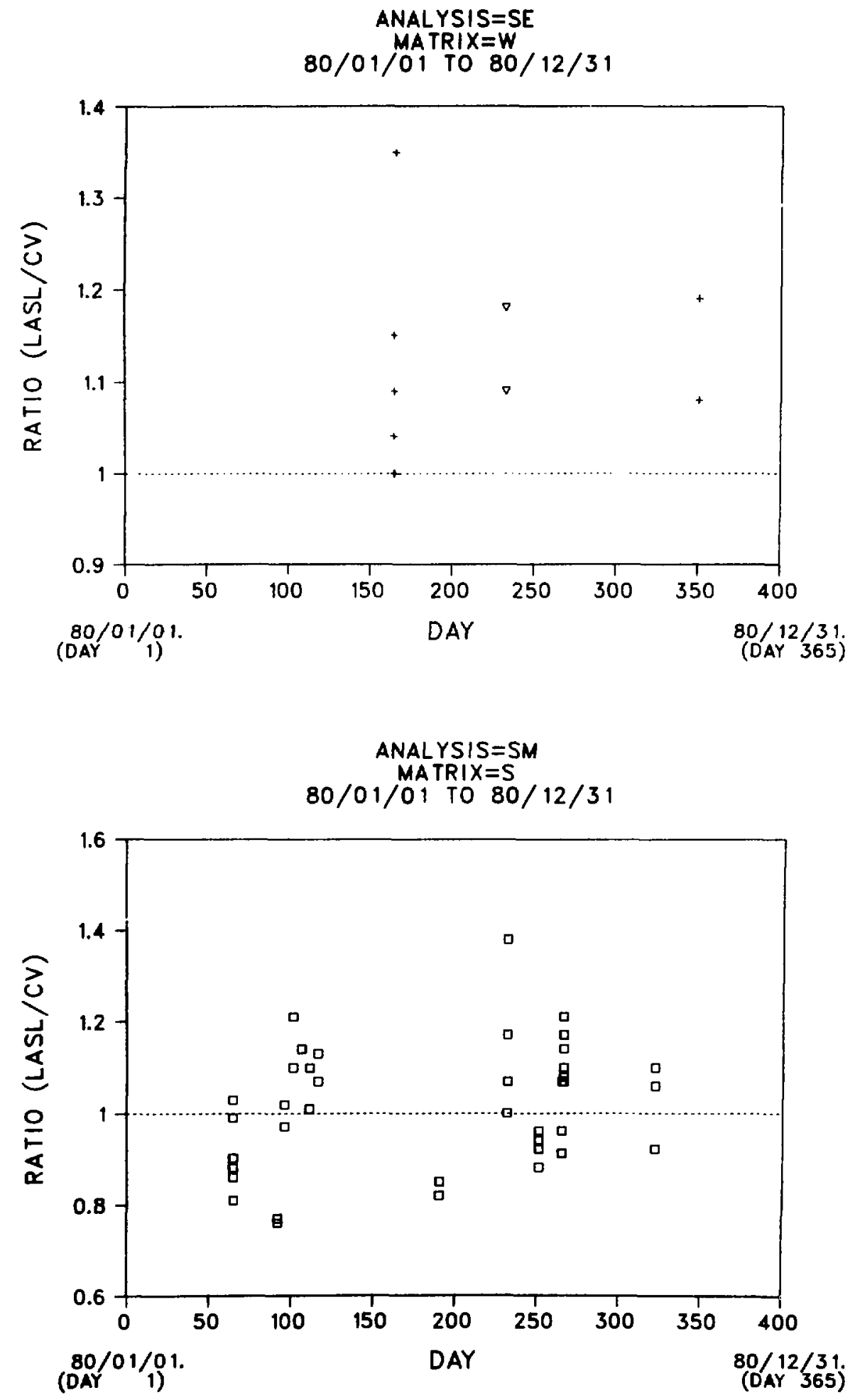

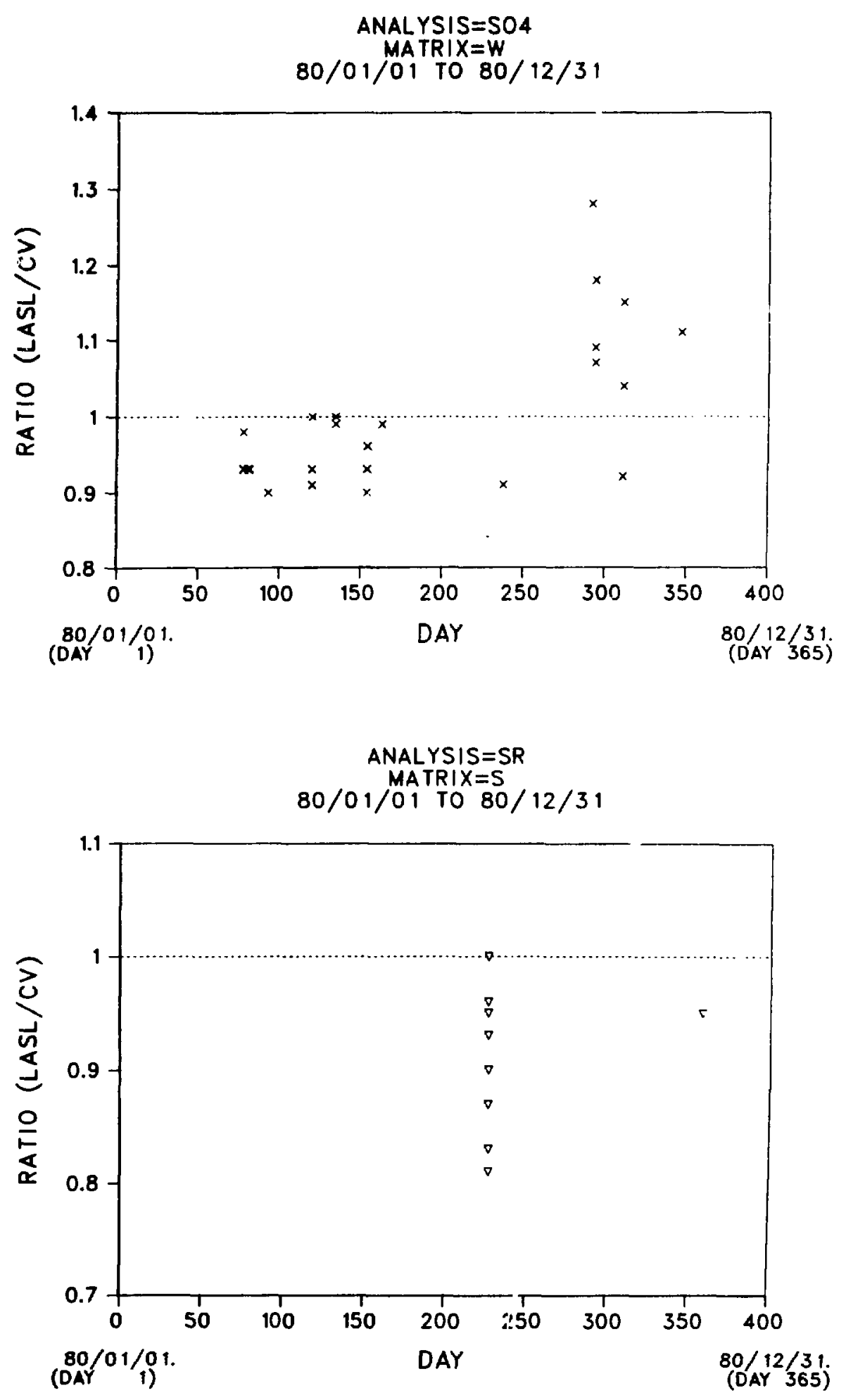

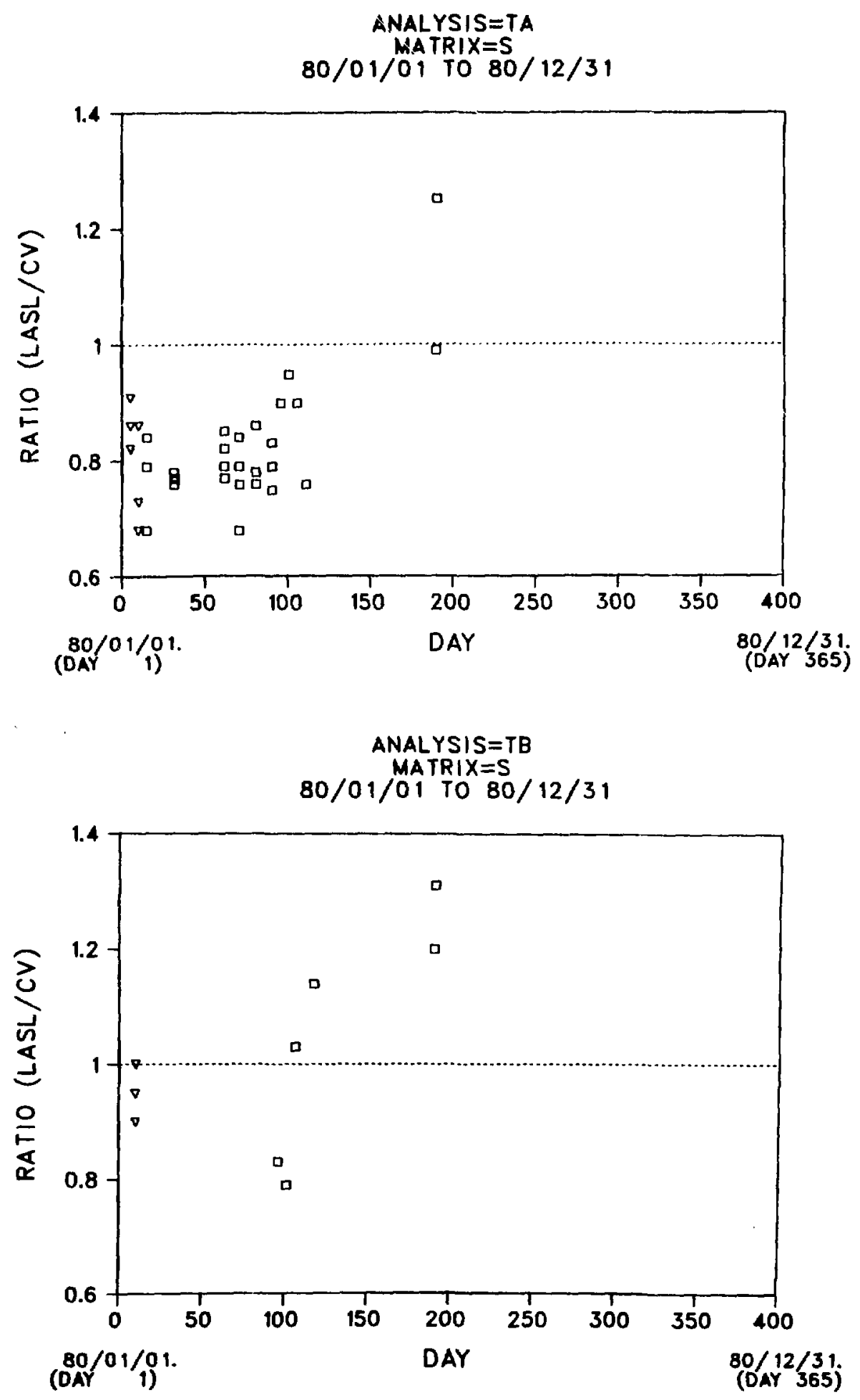

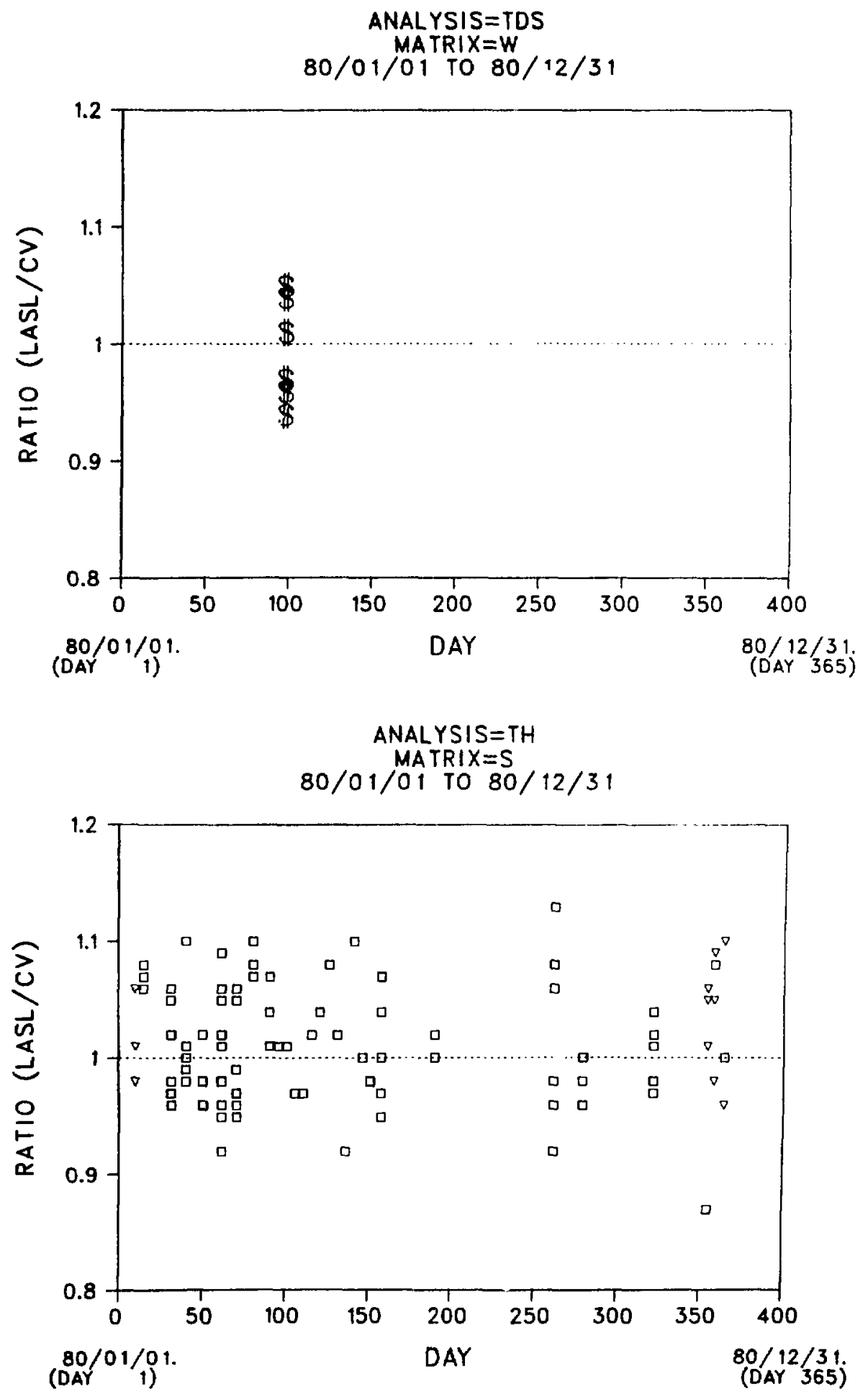

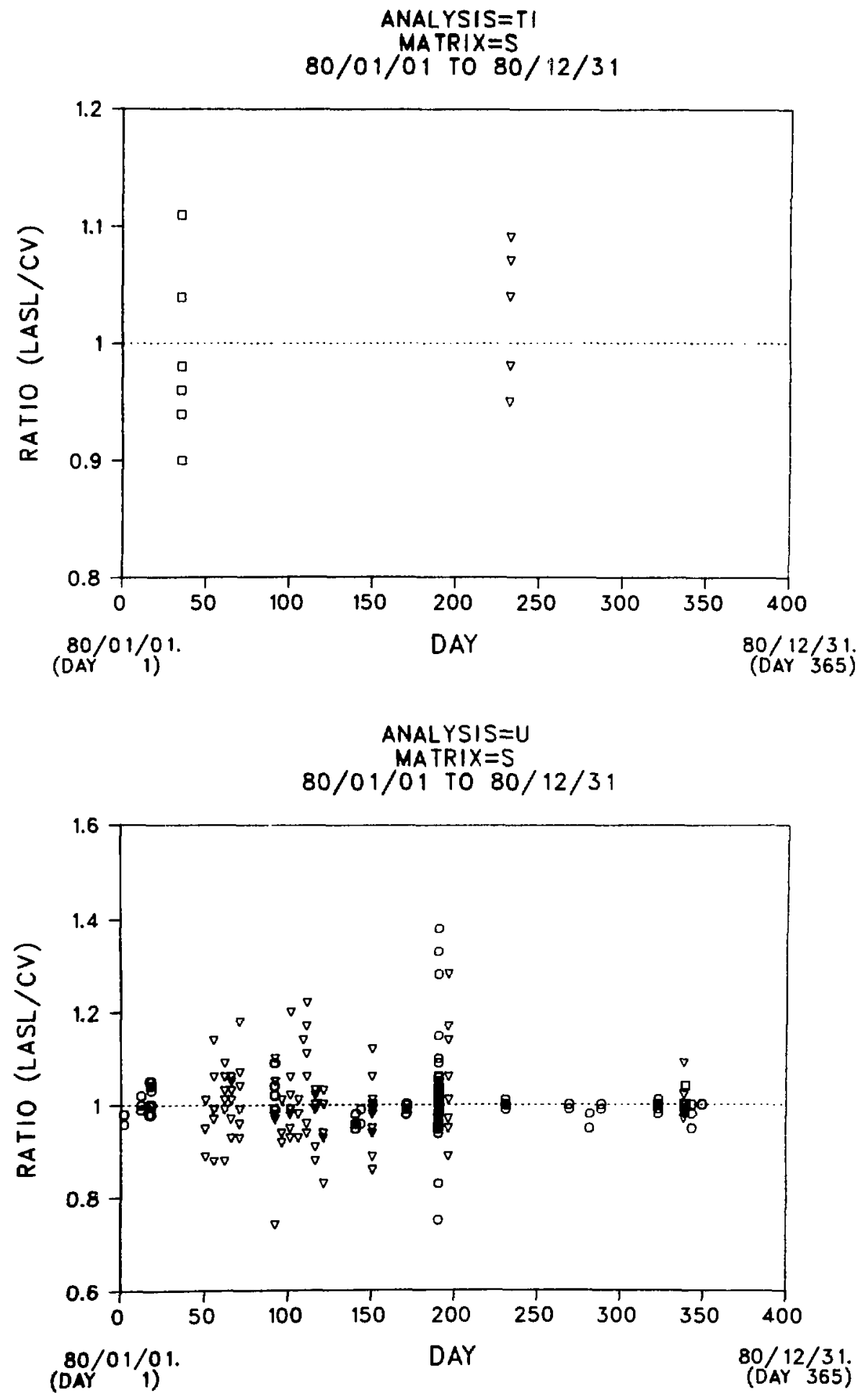

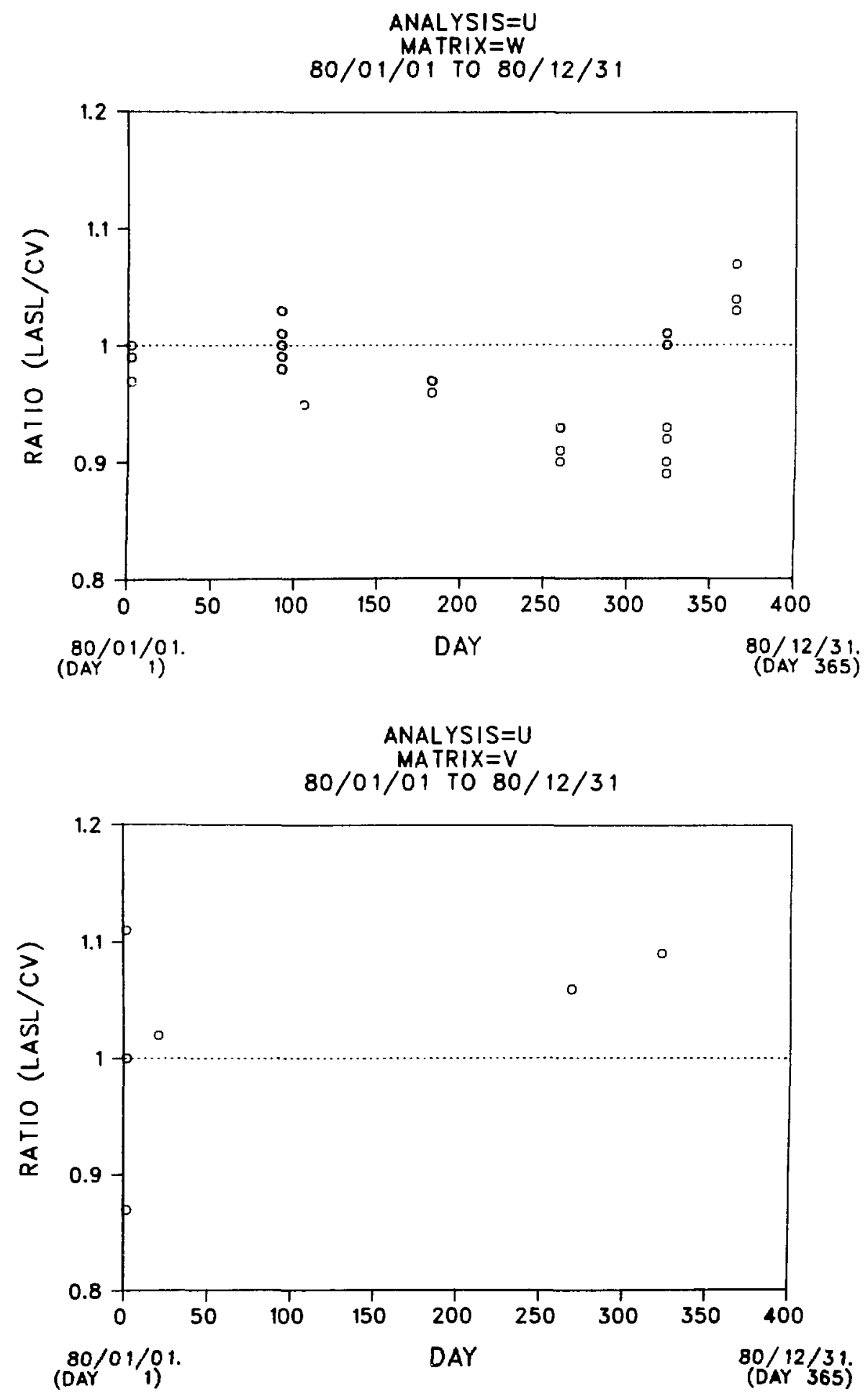

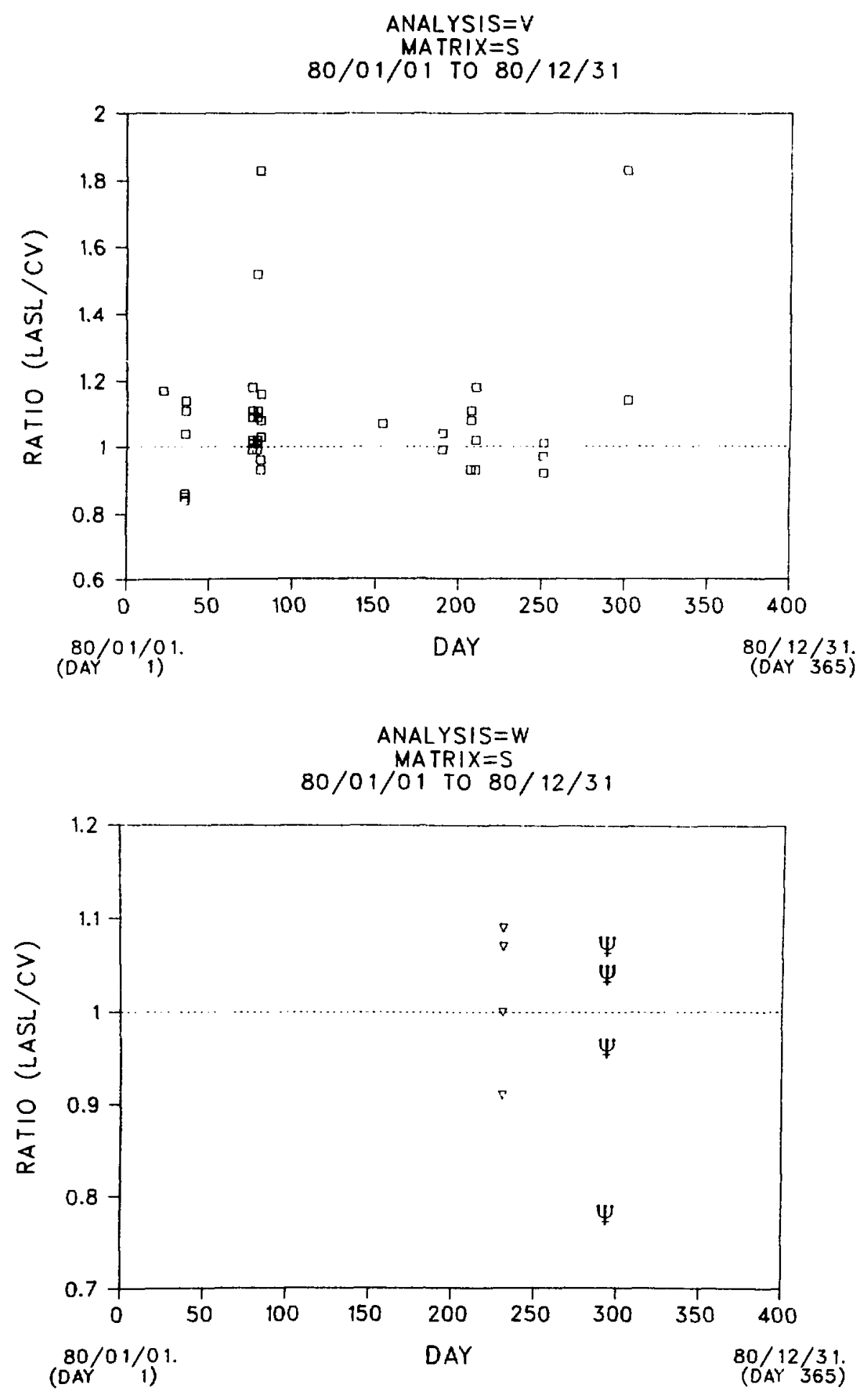

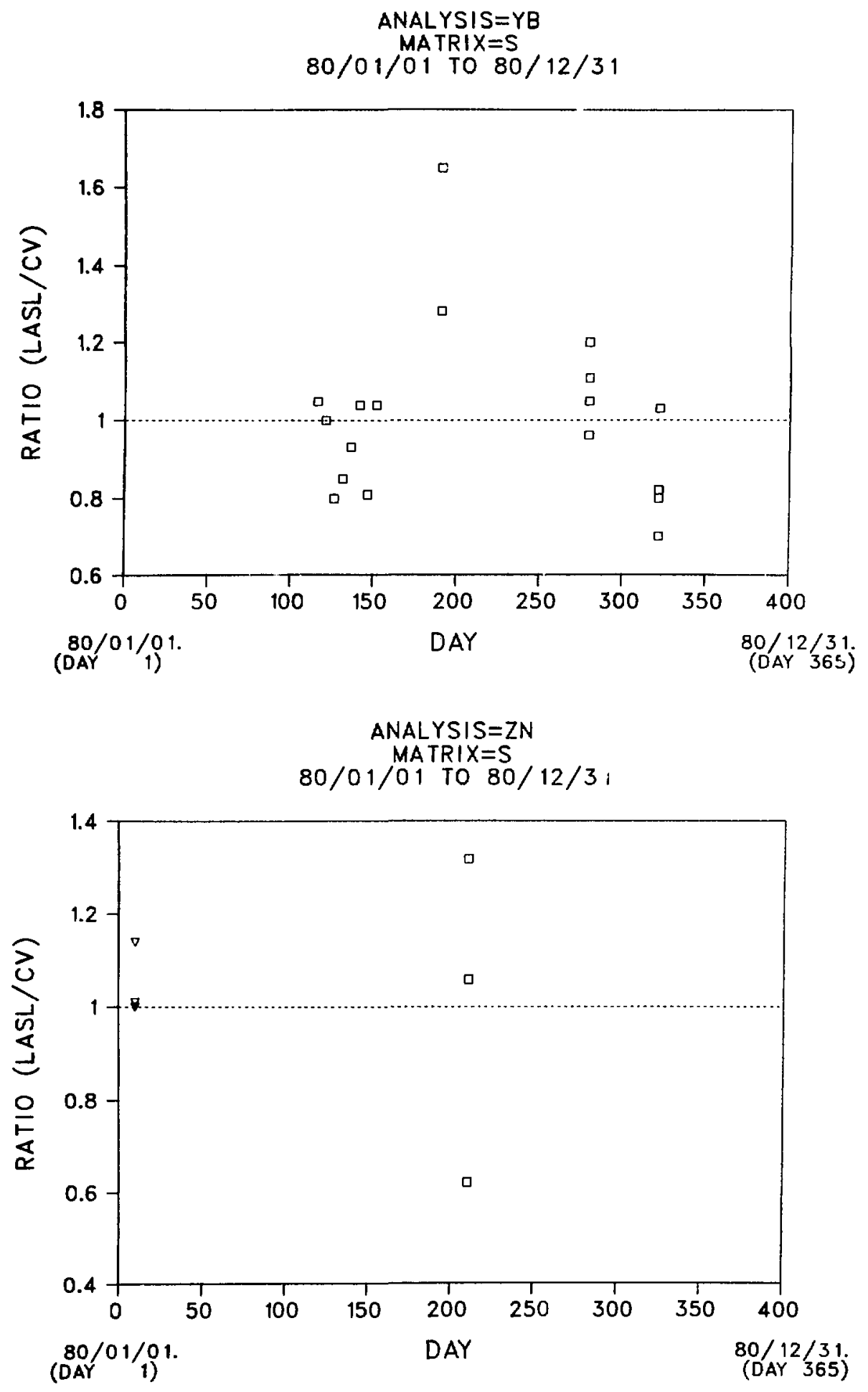


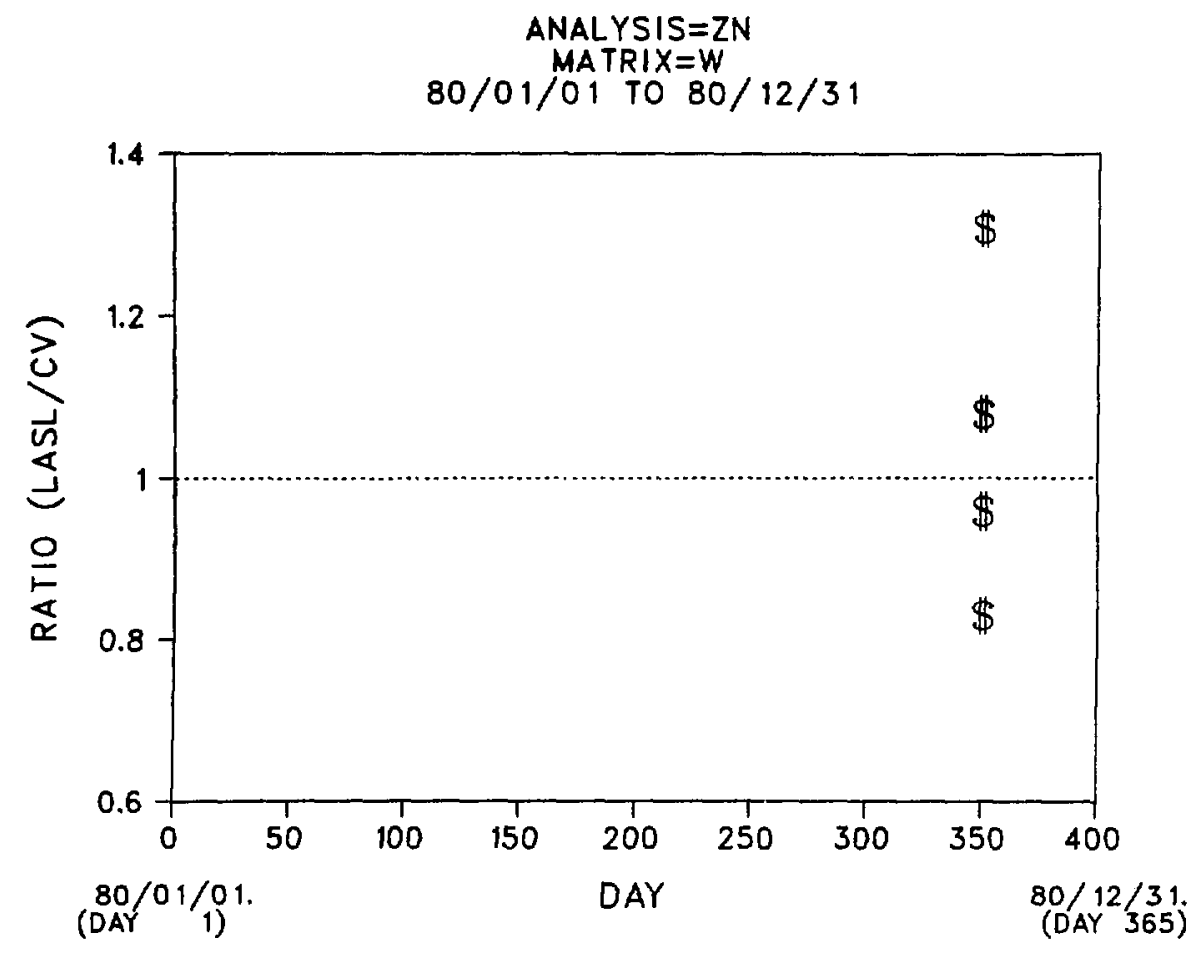


APPENDIX C

QA DATA FOR 1980

107 
SUMMARY OF ELEMENTAL DETERMINATIONS ON NBS, USGS, AND CCRMP SECONDARY REFERENCE MATERIALS

\begin{tabular}{|c|c|c|c|c|c|c|c|}
\hline $\begin{array}{l}\text { H-8 } \\
\text { No. }\end{array}$ & $\begin{array}{l}\text { Agency } \\
\text { ID }\end{array}$ & Element & $\begin{array}{l}\text { Mean Concentration } \\
\pm \text { Standard Deviation } \\
\text { (ppm unless } \\
\text { otherwise indicated) }\end{array}$ & $\begin{array}{c}\text { No. of } \\
\text { Determinations }\end{array}$ & Element & $\begin{array}{c}\text { Mean Concentration } \\
\pm \text { Standard Deviation } \\
\text { (ppm unless } \\
\text { otherwise indicated) }\end{array}$ & $\begin{array}{c}\text { No. of } \\
\text { Determinations }\end{array}$ \\
\hline \multirow[t]{9}{*}{80.00505} & NBS 1643a & $\mathrm{Ag}$ & $2.7 \mathrm{ppb}$ & 2 & $\mathbf{K}$ & 2.3 & 1 \\
\hline & & As & $78 \pm 9 \mathrm{ppb}$ & 26 & $\mathbf{M g}$ & 8.1 & 1 \\
\hline & & $\mathrm{Ba}$ & $52 \pm 6 \mathrm{ppb}$ & 3 & Mo & $110 \mathrm{ppb}$ & 1 \\
\hline & & $\mathrm{Be}$ & $21 \mathrm{ppb}$ & 1 & $\mathrm{Na}$ & 9.3 & 1 \\
\hline & & $\mathrm{Ca}$ & 19.8 & 1 & $\mathrm{Ni}$ & $60 \mathrm{ppb}$ & 2 \\
\hline & & $\mathrm{Cd}$ & $8.0 \pm 1.6 \mathrm{ppb}$ & 28 & $\mathrm{~Pb}$ & $26 \pm 3 \mathrm{ppb}$ & 3 \\
\hline & & Co & $19 \pm 2 \mathrm{ppb}$ & 1 & $\mathrm{Se}$ & $12 \pm 1 \mathrm{ppb}$ & 3 \\
\hline & & $\mathrm{Cr}$ & $18 \pm 5 \mathrm{ppb}$ & 4 & $\mathrm{Zn}$ & $60 \mathrm{ppb}$ & 1 \\
\hline & & $\mathrm{Cu}$ & $17 \mathrm{ppb}$ & 1 & & & \\
\hline 80.00515 & CCRMP SY-2 & B & 86 & 1 & Th & $350 \pm 7$ & 15 \\
\hline 80.00517 & CCRMP MRG-1 & $\mathrm{Cl}$ & 150 & 2 & Sr & 250 & 1 \\
\hline 80.00518 & CCRMP BL-1 & ${ }^{226} \mathrm{Ra}$ & $73 \pm 2 \mathrm{pCi} / \mathrm{g}$ & 3 & $\mathrm{U}$ & $218 \pm 8$ & 35 \\
\hline 80.00519 & CCRMP BL-2 & ${ }^{226} \mathrm{Ra}$ & $1580 \mathrm{pCi} / \mathrm{g}$ & 1 & U & $4490 \pm 56$ & 6 \\
\hline 80.00520 & CCRMP BL-3 & ${ }^{226} \mathrm{Ra}$ & $3420 \mathrm{pCi} / \mathrm{g}$ & 1 & & & \\
\hline 80.00521 & CCRMP BL-4 & ${ }^{226} \mathrm{Ra}$ & $608 \pm 13 \mathrm{pCi} / \mathrm{g}$ & 3 & & & \\
\hline \multirow[t]{2}{*}{80.00522} & CCRMP DH-1 & ${ }^{226} \mathrm{Ra}$ & $670 \mathrm{pCi} / \mathrm{g}$ & 2 & $\mathrm{U}$ & $1763 \pm 106$ & 15 \\
\hline & & Th & $1125 \pm 25$ & 3 & & & \\
\hline
\end{tabular}


TABLE C-I (cont)

\begin{tabular}{|c|c|c|c|c|c|c|c|}
\hline $\begin{array}{l}\text { H-8 } \\
\text { No. }\end{array}$ & $\begin{array}{c}\text { Agency } \\
\text { ID }\end{array}$ & Element & $\begin{array}{l}\text { Mean Concentration } \\
\pm \text { Standard Deviation } \\
\text { (ppm unless } \\
\text { otherwise indicated) }\end{array}$ & $\begin{array}{c}\text { No. of } \\
\text { Determinations } \\
\end{array}$ & Element & $\begin{array}{c}\text { Mean Concentration } \\
\pm \text { Standard Deviation } \\
\text { (ppm unless } \\
\text { otherwise indicated) }\end{array}$ & $\begin{array}{c}\text { No. of } \\
\text { Determinations } \\
\end{array}$ \\
\hline 80.00522 & CCRMP DĹ-1 & Th & $82 \pm 6$ & 6 & $\mathbf{U}$ & $40.3 \pm 2.1$ & 107 \\
\hline \multirow[t]{2}{*}{80.00531} & NBS 1570 & $\mathrm{Ca}$ & $1.21 \%$ & 2 & $\mathbf{M n}$ & 160 & 2 \\
\hline & & $\mathrm{Cl}$ & 6000 & 2 & $\mathrm{Na}$ & $1.48 \%$ & 2 \\
\hline \multirow[t]{4}{*}{80.00532} & NBS 1571 & $\mathrm{Ca}$ & $1.58 \%$ & 1 & $\mathbf{M n}$ & $92 \pm 4$ & 3 \\
\hline & & $\mathrm{Cl}$ & $700 \pm 60$ & 5 & $\mathrm{Na}$ & 92 & 1 \\
\hline & t & $\mathrm{Hg}$ & $165 \pm 5 \mathrm{ppb}$ & 3 & $\mathbf{U}$ & $30 \mathrm{ppb}$ & 2 \\
\hline & & $\mathbf{K}$ & $1.4 \pm 0.2 \%$ & 3 & & & \\
\hline 80.00533 & NBS 1573 & B & $32 \pm 3$ & 7 & & & \\
\hline 80.00534 & NBS 1575 & B & $17 \pm 1$ & 6 & & & \\
\hline \multirow[t]{2}{*}{80.00535} & NBS 1577 & $\mathrm{Cl}$ & 2460 & 1 & $\mathrm{Mn}$ & 10 & 1 \\
\hline & & $\mathbf{K}$ & $1.06 \%$ & 1 & $\mathrm{Na}$ & 2550 & 1 \\
\hline \multirow[t]{13}{*}{80.00537} & NBS 1633 & As & $59 \pm 2$ & 3 & $\mathrm{Nd}$ & 60 & 2 \\
\hline & & $\mathbf{B a}$ & $2840 \pm 180$ & 28 & $\mathrm{Ni}$ & $84 \pm 2$ & 3 \\
\hline & & $\mathrm{Br}$ & $6.9 \pm 0.3$ & 3 & $\mathbf{R} \mathbf{b}$ & $118 \pm 7$ & 25 \\
\hline & & $\mathrm{Ce}$ & $149 \pm 7$ & 25 & $\mathbf{S b}$ & $7.2 \pm 0.3$ & 25 \\
\hline & & $\mathrm{Co}$ & $40 \pm 2$ & 28 & $\mathrm{Sc}$ & $28.3 \pm 0.7$ & 22 \\
\hline & & $\mathrm{Cr}$ & $128 \pm 5$ & 20 & $\mathrm{Se}$ & $9.1 \pm 0.3$ & 18 \\
\hline & & Cs & $8.7 \pm 0.7$ & 26 & $\mathrm{Sr}$ & 1300 & 2 \\
\hline & & Dy & 10.2 & 1 & $\mathrm{Ta}$ & $1.74 \pm 0.12$ & 28 \\
\hline & & $\mathrm{Eu}$ & $2.50 \pm 0.16$ & 22 & $\mathrm{~Tb}$ & $2.0 \pm 0.1$ & 3 \\
\hline & & $\mathrm{Fe}$ & $6.1 \pm 0.3 \%$ & 33 & Th & $25.0 \pm 0.9$ & 25 \\
\hline & & $\mathrm{Ga}$ & $41 \pm 1$ & 3 & w & $5.8 \pm 0.3$ & 3 \\
\hline & & $\mathrm{Hf}$ & $8.0 \pm 0.4$ & 25 & $\mathrm{Zn}$ & $221 \pm 16$ & 3 \\
\hline & & $\mathbf{K}$ & $1.97 \%$ & 1 & $\mathrm{Zr}$ & $286 \pm 8$ & 3 \\
\hline
\end{tabular}




\begin{tabular}{|c|c|c|c|c|c|c|c|}
\hline $\begin{array}{l}\text { H-8 } \\
\text { No. }\end{array}$ & $\begin{array}{l}\text { Agency } \\
\text { ID }\end{array}$ & Element & $\begin{array}{l}\text { Mean Concentration } \\
\pm \text { Standard Deviation } \\
\text { (ppm unleas } \\
\text { otherwice indicated) }\end{array}$ & $\begin{array}{c}\text { No. of } \\
\text { Determinations }\end{array}$ & Element & $\begin{array}{l}\text { Mean Concentration } \\
\pm \text { Standard Deviation } \\
\text { (ppm unless } \\
\text { otherwise indicated) }\end{array}$ & $\begin{array}{c}\text { No. of } \\
\text { Determinations }\end{array}$ \\
\hline 80.00540 & NBS $1642 A$ & $\mathbf{H g}$ & $1.1 \pm 0.1 \mathrm{ppb}$ & 4 & & & \\
\hline \multirow[t]{13}{*}{80.00570} & USGS AGV-1 & $\mathbf{A} 1$ & $8.94 \%$ & 2 & Mn & 748 & 2 \\
\hline & & As & 1.2 & 1 & $\mathrm{Na}$ & $3.12 \pm 0.04 \%$ & 3 \\
\hline & & $\mathrm{Ba}$ & 1295 & 2 & $\mathbf{R b}$ & 72 & 2 \\
\hline & & Ce & 67 & 1 & Sc & 12.3 & 2 \\
\hline & & Co & 15.3 & 2 & Sm & $5.2 \pm 0.2$ & 3 \\
\hline & & $\mathrm{Cr}$ & 12 & 2 & $\mathrm{Sr}$ & 660 & 1 \\
\hline & & Cs & 1.4 & 2 & $\mathrm{Ta}$ & 0.89 & 1 \\
\hline & & $\mathbf{E u}$ & 1.7 & 2 & $\mathrm{~Tb}$ & 0.92 & 1 \\
\hline & & $\mathrm{Fe}$ & $4.80 \%$ & 2 & Th & 6.4 & 1 \\
\hline & & $\mathrm{Ga}$ & 19.3 & 1 & $\mathrm{Ti}$ & 6040 & 1 \\
\hline & & Hf & 5.8 & 2 & $\mathbf{U}$ & $1.95 \pm 0.06$ & 4 \\
\hline & & $\mathbf{L a}$ & $40 \pm 2$ & 3 & $\mathbf{v}$ & 122 & 2 \\
\hline & & Lu & 0.41 & 1 & $\mathbf{Y b}$ & 2.8 & 1 \\
\hline 80.00571 & USGS G-2 & $\mathbf{U}$ & $2.2 \pm 0.1$ & 3 & & & \\
\hline \multirow[t]{11}{*}{80.00572} & USGS BCR-1 & Al & $7.19 \%$ & 2 & $\mathrm{Mn}$ & 1400 & 2 \\
\hline & & As & 0.5 & 1 & $\mathrm{Na}$ & $2.45 \pm 0.14 \%$ & 3 \\
\hline & & $\mathrm{Ba}$ & 850 & 2 & $\mathbf{R b}$ & 49 & 2 \\
\hline & & Ce & 45 & 1 & Sc & 34 & 2 \\
\hline & & Co & 36 & 2 & Sm & $5.9 \pm 0.4$ & 3 \\
\hline & & $\mathrm{Cr}$ & 17 & 2 & $\mathbf{S r}$ & 330 & 1 \\
\hline & & Cs & 1.4 & 2 & $\mathrm{Ta}$ & 1.14 & 1 \\
\hline & & $\mathbf{E u}$ & 2.1 & 2 & $\mathbf{T b}$ & 1.2 & 1 \\
\hline & & $\mathrm{Fe}$ & $9.61 \%$ & 2 & Th & 6.1 & 1 \\
\hline & & $\mathrm{Ga}$ & 20.6 & 1 & $\mathrm{Ti}$ & $1.27 \%$ & 1 \\
\hline & & Hf & 5.9 & 2 & $\mathbf{U}$ & $1.8 \pm 0.1$ & 3 \\
\hline
\end{tabular}


TABLE C-I (cont)

\begin{tabular}{|c|c|c|c|c|c|c|c|}
\hline $\begin{array}{l}\text { H-8 } \\
\text { No. }\end{array}$ & $\begin{array}{c}\text { Agency } \\
\text { ID }\end{array}$ & Element & $\begin{array}{l}\text { Mean Concentration } \\
\pm \text { Standard Devintion } \\
\text { (ppom unless } \\
\text { otherwice indicated) }\end{array}$ & $\begin{array}{c}\text { No. of } \\
\text { Determinations }\end{array}$ & Element & $\begin{array}{l}\text { Mean Concentration } \\
\pm \text { Standard Deviation } \\
\text { (ppm unlese } \\
\text { otherwice indicated) }\end{array}$ & $\begin{array}{c}\text { No. of } \\
\text { Determinutions }\end{array}$ \\
\hline & & La & $26 \pm 2$ & 3 & $\mathbf{v}$ & 400 & 2 \\
\hline & & $\mathbf{L u}$ & 1 & 1 & $\mathbf{Y b}$ & 4.3 & 1 \\
\hline 80.00574 & USGS GSP-1 & $\mathbf{U}$ & $2.6 \pm 0.1$ & 3 & & & \\
\hline \multirow[t]{7}{*}{80.00575} & USGS GXR-1 & Al & $3.4 \%$ & 1 & $\mathbf{L a}$ & $8.5 \pm 0.2$ & 4 \\
\hline & & As & $430 \pm 60$ & 5 & $\mathrm{Li}$ & 10 & 2 \\
\hline & & Cd & 3.8 & 2 & $\mathrm{Na}$ & $610 \pm 10$ & 4 \\
\hline & & Co & $10.0 \pm 0.8$ & 3 & $\mathrm{Se}$ & 12 & 1 \\
\hline & & $\mathrm{Cr}$ & 16 & 1 & $\mathbf{U}$ & $37 \pm 4$ & 22 \\
\hline & & $\mathrm{Fe}$ & $26.3 \pm 0.1 \%$ & 3 & $\mathrm{v}$ & 82 & 1 \\
\hline & & $\mathbf{K}$ & $600 \pm 70$ & 4 & $\mathrm{Zn}$ & 785 & 1 \\
\hline \multirow[t]{11}{*}{80.00577} & USGS GXR-2 & $\mathrm{Ag}$ & $17.6 \pm 0.8$ & 12 & $\mathrm{La}$ & 28 & 1 \\
\hline & & Al & $18.5 \%$ & 2 & $\mathbf{L i}$ & 38 & 2 \\
\hline & & As & 37 & 2 & Mn & 960 & 2 \\
\hline & & Cd & 4.6 & 2 & $\mathrm{Na}$ & 5500 & 2 \\
\hline & & $\mathrm{Ce}$ & 50 & 2 & $\mathbf{R b}$ & 67 & 2 \\
\hline & & $\mathrm{Cr}$ & 34 & 2 & $\mathrm{Sm}$ & 3.5 & 1 \\
\hline & & Cs & 5.4 & 2 & Th & $8.6 \pm 0.3$ & 5 \\
\hline & & Dy & 3.0 & 2 & $\mathbf{U}$ & $3.0 \pm 0.1$ & 3 \\
\hline & & Eu & 0.74 & 2 & V & 65 & 1 \\
\hline & & $\mathbf{F e}$ & $1.83 \%$ & 2 & w & 1.4 & 2 \\
\hline & & $\mathbf{H f}$ & 8.7 & 2 & $\mathbf{Y b}$ & 1.8 & 2 \\
\hline \multirow[t]{2}{*}{80.00579} & USGS GXR-3 & As & 4300 & 2 & $\mathrm{Hg}$ & $0.34 \pm 0.03$ & 5 \\
\hline & & Cd & 0.53 & 2 & $\mathrm{Li}$ & 122 & 2 \\
\hline
\end{tabular}




\begin{tabular}{|c|c|c|c|c|c|c|c|}
\hline $\begin{array}{l}\text { H-8 } \\
\text { No. } \\
\end{array}$ & $\begin{array}{c}\text { Agency } \\
\text { ID }\end{array}$ & Element & $\begin{array}{c}\text { Mean Concentration } \\
\pm \text { Standard Deviation } \\
\text { (ppm uniess } \\
\text { otherwise indicated) }\end{array}$ & $\begin{array}{c}\text { No. of } \\
\text { Determinations }\end{array}$ & Element & $\begin{array}{c}\text { Mean Concentration } \\
\pm \text { Standard Deviation } \\
\text { (ppm unless } \\
\text { otherwise indicated) }\end{array}$ & $\begin{array}{c}\text { No. of } \\
\text { Determinations }\end{array}$ \\
\hline \multirow[t]{3}{*}{80.00581} & USGS GXR-4 & As & 107 & 2 & Th & $21.4 \pm 0.5$ & 3 \\
\hline & & Cd & 0.67 & 2 & $\mathrm{U}$ & $6.1 \pm 0.1$ & 3 \\
\hline & & $\mathrm{Li}$ & 8 & 2 & W & 30 & 2 \\
\hline \multirow[t]{15}{*}{80.00583} & USGS GXR-5 & Al & $19.7 \pm 0.6$ & 9 & Mo & $32 \pm 2$ & 3 \\
\hline & & As & $12 \pm 1$ & 5 & $\mathrm{Na}$ & $7400 \pm 600$ & 12 \\
\hline & & $\mathrm{Ba}$ & $1880 \pm 160$ & 10 & $\mathrm{Rb}$ & $41 \pm 3$ & 10 \\
\hline & & $\mathrm{Br}$ & 8.2 & 2 & $\mathrm{Sc}$ & $7.4 \pm 0.5$ & 10 \\
\hline & & $\mathrm{Ce}$ & $40 \pm 2$ & 12 & $\mathrm{Se}$ & 1.1 & 1 \\
\hline & & Co & $29 \pm 2$ & 13 & $\mathrm{Sm}$ & $3.2 \pm 0.2$ & 13 \\
\hline & & $\mathrm{Cr}$ & $100 \pm 4$ & 16 & $\mathrm{Sr}$ & 104 & 2 \\
\hline & & Cs & $2.4 \pm 0.2$ & 6 & $\mathrm{Ta}$ & $0.46 \pm 0.04$ & 6 \\
\hline & & Dy & 2.7 & I & Th & $5.3 \pm 0.2$ & 12 \\
\hline & & $\mathrm{Eu}$ & $0.96 \pm 0.07$ & 12 & $\mathrm{Ti}$ & 2280 & 2 \\
\hline & & $\mathrm{Fe}$ & $3.21 \pm 0.17 \%$ & 17 & $\mathrm{U}$ & $2.0 \pm 0.1$ & 4 \\
\hline & & Hf & $5.0 \pm 0.4$ & 6 & $\mathrm{v}$ & $76 \pm 22$ & 9 \\
\hline & & $\mathbf{K}$ & $8100 \pm 900$ & 4 & $\mathrm{Yb}$ & $1.9 \pm 0.3$ & 8 \\
\hline & & $\mathrm{La}$ & $20 \pm 2$ & 11 & $\mathrm{Zn}_{\mathbf{n}}$ & 31 & 1 \\
\hline & & $\mathbf{M n}$ & $290 \pm 30$ & 7 & & & \\
\hline \multirow[t]{9}{*}{80.00585} & USGS GXR-6 & $\mathrm{Al}$ & $16.5 \pm 0.8 \%$ & 11 & Mn & $1080 \pm 60$ & 9 \\
\hline & & As & $340 \pm 20$ & 9 & $\mathrm{Na}$ & $1010 \pm 90$ & 13 \\
\hline & & Ba & $1300 \pm 180$ & 10 & $\mathrm{Rb}$ & $104 \pm 9$ & 7 \\
\hline & & $\mathrm{Ce}$ & $38 \pm 2$ & 10 & Sc & $28 \pm 1$ & 10 \\
\hline & & Co & $14 \pm 1$ & 12 & $\mathrm{Sm}$ & $2.7 \pm 0.3$ & 12 \\
\hline & & $\mathrm{Cr}$ & $100 \pm 13$ & 12 & $\mathrm{Sr}$ & 37 & 2 \\
\hline & & Cs & $4.4 \pm 0.4$ & 4 & $\mathrm{Ta}$ & $0.46 \pm 0.15$ & 6 \\
\hline & & $\mathrm{Eu}$ & $0.74 \pm 0.09$ & 10 & Th & $5.3 \pm 0.4$ & 10 \\
\hline & & $\mathrm{Fe}$ & $5.5 \pm 0.3 \%$ & 12 & $\mathrm{Ti}$ & $4900 \pm 200$ & 5 \\
\hline
\end{tabular}


TABLE C-I (cont)

H-8

Asency

ID

\begin{tabular}{|c|c|}
\hline 80.00594 & CCRMP SO-1 \\
\hline 80.00596 & CCRMP SO-2 \\
\hline 80.00598 & CCRMP SO-3 \\
\hline 80.00600 & CCRMP SO-4 \\
\hline 80.00605 & NBS 1632A \\
\hline 80.00611 & USGS MAG-1 \\
\hline 80.00613 & USGS RGM-1 \\
\hline 80.00632 & NBS 1569 \\
\hline 80.00633 & NBA 1633A \\
\hline
\end{tabular}

Mean Concentration

\pm Standard Deviation

(ppm unkews

Element

otherwise indicated)

Ga

Hf

$\mathrm{Hg}$

K

La

B
Sr

U

\section{B}

B

B

Cl

Cl

U

Al

As

B

$\mathrm{Ba}$

$\mathrm{Ca}$

$\mathrm{Ce}$

$\begin{aligned} 33 & \\ 4.2 & \pm 0.4 \\ 0.08 & \pm 0.01 \\ 1.99 & \pm 0.17 \% \\ 15 & \pm 1\end{aligned}$

$17 \pm 2$

330

$1.0 \pm 0.1$

$24 \pm 2$

$42 \pm 3$

$55 \pm 4$

$3.08 \%$

$530 \pm 50$

$0.47 \pm 0.05$

$14.2 \pm 0.4 \%$

$145 \pm 8$

$39 \pm 3$

$1450 \pm 110$

$1.1 \pm 0.1 \%$

$180 \pm 5$
No. of

Determinations

2

4

4

7

12

5

1

3

6

4

8

1

11

4

12

12

3

6

3

6
Mean Concentration

\pm Standard Deviation

(ppm unies:

Element

otherwise indiented)

No. of

Determinution:

$\begin{array}{ll}\mathrm{U} & 1.5 \pm 0.1\end{array}$

V $\quad 198 \pm 12$

$\mathrm{Yb} \quad 2.6 \pm 0.3$

$\mathrm{Zn} \quad 158$

3

13

6

5000

$1.7 \pm 0.1$

2

3

U

U

$1.1 \pm 0.1$

3

U

$2.4 \pm 0.1$

15

U

$1.3 \pm 0.1$

3

$\mathrm{Mn}$

$210 \pm 50$

21

$\mathrm{Na}$

$1740 \pm 100$

$134 \pm 16$

$41 \pm 2$

$10.7 \pm 0.8$

$\mathrm{Se}$

$14.5 \pm 1.3$

Sm 


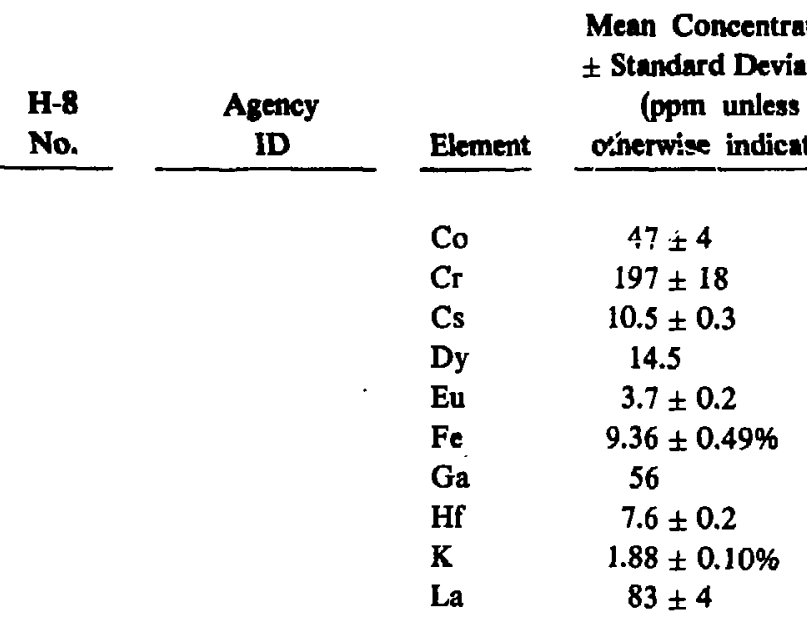

No. of

Determinations

Element

24

25

7

2

9

23

2

5

10

19

$\mathrm{Yb}$
Mean Concentration \pm Standard Deviation

(ppm unless

No. of otherwise indicated) Determinations

$$
\begin{gathered}
770 \\
1.8 \pm 0.2 \\
2.8 \pm 0.5 \\
24.6 \pm 0.9 \\
8000 \pm 600 \\
10.6 \pm 0.4 \\
294 \pm 28 \\
5.4 \pm 0.4 \\
8.2
\end{gathered}
$$

2

4

4

21

7

10

12 
TABLE C-II

ELEMENTAL CONCENTRATIONS IN QUALITY ASSURANCE

SAMPLE MATERIALS MEASURED BY H-8 DURING 1980

NUMBER

ANALYSIS RESULT UNCERTAINTY

80.00500

UNITS

COMPLETION

DATE

80.00500

TDS

TDS

TDS

TDS

TDS

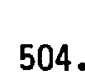

50.

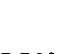

48.

PPM
PPM

472 .

47.

PPM

486.

526.

49.

PPM

52. PPM

520.

52.

PPM

$04 / 07 / 80$

$04 / 07 / 80$

$04 / 07 / 80$

$04 / 07 / 80$

$04 / 07 / 80$

$04 / 07 / 80$

80.00501

COND

1120.

112.

UMHO*

$08 / 25 / 80$

80.00503

$\begin{array}{ll}F & .4 \\ F & .4 \\ F & .33 \\ F & .31 \\ \text { N03 } & .97\end{array}$

$.04 \quad$ PPM

.04 PPM

.03 PPM

.03 PPM

.1 PPM

$06 / 02 / 80$

$06 / 02 / 80$

$07 / 01 / 80$

$07 / 01 / 80$

$06 / 02 / 80$

80.00504

$\begin{array}{lr}F & 2.3 \\ F & 2.3 \\ F & 2.3 \\ F & 2 . \\ F & 2.1 \\ F & 2.1 \\ F & 2.1 \\ \text { N03 } & 44.4 \\ \text { N03 } & 44.9\end{array}$

.2

.2

.2

.2

.2

.2

.2

4.4

4.5

PPM
PPM
PPM
PPM
PPM
PPM
PPM
PPM
PPM

$06 / 02 / 80$

$06 / 02 / 80$

$06 / 11 / 80$

$07 / 01 / 80$

$07 / 29 / 80$

$07 / 30 / 80$

$07 / 31 / 80$

$06 / 02 / 80$

$06 / 11 / 80$

80.00505

AG

AS

AS

AS

AS

AS

AS

AS

AS

AS

AS

AS

AS

AS

AS

AS

AS

\section{2}

3.2

77.

76.

73.

84.

67.

79.

110 .

72.

72.

81.

79.

68 .

69.

79.

$\cdot$

.3

PPB

PPB

PPB

PPB

3. $\quad P P B$

3. $\quad \mathrm{PPB}$

3. $P P B$

3. $\quad$ PPB

.8 PPB

.8 PPB

7. $\quad \mathrm{PPB}$

8. PPB

8. $P P B$

3. $\mathrm{PPB}$

7. $P P B$

8. $P P B$

7. $P P B$

69.

68.

79.

3. $P P B$

PPB
PPB

$06 / 12 / 80$

$06 / 12 / 80$

$05 / 27 / 80$

$05 / 27 / 80$

$05 / 27 / 80$

$05 / 27 / 80$

$05 / 27 / 80$

$05 / 27 / 80$

$05 / 28 / 80$

$05 / 28 / 80$

$06 / 02 / 80$

$06 / 02 / 80$

$06 / 02 / 80$

$06 / 02 / 80$

$06 / 02 / 80$

$06 / 02 / 80$

$06 / 02 / 80$

$06 / 02 / 80$

8. PPB $06 / 02 / 80$ 
TABLE C-II (cont)

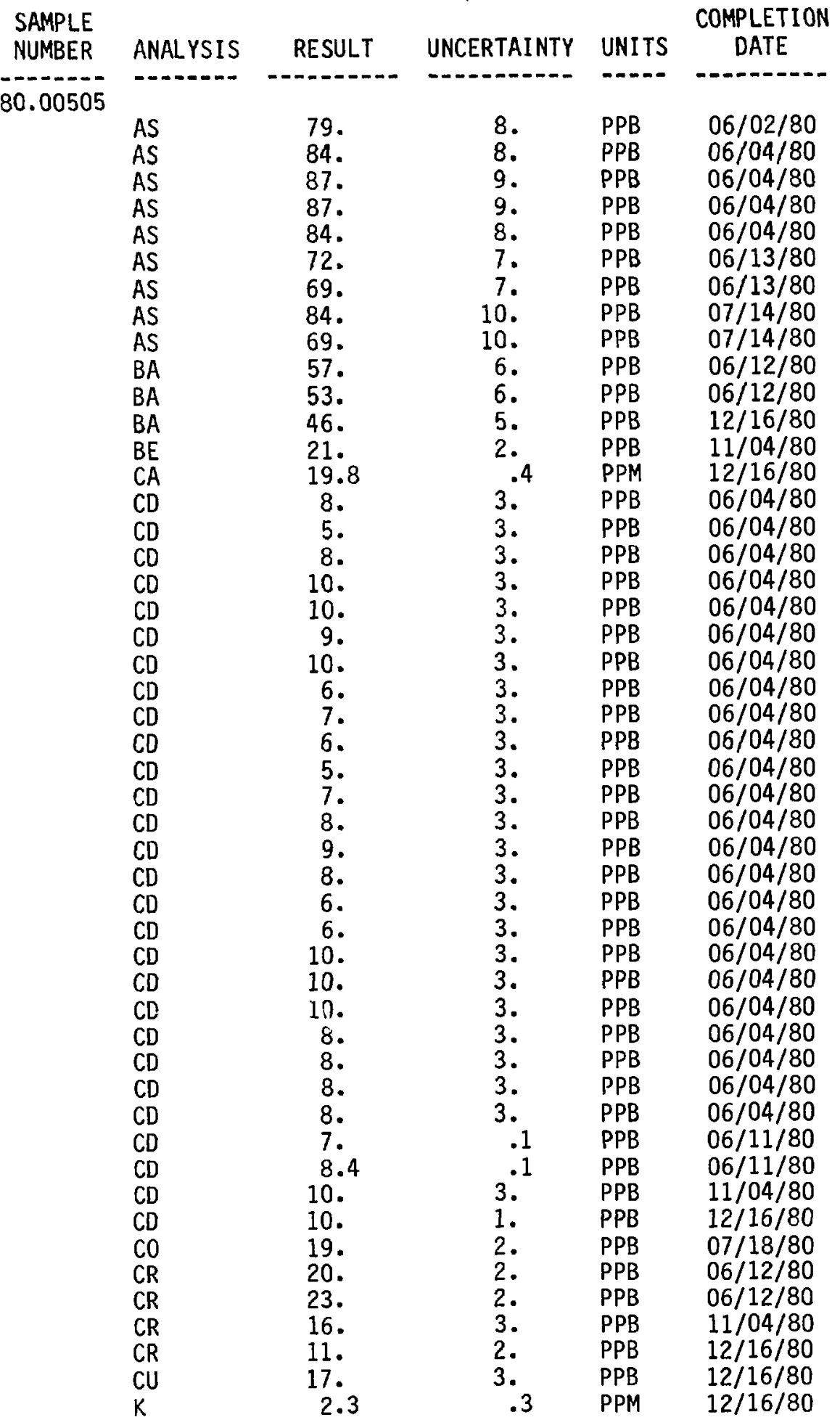


TABLE C-II (cont)

\begin{tabular}{clcccc}
$\begin{array}{c}\text { SAMPLE } \\
\text { NUMBER }\end{array}$ & ANALYSIS & RESULT & UNCERTAINTY & UNITS & COMPLETION \\
\hline $\mathbf{D A T E}$ & \\
\hline 80.00505 & & & & & \\
& MG & 8.1 & .8 & PPM & $12 / 16 / 80$ \\
& MO & 110. & 20. & PPB & $07 / 14 / 80$ \\
& NA & 9.3 & .3 & PPM & $12 / 16 / 80$ \\
& NI & 52. & 11. & PPB & $07 / 18 / 80$ \\
& NI & 67. & 12. & PPB & $07 / 18 / 80$ \\
& PB & 23. & 2. & PPB & $06 / 13 / 80$ \\
& PB & 27. & 2. & PPB & $06 / 13 / 80$ \\
& PB & 28. & 5. & PPB & $12 / 16 / 80$ \\
& SE & 11. & 5. & PPB & $06 / 13 / 80$ \\
& SE & 12. & 5. & PPB & $06 / 13 / 80$ \\
& SE & 13. & 3. & PPB & $08 / 20 / 80$ \\
& ZN & 60. & 3. & PPB & $12 / 16 / 80$
\end{tabular}

80.00510

$\begin{array}{lrlll}\mathrm{H}-3 & 1100 . & 300 . & \mathrm{PCI} / \mathrm{L} & 03 / 10 / 80 \\ \mathrm{H}-3 & 900 . & 300 . & \mathrm{PCI} / \mathrm{L} & 03 / 18 / 80 \\ \mathrm{H}-3 & 500 . & 300 . & \mathrm{PCI} / \mathrm{L} & 04 / 03 / 80 \\ \mathrm{H}-3 & 900 . & 300 . & \mathrm{PCI} / \mathrm{L} & 04 / 15 / 80 \\ \mathrm{H}-3 & 700 . & 300 . & \mathrm{PCI} / \mathrm{L} & 04 / 18 / 80 \\ \mathrm{H}-3 & 800 . & 300 . & \mathrm{PCI} / \mathrm{L} & 05 / 02 / 80 \\ \mathrm{H}-3 & 800 . & 300 . & \mathrm{PCI} / \mathrm{L} & 05 / 19 / 80 \\ \mathrm{H}-3 & 1100 . & 300 . & \mathrm{PCI} / \mathrm{L} & 06 / 10 / 80 \\ \mathrm{H}-3 & 1200 . & 300 . & \mathrm{PCI} / \mathrm{L} & 10 / 17 / 80 \\ \mathrm{H}-3 & 1000 . & 300 . & \mathrm{PCI} / \mathrm{L} & 11 / 04 / 80 \\ \mathrm{H}-3 & 1500 . & 300 . & \mathrm{PCI} / \mathrm{L} & 11 / 19 / 80 \\ \mathrm{H}-3 & 1700 . & 300 . & \mathrm{PCI} / \mathrm{L} & 12 / 03 / 80 \\ \mathrm{H}-3 & \mathbf{H}-300 . & 300 . & \mathrm{PCI} / \mathrm{L} & 12 / 23 / 80\end{array}$

80.00511

$\begin{array}{lllll}H-3 & 1800 . & 400 . & \mathrm{PCI} / \mathrm{L} & 01 / 15 / 80 \\ \mathrm{H}-3 & 1800 . & 400 . & \mathrm{PCI} / \mathrm{L} & 01 / 15 / 80 \\ \mathrm{H}-3 & 2000 . & 400 . & \mathrm{PCI} / \mathrm{L} & 01 / 15 / 80 \\ \mathrm{H}-3 & 1800 . & 400 . & \mathrm{PCI} / \mathrm{L} & 01 / 15 / 80 \\ \mathrm{H}-3 & 2300 . & 400 . & \mathrm{PCI} / \mathrm{L} & 01 / 15 / 80 \\ \mathrm{H}-3 & 1800 . & 400 . & \mathrm{PCI} / \mathrm{L} & 01 / 15 / 80 \\ \mathrm{H}-3 & 1900 . & 400 . & \mathrm{PCI} / \mathrm{L} & 01 / 28 / 80 \\ \mathrm{H}-3 & 1500 . & 400 . & \mathrm{PCI} / \mathrm{L} & 02 / 04 / 80 \\ \mathrm{H}-3 & 2000 . & 400 . & \mathrm{PCI} / \mathrm{L} & 02 / 20 / 80 \\ \mathrm{H}-3 & 1900 . & 400 . & \mathrm{PCI} / \mathrm{L} & 02 / 28 / 80 \\ \mathrm{H}-3 & 2000 . & 400 . & \mathrm{PCI} / \mathrm{L} & 03 / 10 / 80 \\ \mathrm{H}-3 & 1500 . & 300 . & \mathrm{PCI} / \mathrm{L} & 03 / 18 / 80 \\ \mathrm{H}-3 & 1600 . & 400 . & \mathrm{PCI} / \mathrm{L} & 04 / 03 / 80 \\ \mathrm{H}-3 & 1700 . & 400 . & \mathrm{PCI} / \mathrm{L} & 04 / 15 / 80 \\ \mathrm{H}-3 & 2300 . & 400 . & \mathrm{PCI} / \mathrm{L} & 04 / 18 / 80 \\ \mathrm{H}-3 & 1700 . & 300 . & \mathrm{PCI} / \mathrm{L} & 05 / 02 / 80 \\ \mathrm{H}-3 & 1400 . & 300 . & \mathrm{PCI} / \mathrm{L} & 05 / 19 / 80 \\ \mathrm{H}-3 & 1800 . & 300 . & \mathrm{PCI} / \mathrm{L} & 06 / 10 / 80 \\ \mathrm{H}-3 & 2500 . & 300 . & \mathrm{PCI} / \mathrm{L} & 10 / 17 / 80 \\ \mathrm{H}-3 & 1800 . & 300 . & \mathrm{PCI} / \mathrm{L} & 11 / 04 / 80\end{array}$


TABLE C-Il (cont)

SAMPLE NUMBER 80.00511

$\mathrm{H}-3$

$\mathrm{H}-3$

H-3

80.00512

$\mathrm{H}-3$

H-3

H-3

H-3

H-3

$\mathrm{H}-3$

H-3

$\mathrm{H}-3$

$\mathrm{H}-3$

$\mathrm{H}-3$

$\mathrm{H}-3$

$\mathrm{H}-3$

$\mathrm{H}-3$

H-3

H-3

$\mathrm{H}-3$

H-3

H-3

H-3

$\mathrm{H}-3$

$\mathrm{H}-3$

$\mathrm{H}-3$

H-3

80.00513

$\mathrm{H}-3$

$\mathrm{H}-3$

$\mathrm{H}-3$

$\mathrm{H}-3$

$\mathrm{H}-3$

$\mathrm{H}-3$

$\mathrm{H}-3$

$\mathrm{H}-3$

H-3

$\mathrm{H}-3$

H-3

H-3

H-3

H-3

$\mathrm{H}-3$

H-3

$\mathrm{H}-3$

$\mathrm{H}-3$

$\mathrm{H}-3$

4300.
3400
4000
3700
3600
3400
3600
3600
3900
4000
3900
4000
3300
3400
3800
3100
2800
3100
3600
3500
3600
3600
3400

1300.

1900.

1900.
COMPLETION

DATE

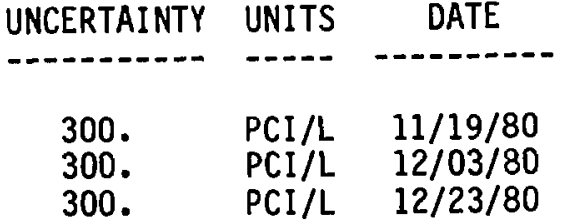

400. PCI/L $01 / 15 / 80$

400. PCI/L $01 / 15 / 80$

400. PCI/L $01 / 15 / 80$

400. $\mathrm{PCI} / \mathrm{L} \quad 01 / 15 / 80$

400. PCI/L $01 / 15 / 80$

400. PCI/L $01 / 15 / 80$

400. PCI/L $01 / 28 / 80$

400. PCI/L 02/04/80

400. PCI/L $02 / 20 / 80$

400. PCI/L $02 / 28 / 80$

400. PCI/L 03/10/80

400. PCI/L $03 / 18 / 80$

400. PCI/L $04 / 03 / 80$

400. PCI/L $04 / 15 / 80$

400. PCI/L $04 / 18 / 80$

300. PCI/L $05 / 02 / 80$

300. PCI/L $05 / 19 / 80$

300. PCI/L $06 / 10 / 80$

300. PCI/L $10 / 17 / 80$

300. PCI/L $11 / 04 / 80$

300. PCI/L $11 / 19 / 80$

300. PCI/L $12 / 03 / 80$

300. PCI/L $12 / 23 / 80$

400. PCI/L $01 / 15 / 80$

400. PCI/L $01 / 15 / 80$

400. PCI/L $01 / 15 / 80$

400. PCI/L $01 / 15 / 80$

400. PCI/L $01 / 15 / 80$

400. PCI/L $01 / 15 / 80$

400. PCI/L $01 / 28 / 80$

400. PCI/L $02 / 04 / 80$

400. PCI/L $02 / 20 / 80$

400. $\quad \mathrm{PCI} / \mathrm{L} \quad 02 / 28 / 80$

400. $\quad \mathrm{PCI} / \mathrm{L} \quad 03 / 10 / 80$

400. PCI/L $03 / 18 / 80$

400. PCI/L $04 / 03 / 80$

400. PCI/L $04 / 15 / 80$

400. PCI/L $04 / 18 / 80$

400. PCI/L 05/02/80

400. PCI/L $05 / 19 / 80$

400. PCI/L $06 / 10 / 80$

400. PCI/L $10 / 17 / 80$ 
TABLE C-II (cont)

\begin{tabular}{|c|c|c|c|c|c|}
\hline $\begin{array}{l}\text { SAMPLE } \\
\text { NUMBER }\end{array}$ & ANALYSIS & RESULT & UNCERTAINTY & UNITS & $\begin{array}{l}\text { COMPLETION } \\
\text { DATE }\end{array}$ \\
\hline \multicolumn{6}{|l|}{80.00513} \\
\hline & $\begin{array}{l}H-3 \\
H-3 \\
H-3 \\
H-3\end{array}$ & $\begin{array}{l}8100 . \\
8500 . \\
8300 . \\
9000 .\end{array}$ & $\begin{array}{l}400 . \\
400 . \\
400 . \\
400 .\end{array}$ & $\begin{array}{l}\mathrm{PCI} / \mathrm{L} \\
\mathrm{PCI} / \mathrm{L} \\
\mathrm{PCI} / \mathrm{L} \\
\mathrm{PCI} / \mathrm{L}\end{array}$ & $\begin{array}{l}11 / 04 / 80 \\
11 / 19 / 80 \\
12 / 03 / 80 \\
12 / 23 / 80\end{array}$ \\
\hline \multicolumn{6}{|l|}{80.00514} \\
\hline & $\begin{array}{l}H-3 \\
H-3 \\
H-3 \\
H-3 \\
H-3 \\
H-3 \\
H-3 \\
y-3 \\
H-3 \\
H-3 \\
H-3 \\
H-3 \\
H-3 \\
H-3 \\
H-3 \\
H-3 \\
H-3 \\
H-3\end{array}$ & $\begin{array}{l}20100 . \\
18900 . \\
19000 . \\
19300 . \\
18500 . \\
20100 . \\
19000 . \\
18500 . \\
18600 . \\
19500 . \\
18400 . \\
17700 . \\
17500 . \\
18300 . \\
18400 . \\
16500 . \\
16000 . \\
16600 .\end{array}$ & $\begin{array}{l}600 . \\
600 . \\
600 . \\
600 . \\
600 . \\
600 . \\
600 . \\
600 . \\
600 . \\
400 . \\
500 . \\
500 . \\
500 . \\
500 . \\
600 . \\
500 . \\
500 . \\
500 .\end{array}$ & $\begin{array}{l}P C I / L \\
P C I / L \\
P C I / L \\
P C I / L \\
P C I / L \\
P C I / L \\
P C I / L \\
P C I / L \\
P C I / L \\
P C I / L \\
P C I / L \\
P C I / L \\
P C I / L \\
P C I / L \\
P C I / L \\
P C I / L \\
P C I / L \\
P C I / L\end{array}$ & $\begin{array}{l}01 / 15 / 80 \\
01 / 15 / 80 \\
01 / 15 / 80 \\
01 / 15 / 80 \\
01 / 15 / 80 \\
01 / 15 / 80 \\
01 / 28 / 80 \\
02 / 04 / 80 \\
02 / 20 / 80 \\
02 / 28 / 80 \\
03 / 10 / 80 \\
03 / 18 / 80 \\
04 / 03 / 80 \\
04 / 15 / 80 \\
04 / 18 / 80 \\
05 / 02 / 80 \\
05 / 19 / 80 \\
06 / 10 / 80\end{array}$ \\
\hline \multicolumn{6}{|c|}{80.00515} \\
\hline & $\begin{array}{l}\text { B } \\
\text { TH } \\
\text { TH } \\
\text { TH } \\
\text { TH } \\
\text { TH } \\
\text { TH } \\
\text { TH } \\
\text { TH } \\
\text { TH } \\
\text { TH } \\
\text { TH } \\
\text { TH } \\
\text { TH } \\
\text { TH } \\
\text { TH }\end{array}$ & $\begin{array}{l}86 . \\
353 . \\
345 . \\
349 . \\
364 . \\
360 . \\
362 . \\
351 . \\
344 . \\
344 . \\
353 . \\
341 . \\
346 . \\
342 . \\
348 . \\
347 .\end{array}$ & $\begin{array}{l}9 . \\
15 . \\
15 . \\
15 . \\
15 . \\
15 . \\
15 . \\
15 . \\
15 . \\
15 . \\
15 . \\
15 . \\
15 . \\
15 . \\
15 . \\
15 .\end{array}$ & $\begin{array}{l}\text { PPM } \\
\text { PPM } \\
\text { PPM } \\
\text { PPM } \\
\text { PPM } \\
\text { PPM } \\
\text { PPM } \\
\text { PPM } \\
\text { PPM } \\
\text { PPM } \\
\text { PPM } \\
\text { PPM } \\
\text { PPM } \\
\text { PPM } \\
\text { PPM } \\
\text { PPM }\end{array}$ & $\begin{array}{l}12 / 23 / 80 \\
02 / 01 / 80 \\
02 / 01 / 80 \\
02 / 01 / 80 \\
02 / 10 / 80 \\
02 / 10 / 80 \\
02 / 10 / 80 \\
02 / 20 / 80 \\
02 / 20 / 80 \\
02 / 20 / 80 \\
03 / 01 / 80 \\
03 / 01 / 80 \\
03 / 01 / 80 \\
03 / 10 / 80 \\
03 / 10 / 80 \\
03 / 10 / 80\end{array}$ \\
\hline
\end{tabular}

80.00517

$\begin{array}{lllll}C L & 140 . & 30 . & \text { PPM } & 01 / 22 / 80 \\ C L & 160 . & 30 . & \text { PPM } & 01 / 22 / 80 \\ \text { SR } & 250 . & 30 . & \text { PPM } & 12 / 24 / 80\end{array}$


TABLE C-II (cont)

\begin{tabular}{|c|c|c|c|c|c|}
\hline $\begin{array}{l}\text { SAMPLE } \\
\text { NUMBER }\end{array}$ & ANALYSIS & RESULT & UNCERTAINTY & UNITS & $\begin{array}{l}\text { COMPLETION } \\
\text { DATE }\end{array}$ \\
\hline - & 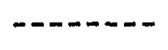 & $-\cdots-\cdots-n$ & 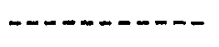 & ---- & $-\cdots-\cdots \cdots$ \\
\hline & $R A-226$ & 73. & 2. & $P C I / G$ & $03 / 01 / 80$ \\
\hline & $R A-226$ & 75. & 2. & $P C I / G$ & $03 / 01 / 80$ \\
\hline & $R A-226$ & 72. & 3. & $P C I / G$ & $06 / 15 / 80$ \\
\hline & U & 215. & 20. & PPM & $01 / 02 / 80$ \\
\hline & U & 211. & 20. & PPM & $01 / 02 / 80$ \\
\hline & U & 220 . & 20. & PPM & $01 / 17 / 80$ \\
\hline & $U$ & 230. & 20. & PPM & $01 / 17 / 80$ \\
\hline & U & 230. & 20. & PPM & $01 / 18 / 80$ \\
\hline & $U$ & 220. & 20. & PPM & $01 / 18 / 80$ \\
\hline & U & 220. & 20 . & PPM & $01 / 18 / 80$ \\
\hline & $U$ & 212 . & 20. & PPM & $05 / 19 / 80$ \\
\hline & $U$ & 212 . & 20. & PPM & $05 / 19 / 80$ \\
\hline & U & 212 . & 20. & PPM & $05 / 19 / 80$ \\
\hline & $U$ & 212 . & 20. & PPM & $05 / 19 / 80$ \\
\hline & $U$ & 210 . & 20. & PPM & $05 / 19 / 80$ \\
\hline & U & 217 . & 20. & PPM & $05 / 22 / 80$ \\
\hline & $U$ & 211 . & 20. & PPM & $05 / 22 / 80$ \\
\hline & $\mathrm{U}$ & 221. & 10. & PPM & $06 / 19 / 80$ \\
\hline & U & 218 . & 10. & PPM & $06 / 19 / 80$ \\
\hline & U & 220 . & 10. & PPM & $06 / 19 / 80$ \\
\hline & U & 219. & 10. & PPM & $06 / 19 / 80$ \\
\hline & $U$ & 219. & 10. & PPM & $06 / 19 / 80$ \\
\hline & U & 219 & 10. & PPM & $06 / 19 / 80$ \\
\hline & U & 214.5 & 10. & PPM & $07 / 08 / 80$ \\
\hline & U & 215. & 10. & PPM & $07 / 08 / 80$ \\
\hline & U & 217.7 & 10. & PPM & $07 / 08 / 80$ \\
\hline & U & 223. & 25. & PPM & $07 / 14 / 80$ \\
\hline & $U$ & 250. & 25. & PPM & $07 / 14 / 80$ \\
\hline & U & 214. & 25 . & PPM & $07 / 14 / 80$ \\
\hline & U & 234. & 25 . & PPM & $07 / 14 / 80$ \\
\hline & $U$ & 217 . & 20. & PPM & $08 / 18 / 90$ \\
\hline & U & 219. & 20 & PPM & $08 / 18 / 80$ \\
\hline & $U$ & 210 & 20 & PPM & $10 / 08 / 80$ \\
\hline & U & 215 . & 20. & PPM & $10 / 08 / 80$ \\
\hline & U & 219. & 20. & PPM & $10 / 15 / 80$ \\
\hline & U & 217. & 20. & PPM & $11 / 19 / 80$ \\
\hline & U & 215 . & 20. & PPM & $12 / 09 / 80$ \\
\hline & U & 220 . & 20. & PPM & $12 / 09 / 80$ \\
\hline
\end{tabular}

80.00519

$\begin{array}{lllll}\text { RA-226 } & 1580 . & 50 . & \text { PCI /G } & 06 / 15 / 80 \\ U & 4402 . & 220 . & \text { PPM } & 12 / 04 / 80 \\ U & 4530 . & 230 . & P P M & 12 / 04 / 80 \\ U & 4440 . & 230 . & P P M & 12 / 04 / 80 \\ U & 4527 . & 220 . & P P M & 12 / 05 / 80 \\ U & 4516 . & 220 . & P P M & 12 / 05 / 80 \\ U & 4529 . & 220 . & P P M & 12 / 05 / 80\end{array}$


TABLE C-II (cont)

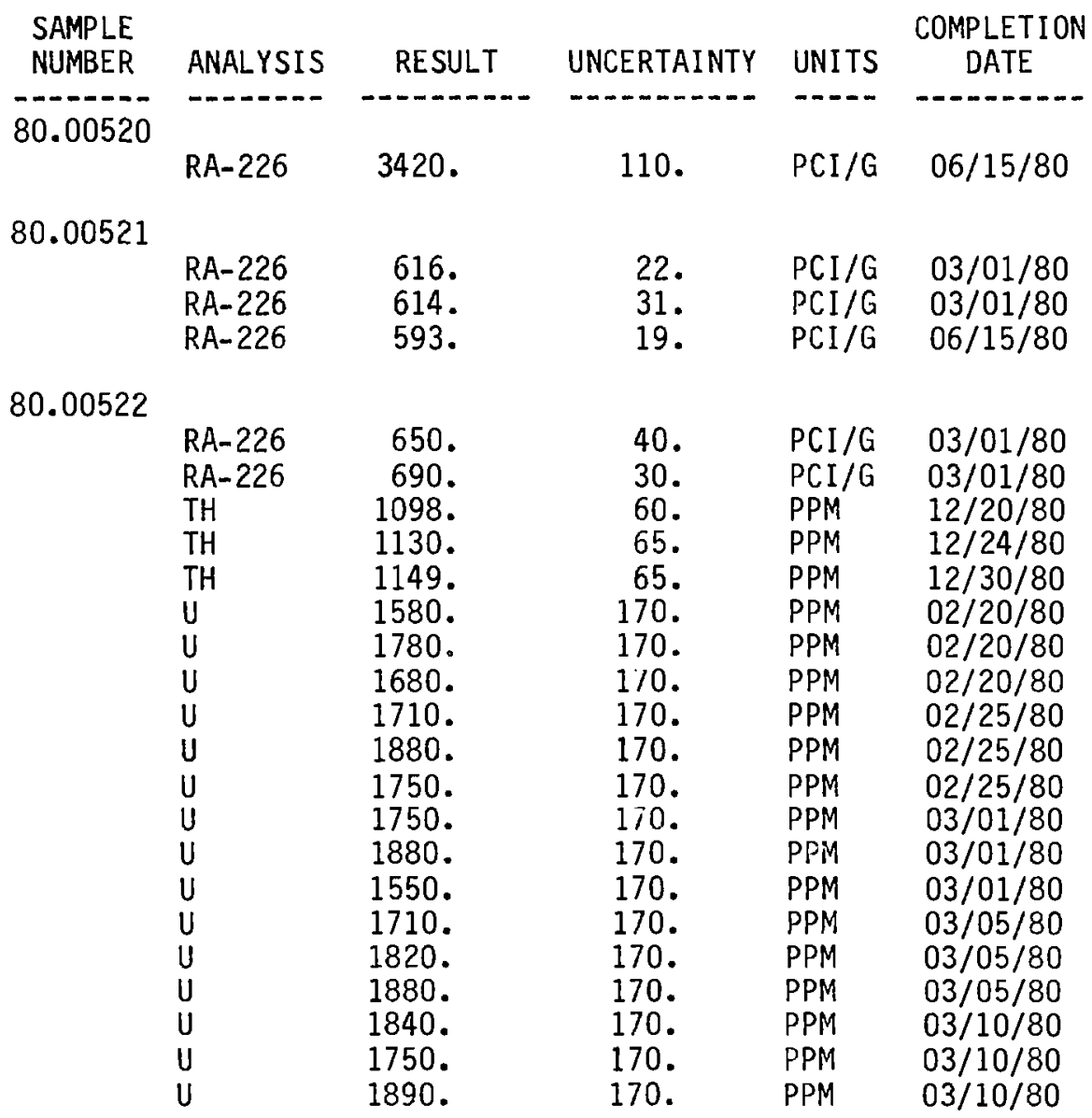

80.00523

$\begin{array}{lllll}\text { TH } & 72.2 & 4.4 & \text { PPM } & 12 / 20 / 80 \\ \text { TH } & 83.5 & 5.4 & \text { PPM } & 12 / 20 / 80 \\ \text { TH } & 89.7 & 5.2 & \text { PPM } & 12 / 24 / 80 \\ \text { TH } & 87.1 & 5.2 & \text { PPM } & 12 / 24 / 80 \\ \text { TH } & 82.6 & 4.9 & \text { PPM } & 12 / 30 / 80 \\ \text { TH } & 79.5 & 4.3 & \text { PPM } & 12 / 30 / 80 \\ U & 40 . & 4 . & \text { PPM } & 01 / 02 / 80 \\ U & 40 . & 4 . & P P M & 01 / 02 / 80 \\ U & 40.8 & 2 . & \text { PPM } & 01 / 12 / 80 \\ U & 41.7 & 2 . & P P M & 01 / 12 / 80 \\ U & 40.5 & 2 . & P P M & 01 / 12 / 80 \\ U & 40 . & 4 . & P P M & 01 / 17 / 80 \\ U & 40 . & 4 . & P P M & 01 / 17 / 80 \\ U & 40 . & 2 . & P P M & 01 / 18 / 80 \\ U & 40 . & .4 & \text { PPM } & 01 / 18 / 80 \\ U & 41 . & .4 & P P M & 01 / 18 / 80 \\ U & 41 . & .4 & \text { PPM } & 01 / 18 / 80 \\ U & 41 . & .4 & \text { PPM } & 01 / 18 / 80 \\ U & 40 . & 2 . & P P M & 01 / 18 / 80\end{array}$


TABLE C-II (cont)

\begin{tabular}{|c|c|c|c|c|c|}
\hline \multirow{3}{*}{$\begin{array}{l}\text { SAMPLE } \\
\text { NUMBER } \\
80.00523\end{array}$} & ANALYSIS & RESULT & UNCERTAINTY & UNITS & $\begin{array}{l}\text { COMPLETION } \\
\text { DATE }\end{array}$ \\
\hline & $\cdots$ & 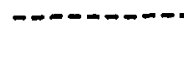 & 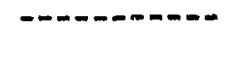 & $-\ldots-\infty$ & $\cdots$ \\
\hline & U & 42.3 & 2. & PPM & $01 / 18 / 80$ \\
\hline & $u$ & 41.2 & 2. & PPM & $01 / 18 / 80$ \\
\hline & U & 40.6 & 4. & PPM & $02 / 25 / 80$ \\
\hline & u & 36.2 & 4. & PPM & $02 / 25 / 80$ \\
\hline & u & 46.8 & 4. & PPM & $02 / 25 / 80$ \\
\hline & U & 44.7 & 4. & PPM & $03 / 01 / 80$ \\
\hline & U & 41.3 & 4. & PPM & $03 / 01 / 80$ \\
\hline & U & 42.3 & 4. & PPM & $03 / 01 / 80$ \\
\hline & u & 38. & 4. & PPM & $03 / 05 / 80$ \\
\hline & u & 41.5 & 4. & PPM & $03 / 05 / 80$ \\
\hline & u & 43.1 & 4. & PPM & $03 / 05 / 80$ \\
\hline & $u$ & 48.2 & 4. & PPM & $03 / 10 / 80$ \\
\hline & u & 39.2 & 4. & PPM & $03 / 10 / 80$ \\
\hline & U & 38.3 & 4. & PPM & $03 / 10 / 80$ \\
\hline & $u$ & 40.4 & 4. & PPM & $03 / 31 / 80$ \\
\hline & u & 41.7 & 4. & PPM & $03 / 31 / 80$ \\
\hline & U & 40.4 & 4. & PPM & $03 / 31 / 80$ \\
\hline & u & 41.7 & 4. & PPM & $03 / 31 / 80$ \\
\hline & $u$ & 40.4 & 4. & PPM & $03 / 31 / 80$ \\
\hline & U & 41.7 & 4. & PPM & $03 / 31 / 80$ \\
\hline & $u$ & 39.9 & 4. & PPM & $04 / 01 / 80$ \\
\hline & U & 40.1 & 4. & PPM & $04 / 01 / 80$ \\
\hline & U & 30.5 & 4. & PPM & $04 / 01 / 80$ \\
\hline & u & 41.6 & 4. & PPM & $04 / 05 / 80$ \\
\hline & u & 37.8 & 4. & PPM & $04 / 05 / 80$ \\
\hline & U & 38.4 & 4. & PPM & $04 / 05 / 80$ \\
\hline & u & 38.3 & 4. & PPM & $04 / 10 / 80$ \\
\hline & U & 39. & 4. & PPM & $04 / 10 / 80$ \\
\hline & U & 40.1 & 4. & PPM & $04 / 10 / 80$ \\
\hline & U & 40.6 & 4. & PPM & $04 / 10 / 80$ \\
\hline & U & 38.2 & 4. & PPM & $04 / 15 / 80$ \\
\hline & $u$ & 41.3 & 4. & PPM & $04 / 15 / 80$ \\
\hline & u & 40.3 & 4. & PPM & $04 / 15 / 80$ \\
\hline & U & 43.3 & 4. & PPM & $04 / 20 / 80$ \\
\hline & U & 39.3 & 4. & PPM & $04 / 20 / 80$ \\
\hline & U & 38.6 & 4. & PPM & $04 / 20 / 80$ \\
\hline & U & 40.8 & 4. & PPM & $04 / 25 / 80$ \\
\hline & U & 35.9 & 4. & PPM & $04 / 25 / 80$ \\
\hline & U & 41.8 & 4. & PPM & $04 / 25 / 80$ \\
\hline & u & 40.6 & 4. & PPM & $04 / 25 / 80$ \\
\hline & U & 42.2 & 4. & PPM & $04 / 25 / 80$ \\
\hline & u & 37.4 & 4. & PPM & $04 / 25 / 80$ \\
\hline & u & 38. & 4. & PPM & $04 / 30 / 80$ \\
\hline & u & 42.1 & 4. & PPM & $04 / 30 / 80$ \\
\hline & u & 38.4 & 4. & PPM & $04 / 30 / 80$ \\
\hline & u & 34.1 & 4. & PPM & $04 / 30 / 80$ \\
\hline & u & 40.8 & 4. & PPM & $04 / 30 / 80$ \\
\hline & U & 0 & 4. & PPM & $05 / 19 / 80$ \\
\hline & & & & PPM & 05 \\
\hline
\end{tabular}


TABLE C-II (cont)

\begin{tabular}{|c|c|c|c|c|c|}
\hline $\begin{array}{l}\text { SAMPLE } \\
\text { NUMBER }\end{array}$ & ANALYSIS & RESULT & UNCERTAINTY & UNITS & $\begin{array}{l}\text { COMPLETION } \\
\text { DATE }\end{array}$ \\
\hline$-1-1=-1$ & 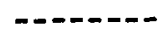 & 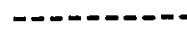 & $m-n-m-1$ & $\cdots$ & - \\
\hline 80.00023 & $U$ & 40. & 4. & PPM & $05 / 19 / 80$ \\
\hline & u & 40. & 4. & PPM & $05 / 19 / 80$ \\
\hline & U & 39. & 4. & PPM & $05 / 19 / 80$ \\
\hline & u & 39. & 4. & PPM & $05 / 19 / 80$ \\
\hline & $u$ & 41.1 & 4. & PPM & $05 / 29 / 80$ \\
\hline & U & 40.4 & 4. & PPM & $05 / 29 / 80$ \\
\hline & U & 38.8 & 4. & PPM & $05 / 29 / 80$ \\
\hline & $u$ & 41.6 & 4. & PPM & $05 / 29 / 80$ \\
\hline & U & 38.6 & 4. & PPM & $05 / 29 / 80$ \\
\hline & u & 40.7 & 4. & PPM & $05 / 29 / 80$ \\
\hline & U & 40. & 10. & PPM & $06 / 19 / 80$ \\
\hline & u & 41. & 10. & PPM & $06 / 19 / 80$ \\
\hline & U & 41. & 10. & PPM & $06 / 19 / 80$ \\
\hline & U & 40. & 10. & PPM & $06 / 19 / 80$ \\
\hline & U & 41. & 10. & PPM & $06 / 19 / 80$ \\
\hline & U & 41. & 10. & PPM & $06 / 19 / 80$ \\
\hline & U & 41. & 10. & PPM & $06 / 19 / 80$ \\
\hline & U & 40. & 10. & PPM & $06 / 19 / 80$ \\
\hline & U & 40.6 & 3. & PPM & $07 / 08 / 80$ \\
\hline & u & 41.3 & 3. & PPM & $07 / 08 / 80$ \\
\hline & U & 39. & 3. & PPM & $07 / 08 / 80$ \\
\hline & U & 38.8 & 3. & PPM & $07 / 08 / 80$ \\
\hline & U & 39. & 3. & PPM & $07 / 08 / 80$ \\
\hline & U & 41.2 & 4. & PPM & $08 / 18 / 80$ \\
\hline & $u$ & 40.6 & 4. & PPM & $08 / 18 / 80$ \\
\hline & U & 41.2 & 4. & PPM & $08 / 18 / 80$ \\
\hline & u & 41.5 & 4. & PPM & $08 / 18 / 80$ \\
\hline & $u$ & 41. & 4. & PPB & $09 / 16 / 80$ \\
\hline & u & 38. & 4. & PPB & $09 / 16 / 80$ \\
\hline & u & 36. & 4. & PPB & $09 / 16 / 80$ \\
\hline & U & 40.4 & 4. & PPM & $09 / 26 / 80$ \\
\hline & $u$ & 40.5 & 4. & PPM & $10 / 15 / 80$ \\
\hline & u & 41. & 4. & PPM & $11 / 19 / 80$ \\
\hline & u & 40.2 & 4. & PPM & $11 / 19 / 80$ \\
\hline & U & 41.3 & 4. & PPM & $11 / 19 / 80$ \\
\hline & U & 40.5 & 2. & PPM & $12 / 04 / 80$ \\
\hline & u & 44.8 & 2. & PPM & $12 / 04 / 80$ \\
\hline & u & 42. & 2. & PPM & $12 / 04 / 80$ \\
\hline & U & 40.8 & 2.5 & PPM & $12 / 05 / 80$ \\
\hline & $u$ & 42.5 & 2.5 & PPM & $12 / 05 / 80$ \\
\hline & u & 42.5 & & PPM & $12 / 05 / 80$ \\
\hline & $u$ & & 4. & PPM & $12 / 09 / 80$ \\
\hline & u & $41->$ & 4. & PPM & $12 / 15 / 80$ \\
\hline & u & & 4. & PPM & $12 / 15 / 80$ \\
\hline & U & 41. & 4. & PPM & $12 / 15 / 80$ \\
\hline & & & & & \\
\hline & & & .3 & $\%$ & $01 / 09 / 80$ \\
\hline & $C A$ & .97 & .3 & $\tilde{\psi}$ & $01 / 09 / 80$ \\
\hline
\end{tabular}


TABLE C-II (cont)

SAMPLE

NUMBER
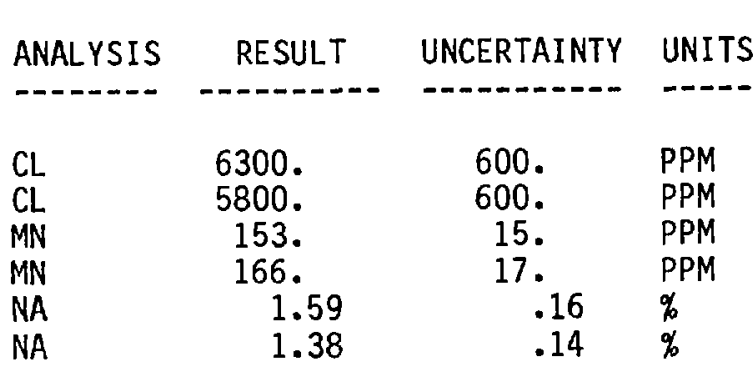

COMPLETION

80.00531

80.00532

$\begin{array}{lc}C A & 1.58 \\ C L & 700 . \\ C L & 620 . \\ C L & 790 . \\ C L & 700 . \\ C L & 700 . \\ \text { HG } & 170 . \\ \text { HG } & 165 . \\ \text { HG } & 160 . \\ K & 1.2 \\ K & 1.6 \\ K & 1.36 \\ \text { MN } & 89 . \\ \text { MN } & 90 . \\ \text { MN } & 96 . \\ N A & 92 . \\ U & 31.1 \\ U & 28.1\end{array}$

80.
90.

100.

80.

75.

20.

20.

20.

$\begin{array}{cl}.12 & \% \\ .16 & \% \\ .14 & \% \\ 9 . & \text { PPM } \\ 9 . & \text { PPM } \\ 10 . & \text { PPM } \\ 9 . & \text { PPM } \\ 3 . & \text { PPB } \\ 3 . & \text { PPB }\end{array}$

DATE

- D.-.-.-.

$01 / 09 / 80$

$01 / 09 / 80$

$01 / 09 / 80$

$01 / 09 / 80$

$01 / 09 / 80$

$01 / 09 / 80$

80.00533

$\begin{array}{ll}\text { B } & 35 . \\ \text { B } & 34 . \\ \text { B } & 32 . \\ \text { B } & 31 . \\ \text { B } & 33 . \\ \text { B } & 27 . \\ \text { B } & 33 .\end{array}$

$\begin{array}{lll}\text { 5. } & \text { PPM } & 12 / 05 / 80 \\ \text { 5. } & \text { PPM } & 12 / 05 / 80 \\ \text { 5. } & \text { PPM } & 12 / 05 / 80 \\ \text { 5. } & \text { PPM } & 12 / 05 / 80 \\ \text { 5. } & \text { PPM } & 12 / 05 / 80 \\ \text { 5. } & \text { PPM } & 12 / 05 / 80 \\ \text { 5. } & \text { PPM } & 12 / 23 / 80\end{array}$

80.00534

$\begin{array}{ll}\text { B } & 17 . \\ \text { B } & 17 . \\ \text { B } & 16 . \\ \text { B } & 16 . \\ \text { B } & 17 . \\ \text { B } & 18 .\end{array}$

5. PPM $12 / 05 / 80$

5. PPM $12 / 05 / 80$

5. PPM $12 / 05 / 80$

5. PPM $12 / 05 / 80$

5. PPM $12 / 05 / 80$

5. PPM $12 / 05 / 80$

80.00535

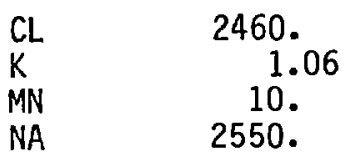

$\begin{array}{cll}250 . & \text { PPM } & 01 / 09 / 80 \\ .11 & \% & 01 / 09 / 80 \\ 1 . & \text { PPM } & 01 / 09 / 80 \\ 250 . & \text { PPM } & 01 / 09 / 80\end{array}$


TABLE C-II (cont)

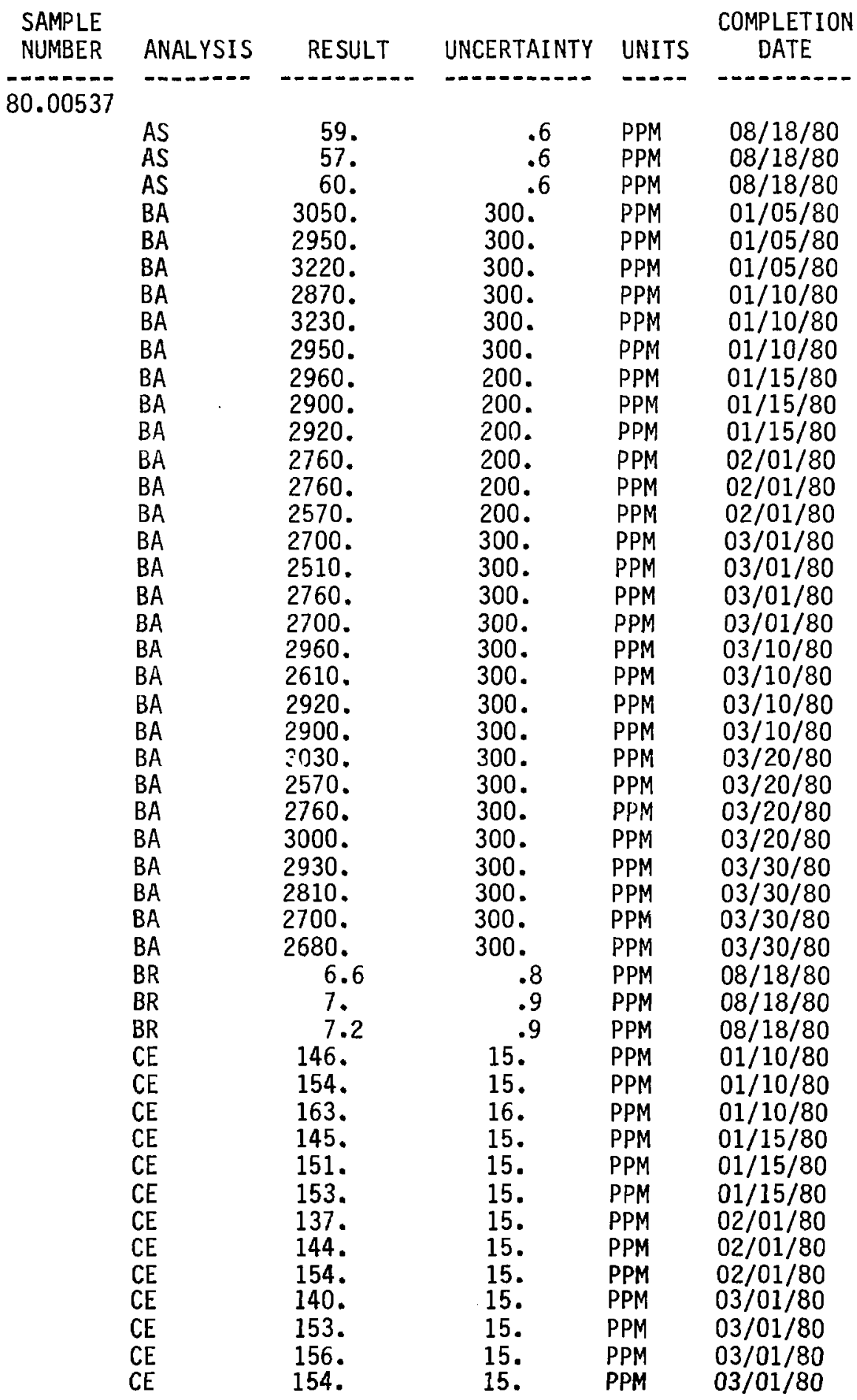


TABLE C-II (cont)

\begin{tabular}{|c|c|c|c|c|c|}
\hline $\begin{array}{l}\text { SAMPLE } \\
\text { NUMBER }\end{array}$ & ANALYSIS & RESULT & UNCERTAINTY & UNITS & $\begin{array}{l}\text { COMPLETION } \\
\text { DATE }\end{array}$ \\
\hline-7 & $-\cdots-. . .-$ & $\cdots$ & $-\cdots+-1$ & ---- & 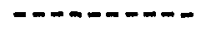 \\
\hline & CE & 153. & 15. & PPM & $03 / 10 / 80$ \\
\hline & $C E$ & 137. & 15. & PPM & $03 / 10 / 80$ \\
\hline & CE & 141. & 15. & PPM & $03 / 10 / 80$ \\
\hline & $\mathrm{CE}$ & 151. & 15. & PPM & $03 / 10 / 80$ \\
\hline & CE & 144. & 15. & PPM & $03 / 20 / 80$ \\
\hline & CE & 157. & 15. & PPM & $03 / 20 / 80$ \\
\hline & CE & 145. & 15. & PPM & $03 / 20 / 80$ \\
\hline & CE & 156. & 15. & PPM & $03 / 20 / 80$ \\
\hline & CE & 144. & 15. & PPM & $03 / 30 / 80$ \\
\hline & CE & 148. & 15. & PPM & $03 / 30 / 80$ \\
\hline & CE & 155. & 15. & PPM & $03 / 30 / 80$ \\
\hline & CE & 141. & 15. & PPM & $03 / 30 / 80$ \\
\hline & CO & 34. & 4. & PPM & $01 / 10 / 80$ \\
\hline & CO & 40. & 4. & PPM & $01 / 10 / 80$ \\
\hline & $\mathrm{CO}$ & 34. & 4. & PPM & $01 / 10 / 80$ \\
\hline & $\mathrm{CO}$ & 37. & 4. & PPM & $01 / 10 / 80$ \\
\hline & co & 38. & 4. & PPM & $01 / 10 / 80$ \\
\hline & $\mathrm{CO}$ & 37. & 4. & PPM & $01 / 10 / 80$ \\
\hline & $\mathrm{CO}$ & 42. & 4. & PPM & $01 / 15 / 80$ \\
\hline & CO & 43. & 4. & PPM & $01 / 15 / 80$ \\
\hline & $\mathrm{CO}$ & 38. & 4. & PPM & $01 / 15 / 80$ \\
\hline & CO & 40.6 & 4. & PPM & $02 / 01 / 80$ \\
\hline & CO & 42. & 4. & PPM & $02 / 01 / 80$ \\
\hline & CO & 41 . & 4. & PPM & $02 / 01 / 80$ \\
\hline & $\mathrm{CO}$ & 42. & 2. & PPM & $03 / 01 / 80$ \\
\hline & CO & 41.4 & 2. & PPM & $03 / 01 / 80$ \\
\hline & $\mathrm{CO}$ & 39.6 & 2. & PPM & 03/01/80 \\
\hline & CO & 41.3 & 2. & PPM & $03 / 01 / 80$ \\
\hline & & 40.7 & 2. & PPM & $03 / 10 / 80$ \\
\hline & $\begin{array}{l}\text { CO } \\
\text { CO }\end{array}$ & $\begin{array}{l}42 . \\
41 .\end{array}$ & $\begin{array}{l}2 . \\
2 .\end{array}$ & $\begin{array}{l}\text { PPM } \\
\text { PPM }\end{array}$ & $\begin{array}{l}03 / 10 / 80 \\
03 / 10 / 80\end{array}$ \\
\hline & CO & 43. & 2. & PPM & $03 / 10 / 80$ \\
\hline & $\mathrm{co}$ & 41.7 & 2. & PPM & $03 / 20 / 80$ \\
\hline & $\mathrm{CO}$ & 42.2 & 2. & PPM & $03 / 20 / 80$ \\
\hline & $\mathrm{CO}$ & 38. & 2. & PPM & $03 / 20 / 80$ \\
\hline & $\mathrm{CO}$ & 41. & 2. & PPM & $03 / 20 / 80$ \\
\hline & $\mathrm{CO}$ & 40.9 & 2. & PPM & $03 / 30 / 80$ \\
\hline & $\mathrm{CO}$ & 40.2 & 2. & PPM & $03 / 30 / 80$ \\
\hline & $\mathrm{CO}$ & 42.5 & 2. & PPM & $03 / 30 / 80$ \\
\hline & $\mathrm{CO}$ & 41.1 & 2. & PPM & $03 / 30 / 80$ \\
\hline & CR & 124. & 13. & PPM & $01 / 15 / 80$ \\
\hline & CR & 131. & 13. & PPM & $01 / 15 / 80$ \\
\hline & $C R$ & 136. & 13. & PPM & $02 / 01 / 80$ \\
\hline & CR & 129. & 13. & PPM & $02 / 01 / 80$ \\
\hline & CR & 122. & 13. & PPM & $02 / 01 / 80$ \\
\hline & CR & 126. & 8. & PPM & $03 / 01 / 80$ \\
\hline & CR & 125. & 8. & PPM & $03 / 01 / 80$ \\
\hline & CR & 136. & 8. & PPM & $03 / 01 / 80$ \\
\hline & CR & 130. & 8. & PPM & $03 / 01 / 80$ \\
\hline
\end{tabular}


TABLE C-II (cont)

\begin{tabular}{|c|c|c|c|c|c|}
\hline $\begin{array}{l}\text { SAMPLE } \\
\text { NUMBER }\end{array}$ & ANALYSIS & RESULT & UNCERTAINTY & UNITS & $\begin{array}{l}\text { COMPLETION } \\
\text { - DATE }\end{array}$ \\
\hline 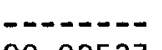 & $\cdots-\cdots-\infty$ & 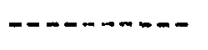 & $\cdots+\ldots-\ldots$ & $--\cdots$ & $---m-\cdots-n$ \\
\hline & $\mathrm{CR}$ & 128. & 8. & PPM & $03 / 10 / 80$ \\
\hline & $\mathrm{CR}$ & 123. & 8. & PPM & $03 / 10 / 80$ \\
\hline & $C R$ & 131. & 8. & PPM & $03 / 10 / 80$ \\
\hline & CR & 124. & 8. & PPM & $03 / 10 / 80$ \\
\hline & CR & 129. & 8. & PPM & $03 / 20 / 80$ \\
\hline & CR & 122 . & 8. & PPM & $03 / 20 / 80$ \\
\hline & CR & 129. & 8. & PPM & $03 / 20 / 80$ \\
\hline & $\mathrm{CR}$ & 121. & 8. & PPM & $03 / 20 / 80$ \\
\hline & $\mathrm{CR}$ & 132. & 8. & PPM & $03 / 30 / 80$ \\
\hline & CR & 136. & 8. & PPM & $03 / 30 / 80$ \\
\hline & $C R$ & 126. & 8. & PPM & $03 / 30 / 80$ \\
\hline & CS & 9. & .9 & PPM & $01 / 10 / 80$ \\
\hline & CS & 8.5 & .9 & PPM & $01 / 10 / 80$ \\
\hline & CS & 8.6 & .9 & $P P M$ & $01 / 10 / 80$ \\
\hline & CS & 10.2 & 1. & PPM & $01 / 15 / 80$ \\
\hline & $\mathrm{CS}$ & 10.2 & 1. & PPM & $01 / 15 / 80$ \\
\hline & CS & 8.8 & 1. & PPM & $01 / 15 / 80$ \\
\hline & CS & 9.3 & 1. & PPM & $02 / 01 / 80$ \\
\hline & $\mathrm{CS}$ & 9.7 & 1. & PPM & $02 / 01 / 80$ \\
\hline & CS & 9.3 & 1 . & PPM & $02 / 01 / 80$ \\
\hline & CS & 7.79 & .8 & PPM & $03 / 01 / 80$ \\
\hline & CS & 8.77 & .8 & PPM & $03 / 01 / 80$ \\
\hline & CS & 8.11 & .8 & PPM & $03 / 01 / 80$ \\
\hline & $\mathrm{CS}$ & 8.4 & .8 & PPM & $03 / 01 / 80$ \\
\hline & CS & 8.5 & .8 & PPM & $03 / 10 / 80$ \\
\hline & $\mathrm{CS}$ & 8.77 & .8 & PPM & $03 / 10 / 80$ \\
\hline & CS & 8.34 & .8 & PPM & $03 / 10 / 80$ \\
\hline & CS & 8. & .8 & PPM & $03 / 10 / 80$ \\
\hline & CS & 7.57 & .8 & PPM & $03 / 20 / 80$ \\
\hline & CS & 8.39 & .8 & PPM & $03 / 20 / 80$ \\
\hline & CS & 8.94 & .8 & PPM & $03 / 20 / 80$ \\
\hline & CS & 8. & .8 & PPM & $03 / 20 / 80$ \\
\hline & CS & 8.86 & .8 & PPM & $03 / 30 / 80$ \\
\hline & $\mathrm{CS}$ & 7.94 & .8 & PPM & $03 / 30 / 80$ \\
\hline & CS & 8.06 & .8 & PPM & $03 / 30 / 80$ \\
\hline & CS & 8.14 & .8 & PPM & $03 / 30 / 80$ \\
\hline & CS & 9.1 & 1 . & PPM & $11 / 18 / 80$ \\
\hline & DY & 10.2 & 1.5 & PPM & $10 / 23 / 80$ \\
\hline & $E U$ & 2.58 & .2 & PPM & $01 / 15 / 80$ \\
\hline & EU & 2.6 & .2 & PPM & $01 / 15 / 80$ \\
\hline & EU & 2.43 & .2 & PPM & $01 / 15 / 80$ \\
\hline & EU & 2.26 & .2 & PPM & $02 / 01 / 80$ \\
\hline & EU & 2.51 & .2 & PPM & $02 / 01 / 80$ \\
\hline & EU & 2.83 & .2 & PPM & $02 / 01 / 80$ \\
\hline & EU & 2.46 & .15 & PPM & $03 / 01 / 80$ \\
\hline & EU & 2.5 & .15 & PPM & $03 / 01 / 80$ \\
\hline & EU & 2.43 & .15 & PPM & $03 / 01 / 80$ \\
\hline & EU & 2.51 & .15 & PPM & $03 / 01 / 80$ \\
\hline & EU & 2.58 & .15 & PPM & $03 / 10 / 80$ \\
\hline
\end{tabular}


TABLE C-II (cont)

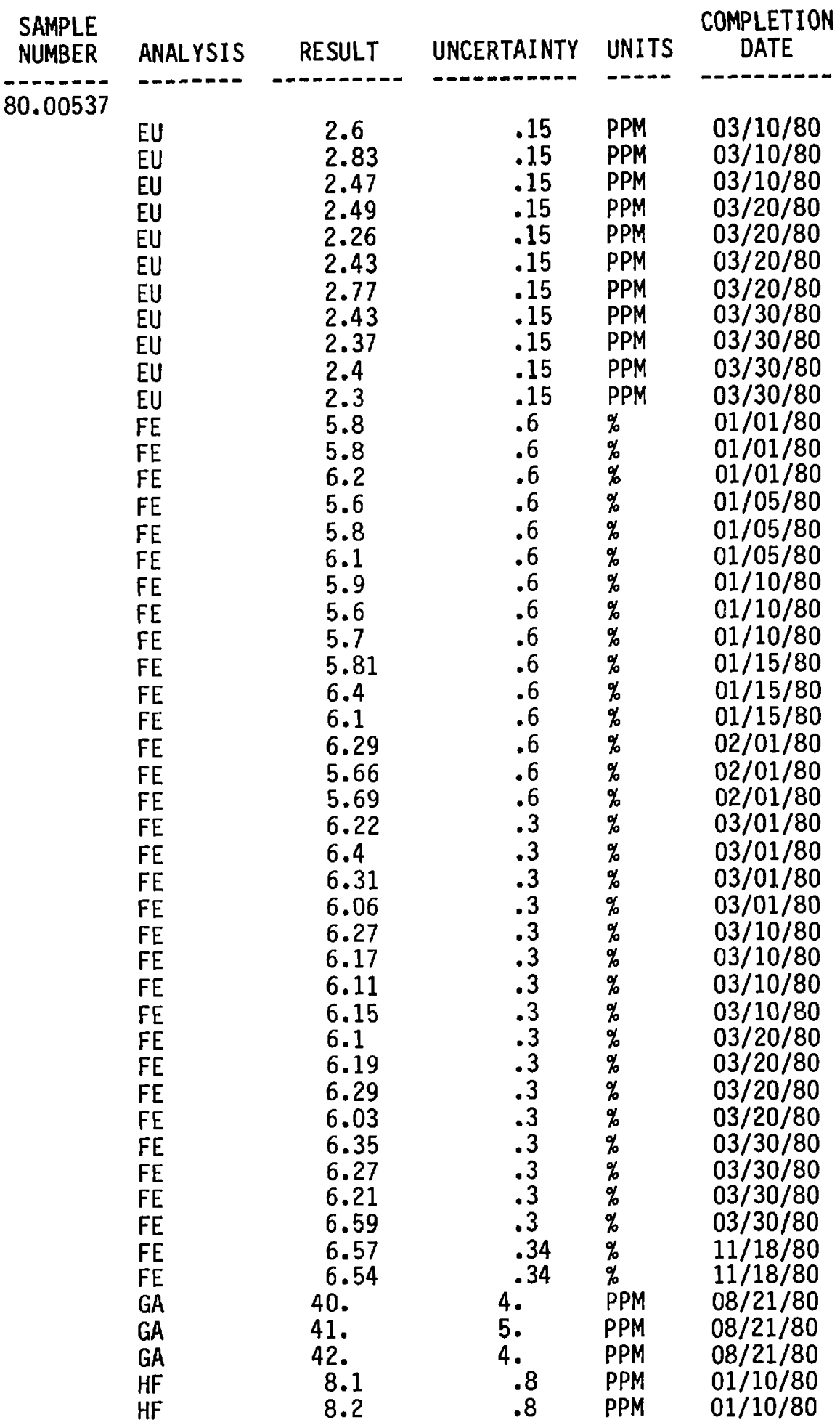


TABLE C-II (cont)

\begin{tabular}{|c|c|c|c|c|c|}
\hline $\begin{array}{l}\text { SAMPLE } \\
\text { NUMBER }\end{array}$ & ANALYSIS & RESULT & UNCERTAINTY & UNITS & $\begin{array}{l}\text { COMPLETION } \\
\text { DATE }\end{array}$ \\
\hline .00537 & 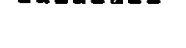 & $\cdots-\cdots$ & & $\cdots$ & -- \\
\hline & HF & 8.1 & .8 & PPM & $01 / 10 / 80$ \\
\hline & HF & 8.1 & .8 & PPM & $01 / 15 / 80$ \\
\hline & $\mathrm{HF}$ & 7.9 & .8 & PPM & $01 / 15 / 80$ \\
\hline & $\mathrm{HF}$ & 8.7 & .8 & PPM & $01 / 15 / 80$ \\
\hline & $H F$ & 7.3 & .7 & PPM & $02 / 01 / 80$ \\
\hline & $H F$ & 7.3 & .7 & PPM & $02 / 01 / 80$ \\
\hline & $\mathrm{HF}$ & 8.3 & .8 & PPM & $02 / 01 / 80$ \\
\hline & $\mathrm{HF}$ & 8.25 & .8 & PPM & $03 / 01 / 80$ \\
\hline & $\mathrm{HF}$ & 8.3 & .8 & PPM & $03 / 01 / 80$ \\
\hline & $\mathrm{HF}$ & 7.41 & .8 & PPM & $03 / 01 / 80$ \\
\hline & HF & 8.09 & .8 & PPM & $03 / 01 / 80$ \\
\hline & $\mathrm{HF}$ & 8.7 & .8 & PPM & $03 / 10 / 80$ \\
\hline & $\mathrm{HF}$ & 7.97 & .8 & PPM & $03 / 10 / 80$ \\
\hline & HF & 8.1 & .8 & PPM & $03 / 10 / 80$ \\
\hline & HF & 7.9 & .8 & PPM & $03 / 10 / 80$ \\
\hline & HF & 7.83 & .8 & PPM & $03 / 20 / 80$ \\
\hline & HF & 8.26 & .8 & PPM & $03 / 20 / 80$ \\
\hline & HF & 7.3 & .8 & PPM & $03 / 20 / 80$ \\
\hline & HF & 7.3 & .8 & PPM & $03 / 20 / 80$ \\
\hline & $\mathrm{HF}$ & 7.71 & .8 & PPM & $03 / 30 / 80$ \\
\hline & $\mathrm{HF}$ & 7.62 & .8 & PPM & $03 / 30 / 80$ \\
\hline & $\mathrm{HF}$ & 8.11 & .8 & PPM & $03 / 30 / 80$ \\
\hline & HF & 8.35 & .8 & PPM & $03 / 30 / 80$ \\
\hline & $k$ & 1.97 & .04 & $\%$ & $09 / 22 / 80$ \\
\hline & ND & 59.5 & 6. & PPM & $01 / 10 / 80$ \\
\hline & ND & 60.7 & 6. & PPM & $01 / 10 / 80$ \\
\hline & NI & 87. & 8. & PPM & $01 / 10 / 80$ \\
\hline & NI & 83. & 8. & PPM & $01 / 10 / 80$ \\
\hline & NI & 83. & 8. & PPM & $01 / 10 / 80$ \\
\hline & $\mathrm{RB}$ & 124. & 12. & PPM & $01 / 10 / 80$ \\
\hline & RB & 116. & 11. & PPM & $01 / 10 / 80$ \\
\hline & $\mathrm{RB}$ & 112. & 11 . & PPM & $01 / 10 / 80$ \\
\hline & RB & 118. & 10. & PPM & $01 / 15 / 80$ \\
\hline & $\mathrm{RB}$ & 112. & 10. & PPM & $01 / 15 / 80$ \\
\hline & $\mathrm{RB}$ & 119. & 10. & PPM & $01 / 15 / 80$ \\
\hline & RB & 125. & 10. & PPM & $02 / 01 / 80$ \\
\hline & RB & 115. & 10 & PPM & $02 / 01 / 80$ \\
\hline & RB & 111. & 10. & PPM & $02 / 01 / 80$ \\
\hline & $\mathrm{RB}$ & 119. & 10. & PPM & $03 / 01 / 80$ \\
\hline & RB & 117. & 10. & PPM & $03 / 01 / 80$ \\
\hline & RB & 112 . & 10. & PPM & $03 / 01 / 80$ \\
\hline & RB & 118. & 10. & PPM & $03 / 01 / 80$ \\
\hline & $\begin{array}{l}\mathrm{RB} \\
\mathrm{RB}\end{array}$ & 125. & 10. & PPM & $03 / 10 / 80$ \\
\hline & $\begin{array}{l}R B \\
R B\end{array}$ & $\begin{array}{l}112 . \\
122 .\end{array}$ & $\begin{array}{l}10 . \\
10 .\end{array}$ & $\begin{array}{l}\text { PPM } \\
\text { PPM }\end{array}$ & $\begin{array}{l}03 / 10 / 80 \\
03 / 10 / 80\end{array}$ \\
\hline & $\mathrm{RB}$ & 115. & 10. & PPM & $03 / 10 / 80$ \\
\hline & $\mathrm{RB}$ & 122. & 10. & PPM & $03 / 20 / 80$ \\
\hline & RB & 135. & 10. & PPM & $03 / 20 / 80$ \\
\hline & RB & 119. & 10. & PPM & $03 / 20 / 80$ \\
\hline
\end{tabular}


TABLE C-II (cont)

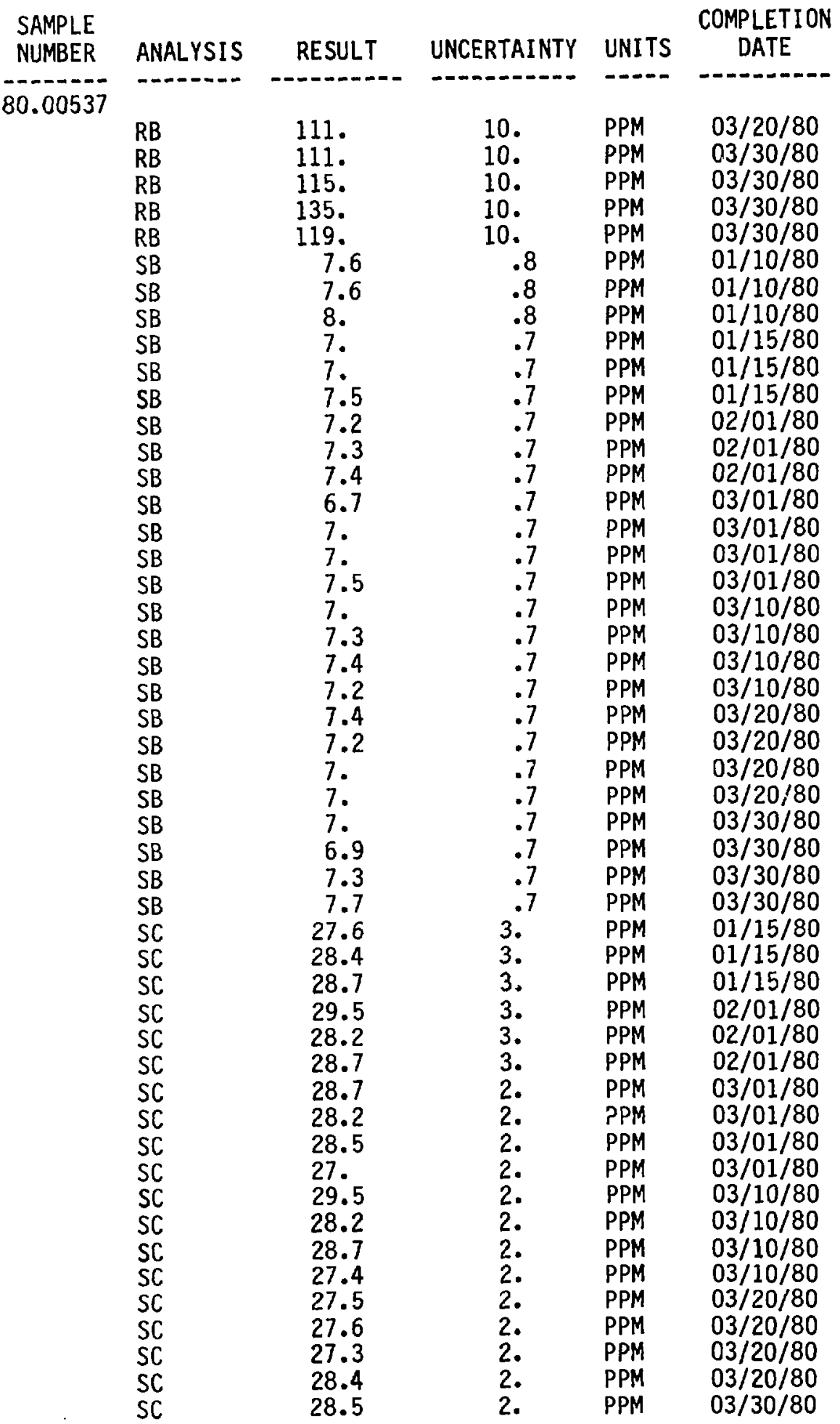


TABLE C-II (cont)

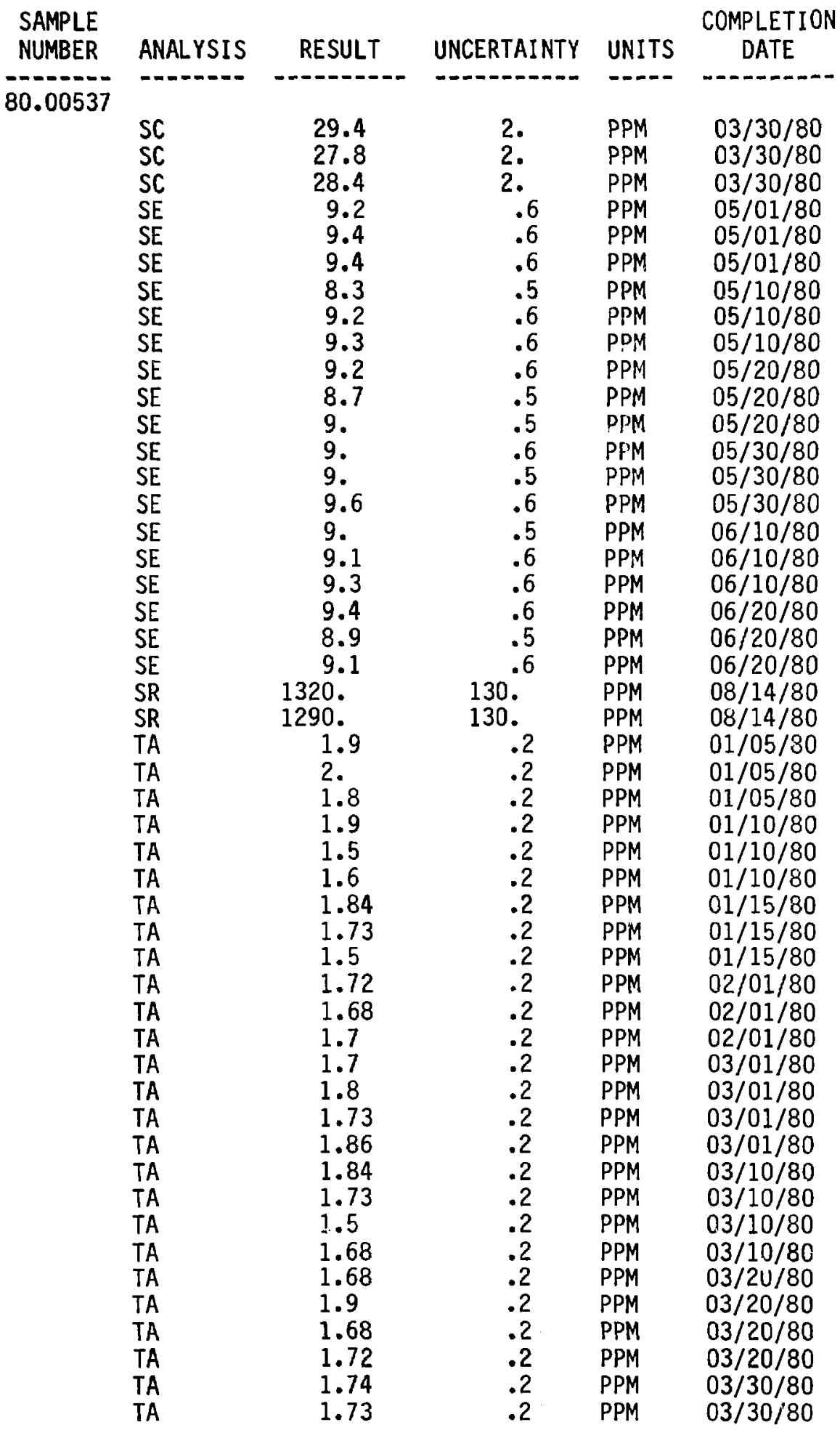


TABLE C-II (cont)

\begin{tabular}{|c|c|c|c|c|c|}
\hline $\begin{array}{l}\text { SAMPLE } \\
\text { NUMBER }\end{array}$ & ANALYSIS & RESULT & UNCERTAINTY & UNITS & $\begin{array}{c}\text { COMPLETION } \\
\text { DATE }\end{array}$ \\
\hline & $-m-\cdots$ & 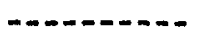 & - - & $---\infty$ & $---n--\infty-n-$ \\
\hline 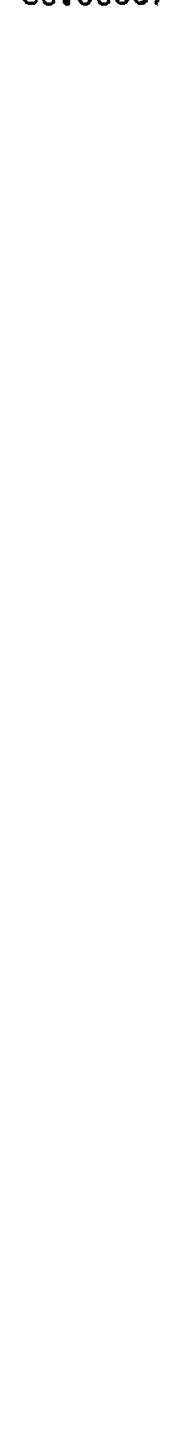 & $\begin{array}{l}\text { TA } \\
\text { TA } \\
T B \\
T B \\
T B \\
T H \\
T H \\
T H \\
T H \\
T H \\
T H \\
T H \\
T H \\
T H \\
T H \\
T H \\
T H \\
T H \\
T H \\
T H \\
T H \\
T H \\
T H \\
T H \\
T H \\
T H \\
T H \\
T H \\
T H \\
T H \\
W \\
W \\
W \\
Z N \\
Z N \\
Z N \\
Z R \\
Z R \\
Z R\end{array}$ & $\begin{array}{c}1.66 \\
1.82 \\
2 . \\
1.9 \\
2.1 \\
25.4 \\
24.2 \\
23.5 \\
25.6 \\
25.8 \\
25.4 \\
25.2 \\
25.4 \\
23.2 \\
25.4 \\
24.2 \\
25.3 \\
26.1 \\
23.2 \\
25.4 \\
23.7 \\
25.2 \\
25.9 \\
26.3 \\
25.8 \\
25.6 \\
25.7 \\
24.3 \\
24.3 \\
24.9 \\
6 . \\
5.9 \\
5.5 \\
213 . \\
210 . \\
240 . \\
281 . \\
295 . \\
281 .\end{array}$ & $\begin{array}{l}.2 \\
.2 \\
.2 \\
.2 \\
.2 \\
2 . \\
2 . \\
2 . \\
2 . \\
2 . \\
2 . \\
2 . \\
2 . \\
2 . \\
1 . \\
1 . \\
1 . \\
1 . \\
1 . \\
1 . \\
1 . \\
1 . \\
1 . \\
1 . \\
1 . \\
1 . \\
1 . \\
1 . \\
1 . \\
1 . \\
1.4 \\
1.4 \\
1.3 \\
20 . \\
20 . \\
20 . \\
40 . \\
40 . \\
40 .\end{array}$ & 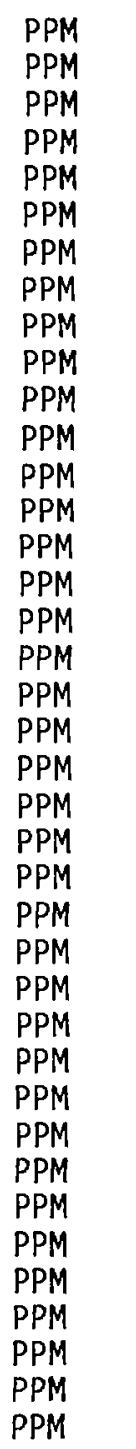 & $\begin{array}{l}03 / 30 / 80 \\
03 / 30 / 80 \\
01 / 10 / 80 \\
01 / 10 / 80 \\
01 / 10 / 80 \\
01 / 10 / 80 \\
01 / 10 / 80 \\
01 / 10 / 80 \\
01 / 15 / 80 \\
01 / 15 / 80 \\
01 / 15 / 80 \\
02 / 01 / 80 \\
02 / 01 / 80 \\
02 / 01 / 80 \\
03 / 01 / 80 \\
03 / 01 / 80 \\
03 / 01 / 80 \\
03 / 01 / 80 \\
03 / 10 / 80 \\
03 / 10 / 80 \\
03 / 10 / 80 \\
03 / 10 / 80 \\
03 / 20 / 80 \\
03 / 20 / 80 \\
03 / 20 / 80 \\
03 / 20 / 80 \\
03 / 30 / 80 \\
03 / 30 / 80 \\
03 / 30 / 80 \\
03 / 30 / 80 \\
08 / 18 / 80 \\
08 / 18 / 80 \\
08 / 18 / 80 \\
01 / 10 / 80 \\
01 / 10 / 80 \\
01 / 10 / 80 \\
01 / 10 / 80 \\
01 / 10 / 80 \\
01 / 10 / 80\end{array}$ \\
\hline 80.00540 & $\begin{array}{l}H G \\
H G \\
H G \\
H G\end{array}$ & $\begin{array}{l}1.1 \\
1 . \\
1.1 \\
1.1\end{array}$ & $\begin{array}{l}.1 \\
.1 \\
.1 \\
.1\end{array}$ & $\begin{array}{l}\text { PPB } \\
\text { PPB } \\
\text { PPB } \\
\text { PPB }\end{array}$ & $\begin{array}{l}04 / 04 / 80 \\
04 / 04 / 80 \\
04 / 04 / 80 \\
04 / 14 / 80\end{array}$ \\
\hline 80.00570 & $\begin{array}{l}A L \\
A L\end{array}$ & $\begin{array}{l}8.95 \\
8.92\end{array}$ & .46 & $\begin{array}{l}\% \\
\% \\
\%\end{array}$ & $\begin{array}{l}07 / 08 / 80 \\
09 / 08 / 80\end{array}$ \\
\hline
\end{tabular}


TABLE C-II (cont)

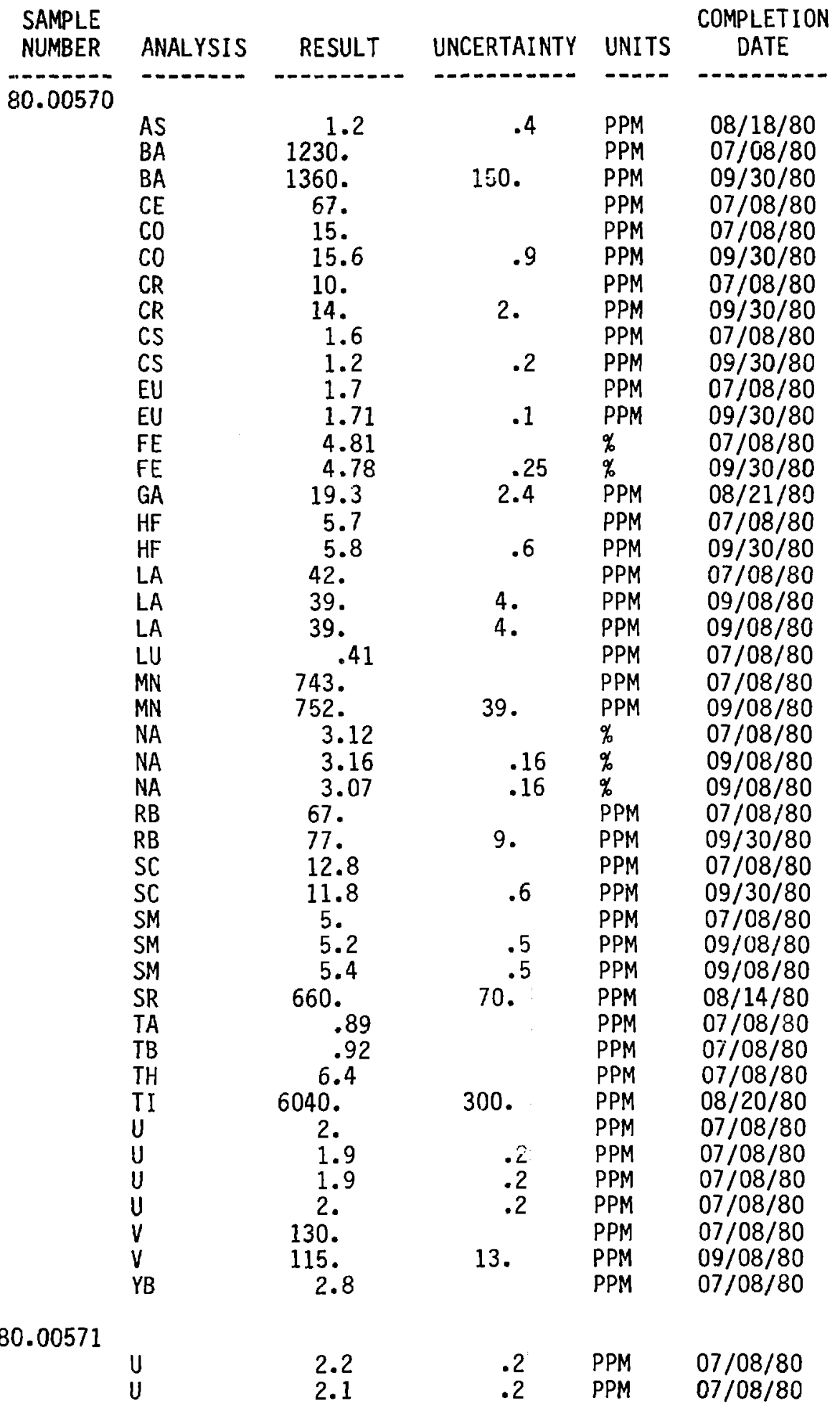


TABLE C-II (cont)

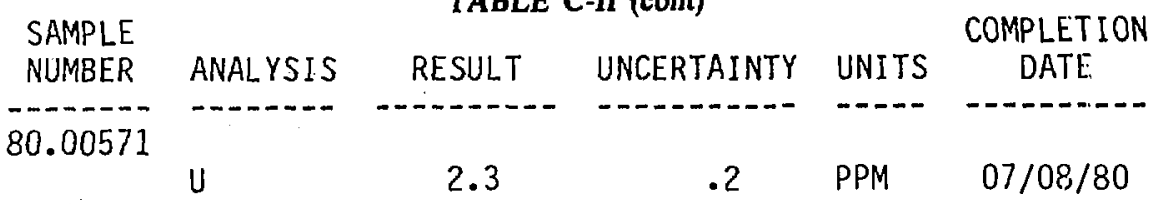

80.00572

\begin{tabular}{|c|c|c|c|c|}
\hline$A L$ & 7.18 & & $\%$ & $07 / 08 / 80$ \\
\hline$A L$ & 7.2 & .37 & $\%$ & $09 / 08 / 80$ \\
\hline AS & 500. & 200. & PPB & $08 / 18 / 80$ \\
\hline$B A$ & 970. & & PPM & $07 / 08 / 80$ \\
\hline$B A$ & 740. & 100. & PPM & $09 / 30 / 80$ \\
\hline CE & 45. & & PPM & $07 / 08 / 80$ \\
\hline $\mathrm{CO}$ & 36. & & PPM & $07 / 08 / 80$ \\
\hline $\mathrm{CO}$ & 37. & 2. & PPM & $09 / 30 / 80$ \\
\hline CR & 19. & & PPM & $07 / 08 / 80$ \\
\hline$C R$ & 15. & 3. & PPM & $09 / 30 / 80$ \\
\hline CS & 1.6 & & PPM & $07 / 08 / 80$ \\
\hline CS & 1.3 & .4 & PPM & $09 / 30 / 80$ \\
\hline$E U$ & 2.2 & & PPM & $07 / 08 / 80$ \\
\hline EU & 1.97 & .13 & PPM & $09 / 30 / 80$ \\
\hline$F E$ & 9.6 & & $\%$ & $07 / 08 / 80$ \\
\hline $\mathrm{FE}$ & 9.62 & .49 & $\%$ & $09 / 30 / 80$ \\
\hline$G A$ & 20.6 & 2.6 & PPM & $08 / 21 / 80$ \\
\hline HF & 6.3 & & PPM & $07 / 08 / 80$ \\
\hline$H F$ & 5.5 & .6 & PPM & $09 / 30 / 80$ \\
\hline LA & 24. & & PPM & $07 / 08 / 80$ \\
\hline LA & 27. & 3. & PPM & $09 / 08 / 80$ \\
\hline LA & 27. & 3. & PPM & $09 / 08 / 80$ \\
\hline$L U$ & 1. & & PPM & $07 / 08 / 80$ \\
\hline MN & 1400 . & & PPM & $07 / 08 / 80$ \\
\hline$M N$ & 1405 & 72. & PPM & $09 / 08 / 80$ \\
\hline NA & 2.29 & & $\%$ & $07 / 08 / 80$ \\
\hline NA & 2.54 & .13 & $\%$ & $09 / 08 / 80$ \\
\hline$N A$ & 2.51 & .13 & $\%$ & $09 / 08 / 80$ \\
\hline RB & 54. & & PPM & $07 / 08 / 80$ \\
\hline$R B$ & 44. & 7. & PPM & $09 / 30 / 80$ \\
\hline SC & 35.3 & & PPM & $07 / 08 / 80$ \\
\hline sic & 32.5 & 1.6 & PPM & $09 / 30 / 80$ \\
\hline SM & 5.4 & & PPM & $07 / 08 / 80$ \\
\hline SM & 6.2 & .6 & PPM & $09 / 08 / 80$ \\
\hline SM & 6.1 & .6 & PPM & $09 / 08 / 80$ \\
\hline$S R$ & 330. & 40. & PPM & $08 / 14 / 80$ \\
\hline $\mathrm{TA}$ & 1.14 & & PPM & $07 / 08 / 80$ \\
\hline TB & 1.2 & & PPM & $07 / 08 / 80$ \\
\hline TH & 6.1 & & PPM & $07 / 08 / 80$ \\
\hline TI & 1.27 & .06 & $\%$ & $08 / 20 / 80$ \\
\hline$U$ & 1.7 & .2 & PPM & $07 / 08 / 80$ \\
\hline$U$ & 1.8 & .2 & PPM & $07 / 08 / 80$ \\
\hline$U$ & 1.8 & & PPM & $07 / 08 / 80$ \\
\hline V & 395. & & PPM & $07 / 08 / 80$ \\
\hline $\begin{array}{l}V \\
V R\end{array}$ & 403. & 42. & PPM & 09/08/80 \\
\hline & 4.3 & & PPM & $07 / 08 / 80$ \\
\hline
\end{tabular}


TABLE C-II (cont)

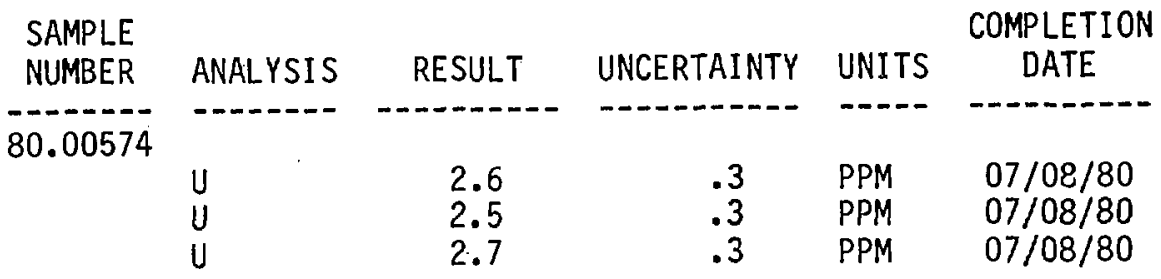

80.00575

\begin{tabular}{|c|c|c|c|c|}
\hline $\mathrm{AL}$ & 3.4 & .34 & $\%$ & $07 / 28 / 80$ \\
\hline AS & 339. & 34. & PPM & $05 / 19 / 80$ \\
\hline AS & 405. & 41. & PPM & $05 / 19 / 80$ \\
\hline AS & 461. & 40. & PPM & $07 / 14 / 80$ \\
\hline AS & 476. & 20. & PPM & $07 / 14 / 80$ \\
\hline AS & 480. & 20. & PPM & $07 / 14 / 80$ \\
\hline$C D$ & 3.7 & .4 & PPM & $05 / 19 / 80$ \\
\hline$C D$ & 3.8 & .4 & PPM & $05 / 19 / 80$ \\
\hline $\mathrm{CO}$ & 9.3 & 1.3 & PPM & $07 / 18 / 80$ \\
\hline $\mathrm{CO}$ & 10.8 & 1.1 & PPM & $07 / 28 / 80$ \\
\hline $\mathrm{CO}$ & 9.9 & 1. & PPM & $07 / 28 / 80$ \\
\hline CR & 16. & 6. & PPM & $07 / 28 / 80$ \\
\hline FE & 26.3 & 2.7 & $\%$ & $07 / 18 / 80$ \\
\hline FE & 26.4 & 3. & $\%$ & $07 / 28 / 80$ \\
\hline $\mathrm{FE}$ & 26.2 & 3. & $\%$ & $07 / 28 / 80$ \\
\hline$K$ & 590. & 100 & PPM & $09 / 23 / 80$ \\
\hline K & 660. & 100 & PPM & $09 / 23 / 80$ \\
\hline$K$ & 650. & 100 & PPM & $09 / 23 / 80$ \\
\hline$K$ & 510. & 100. & PPM & $09 / 23 / 80$ \\
\hline LA & 8.5 & 2.5 & PPM & $09 / 23 / 80$ \\
\hline LA & 8.4 & 2.5 & PPM & $09 / 23 / 80$ \\
\hline LA & 8.3 & 2.5 & PPM & $09 / 23 / 80$ \\
\hline LA & 8.7 & 2.5 & PPM & $09 / 23 / 80$ \\
\hline LI & 8. & 2. & PPM & $05 / 19 / 80$ \\
\hline LI & 11. & 2. & PPM & $05 / 19 / 80$ \\
\hline$N A$ & 600 & 100 & PPM & $09 / 23 / 80$ \\
\hline NA & 620 & 100 & PPM & $09 / 23 / 80$ \\
\hline NA & 600 & 100 & PPM & $09 / 23 / 80$ \\
\hline NA & 615. & 100. & PPM & $09 / 23 / 80$ \\
\hline SE & 12. & 6. & PPM & $08 / 20 / 80$ \\
\hline U & 36.6 & 4. & PPM & $04 / 01 / 80$ \\
\hline U & 36.5 & 4. & PPM & $04 / 01 / 80$ \\
\hline U & 38.3 & 4. & PPM & $04 / 01 / 80$ \\
\hline U & 37. & 4. & PPM & $04 / 10 / 80$ \\
\hline U & 35.6 & 4. & PPM & $04 / 10 / 80$ \\
\hline u & 41.6 & 4. & PPM & $04 / 10 / 80$ \\
\hline U & 39.8 & 4. & PPM & $04 / 18 / 80$ \\
\hline U & 42.5 & 4. & PPM & $04 / 20 / 80$ \\
\hline U & 38.7 & 4. & PPM & $04 / 20 / 80$ \\
\hline U & 40.7 & 4. & PPM & $04 / 20 / 80$ \\
\hline U & 39. & 3. & PPM & $05 / 29 / 80$ \\
\hline U & 30. & 3. & PPM & $05 / 29 / 80$ \\
\hline
\end{tabular}


TABLE C-II (cont)

\begin{tabular}{clcccc}
$\begin{array}{c}\text { SAMPLE } \\
\text { NUMBER }\end{array}$ & ANALYSIS & RESULT & UNCERTAINTY & UNITS & $\begin{array}{c}\text { COMPLETION } \\
\text { DATE }\end{array}$ \\
\hline 80.00575 & & & & & \\
& $U$ & 34. & 3. & $P P M$ & $05 / 29 / 80$ \\
& $U$ & 31. & 3. & PPM & $05 / 29 / 80$ \\
& $U$ & 37. & 3. & $P P M$ & $05 / 29 / 80$ \\
& $U$ & 33.5 & 3. & PPM & $05 / 29 / 80$ \\
& $U$ & 33.5 & 3. & PPM & $07 / 08 / 80$ \\
& $U$ & 33.7 & 3. & PPM & $07 / 08 / 80$ \\
& $U$ & 44.6 & 10. & PPM & $07 / 14 / 80$ \\
& $U$ & 33. & 4. & PPM & $07 / 14 / 80$ \\
& $U$ & 40.7 & 10. & $P P M$ & $07 / 14 / 80$ \\
& $U$ & 82. & 10. & $P P M$ & $07 / 28 / 80$ \\
& $V$ & 785. & 80. & $P P M$ & $07 / 28 / 80$
\end{tabular}

80.00577

\begin{tabular}{|c|c|c|c|c|}
\hline$A G$ & 18.3 & 2. & PPM & $01 / 02 / 80$ \\
\hline$A G$ & 18.1 & 2. & PPM & $01 / 02 / 80$ \\
\hline$A G$ & 17.1 & 2. & PPM & $01 / 02 / 80$ \\
\hline$A G$ & 17.2 & 1.9 & PPM & $01 / 10 / 80$ \\
\hline$A G$ & 17.3 & 1.9 & PPM & $01 / 10 / 80$ \\
\hline$A G$ & 17.1 & 1.8 & PPM & $01 / 10 / 80$ \\
\hline$A G$ & 18.8 & 2. & PPM & $01 / 15 / 80$ \\
\hline$A G$ & 18.6 & 2. & PPM & $01 / 15 / 80$ \\
\hline$A G$ & 17.2 & 1.8 & PPM & $01 / 15 / 80$ \\
\hline$A G$ & 18.3 & 1.9 & PPM & $01 / 20 / 80$ \\
\hline$\overline{A G}$ & 15.8 & 1.7 & PPM & $01 / 20 / 80$ \\
\hline$A G$ & 17.5 & 1.9 & PPM & $01 / 20 / 80$ \\
\hline$A L$ & 18.7 & 1.9 & $\%$ & $10 / 23 / 80$ \\
\hline $\mathrm{AL}$ & 18.3 & 1.8 & $\%$ & $10 / 28 / 80$ \\
\hline AS & 42. & 4. & PPM & $05 / 19 / 80$ \\
\hline AS & 32. & 3. & PPM & $05 / 19 / 80$ \\
\hline $\mathrm{CD}$ & 4.4 & .4 & PPM & $05 / 19 / 80$ \\
\hline $\mathrm{CD}$ & 4.9 & & PPM & $05 / 19 / 80$ \\
\hline $\mathrm{CE}$ & 49. & 5. & PPM & $11 / 18 / 80$ \\
\hline $\mathrm{CE}$ & 52. & 5. & PPM & $11 / 18 / 80$ \\
\hline$C R$ & 33. & 4. & PPM & $11 / 18 / 80$ \\
\hline$C R$ & 35. & 4. & PPM & $11 / 18 / 80$ \\
\hline CS & 5.1 & .6 & PPM & $11 / 18 / 80$ \\
\hline $\mathrm{CS}$ & 5.6 & .6 & PPM & $11 / 18 / 80$ \\
\hline DY & 2.8 & .4 & PPM & $10 / 23 / 80$ \\
\hline DY & 3.2 & .6 & PPM & $10 / 28 / 80$ \\
\hline EU & 740 & 110. & PPB & $11 / 18 / 80$ \\
\hline EU & 740. & 110. & PPB & $11 / 18 / 80$ \\
\hline $\mathrm{FE}$ & 1.82 & .1 & $\%$ & $11 / 18 / 80$ \\
\hline $\mathrm{FE}$ & 1.83 & .1 & $\%$ & $11 / 18 / 80$ \\
\hline HF & 8.6 & .9 & PPM & $11 / 18 / 80$ \\
\hline $\mathrm{HF}$ & 8.3 & .9 & PPM & $11 / 18 / 80$ \\
\hline LA & 28. & 3. & PPM & $11 / 18 / 80$ \\
\hline LI & 35. & 2. & PPM & $05 / 19 / 80$ \\
\hline LI & 42. & 2. & PPM & $05 / 19 / 80$ \\
\hline
\end{tabular}


TABLE C-II (cont)

\begin{tabular}{|c|c|c|c|c|c|}
\hline \multirow{2}{*}{$\begin{array}{l}\text { SAMPLE } \\
\text { NUMBER } \\
80.00577\end{array}$} & ANALYSIS & RESULT & UNCERTAINTY & UNITS & $\begin{array}{c}\text { COMPLETION } \\
\text { DATE }\end{array}$ \\
\hline & $\ldots \ldots$ & $--n-\infty-n$ & - - & $-\cdots-n$ & $---\infty-\infty$ \\
\hline & MN & 980. & 50. & PPM & $10 / 23 / 80$ \\
\hline & $\mathrm{MN}$ & 930. & 100. & PPM & $10 / 28 / 80$ \\
\hline & NA & 5240. & 500. & $P P M$ & $10 / 28 / 80$ \\
\hline & NA & 5770. & 300. & PPM & $11 / 18 / 80$ \\
\hline & $R B$ & 65 & 8. & PPM & $11 / 18 / 80$ \\
\hline & RB & 69. & 8. & PPM & $11 / 18 / 80$ \\
\hline & SM & 3.5 & .4 & PPM & $11 / 18 / 80$ \\
\hline & $\mathrm{TH}$ & 8.6 & .9 & PPM & $06 / 06 / 80$ \\
\hline & $\mathrm{TH}$ & 8.9 & .9 & PPM & $06 / 06 / 80$ \\
\hline & $\mathrm{TH}$ & 8.9 & .9 & PPM & $06 / 06 / 80$ \\
\hline & TH & 8.1 & .8 & PPM & $11 / 18 / 80$ \\
\hline & TH & 8.4 & .8 & PPM & $11 / 18 / 80$ \\
\hline & U & 2.9 & .3 & PPM & $07 / 08 / 80$ \\
\hline & U & 3. & .3 & PPM & $07 / 08 / 80$ \\
\hline & U & 3. & .3 & PPM & $07 / 08 / 80$ \\
\hline & V & 65. & 10. & PPM & $10 / 28 / 80$ \\
\hline & $W$ & 1.4 & .2 & PPM & $10 / 20 / 80$ \\
\hline & W & 1.4 & .2 & PPM & $10 / 20 / 80$ \\
\hline & YB & 1.8 & .4 & PPM & $11 / 18 / 80$ \\
\hline & YB & 1.8 & .3 & PPM & $11 / 18 / 80$ \\
\hline
\end{tabular}

80.00579

$\begin{array}{lr}\text { AS } & 4710 . \\ \text { AS } & 3870 . \\ \text { CD } & .59 \\ \text { CD } & .47 \\ \text { HG } & 350 . \\ \text { HG } & 320 . \\ \text { HG } & 320 . \\ \text { HG } & 400 . \\ \text { HG } & 330 . \\ \text { LI } & 125 . \\ \text { LI } & 120 .\end{array}$

$\begin{array}{ccc}\text { 471. } & \text { PPM } & 05 / 19 / 80 \\ 390 . & \text { PPM } & 05 / 19 / 80 \\ .05 & \text { PPB } & 05 / 19 / 80 \\ .05 & \text { PPB } & 05 / 19 / 80 \\ 70 . & \text { PPB } & 05 / 02 / 80 \\ 70 . & \text { PPB } & 05 / 02 / 80 \\ 70 . & \text { PPB } & 05 / 02 / 80 \\ 70 . & \text { PPB } & 05 / 02 / 80 \\ 70 . & \text { PPB } & 05 / 02 / 80 \\ 5 . & \text { PPM } & 05 / 19 / 80 \\ 6 . & \text { PPM } & 05 / 19 / 80\end{array}$

80.00581

$\begin{array}{lcccc}\text { AS } & 104 . & 10 . & \text { PPM } & 05 / 19 / 80 \\ \text { AS } & 110 . & 11 . & \text { PPM } & 05 / 19 / 80 \\ \text { CD } & 630 . & 50 . & \text { PPB } & 05 / 19 / 80 \\ \text { CD } & 710 . & 50 . & \text { PPB } & 05 / 19 / 80 \\ \text { LI } & 7 . & 2 . & \text { PPM } & 05 / 19 / 80 \\ \text { LI } & 9 . & 2 . & \text { PPM } & 05 / 19 / 80 \\ \text { TH } & 21.3 & 2.1 & \text { PPM } & 06 / 06 / 80 \\ \text { TH } & 20.9 & 2.1 & \text { PPM } & 06 / 06 / 80 \\ \text { TH } & 21.9 & 2.2 & \text { PPM } & 06 / 06 / 80 \\ U & 6.1 & .6 & \text { PPM } & 07 / 08 / 80 \\ U & 6.1 & .6 & \text { PPM } & 07 / 08 / 80 \\ \text { U } & 6.1 & .6 & \text { PPM } & 07 / 08 / 80 \\ W & 29 . & 4 . & \text { PPM } & 10 / 20 / 80 \\ W & 30 . & 4 . & \text { PPM } & 10 / 20 / 80\end{array}$


TABLE C-1I (cont)

\begin{tabular}{|c|c|c|c|c|c|}
\hline $\begin{array}{l}\text { SAMPLE } \\
\text { NUMBER }\end{array}$ & ANALYSIS & RESULT & UNCERTAINTY & UNITS & $\begin{array}{l}\text { COMPLETION } \\
\text { DATE }\end{array}$ \\
\hline 00583 & -- & $----\cdot$ & 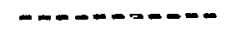 & -...- & $-\cdot$ \\
\hline .00583 & AL & 19.1 & 1. & $\%$ & $03 / 15 / 80$ \\
\hline & $A L$ & 19.6 & 1. & $\%$ & $03 / 15 / 80$ \\
\hline & $A L$ & 19.6 & 1. & $\%$ & $03 / 18 / 80$ \\
\hline & $\mathrm{AL}$ & 19.1 & 1 . & $\%$ & $03 / 18 / 80$ \\
\hline & $\mathrm{AL}$ & 19.7 & 1 . & $\%$ & $03 / 20 / 80$ \\
\hline & $\mathrm{AL}$ & 19.8 & 1. & $\%$ & $03 / 20 / 80$ \\
\hline & $\mathrm{AL}$ & 21.1 & 2.1 & $\%$ & $07 / 25 / 80$ \\
\hline & $A L$ & 19. & 1.9 & $\%$ & $07 / 28 / 80$ \\
\hline & $A L$ & 20.1 & 2. & $\%$ & $10 / 28 / 80$ \\
\hline & AS & 10.7 & 1.1 & PPM & $01 / 18 / 80$ \\
\hline & AS & 11. & 1.1 & PPM & $01 / 18 / 80$ \\
\hline & AS & 12. & 3. & PPM & $07 / 14 / 80$ \\
\hline & AS & 12.6 & 1.3 & PPM & $09 / 22 / 80$ \\
\hline & AS & 13.9 & 1.4 & PPM & $09 / 22 / 80$ \\
\hline & $B A$ & 2000 & 200. & PPM & $04 / 25 / 80$ \\
\hline & $B A$ & 1850. & 190. & PPM & $04 / 30 / 80$ \\
\hline & $B A$ & 1700 . & 170. & PPM & 05/05/80 \\
\hline & $\mathrm{BA}$ & 1810 . & 180. & PPM & $05 / 10 / 80$ \\
\hline & $B A$ & 1620 . & 200. & PPM & $09 / 19 / 80$ \\
\hline & $B A$ & 1810. & 200. & PPM & $09 / 19 / 80$ \\
\hline & BA & 2100 . & 200. & $\begin{array}{l}\text { PPM } \\
\text { PPM }\end{array}$ & $09 / 19 / 80$ \\
\hline & $\mathrm{BA}$ & 2060 . & 200. & PPM & $\begin{array}{l}09 / 19 / 80 \\
10 / 06 / 80\end{array}$ \\
\hline & $\begin{array}{l}B A \\
B A\end{array}$ & $\begin{array}{l}2040 . \\
1830\end{array}$ & $\begin{array}{l}200 . \\
180 .\end{array}$ & $\begin{array}{l}\text { PPM } \\
\text { PPM }\end{array}$ & $\begin{array}{l}10 / 06 / 80 \\
10 / 06 / 80\end{array}$ \\
\hline & $\begin{array}{l}D A \\
B R\end{array}$ & 8000 & 1. & PPM & $09 / 22 / 80$ \\
\hline & $B R$ & 8.3 & 1. & PPM & $09 / 22 / 80$ \\
\hline & CE & 44. & 6. & PPM & $04 / 25 / 80$ \\
\hline & CE & 43. & 6 . & PPM & $04 / 30 / 80$ \\
\hline & $C \overline{C E}$ & 42. & 6 . & PPM & 05/05/80 \\
\hline & $\mathrm{CE}$ & 43. & 6. & PPM & $05 / 10 / 80$ \\
\hline & $\mathrm{CE}$ & 40. & 4. & PPM & $09 / 19 / 80$ \\
\hline & CE & 38. & 4. & PPM & $09 / 19 / 80$ \\
\hline & $\mathrm{CE}$ & 38. & 4. & PPM & 09/19/80 \\
\hline & $\mathrm{CE}$ & 42. & 4. & PPM & $09 / 19 / 80$ \\
\hline & $\mathrm{CE}$ & 39. & 4. & PPM & $10 / 06 / 80$ \\
\hline & $\mathrm{CE}$ & 41. & 4. & PPM & $10 / 06 / 80$ \\
\hline & CE & 38. & 4. & PPM & $11 / 18 / 80$ \\
\hline & $C E$ & 36. & 4. & PPM & $11 / 18 / 80$ \\
\hline & co & 28. & 3. & PPM & $04 / 25 / 80$ \\
\hline & $\mathrm{CO}$ & 29. & 3. & PPM & $04 / 30 / 80$ \\
\hline & $\mathrm{CO}$ & 30. & 3. & PPIM & $05 / 05 / 80$ \\
\hline & CO & 30. & 3. & PPM & $05 / 10 / 80$ \\
\hline & co & 30. & 3. & PPM & $07 / 18 / 80$ \\
\hline & co & 27.4 & 3. & PPM & $07 / 28 / 80$ \\
\hline & CO & 27 . & 3. & PPM & $07 / 28 / 80$ \\
\hline & $\mathrm{CO}$ & 28. & 3. & PPM & $09 / 19 / 80$ \\
\hline & CO & 33. & 3. & PPM & $09 / 19 / 80$ \\
\hline
\end{tabular}


TABLE C-II (cont)

\begin{tabular}{|c|c|c|c|c|c|}
\hline $\begin{array}{l}\text { SAMPLE } \\
\text { NUMBER }\end{array}$ & ANALYSIS & RESULT & UNCERTAINTY & UNITS & $\begin{array}{c}\text { COMPLETION } \\
\text { DATE }\end{array}$ \\
\hline 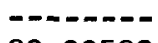 & - - & 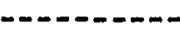 & - n-non & -...- & --non- \\
\hline & $\mathrm{CO}$ & 30. & 3. & PPM & $09 / 19 / 80$ \\
\hline & co & 28. & 3. & PPM & $09 / 19 / 80$ \\
\hline & CO & 30. & 3. & PPM & $09 / 22 / 80$ \\
\hline & $\mathrm{CO}$ & 32. & 3. & PPM & $09 / 22 / 80$ \\
\hline & $\mathrm{CR}$ & 99. & .9 & PPM & $01 / 30 / 80$ \\
\hline & $C R$ & 99. & .9 & PPM & $01 / 30 / 80$ \\
\hline & CR & 104. & 10. & PPM & $04 / 25 / 80$ \\
\hline & $\mathrm{CR}$ & 100. & 10. & PPM & $04 / 30 / 80$ \\
\hline & $\mathrm{CR}$ & 100. & 10. & PPM & $05 / 05 / 80$ \\
\hline & CR & 101. & 10. & PPM & $05 / 10 / 80$ \\
\hline & $\mathrm{CR}$ & 100. & 11. & PPM & $07 / 28 / 80$ \\
\hline & $\mathrm{CR}$ & 105. & 11. & PPM & $07 / 28 / 80$ \\
\hline & CR & 95. & 10. & PPM & $09 / 19 / 80$ \\
\hline & CR & 95. & 10. & PPM & $09 / 19 / 80$ \\
\hline & CR & 97. & 10. & PPM & $09 / 19 / 80$ \\
\hline & $\mathrm{CR}$ & 94. & 10. & PPM & $09 / 19 / 80$ \\
\hline & CR & 100. & 10. & PPM & $09 / 22 / 80$ \\
\hline & CR & 110. & 10. & PPM & $09 / 22 / 80$ \\
\hline & CR & 101. & 10. & PPM & $11 / 18 / 80$ \\
\hline & CR & 92. & 9. & PPM & $11 / 18 / 80$ \\
\hline & CS & 2.2 & .4 & PPM & $04 / 25 / 80$ \\
\hline & CS & 2.3 & .4 & PPM & $04 / 30 / 80$ \\
\hline & CS & 2.4 & .4 & PPM & $05 / 05 / 80$ \\
\hline & CS & 2.2 & .4 & PPM & $05 / 10 / 80$ \\
\hline & $\mathrm{CS}$ & 2.3 & .3 & PPM & $11 / 18 / 80$ \\
\hline & $\mathrm{CS}$ & 2.7 & .4 & PPM & $11 / 18 / 80$ \\
\hline & DY & 2.7 & .6 & PPM & $10 / 28 / 80$ \\
\hline & EU & .89 & .09 & PPM & $04 / 25 / 80$ \\
\hline & EU & .99 & .09 & PPM & $04 / 30 / 80$ \\
\hline & EU & .96 & .09 & PPM & $05 / 05 / 80$ \\
\hline & EU & .93 & .09 & PPM & $05 / 10 / 80$ \\
\hline & EU & 1.08 & .1 & PPM & $09 / 19 / 80$ \\
\hline & EU & .93 & .1 & PPM & $09 / 19 / 80$ \\
\hline & EU & .95 & .1 & PPM & $09 / 19 / 80$ \\
\hline & EU & 1. & .1 & PPM & $09 / 19 / 80$ \\
\hline & EU & 1. & .1 & PPM & $10 / 06 / 80$ \\
\hline & EU & .8 & .1 & PPM & $10 / 06 / 80$ \\
\hline & EU & .99 & .12 & PPM & $11 / 18 / 80$ \\
\hline & $\begin{array}{l}\text { EU } \\
\text { FF }\end{array}$ & .95 & .11 & PPM & $\begin{array}{l}11 / 18 / 80 \\
01 / 30 / 80\end{array}$ \\
\hline & $\begin{array}{l}\mathrm{FE} \\
\mathrm{FE}\end{array}$ & $\begin{array}{l}3.3 \\
3.3\end{array}$ & $\begin{array}{l}.3 \\
.3\end{array}$ & $\begin{array}{l}\% \\
\%\end{array}$ & $\begin{array}{l}01 / 30 / 80 \\
01 / 30 ; 80\end{array}$ \\
\hline & FE & 3.38 & .16 & $\%$ & $04 / 25 / 80$ \\
\hline & $\mathrm{FE}$ & 3.43 & .17 & $\ddot{q}$ & $04 / 30 / 80$ \\
\hline & $\mathrm{FE}$ & 3.33 & .15 & $\ddot{q}$ & $05 / 05 / 80$ \\
\hline & $\mathrm{FE}$ & 3.41 & .16 & $\%$ & $05 / 10 / 80$ \\
\hline & FE & 3.3 & .3 & $\%$ & $07 / 18 / 80$ \\
\hline & $\mathrm{FE}$ & 3.14 & .4 & $\%$ & $07 / 28 / 80$ \\
\hline & $\mathrm{FE}$ & 3.12 & .4 & $\%$ & $07 / 28 / 80$ \\
\hline & $\mathrm{FE}$ & , & 3 & $\%$ & $09 / 19 / 80$ \\
\hline
\end{tabular}




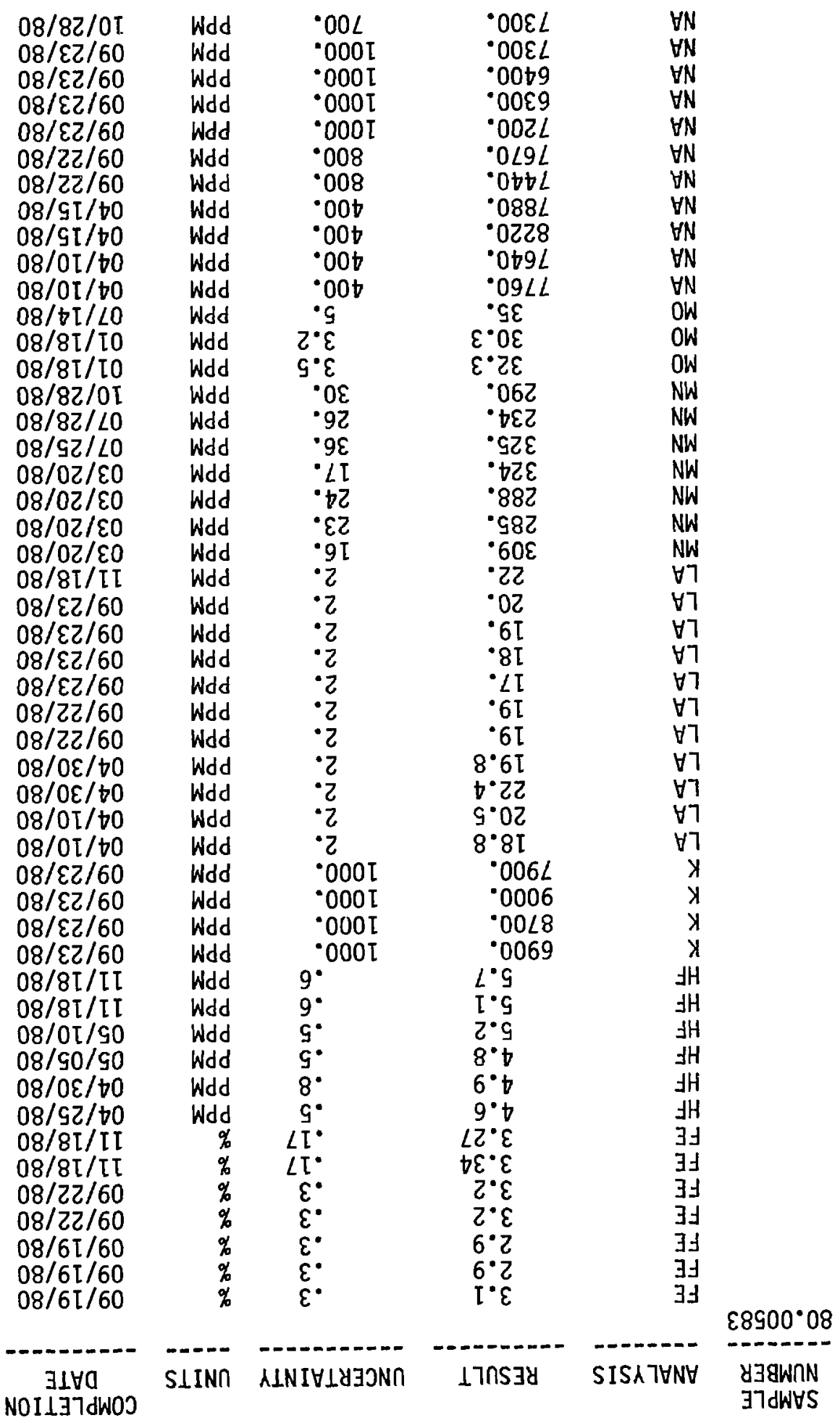


TABLE C-II (cont)

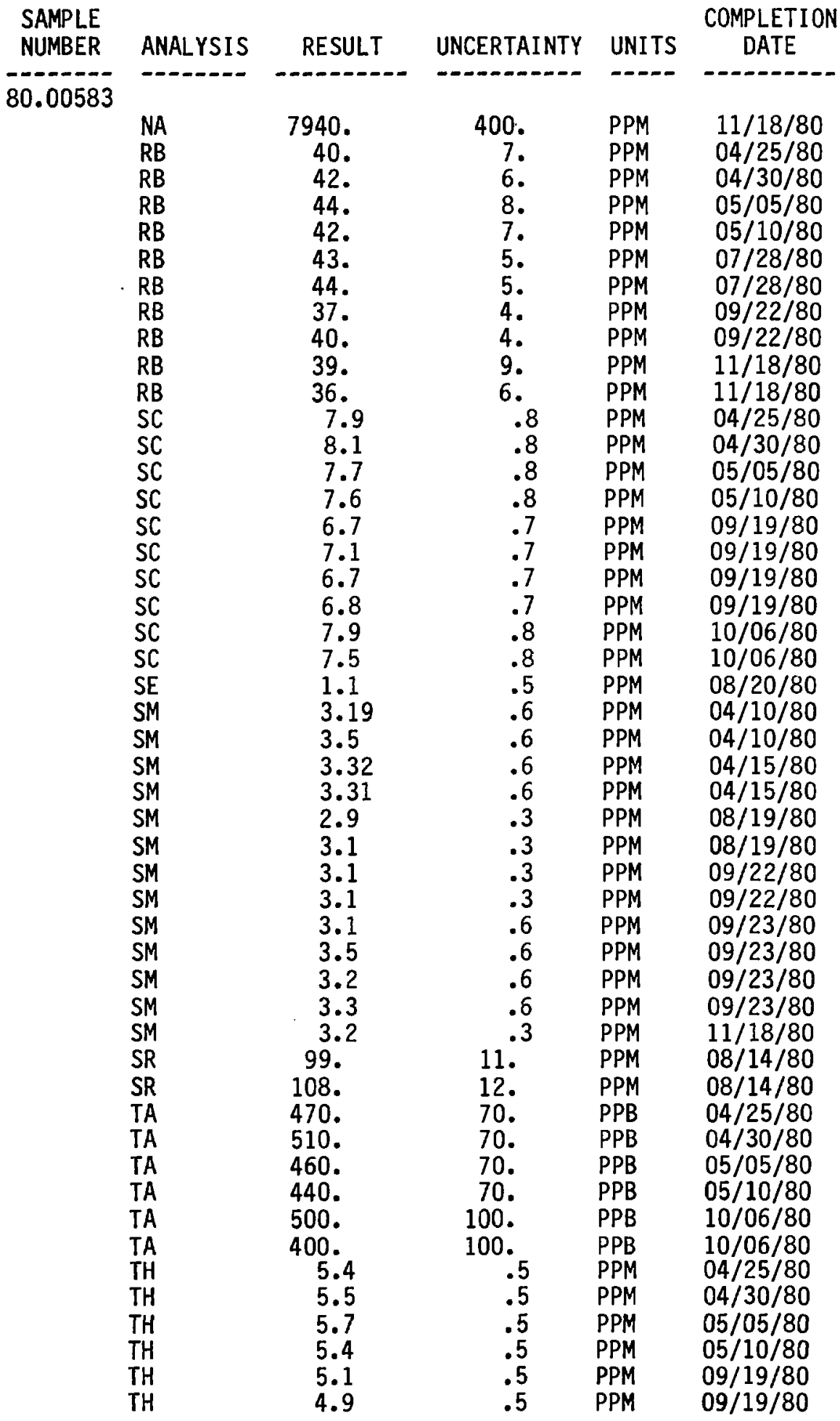


TABLE C-II (cont)

\begin{tabular}{|c|c|c|c|c|c|}
\hline $\begin{array}{l}\text { SAMPLE } \\
\text { NUMBER }\end{array}$ & ANALYSIS & RESULT & UNCERTAINTY & UNITS & $\begin{array}{c}\text { COMPLETION } \\
\text { DATE }\end{array}$ \\
\hline - & - & - & - & $\cdots$ & 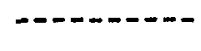 \\
\hline 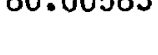 & $T H$ & 4.9 & .5 & PPM & $09 / 19 / 80$ \\
\hline & TH & 5.2 & .5 & PPM & $09 / 19 / 80$ \\
\hline & TH & 5.2 & .5 & PPM & $10 / 06 / 80$ \\
\hline & TH & 5.3 & .5 & PPM & $10 / 06 / 80$ \\
\hline & TH & 5.5 & .6 & PPM & $11 / 18 / 80$ \\
\hline & $T H$ & 5.4 & .6 & PPM & $11 / 18 / 80$ \\
\hline & TI & 2280. & 130. & PPM & $08 / 20 / 80$ \\
\hline & TI & 2290. & 130. & PPM & $08 / 20 / 80$ \\
\hline & $U$ & 2. & .2 & PPM & $07 / 08 / 80$ \\
\hline & $u$ & 2. & .2 & PPM & $07 / 08 / 80$ \\
\hline & u & 2. & .2 & PPM & $07 / 08 / 80$ \\
\hline & u & 1.9 & .3 & PPM & $07 / 14 / 80$ \\
\hline & v & 61. & 19. & PPM & $03 / 15 / 80$ \\
\hline & v & 71 . & 19. & PPM & $03 / 15 / 80$ \\
\hline & v & 61 . & 17. & PPM & $03 / 18 / 80$ \\
\hline & v & 91. & 19. & PPM & $03 / 18 / 80$ \\
\hline & $v$ & 56. & 18. & PPM & $03 / 20 / 80$ \\
\hline & v & 110. & 24. & PPM & $03 / 20 / 80$ \\
\hline & v & 65. & 12. & PPM & $07 / 25 / 80$ \\
\hline & v & 61. & 12. & PPM & $07 / 28 / 80$ \\
\hline & v & 110. & 35 . & PPM & $10 / 28 / 80$ \\
\hline & YB & 2.1 & .4 & PPM & $04 / 25 / 80$ \\
\hline & YB & 2. & .4 & PPM & $04 / 30 / 80$ \\
\hline & YB & 1.6 & .4 & PPM & $05 / 05 / 80$ \\
\hline & YB & 1.7 & .4 & PPM & $05 / 10 / 80$ \\
\hline & YB & 2.4 & .2 & PPM & $10 / 06 / 80$ \\
\hline & YB & 2.1 & .2 & PPM & $10 / 06 / 80$ \\
\hline & YB & 1.4 & .3 & PPM & $11 / 18 / 80$ \\
\hline & YB & 1.6 & .3 & PPM & $11 / 18 / 80$ \\
\hline & $\mathrm{ZN}$ & 31. & 15. & PPM & $07 / 28 / 80$ \\
\hline
\end{tabular}

80.00585

$\begin{array}{lllll}\text { AL } & 16.3 & .8 & \% & 02 / 05 / 80 \\ \text { AL } & 16.2 & .8 & \% & 02 / 05 / 80 \\ \text { AL } & 16.3 & .8 & \% & 02 / 05 / 80 \\ \text { AL } & 16.2 & .8 & \% & 03 / 15 / 80 \\ \text { AL } & 16.1 & .8 & \% & 03 / 15 / 80 \\ \text { AL } & 16.2 & .8 & \% & 03 / 18 / 80 \\ \text { AL } & 16.1 & .8 & \% & 03 / 18 / 80 \\ \text { AL } & 17 . & .9 & \% & 03 / 20 / 80 \\ \text { AL } & 16.5 & .8 . & \% & 03 / 20 / 80 \\ \text { AL } & 15.9 & 1.6 & \% & 07 / 25 / 80 \\ \text { AL } & 18.7 & 1.9 & \% & 07 / 28 / 80 \\ \text { AS } & 340 . & 15 . & \text { PPM } & 07 / 14 / 80 \\ \text { AS } & 335 . & 15 . & \text { PPM } & 07 / 14 / 80 \\ \text { AS } & 326 . & 32 . & \text { PPM } & 08 / 18 / 80 \\ \text { AS } & 320 . & 32 . & \text { PPM } & 08 / 18 / 80 \\ \text { AS } & 330 . & 40 . & \text { PPM } & 08 / 19 / 80 \\ \text { AS } & 360 . & 40 . & \text { PPM } & 08 / 19 / 80\end{array}$


TABLE C-II (cont)

SAMPLE

NUMBER
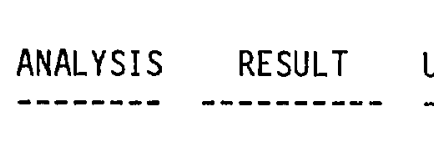

80.00585

AS

AS

$B A$

BA 1290.

$B A \quad 1170$.

BA 1210 .

BA 1380 .

BA 1090 .

$B A \quad 1580$.

BA 1640 .

BA 1190 .

$B A$

CE

CE

CE

CE

CE

CE

CE

CE

CE

CE

CO

CO

CO

CO

$\mathrm{CO}$

$\mathrm{CO}$

$\mathrm{CO}$

CO

$\mathrm{CO}$

$\mathrm{CO}$

$\mathrm{CO}$

CO

CR

1260 .

41.

32.

35.

39.

39.

39.

38.

39.

38.

39.

12.

13.

13.

14.

14.2

14.1

14.

15.

14.

15.

15.

14.

88.

CR

CR

CR

CR

CR

CR

CR

CR

CR

CR

CR

CS

90.

91.

84.

93.

94.

118.

118.

115.

116.

99.

99.

4.7

CS

UNCERTAINTY UNITS

COMPLETION

DATE

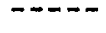

$\begin{array}{lll}\text { 50. } & \text { PPM } & 08 / 19 / 80 \\ \text { 30. } & \text { PPM } & 09 / 22 / 80\end{array}$

30. PPM $09 / 22 / 80$

200. PPM $05 / 15 / 80$

190. PPM $05 / 20 / 80$

200. PPM $05 / 25 / 80$

200. PPM $05 / 30 / 80$

150. PPM $09 / 19 / 80$

150. PPM 09/19/80

150.

150 .

120.

130.

6.

5.

5.

6.

4.

4.

4.

4.

4.

PPM

PPM

PPM

PPM

PPM

PPM

PPM

PPM

PPM

PPM

PPM

PPM

PPM

PPM

PPM

PPM

PPM

PPM

PPM

PPM

PPM

PPM

PPM

PPM

PPM

PPM

PPM

PPM

$09 / 19 / 80$

$09 / 19 / 80$

$10 / 06 / 80$

$10 / 06 / 80$

$05 / 15 / 80$

$05 / 20 / 80$

$05 / 25 / 80$

$05 / 30 / 80$

$09 / 19 / 80$

$09 / 19 / 80$

$09 / 19 / 80$

$09 / 19 / 80$

$10 / 06 / 80$

$10 / 06 / 80$

$05 / 15 / 80$

$05 / 20 / 80$

$05 / 25 / 80$

$05 / 30 / 80$

$07 / 28 / 80$

$07 / 28 / 80$

$09 / 19 / 80$

$09 / 19 / 80$

$09 / 19 / 80$

$09 / 19 / 80$

$09 / 22 / 80$

$09 / 22 / 80$

$05 / 15 / 80$

$05 / 20 / 80$

$05 / 25 / 80$

$05 / 30 / 80$

$07 / 28 / 80$

$07 / 28 / 80$

$09 / 19 / 80$

$09 / 19 / 80$

$09 / 19 / 80$

$09 / 19 / 80$

$09 / 22 / 80$

$09 / 22 / 80$

$05 / 15 / 80$

$05 / 20 / 80$ 


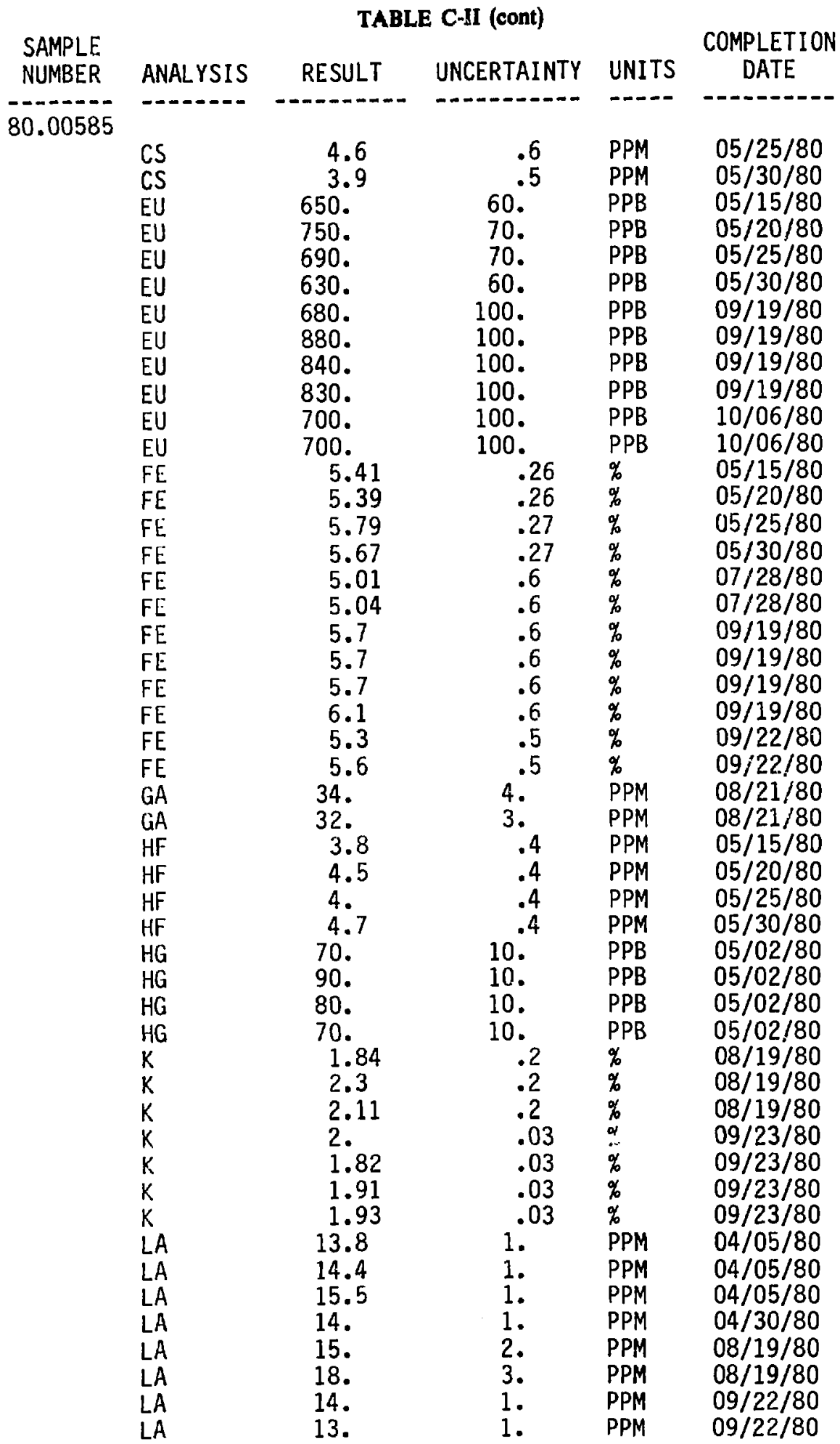


TABLE C-II (cont)

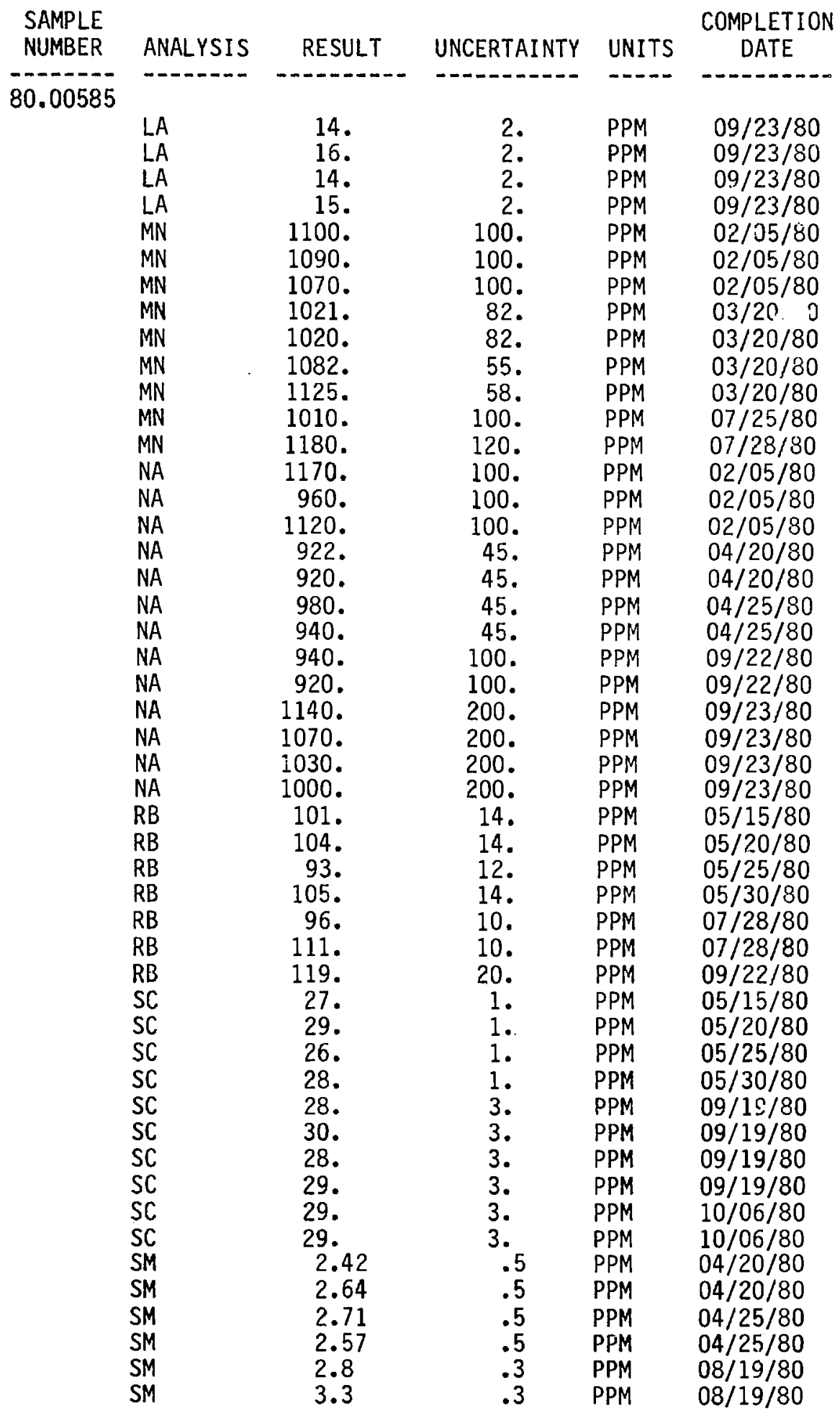


TABLE C-II (cont)

\begin{tabular}{|c|c|c|c|c|c|}
\hline $\begin{array}{l}\text { SAMPLE } \\
\text { NUMBER }\end{array}$ & ANALYSIS & & INCFRTAINTY & UNTTS & $\begin{array}{c}\text { COMPLETION } \\
\text { DATE }\end{array}$ \\
\hline $2-2-2-2$ & $\begin{array}{ll}-2-2-0 \\
\end{array}$ & - & 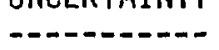 & - & - \\
\hline & SM & 2.3 & .2 & PPM & $09 / 22 / 80$ \\
\hline & SM & 2.3 & .2 & PPM & $09 / 22 / 80$ \\
\hline & SM & 2.8 & .6 & PPM & $09 / 23 / 80$ \\
\hline & SM & 2.9 & .6 & PPM & $09 / 23 / 80$ \\
\hline & SM & 2.6 & .6 & PPM & $09 / 23 / 80$ \\
\hline & SM & 2.8 & .6 & PPM & $09 / 23 / 80$ \\
\hline & SR & 34. & 7. & PPM & $08 / 14 / 80$ \\
\hline & $S R$ & 40. & 8. & PPM & $08 / 14 / 80$ \\
\hline & TA & 390. & 70. & PPB & $05 / 15 / 80$ \\
\hline & TA & 380. & 70. & PPB & $05 / 20 / 80$ \\
\hline & TA & 360. & 70. & PPB & $05 / 25 / 80$ \\
\hline & TA & 330. & 70. & PPB & $05 / 30 / 80$ \\
\hline & TA & 600. & 100. & PPB & $10 / 06 / 80$ \\
\hline & TA & 700. & 100. & PPB & $10 / 06 / 80$ \\
\hline & $\mathrm{TH}$ & 4.8 & .5 & PPM & $05 / 15 / 80$ \\
\hline & $\mathrm{TH}$ & 5.7 & .5 & PPM & $05 / 20 / 80$ \\
\hline & $\mathrm{TH}$ & 5.2 & .5 & PPM & $05 / 25 / 80$ \\
\hline & $\mathrm{TH}$ & 5.1 & .5 & PPM & $05 / 30 / 80$ \\
\hline & $\mathrm{TH}$ & 5.5 & .6 & PPM & $09 / 19 / 30$ \\
\hline & $\mathrm{TH}$ & 5.6 & .6 & PPM & $09 / 19 / 80$ \\
\hline & $\mathrm{TH}$ & 5.6 & .6 & PPM & $09 / 19 / 80$ \\
\hline & $\mathrm{TH}$ & 5.9 & .6 & PPM & $09 / 19 / 80$ \\
\hline & $\mathrm{TH}$ & 5. & .5 & PPM & $10 / 06 / 80$ \\
\hline & $\mathrm{TH}$ & 5. & .5 & PPM & $10 / 06 / 80$ \\
\hline & TI & 5200. & .05 & PPM & $02 / 05 / 80$ \\
\hline & TI & 4800 . & .05 & PPM & $02 / 05 / 80$ \\
\hline & TI & 4900. & .05 & PPM & $02 / 05 / 80$ \\
\hline & TI & 4730 . & 250 & PPM & $08 / 20 / 80$ \\
\hline & TI & 4910. & 260. & PPM & $08 / 20 / 80$ \\
\hline & $U$ & 1.6 & .2 & PPM & $07 / 08 / 80$ \\
\hline & U & 1.5 & .2 & PPM & $07 / 08 / 80$ \\
\hline & U & 1.5 & .2 & PPM & $07 / 08 / 80$ \\
\hline & V & 210 & 30. & PPM & $01 / 22 / 80$ \\
\hline & V & 210. & 30. & PPM & $01 / 22 / 80$ \\
\hline & V & 206. & 20. & PPM & $02 / 05 / 80$ \\
\hline & V & 199. & 20. & PPM & $02 / 05 / 80$ \\
\hline & V & 188. & 20. & PPM & $02 / 05 / 80$ \\
\hline & V & 179. & 25. & PPM & $03 / 15 / 80$ \\
\hline & V & 199. & 27. & PPM & $03 / 15 / 80$ \\
\hline & V & 179. & 25. & PPM & $03 / 18 / 80$ \\
\hline & V & 199. & 27. & PPM & $03 / 18 / 80$ \\
\hline & V & 208. & 32. & PPM & $03 / 20 / 80$ \\
\hline & V & 185. & 23. & PPM & $03 / 20 / 80$ \\
\hline & V & 200. & 23. & PPM & $07 / 25 / 80$ \\
\hline & V & 212 . & 27 . & PPM & $07 / 28 / 80$ \\
\hline & YB & 2.5 & .5 & PPM & $05 / 15 / 80$ \\
\hline & $Y B$ & 2.8 & .5 & PPM & $05 / 20 / 80$ \\
\hline & YB & 2.2 & .5 & PPM & $05 / 25 / 80$ \\
\hline & YB & 2.8 & .5 & PPM & $05 / 30 / 80$ \\
\hline
\end{tabular}


TABLE C-II (cont)

\begin{tabular}{|c|c|c|c|c|c|}
\hline $\begin{array}{l}\text { SAMPLE } \\
\text { NUMBER }\end{array}$ & ANALYSIS & RESULT & UNCERTAINTY & UNITS & $\begin{array}{c}\text { COMPLETION } \\
\text { DATE }\end{array}$ \\
\hline \multicolumn{6}{|l|}{80.00585} \\
\hline & $\begin{array}{l}Y B \\
Y B \\
Z N\end{array}$ & $\begin{array}{c}2.6 \\
3 . \\
158 .\end{array}$ & $\begin{array}{r}.3 \\
40.3\end{array}$ & $\begin{array}{l}\text { PPM } \\
\text { PPM } \\
\text { PPM }\end{array}$ & $\begin{array}{l}10 / 06 / 80 \\
10 / 06 / 80 \\
07 / 28 / 80\end{array}$ \\
\hline \multicolumn{6}{|l|}{80.00589} \\
\hline & $\begin{array}{l}\text { AS } \\
\text { AS } \\
\text { AS } \\
A S \\
A S \\
A S \\
A S \\
\text { BE } \\
B E \\
C D \\
C D \\
C D \\
C D \\
C D \\
C D \\
C R \\
C R \\
P B \\
P B \\
S E \\
S E\end{array}$ & $\begin{array}{l}20 . \\
20 . \\
28 . \\
28 . \\
29 . \\
32 . \\
29 . \\
18 . \\
18 . \\
7 . \\
7 . \\
7 . \\
6 . \\
6.4 \\
5.8 \\
14 . \\
18 . \\
21 . \\
20 . \\
7 . \\
6 .\end{array}$ & $\begin{array}{l}5 . \\
5 . \\
5 . \\
5 . \\
5 . \\
3 . \\
3 . \\
5 . \\
5 . \\
3 . \\
3 . \\
3 . \\
3 . \\
.1 \\
.1 \\
2 . \\
2 . \\
2 . \\
2 . \\
5 . \\
5 .\end{array}$ & $\begin{array}{l}\text { PPB } \\
\text { PPB } \\
\text { PPB } \\
\text { PPB } \\
\text { PPB } \\
\text { PPB } \\
\text { PPB } \\
\text { PPB } \\
\text { PPB } \\
\text { PPB } \\
\text { PPB } \\
\text { PPB } \\
\text { PPB } \\
\text { PPB } \\
\text { PPB } \\
\text { PPB } \\
\text { PPB } \\
\text { PPB } \\
\text { PPB } \\
\text { PPB } \\
\text { PPB }\end{array}$ & $\begin{array}{l}01 / 29 / 80 \\
01 / 29 / 80 \\
01 / 30 / 80 \\
01 / 30 / 80 \\
01 / 30 / 80 \\
06 / 13 / 80 \\
06 / 13 / 80 \\
01 / 29 / 80 \\
01 / 29 / 80 \\
01 / 30 / 80 \\
01 / 30 / 80 \\
01 / 30 / 80 \\
01 / 30 / 80 \\
06 / 11 / 80 \\
06 / 11 / 80 \\
06 / 12 / 80 \\
06 / 12 / 80 \\
06 / 13 / 80 \\
06 / 13 / 80 \\
06 / 13 / 80 \\
06 / 13 ; 30\end{array}$ \\
\hline
\end{tabular}

80.00590

$\begin{array}{lcccc}\text { AS } & 103 . & 10 . & \text { PPB } & 01 / 29 / 80 \\ \text { AS } & 103 . & 10 . & \text { PPB } & 01 / 29 / 80 \\ \text { AS } & 108 . & 11 . & \text { PPB } & 01 / 30 / 80 \\ \text { AS } & 111 . & 11 . & \text { PPB } & 01 / 30 / 80 \\ \text { AS } & 109 . & 11 . & \text { PPB } & 01 / 30 / 80 \\ \text { AS } & 104 . & & P P B & 05 / 27 / 80 \\ \text { AS } & 117 . & & \text { PPB } & 05 / 27 / 80 \\ \text { AS } & 130 . & 3 . & \text { PPB } & 05 / 27 / 80 \\ \text { AS } & 109 . & 3 . & \text { PPB } & 05 / 27 / 80 \\ \text { AS } & 113 . & 3 . & \text { PPB } & 05 / 27 / 80 \\ \text { AS } & 115 . & 3 . & \text { PPB } & 05 / 27 / 80 \\ \text { AS } & 78 . & .8 & \text { PPB } & 05 / 28 / 80 \\ \text { AS } & 97 . & .8 & \text { PPB } & 05 / 28 / 80 \\ \text { AS } & 99 . & 10 . & \text { PPB } & 06 / 02 / 80 \\ \text { AS } & 100 . & 10 . & \text { PPB } & 06 / 02 / 80 \\ \text { AS } & 108 . & 3 . & P P B & 06 / 02 / 80 \\ \text { AS } & 112 . & 12 . & \text { PPB } & 06 / 02 / 80 \\ \text { AS } & 116 . & 12 . & \text { PPB } & 06 / 02 / 80 \\ \text { AS } & 96 . & 10 . & \text { PPB } & 06 / 02 / 80 \\ \text { AS } & 100 . & 10 . & \text { PPB } & 06 / 02 / 80 \\ \text { AS } & 108 . & 3 . & \text { PPB } & 06 / 02 / 80\end{array}$


TABLE C-II (cont)

\begin{tabular}{|c|c|c|c|c|c|}
\hline $\begin{array}{l}\text { SAMPLE } \\
\text { NUMBER }\end{array}$ & ANALYSIS & RESULT & UNCERTAINTY & UNITS & $\begin{array}{c}\text { COMPLETION } \\
\text { DATE }\end{array}$ \\
\hline 80 & $-\cdots-n-m$ & 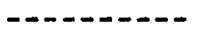 & 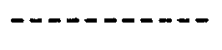 & $\cdots-$ & 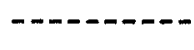 \\
\hline & AS & 112. & 12. & PPB & $06 / 02 / 80$ \\
\hline & AS & 99. & 10. & PPB & $06 / 02 / 80$ \\
\hline & AS & 105. & 10. & PPB & $06 / 04 / 80$ \\
\hline & AS & 111. & 10. & PPB & $06 / 04 / 80$ \\
\hline & AS & 105. & 10. & PPB & $06 / 04 / 80$ \\
\hline & AS & 111. & 10. & PPB & $06 / 04 / 80$ \\
\hline & AS & 113. & 11. & PPB & $06 / 13 / 80$ \\
\hline & AS & 115. & 12. & PPB & $06 / 13 / 80$ \\
\hline & $\mathrm{BE}$ & 73. & 7. & PPB & $01 / 29 / 80$ \\
\hline & $\mathrm{BE}$ & 73. & 7. & PPB & $01 / 29 / 80$ \\
\hline & $\mathrm{CD}$ & 20. & 2. & PPB & $01 / 29 / 80$ \\
\hline & $\mathrm{CD}$ & 20 & 2. & PPB & $01 / 29 / 80$ \\
\hline & CD & 22 . & 3. & PPB & $01 / 30 / 80$ \\
\hline & $\mathrm{CD}$ & 22. & 3. & PPB & $01 / 30 / 80$ \\
\hline & CD & 22. & 3. & PPB & $01 / 30 / 80$ \\
\hline & $C D$ & 19. & 3. & PPB & $01 / 30 / 80$ \\
\hline & CD & 22. & 3. & PPB & $06 / 04 / 80$ \\
\hline & $\mathrm{CO}$ & 26 . & 3. & PPB & $06 / 04 / 80$ \\
\hline & $C D$ & 22. & 3. & PPB & $06 / 04 / 80$ \\
\hline & $C D$ & 30. & 3. & PPB & $06 / 04 / 80$ \\
\hline & $C D$ & 36. & 3. & PPB & $06 / 04 / 80$ \\
\hline & $\mathrm{CD}$ & 21. & 3. & PPB & $06 / 04 / 80$ \\
\hline & $C D$ & 20. & 3. & PPB & $06 / 04 / 80$ \\
\hline & $C D$ & 21. & 3. & PPB & $06 / 04 / 80$ \\
\hline & $C D$ & 18. & 3. & PPB & $06 / 04 / 80$ \\
\hline & $C D$ & 22. & 3. & PPB & $06 / 04 / 80$ \\
\hline & $C D$ & 30. & 3. & PPB & $06 / 04 / 80$ \\
\hline & $C D$ & 20. & 3. & PPB & $06 / 04 / 80$ \\
\hline & $C D$ & 26 . & 3. & PPB & $06 / 04 / 80$ \\
\hline & $C D$ & 21. & 3. & PPB & $06 / 04 / 80$ \\
\hline & $C D$ & 21. & 3. & PPB & $06 / 04 / 80$ \\
\hline & $C D$ & 19. & 3. & PPB & $06 / 04 / 80$ \\
\hline & $C D$ & 22. & 3. & PPB & $06 / 04 / 80$ \\
\hline & $C D$ & 22. & 3. & PPB & $06 / 04 / 80$ \\
\hline & $C D$ & 18. & 3. & PPB & $06 / 04 / 80$ \\
\hline & $\mathrm{CD}$ & 24. & 3. & PPB & $06 / 04 / 80$ \\
\hline & $\mathrm{CD}$ & 24 . & 3. & PPB & $06 / 04 / 80$ \\
\hline & $C D$ & 36. & 3. & PPB & $06 / 04 / 80$ \\
\hline & $C D$ & 24. & 3. & PPB & $06 / 04 / 80$ \\
\hline & $C D$ & 24. & 3. & PPB & $06 / 04 / 80$ \\
\hline & $C D$ & 21 . & 2. & PPB & $06 / 11 / 80$ \\
\hline & $C D$ & 18. & 2. & PPB & $06 / 11 / 80$ \\
\hline & CR & 138. & 15. & PPB & $06 / 12 / 80$ \\
\hline & CR & 145. & 15. & PPB & $06 / 12 / 80$ \\
\hline & PB & 279. & 30. & PPB & $06 / 13 / 80$ \\
\hline & PB & 266. & 30. & PPB & $06 / 13 / 80$ \\
\hline & SE & 26. & 5. & PPB & $06 / 13 / 80$ \\
\hline & SE & 27 . & 5. & PPB & $06 / 13 / 80$ \\
\hline
\end{tabular}


TABLE C-II (cont)

\begin{tabular}{clcccc}
$\begin{array}{c}\text { SAMPLE } \\
\text { NUMBER }\end{array}$ & ANALYSIS & RESULT & UNCERTAINTY & UNITS & $\begin{array}{c}\text { COMPLETION } \\
\text { DATE }\end{array}$ \\
\hline 80.00591 & & & & & \\
& AS & 141. & 15. & PPB & $01 / 29 / 80$ \\
& AS & 141. & 15. & PPB & $01 / 29 / 80$ \\
& AS & 155. & 16. & PPB & $01 / 30 / 80$ \\
& AS & 158. & 16. & PPB & $01 / 30 / 80$ \\
& AS & 170. & 17. & PPB & $01 / 30 / 80$ \\
& AS & 180. & 20. & PPB & $02 / 01 / 80$ \\
& BE & 423. & 42. & PPB & $01 / 29 / 80$ \\
& BE & 423. & 42. & PPB & $01 / 29 / 80$ \\
& CD & 70. & 20. & PPB & $01 / 29 / 80$ \\
& CD & 70. & 20. & PPB & $01 / 29 / 80$ \\
& CD & 83. & 8. & PPB & $01 / 30 / 80$ \\
& CD & 78. & 8. & PPB & $01 / 30 / 80$ \\
& CD & 83. & 8. & PPB & $01 / 30 / 80$ \\
& CD & 72. & 7. & PPB & $01 / 30 / 80$ \\
SE & 48. & 6. & PPB & $08 / 20 / 80$ \\
V & 160. & 16. & PPB & $06 / 02 / 80$
\end{tabular}

80.00592

\begin{tabular}{|c|c|c|c|c|}
\hline $\begin{array}{l}C A \\
C L \\
C L \\
C L \\
C L \\
C L \\
C L \\
C L \\
C L \\
C L \\
C L \\
C L \\
C L \\
C L \\
C L \\
C L \\
C L \\
C L \\
C L \\
C L \\
C L \\
C L \\
C L \\
C L \\
C L \\
C L \\
C 03 \\
C 03 \\
F \\
F\end{array}$ & $\begin{array}{l}21 . \\
29.1 \\
28.5 \\
28.7 \\
28.7 \\
28.9 \\
28.8 \\
27.8 \\
28 . \\
25.1 \\
22.9 \\
25.1 \\
22.9 \\
31.5 \\
29.2 \\
32.7 \\
32.4 \\
32.2 \\
32.5 \\
30.5 \\
28.2 \\
30.9 \\
32.4 \\
30.5 \\
28.8 \\
30.5 \\
16.5 \\
16 . \\
.2 \\
.2\end{array}$ & $\begin{array}{l}2.1 \\
2.9 \\
2.8 \\
2.9 \\
2.9 \\
2.9 \\
2.9 \\
2.8 \\
2.8 \\
2.8 \\
2 . \\
2 . \\
2 . \\
3.1 \\
2.9 \\
3.3 \\
3.3 \\
3.2 \\
3.2 \\
3 . \\
3 . \\
3 . \\
3.2 \\
3 . \\
2.9 \\
3 . \\
1.6 \\
2 . \\
.1 \\
.1\end{array}$ & $\begin{array}{l}\text { PPM } \\
\text { PPM } \\
\text { PPM } \\
\text { PPM } \\
\text { PPM } \\
\text { PPM } \\
\text { PPM } \\
\text { PPM } \\
\text { PPM } \\
\text { PPM } \\
\text { PPM } \\
\text { PPM } \\
\text { PPM } \\
\text { PPM } \\
\text { PPM } \\
\text { PPM } \\
\text { PPM } \\
\text { PPM } \\
\text { PPM } \\
\text { PPM } \\
\text { PPM } \\
\text { PPM } \\
\text { PPM } \\
\text { PPM } \\
\text { PPM } \\
\text { PPM } \\
\text { MG/L } \\
M G / L \\
\text { PPM } \\
\text { PPM }\end{array}$ & $\begin{array}{l}04 / 09 / 80 \\
03 / 17 / 80 \\
03 / 17 / 80 \\
03 / 17 / 80 \\
03 / 20 / 80 \\
03 / 21 / 80 \\
03 / 21 / 80 \\
04 / 02 / 80 \\
04 / 11 / 80 \\
04 / 23 / 80 \\
04 / 23 / 80 \\
04 / 23 / 80 \\
04 / 23 / 80 \\
04 / 29 / 80 \\
04 / 29 / 80 \\
06 / 02 / 80 \\
06 / 02 / 80 \\
06 / 02 / 80 \\
06 / 02 / 80 \\
06 / 11 / 80 \\
07 / 07 / 80 \\
07 / 07 / 80 \\
08 / 04 / 80 \\
10 / 20 / 80 \\
10 / 20 / 80 \\
11 / 07 / 80 \\
11 / 07 / 80 \\
11 / 07 / 80 \\
01 / 11 / 80 \\
01 / 11 / 80\end{array}$ \\
\hline
\end{tabular}


TABLE C-II (cont)

\begin{tabular}{|c|c|c|c|c|c|}
\hline $\begin{array}{l}\text { SAMPLE } \\
\text { NUMBER }\end{array}$ & ANALYSIS & RESULT & UNCERTAINTY & UNITS & $\begin{array}{l}\text { COMPLETION } \\
\text { DATE }\end{array}$ \\
\hline \multicolumn{6}{|l|}{80.60592} \\
\hline 00.00036 & $F$ & .2 & .1 & PPM & $01 / 11 / 80$ \\
\hline & $\mathrm{F}$ & .15 & .01 & PPM & $03 / 17 / 80$ \\
\hline & $\mathrm{F}$ & .26 & .03 & PPM & $03 / 17 / 80$ \\
\hline & $\mathrm{F}$ & .24 & .02 & PPM & $03 / 17 / 80$ \\
\hline & $\mathrm{F}$ & .21 & .02 & PPM & $03 / 20 / 80$ \\
\hline & $\mathrm{F}$ & .2 & .02 & PPM & $03 / 21 / 80$ \\
\hline & $\mathrm{F}$ & .1 & .01 & PPM & $04 / 02 / 80$ \\
\hline & $\mathrm{F}$ & .3 & .03 & PPM & $04 / 11 / 80$ \\
\hline & $\mathrm{F}$ & .2 & .02 & PPM & $04 / 29 / 80$ \\
\hline & $F$ & .1 & .01 & PPM & $04 / 29 / 80$ \\
\hline & $\mathrm{F}$ & .3 & .03 & PPM & $06 / 02 / 80$ \\
\hline & $\mathrm{F}$ & .3 & .03 & PPM & $06 / 02 / 80$ \\
\hline & $\mathrm{F}$ & .3 & .03 & PPM & $06 / 02 / 80$ \\
\hline & $\mathrm{F}$ & .3 & .03 & PPM & $06 / 02 / 80$ \\
\hline & $\mathrm{F}$ & .2 & .02 & PPM & $06 / 11 / 80$ \\
\hline & $\mathrm{F}$ & .1 & .02 & PPM & $07 / 01 / 80$ \\
\hline & $\mathrm{F}$ & .2 & .02 & PPM & $10 / 20 / 80$ \\
\hline & $\mathrm{F}$ & .2 & .02 & PPM & $10 / 20 / 80$ \\
\hline & $\mathrm{F}$ & .28 & .03 & PPM & $11 / 07 / 80$ \\
\hline & HARD & 140. & 14. & PPM * & $11 / 07 / 80$ \\
\hline & HARD & 140. & 14. & PPM * & $11 / 07 / 80$ \\
\hline & K & 3.1 & .3 & PPM & $04 / 09 / 80$ \\
\hline & $M G$ & 5. & .5 & PPM & $04 / 09 / 80$ \\
\hline & NA & 5.2 & .5 & PPM & \\
\hline & $\begin{array}{l}\text { S04 } \\
\text { SO4 }\end{array}$ & $\begin{array}{l}11.1 \\
11.2\end{array}$ & 1.1 & $\begin{array}{l}\text { PPM } \\
\text { PPM }\end{array}$ & $\begin{array}{l}03 / 17 / 80 \\
03 / 17 / 80\end{array}$ \\
\hline & $\begin{array}{l}\text { SO4 } \\
\text { SO4 }\end{array}$ & 11.1 & $\begin{array}{l}1.1 \\
1.1\end{array}$ & $\begin{array}{l}\text { PPM } \\
\text { PPM }\end{array}$ & $\begin{array}{l}03 / 17 / 80 \\
03 / 17 / 80\end{array}$ \\
\hline & S04 & 11.1 & 1.1 & PPM & $03 / 20 / 80$ \\
\hline & SO4 & 11.2 & 1.1 & PPM & $03 / 21 / 80$ \\
\hline & S04 & 10.8 & 1.1 & PPM & $04 / 02 / 80$ \\
\hline & SO4 & 11.1 & 1.1 & PPM & $04 / 29 / 80$ \\
\hline & SO4 & 11.1 & 1.1 & PPM & $04 / 29 / 80$ \\
\hline & SO4 & 11.1 & 1.1 & PPM & $06 / 02 / 80$ \\
\hline & SO4 & 11.2 & 1.1 & PPM & $06 / 02 / 80$ \\
\hline & S04 & 10.8 & 1.1 & PPM & $06 / 02 / 80$ \\
\hline & SO4 & 11.2 & 1.1 & PPM & $06 / 02 / 80$ \\
\hline & S04 & 11.9 & 1.2 & PPM & $06 / 11 / 80$ \\
\hline & $\mathrm{SO} 4$ & 13.1 & 1.3 & PPM & $10 / 20 / 80$ \\
\hline & S04 & 12.8 & 1.3 & PPM & $10 / 20 / 80$ \\
\hline & SO4 & 12.5 & 1.2 & PPM & $11 / 07 / 80$ \\
\hline \multicolumn{6}{|l|}{80.00593} \\
\hline & $C A$ & 68. & 6.8 & & $04 / 09 / 80$ \\
\hline & $C L$ & 82.4 & 8.2 & PPM & $03 / 17 / 80$ \\
\hline & $\mathrm{CL}$ & 81.7 & 8.2 & PPM & $03 / 17 / 80$ \\
\hline & $\begin{array}{l}C L \\
C L\end{array}$ & $\begin{array}{l}83.9 \\
75 .\end{array}$ & $\begin{array}{l}8.4 \\
7.5\end{array}$ & $\begin{array}{l}\text { PPM } \\
\text { PPM }\end{array}$ & $\begin{array}{l}04 / 29 / 80 \\
04 / 29 / 80\end{array}$ \\
\hline & $C L$ & 85.2 & 8.5 & PPM & $05 / 13 / 80$ \\
\hline & $\mathrm{CL}$ & 85.5 & 8.5 & PPM & $05 / 13 / 80$ \\
\hline
\end{tabular}


TABLE C-II (cont)

SAMPLE NUMBER

80.00593
ANALYSIS

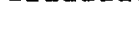

$\mathrm{CL}$

$\mathrm{CL}$

$\mathrm{Cl}$

$\mathrm{CL}$

$\mathrm{F}$

$\mathrm{F}$

F

$\mathrm{F}$

F

$\mathrm{F}$

$\mathrm{F}$

$\mathrm{F}$

$\mathrm{F}$

F

F

K

MG

$\mathrm{SO} 4$

$\mathrm{S} 04$

$\mathrm{S} 04$

$\mathrm{S} 04$

$\mathrm{S} 04$

S04

SO4

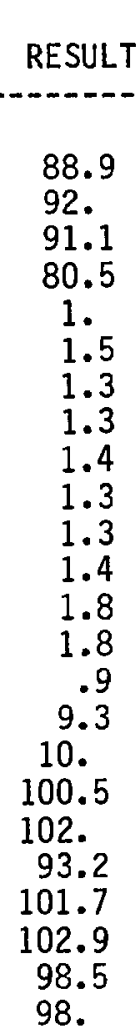

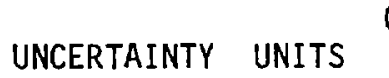

COMPLETION

DATE

$\begin{array}{cc}9 . & \text { PPM } \\ 9.2 & \text { PPM } \\ 9.1 & \text { PPM } \\ 8.1 & \text { PPM } \\ .1 & \text { PPM } \\ .1 & \text { PPM } \\ .03 & \text { PPM } \\ .1 & \text { PPM } \\ .1 & \text { PPM } \\ .1 & \text { PPM } \\ .1 & \text { PPM } \\ .1 & \text { PPM } \\ .2 & \text { PPM } \\ .2 & \text { PPM } \\ .1 & \text { PPM } \\ .9 & \text { PPM } \\ 1.9 & \text { PPM } \\ 10 . & \text { PPM } \\ 10.2 & \text { PPM } \\ 9.3 & \text { PPM } \\ 10.2 & \text { PPM } \\ 10.3 & \text { PPM } \\ 9.8 & \text { PPM } \\ 9.8 & \text { PPM }\end{array}$

$05 / 27 / 80$

$06 / 02 / 80$

$06 / 02 / 80$

$07 / 07 / 80$

$01 / 11 / 80$

$03 / 17 / 80$

$03 / 17 / 80$

$04 / 29 / 80$

$04 / 29 / 80$

$05 / 13 / 80$

$05 / 13 / 80$

$05 / 27 / 80$

$06 / 02 / 80$

$06 / 02 / 80$

$07 / 01 / 80$

$04 / 09 / 80$

$04 / 09 / 80$

$03 / 17 / 80$

$04 / 29 / 80$

$04 / 29 / 80$

$05 / 13 / 80$

$05 / 13 / 80$

$06 / 02 / 80$

$06 / 02 / 80$

80.00594

$\begin{array}{lr}\text { B } & 17 . \\ \text { B } & 19 . \\ \text { B } & 18 . \\ \text { B } & 15 . \\ \text { B } & 18 . \\ \text { SR } & 330 . \\ \text { TI } & 4660 . \\ \text { TI } & 5280 . \\ \text { U } & 1.7 \\ \text { U } & 1.7 \\ \text { U } & 1.7\end{array}$

$\begin{array}{ccc}5 . & \text { PPM } & 12 / 23 / 80 \\ 5 . & \text { PPM } & 12 / 23 / 80 \\ 5 . & \text { PPM } & 12 / 23 / 80 \\ 5 . & \text { PPM } & 12 / 23 / 80 \\ 5 . & \text { PPM } & 12 / 23 / 80 \\ 40 . & \text { PPM } & 12 / 24 / 80 \\ 270 . & \text { PPM } & 01 / 03 / 81 \\ 330 . & \text { PPM } & 01 / 03 / 81 \\ .2 & \text { PPM } & 07 / 08 / 80 \\ .2 & \text { PPM } & 07 / 08 / 80 \\ .2 & \text { PPM } & 07 / 08 / 80\end{array}$

80.00596

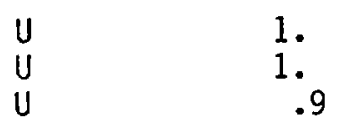
$\begin{array}{ll}.1 & \text { PPM } \\ .1 & \text { PPM } \\ .1 & \text { PPM }\end{array}$
$07 / 08 / 80$
$07 / 08 / 80$
$07 / 08 / 80$

80.00598

$\begin{array}{ll}\text { B } & 23 . \\ B & 26 . \\ B & 24 . \\ B & 22 . \\ B & 25 .\end{array}$

$\begin{array}{lll}\text { 5. } & \text { PPM } & 12 / 23 / 80 \\ \text { 5. } & \text { PPM } & 12 / 23 / 80 \\ \text { 5. } & \text { PPM } & 12 / 23 / 80 \\ \text { 5. } & \text { PPM } & 12 / 23 / 80 \\ \text { 5. } & \text { PPM } & 12 / 23 / 80\end{array}$


TABLE C-II (cont)

SAMPLE

NUMBER
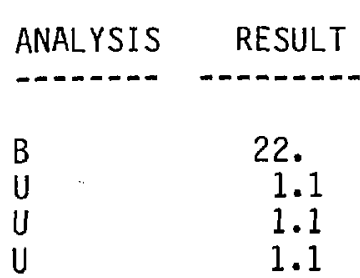

UNC

80.00598

$B$
$U$
$U$
$U$

80.00600

B

B

B

B

U

U

U

U

U

U

U

U

U

U

U

U

U

U

44.
40.
44.
39.

2.3

2.4

2.4

2.4

2.5

2.5

2.4

2.5

2.5

2.4

2.5

2.5

2.4

2. 3

2.4

80.00605

$B$
$B$
$B$
$B$
$B$
$B$
$B$
$B$
$U$
$U$
$U$

58.

57.

55.

59.

48.

50.

57.

55.

1.3

1.4

1.3

$\begin{array}{ccc}5 . & \text { PPM } & 12 / 23 / 80 \\ .1 & \text { PPM } & 07 / 08 / 80 \\ .1 & \text { PPM } & 07 / 08 / 80 \\ .1 & \text { PPM } & 07 / 08 / 80\end{array}$

$\begin{array}{lll}5 . & \text { PPM } & 12 / 23 / 80 \\ 5 . & \text { PPM } & 12 / 23 / 80 \\ 5 . & \text { PPM } & 12 / 23 / 80 \\ 5 . & \text { PPM } & 12 / 23 / 80 \\ .2 & \text { PPM } & 01 / 18 / 80 \\ .2 & \text { PPM } & 01 / 18 / 80 \\ .2 & \text { PPM } & 01 / 18 / 80 \\ .2 & \text { PPM } & 03 / 31 / 80 \\ .3 & \text { PPM } & 03 / 31 / 80 \\ .3 & \text { PPM } & 03 / 31 / 80 \\ .2 & \text { PPM } & 03 / 31 / 80 \\ .3 & \text { PPM } & 03 / 31 / 80 \\ .3 & \text { PPM } & 03 / 31 / 80 \\ .2 & \text { PPM } & 03 / 31 / 80 \\ .3 & \text { PPM } & 03 / 31 / 80 \\ .3 & \text { PPM } & 03 / 31 / 80 \\ .2 & \text { PPM } & 07 / 08 / 80 \\ .2 & \text { PPM } & 07 / 08 / 80 \\ .2 & \text { PPM } & 07 / 08 / 80\end{array}$

$\begin{array}{ccr}5 . & \text { PPM } & 12 / 05 / 80 \\ 5 . & P P M & 12 / 05 / 80 \\ 5 . & P P M & 12 / 05 / 80 \\ 5 . & P P M & 12 / 05 / 80 \\ 5 . & P P M & 12 / 05 / 80 \\ 5 . & P P M & 12 / 23 / 80 \\ 5 . & P P M & 12 / 23 / 80 \\ 5 . & P P M & 12 / 23 / 80 \\ .1 & P P M & 07 / 08 / 80 \\ .1 & P P M & 07 / 08 / 80 \\ .1 & P P M & 07 / 08 / 80\end{array}$

80.00611

$\mathrm{Cl}$

3.08

$.3 \%$

08/05/80

80.00613

$\begin{array}{lllll}C L & 550 . & 60 . & \text { PPM } & 07 / 07 / 80 \\ C L & 580 . & 100 . & \text { PPM } & 08 / 05 / 80 \\ C L & 470 . & 100 . & \text { PPM } & 08 / 05 / 80 \\ C L & 580 . & 100 . & \text { PPM } & 08 / 05 / 80 \\ C L & 570 . & 100 . & P P M & 08 / 05 / 80 \\ C L & 580 . & 100 . & P P M & 08 / 05 / 80\end{array}$


TABLE C-II (cont)

\begin{tabular}{clcccc}
$\begin{array}{c}\text { SAMPLE } \\
\text { NUMBER }\end{array}$ & ANALYSIS & RESULT & UNCERTAINTY & UNITS & $\begin{array}{c}\text { COMPLETION } \\
\text { DATE }\end{array}$ \\
\hline 80.00617 & & & & & \\
& CL & 460. & 50. & PPM & $07 / 07 / 80$ \\
& CL & 460. & 100. & PPM & $08 / 05 / 80$ \\
& CL & 590. & 100. & PPM & $08 / 05 / 80$ \\
& CL & 520. & 100. & PPM & $08 / 05 / 80$ \\
& CL & 480. & 100. & PPM & $08 / 05 / 80$
\end{tabular}

80.00619

\begin{tabular}{|c|c|c|c|c|}
\hline$U$ & 103.9 & 10. & PPB & $01 / 02 / 80$ \\
\hline U & 101.9 & 10 . & PPB & $01 / 02 / 80$ \\
\hline U & 104.8 & 10. & PPB & $01 / 02 / 80$ \\
\hline$U$ & 104. & 10. & PPB & $01 / 02 / 80$ \\
\hline U & 108. & 10. & PPB & $03 / 31 / 80$ \\
\hline U & 105. & 10 . & PPB & $03 / 31 / 80$ \\
\hline U & 102.4 & 10. & PPB & $03 / 31 / 80$ \\
\hline$U$ & 102.9 & 10. & PPB & $03 / 31 / 80$ \\
\hline$U$ & 108. & 10. & PPB & $03 / 31 / 80$ \\
\hline U & 103.9 & 10. & PPB & $03 / 31 / 80$ \\
\hline u & 103.8 & 10 . & PPB & $03 / 31 / 80$ \\
\hline U & 105. & 10 . & PPB & $03 / 31 / 80$ \\
\hline U & 106. & 10 . & PPB & $03 / 31 / 80$ \\
\hline U & 105. & 10. & PPB & $03 / 31 / 80$ \\
\hline U & 103.8 & 10 . & PPB & $03 / 31 / 80$ \\
\hline u & 106. & 10. & PPB & $03 / 31 / 80$ \\
\hline U & 108. & 10. & PPB & $03 / 31 / 80$ \\
\hline U & 105. & 10 . & PPB & $03 / 31 / 80$ \\
\hline U & 102.4 & 10 . & PPB & $03 / 31 / 80$ \\
\hline U & 102.9 & 10 . & PPB & $03 / 31 / 80$ \\
\hline$U$ & 108. & 10. & PPB & $03 / 31 / 80$ \\
\hline U & 103.9 & 10. & PPB & $03 / 31 / 80$ \\
\hline U & 99.9 & 10 . & PPB & $04 / 14 / 80$ \\
\hline U & 101. & 10. & PPB & $06 / 30 / 80$ \\
\hline U & 102. & 10. & PPB & $06 / 30 / 80$ \\
\hline U & 102. & 10 . & PPB & $06 / 30 / 80$ \\
\hline U & 98. & 10. & PPB & $09 / 16 / 80$ \\
\hline U & 94. & 10. & PPB & $09 / 16 / 80$ \\
\hline U & 95. & 10. & PPB & $09 / 16 / 80$ \\
\hline U & 98. & 10. & PPB & $09 / 16 / 80$ \\
\hline U & 96. & 10. & PPB & $09 / 16 / 80$ \\
\hline U & 98. & 10. & PPB & $09 / 16 / 80$ \\
\hline U & 98. & 10 . & PPB & $11 / 19 / 80$ \\
\hline$U$ & 95. & 10 . & PPB & $11 / 19 / 80$ \\
\hline$U$ & 93. & 10 . & PPB & $11 / 19 / 80$ \\
\hline U & 97. & 10 . & PPB & $11 / 19 / 80$ \\
\hline
\end{tabular}

80.00620

$\begin{array}{lllll}\text { NA-22 } & 334 . & 16 . & \text { NCI/L } & 02 / 01 / 80 \\ \text { NA-22 } & 318 . & 13 . & \text { NCI/L } & 03 / 17 / 80\end{array}$


TABLE C-II (cont)

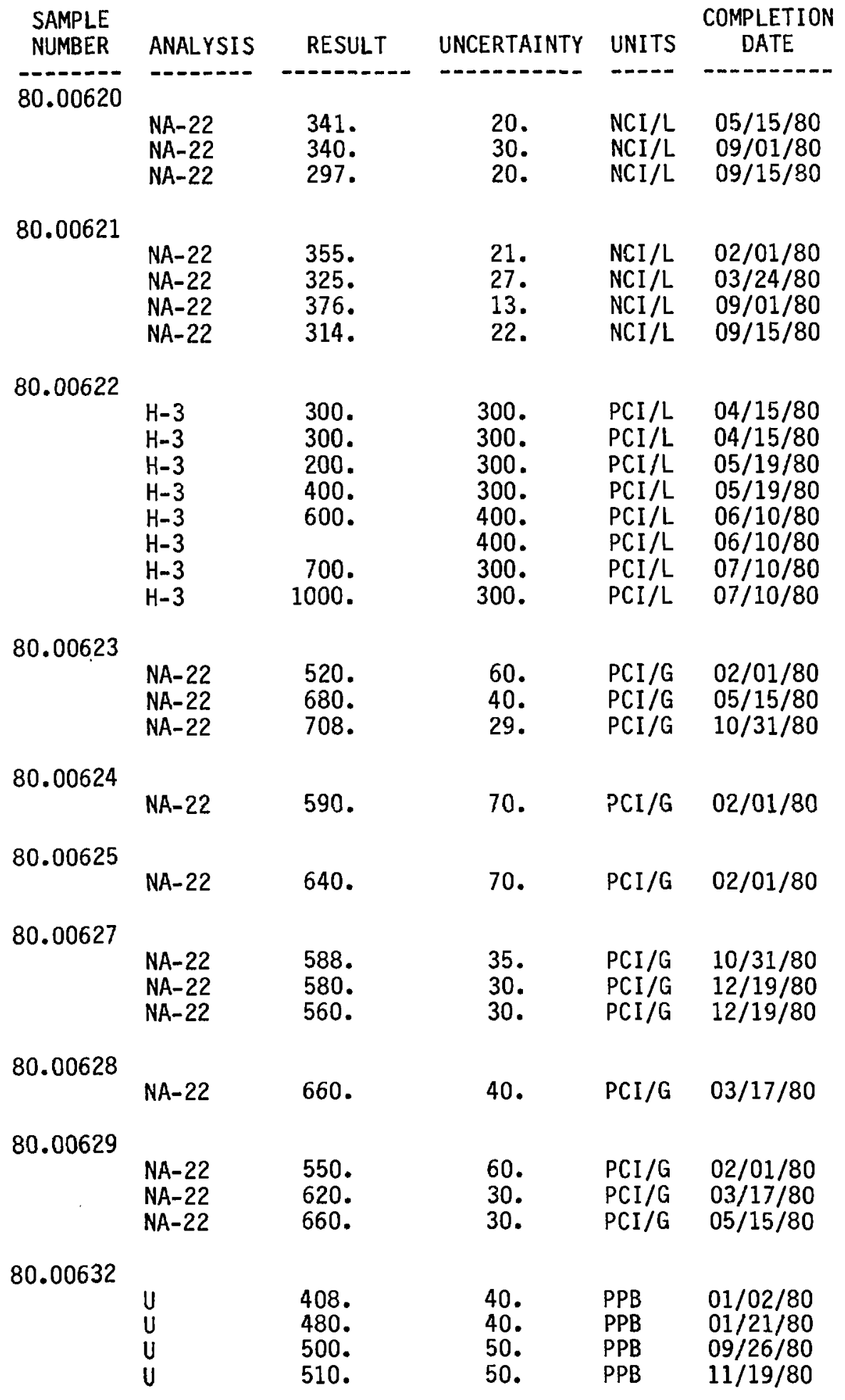


TABLE C-II (cont)

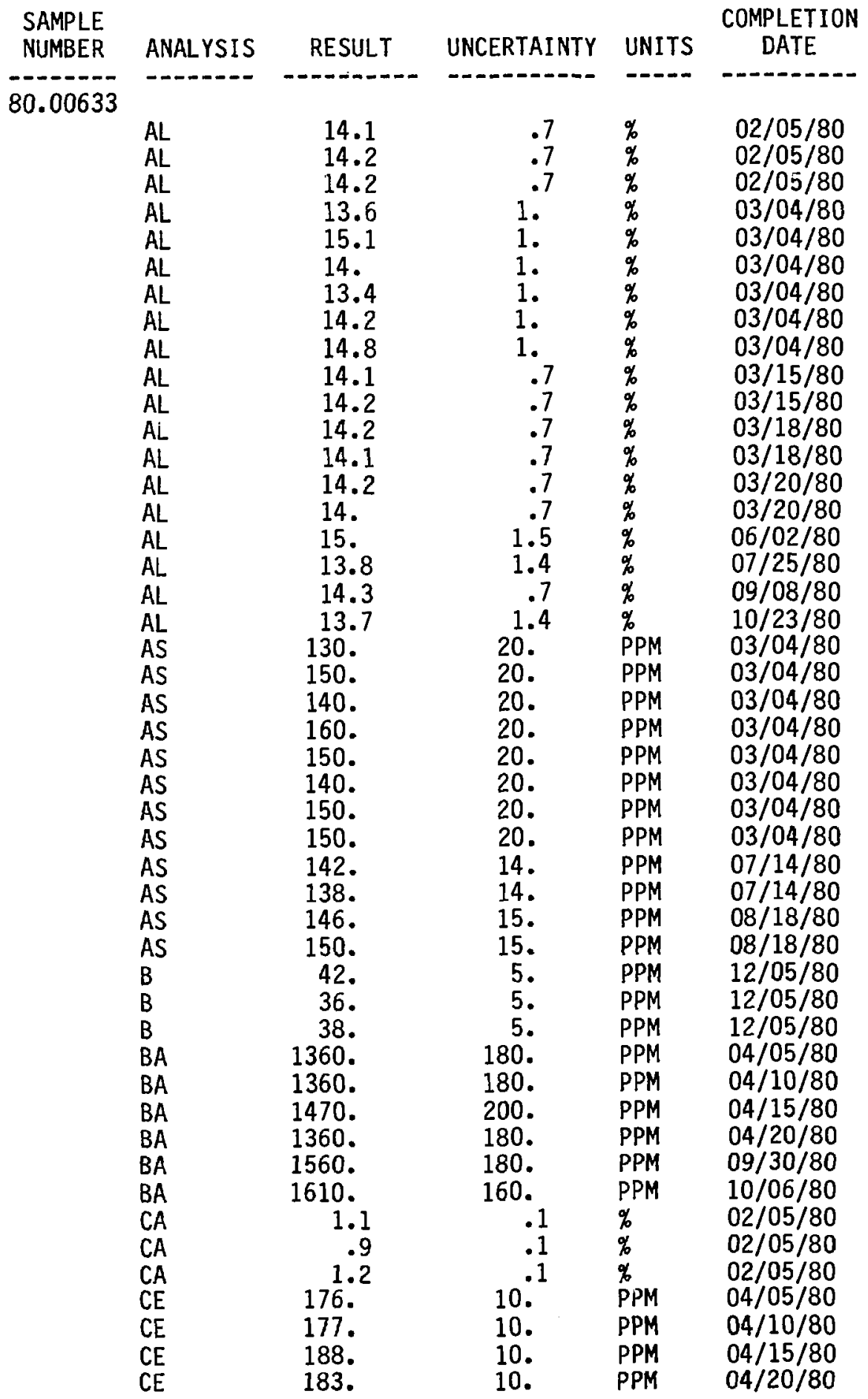


TABLE C-II (cont)

\begin{tabular}{|c|c|c|c|c|c|}
\hline $\begin{array}{l}\text { SAMPLE } \\
\text { NUMBER }\end{array}$ & ANALYSIS & RESULT & UNCERTAINTY & UNITS & $\begin{array}{l}\text { COMPLETION } \\
\text { DATE }\end{array}$ \\
\hline 80.00633 & $---\infty-\infty$ & - & -m-n-m-n & $-\ldots-$ & $\ldots+\ldots$ \\
\hline & CE & 178. & 18. & PPM & $11 / 18 / 80$ \\
\hline & $\mathrm{CE}$ & 176. & 18. & PPM & $11 / 18 / 80$ \\
\hline & $\mathrm{CO}$ & 51. & 5. & PPM & $03 / 20 / 80$ \\
\hline & $\mathrm{CO}$ & 56. & 5. & PPM & $03 / 20 / 80$ \\
\hline & $\mathrm{CO}$ & $\begin{array}{l}47 . \\
46 .\end{array}$ & $\begin{array}{l}5 . \\
5\end{array}$ & $\begin{array}{l}\text { PPM } \\
\text { PPM }\end{array}$ & $\begin{array}{l}03 / 20 / 80 \\
03 / 20 / 80\end{array}$ \\
\hline & $\begin{array}{l}\mathrm{CO} \\
\mathrm{CO}\end{array}$ & $\begin{array}{l}46 . \\
42 .\end{array}$ & $\begin{array}{l}5 . \\
5 .\end{array}$ & $\begin{array}{l}\text { PPM } \\
\text { PPM }\end{array}$ & $\begin{array}{l}03 / 20 / 80 \\
03 / 20 / 80\end{array}$ \\
\hline & $\mathrm{CO}$ & 46. & 5. & PPM & $03 / 20 / 80$ \\
\hline & $\mathrm{CO}$ & 50. & 5. & PPM & 03/20/80 \\
\hline & $\mathrm{CO}$ & 47. & 5. & PPM & $03 / 20 / 80$ \\
\hline & $\mathrm{CO}$ & 44. & 5. & PPM & $03 / 20 / 80$ \\
\hline & $\mathrm{CO}$ & 48. & 5. & PPM & $03 / 20 / 80$ \\
\hline & $\mathrm{CO}$ & 50. & 5. & PPM & $03 / 20 / 80$ \\
\hline & $\mathrm{CO}$ & 51. & 5. & PPM & $03 / 20 / 80$ \\
\hline & CO & 48. & 5. & PPM & $03 / 20 / 80$ \\
\hline & $\mathrm{CO}$ & 47. & 5. & PPM & $03 / 20 / 80$ \\
\hline & $\mathrm{CO}$ & 50. & 5. & PPM & $03 / 20 / 80$ \\
\hline & $\mathrm{CO}$ & 48. & 5. & PPM & $03 / 20 / 80$ \\
\hline & $\mathrm{CO}$ & 52. & 5. & PPM & $03 / 20 / 80$ \\
\hline & $\mathrm{CO}$ & 43. & 4. & PPM & $04 / 05 / 80$ \\
\hline & $\mathrm{CO}$ & 43. & 4. & PPM & $04 / 10 / 80$ \\
\hline & $\mathrm{CO}$ & 44. & 4. & PPM & $04 / 15 / 80$ \\
\hline & $\mathrm{CO}$ & 43. & 4. & PPM & $04 / 20 / 80$ \\
\hline & $\mathrm{CO}$ & 48. & 5. & PPM & $09 / 22 / 80$ \\
\hline & $\mathrm{CO}$ & 51. & 5. & PPM & $09 / 22 / 80$ \\
\hline & $\mathrm{CO}$ & 43. & 2. & PPM & $09 / 30 / 80$ \\
\hline & $C R$ & 180. & 20. & PPM & $03 / 20 / 80$ \\
\hline & $C R$ & 220. & 20. & PPM & $03 / 20 / 80$ \\
\hline & $C R$ & 220. & 20. & PPM & $03 / 20 / 80$ \\
\hline & $C R$ & 180. & 20. & PPM & $03 / 20 / 80$ \\
\hline & $C R$ & 180. & 20. & PPM & $03 / 20 / 80$ \\
\hline & CR & 175. & 20. & PPM & $03 / 20 / 80$ \\
\hline & $C R$ & 200 & 20. & PPM & $03 / 20 / 80$ \\
\hline & $C R$ & 240. & 20. & PPM & $03 / 20 / 80$ \\
\hline & $C R$ & 180. & 20. & PPM & $03 / 20 / 80$ \\
\hline & CR & 180. & 20. & PPM & $03 / 20 / 80$ \\
\hline & $C R$ & 210. & 20. & PPM & $03 / 20 / 80$ \\
\hline & CR & 205. & 20. & PPM & $03 / 20 / 80$ \\
\hline & CR & 210. & 20. & PPM & $03 / 20 / 80$ \\
\hline & CR & 200. & 20. & PPM & $03 / 20 / 80$ \\
\hline & $C R$ & 220. & 20. & PPM & $03 / 20 / 80$ \\
\hline & $C R$ & 170. & 20. & PPM & $03 / 20 / 80$ \\
\hline & CR & 194. & 20. & PPM & $04 / 05 / 80$ \\
\hline & $\mathrm{CR}$ & 200. & 20. & PPM & $04 / 10 / 80$ \\
\hline & CR & 189. & 20. & PPM & $04 / 15 / 80$ \\
\hline & CR & 192. & 20. & PPM & $04 / 20 / 80$ \\
\hline & CR & 217. & 21. & PPM & $09 / 22 / 80$ \\
\hline & $C R$ & 209. & 21. & PPM & $09 / 22 / 80$ \\
\hline & CR & 192. & 10. & PPM & $09 / 30 / 80$ \\
\hline
\end{tabular}


TABLE C-II (cont)

\begin{tabular}{|c|c|c|c|c|c|}
\hline $\begin{array}{l}\text { SAMPLE } \\
\text { NUMBER }\end{array}$ & ANALYSIS & RESULT & UNCERTAINTY & UNITS & $\begin{array}{c}\text { COMPLETION } \\
\text { DATE }\end{array}$ \\
\hline 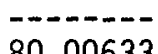 & -- & - & 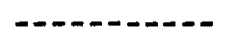 & $\cdots$ & n \\
\hline & $C R$ & 179. & 18. & PPM & $11 / 18 / 80$ \\
\hline & $\mathrm{CR}$ & 191. & 19. & PPM & $11 / 18 / 80$ \\
\hline & CS & 11. & 1.5 & PPM & $04 / 05 / 80$ \\
\hline & $\mathrm{CS}$ & 10.1 & 1.4 & PPM & $04 / 10 / 80$ \\
\hline & CS & 10.6 & 1.5 & PPM & $04 / 15 / 80$ \\
\hline & $\mathrm{CS}$ & 10.5 & 1.5 & PPM & $04 / 20 / 80$ \\
\hline & CS & 10.6 & 1.2 & PPM & $09 / 30 / 80$ \\
\hline & $\mathrm{CS}$ & 10.4 & 1.2 & PPM & $11 / 18 / 80$ \\
\hline & $\operatorname{cs}$ & 10.1 & 1.2 & PPM & $11 / 18 / 80$ \\
\hline & DY & 14.1 & 2.1 & PPM & $10 / 23 / 80$ \\
\hline & DY & 14.9 & 1.5 & PPM & $10 / 28 / 80$ \\
\hline & EU & 3.8 & .4 & PPM & $04 / 05 / 80$ \\
\hline & EU & 3.9 & .4 & PPM & $04 / 10 / 80$ \\
\hline & EU & 3.6 & .4 & PPM & $04 / 15 / 80$ \\
\hline & EU & 3.4 & .4 & PPM & $04 / 20 / 80$ \\
\hline & EU & 3.6 & .4 & PPM & $09 / 30 / 80$ \\
\hline & $E U$ & 4. & .4 & PPM & $10 / 06 / 80$ \\
\hline & EU & 3.9 & .4 & PPM & $10 / 06 / 80$ \\
\hline & EU & 3.4 & .4 & PPM & $11 / 18 / 80$ \\
\hline & EU & 4. & .4 & PPM & $11 / 18 / 80$ \\
\hline & $\mathrm{FE}$ & 9.3 & .9 & $\%$ & $03 / 20 / 80$ \\
\hline & $\mathrm{FE}$ & 10.1 & 1. & $\%$ & 03/20/80 \\
\hline & $F E$ & 9.2 & .9 & $\%$ & $03 / 20 / 80$ \\
\hline & $\mathrm{FE}$ & 10.1 & 1. & $\%$ & 03/20/80 \\
\hline & $\begin{array}{l}\mathrm{FE} \\
\mathrm{FE}\end{array}$ & 8.6 & .9 & $\%$ & $03 / 20 / 80$ \\
\hline & $\mathrm{FE}$ & $\begin{array}{l}9.2 \\
9 .\end{array}$ & 99 & $\begin{array}{l}\% \\
\%\end{array}$ & $\begin{array}{l}03 / 20 / 80 \\
03 / 20 / 80\end{array}$ \\
\hline & $\mathrm{FE}$ & 9.2 & .9 & $\%$ & $03 / 20 / 80$ \\
\hline & $F E$ & 8.8 & .9 & $\%$ & $03 / 20 / 80$ \\
\hline & FE & 8.9 & .9 & $\%$ & $03 / 20 / 80$ \\
\hline & $\mathrm{FE}$ & $\begin{array}{r}9.9 \\
10.2\end{array}$ & $\begin{array}{l}1 . \\
1 .\end{array}$ & $\%$ & $03 / 20 / 80$ \\
\hline & $\mathrm{FE}$ & $\begin{array}{r}10.2 \\
8.4\end{array}$ & 1.8 & $\begin{array}{l}\% \\
\%\end{array}$ & $\begin{array}{l}03 / 20 / 80 \\
03 / 20 / 80\end{array}$ \\
\hline & $\mathrm{FE}$ & 9.9 & 1. & $\%$ & $03 / 20 / 80$ \\
\hline & $\mathrm{FE}$ & 9.4 & .9 & $\%$ & $03 / 20 / 80$ \\
\hline & $\mathrm{FE}$ & 10. & 1. & $\%$ & $03 / 20 / 80$ \\
\hline & $\mathrm{FE}$ & 9.53 & .46 & $\%$ & $04 / 05 / 80$ \\
\hline & $\begin{array}{l}\mathrm{FE} \\
\mathrm{FE}\end{array}$ & $\begin{array}{l}9.18 \\
9.34\end{array}$ & $\begin{array}{l}.45 \\
.45\end{array}$ & $\begin{array}{l}\% \\
\%\end{array}$ & $\begin{array}{l}04 / 10 / 80 \\
04 / 15 / 80\end{array}$ \\
\hline & FE & 9.1 & .45 & $\%$ & $04 / 20 / 80$ \\
\hline & $\mathrm{FE}$ & 9.6 & .9 & $\%$ & $09 / 22 / 80$ \\
\hline & FE & 9.2 & .9 & $\%$ & $09 / 22 / 80$ \\
\hline & $\mathrm{FE}$ & 9.16 & .47 & $\%$ & $09 / 30 / 80$ \\
\hline & GA & 56. & 6 & PPM & $08 / 21 / 80$ \\
\hline & GA & 56. & 6 . & PPM & $08 / 21 / 80$ \\
\hline & HF & 7.7 & .7 & PPM & $04 / 05 / 80$ \\
\hline & HF & 7.5 & .7 & PPM & $04 / 10 / 80$ \\
\hline & $\mathrm{HF}$ & 7.4 & .7 & PPM & $04 / 15 / 80$ \\
\hline & HF & 7.7 & .7 & PPM & $04 / 20 / 80$ \\
\hline
\end{tabular}


TABLE C-II (cont)

\begin{tabular}{|c|c|c|c|c|c|}
\hline $\begin{array}{l}\text { SAMPLE } \\
\text { NUMBER }\end{array}$ & ANALYSIS & RESULT & UNCERTAINTY & UNITS & $\begin{array}{l}\text { COMPLETION } \\
\text { DATE }\end{array}$ \\
\hline 80.00633 & $\cdots$ & - & - - & $-\cdots-$ & --- \\
\hline & $\mathrm{HF}$ & 7.8 & .8 & PPM & $09 / 30 / 80$ \\
\hline & K & 1.87 & .3 & $\%$ & $03 / 04 / 80$ \\
\hline & $\hat{k}$ & 1.79 & .3 & $\%$ & $03 / 04 / 80$ \\
\hline & $\ddot{k}$ & 1.76 & .3 & $\%$ & $03 / 04 / 80$ \\
\hline & $k$ & 2. & .3 & $\%$ & $03 / 04 / 80$ \\
\hline & $k$ & 1.91 & .3 & $\%$ & $03 / 04 / 80$ \\
\hline & $k$ & 1.8 & .3 & $\%$ & $03 / 04 / 80$ \\
\hline & k & 1.79 & .3 & $\%$ & $03 / 04 / 80$ \\
\hline & $k$ & 1.87 & .3 & $\%$ & $03 / 04 / 80$ \\
\hline & $k$ & 1.97 & .4 & $\%$ & $09 / 22 / 80$ \\
\hline & $k$ & 2.03 & .4 & $\%$ & $09 / 22 / 80$ \\
\hline & LA & 73. & 10. & PPM & $03 / 04 / 80$ \\
\hline & LA & 84. & 10. & PPM & $03 / 04 / 80$ \\
\hline & LA & 79. & 10. & PPM & $03 / 04 / 80$ \\
\hline & LA & 78. & 10. & PPM & $03 / 04 / 80$ \\
\hline & LA & 81. & 10. & PPM & $03 / 04 / 80$ \\
\hline & LA & 84. & 10. & PPM & $03 / 04 / 80$ \\
\hline & LA & 90. & 10. & PPM & $03 / 04 / 80$ \\
\hline & LA & 81. & 10. & PPM & $03 / 04 / 80$ \\
\hline & LA & 84. & 8. & PPM & $04 / 15 / 80$ \\
\hline & LA & 81. & 8. & PPM & $04 / 15 / 80$ \\
\hline & $\begin{array}{l}\text { LA } \\
\text { LA }\end{array}$ & $\begin{array}{l}82 . \\
83 .\end{array}$ & $\begin{array}{l}8 . \\
8 .\end{array}$ & $\begin{array}{l}\text { PPM } \\
\text { PPM }\end{array}$ & $\begin{array}{l}04 / 20 / 80 \\
04 / 20 / 80\end{array}$ \\
\hline & LA & 80. & 8. & PPM & $\begin{array}{l}04 / 20 / 80 \\
04 / 25 / 80\end{array}$ \\
\hline & $L A$ & 87. & 8. & PPM & $04 / 25 / 80$ \\
\hline & LA & 86. & 9. & PPM & $09 / 08 / 80$ \\
\hline & LA & 85. & 8. & PPM & $09 / 08 / 80$ \\
\hline & $L A$ & 81. & 8. & PPM & $09 / 22 / 80$ \\
\hline & LA & 83. & 8. & PPM & $09 / 22 / 80$ \\
\hline & LA & 88. & 9. & PPM & $11 / 18 / 80$ \\
\hline & MN & 190. & 10. & PPM & $02 / 05 / 80$ \\
\hline & MN & 187. & 10. & PPM & $02 / 05 / 80$ \\
\hline & MN & 185. & 10. & PPM & $02 / 05 / 80$ \\
\hline & MN & 300. & 30. & PPM & $03 / 04 / 80$ \\
\hline & MN & 280. & 30. & PPM & $03 / 04 / 80$ \\
\hline & MN & 270 & 30. & PPM & 03/04/80 \\
\hline & MN & 290. & 30. & PPM & $03 / 04 / 80$ \\
\hline & MN & 166. & 30. & PPM & $03 / 04 / 80$ \\
\hline & MN & 191. & 30. & PPM & $03 / 04 / 80$ \\
\hline & MN & 163. & 30. & PPM & $03 / 04 / 80$ \\
\hline & MN & 270. & 30. & PPM & $03 / 04 / 80$ \\
\hline & MN & 160. & 30. & PPM & $03 / 04 / 80$ \\
\hline & MN & 164. & 30. & PPM & $03 / 04 / 80$ \\
\hline & MN & 280. & 30. & PPM & $03 / 04 / 80$ \\
\hline & MN & 180. & 10. & PPM & $03 / 20 / 80$ \\
\hline & MN & 185. & 15. & PPM & $03 / 20 / 80$ \\
\hline & MN & 180. & 15. & PPM & $03 / 20 / 80$ \\
\hline & MN & 178. & 10. & PPM & $03 / 20 / 80$ \\
\hline & MN & 187. & 23. & PPM & $07 / 25 / 80$ \\
\hline
\end{tabular}


TABLE C-II (cont)

\begin{tabular}{|c|c|c|c|c|c|}
\hline $\begin{array}{l}\text { SAMPLE } \\
\text { NUMBER }\end{array}$ & ANALYSIS & RESULT & UNCERTAINTY & UNITS & $\begin{array}{c}\text { COMPLETION } \\
\text { DATE }\end{array}$ \\
\hline 80.00633 & $\ldots$ & $-\ldots-$ & 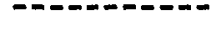 & $-\ldots-$ & 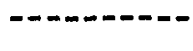 \\
\hline & $M N$ & 182. & 10. & PPM & $09 / 08 / 80$ \\
\hline & MN & 185. & 20. & PPM & $10 / 23 / 80$ \\
\hline & $N A$ & 1900. & 200. & PPM & $03 / 04 / 80$ \\
\hline & $\mathrm{NA}$ & 1740 . & 200. & PPM & $03 / 04 / 80$ \\
\hline & NA & 1680. & 200. & PPM & $03 / 04 / 80$ \\
\hline & NA & 1710. & 200. & PPM & $03 / 04 / 80$ \\
\hline & NA & 1640. & 200. & PPM & $03 / 04 / 80$ \\
\hline & NA & 1600. & 200. & PPM & $03 / 04 / 80$ \\
\hline & NA & 1670. & 200. & PPM & $03 / 04 / 80$ \\
\hline & NA & 1790. & 200. & PPM & $03 / 04 / 80$ \\
\hline & NA & 1750. & 80. & PPM & $04 / 01 / 80$ \\
\hline & NA & 1640. & 80. & PPM & $04 / 01 / 80$ \\
\hline & $N A$ & 1680. & 80. & PPM & $04 / 05 / 80$ \\
\hline & NA & 1710. & 80. & PPM & $04 / 05 / 80$ \\
\hline & NA & 1710. & 80. & PPM & $09 / 08 / 80$ \\
\hline & NA & 1730. & 90. & PPM & $09 / 08 / 80$ \\
\hline & NA & 1940. & 200 . & PPM & $09 / 22 / 80$ \\
\hline & NA & 1900. & 200 & PPM & $09 / 22 / 80$ \\
\hline & NA & 1730 & 90. & PPM! & $11 / 18 / 80$ \\
\hline & & $\begin{array}{l}130 . \\
110 .\end{array}$ & $\begin{array}{l}25 . \\
25 .\end{array}$ & PPM & $03 / 20 / 80$ \\
\hline & $\begin{array}{l}R B \\
R B\end{array}$ & $\begin{array}{l}110 . \\
130 .\end{array}$ & $\begin{array}{l}25 . \\
25 .\end{array}$ & $\begin{array}{l}\text { PPM } \\
\text { PPM }\end{array}$ & $\begin{array}{l}03 / 20 / 80 \\
03 / 20 / 80\end{array}$ \\
\hline & RB & 130. & 25. & PPM & $\begin{array}{l}03 / 20 / 80 \\
03 / 20 / 80\end{array}$ \\
\hline & RB & 150. & 30. & PPM & $03 / 20 / 80$ \\
\hline & RB & 130. & 25 . & PPM & $03 / 20 / 80$ \\
\hline & $\mathrm{RB}$ & 105. & 25 . & PPM & $03 / 20 / 80$ \\
\hline & RB & 130. & 25 . & PPM & $03 / 20 / 80$ \\
\hline & $\mathrm{RB}$ & 110. & 25. & PPM & $03 / 20 / 80$ \\
\hline & $\mathrm{RB}$ & 140. & 25. & PPM & $03 / 20 / 80$ \\
\hline & RB & 145. & 30 . & PPM & $03 / 20 / 80$ \\
\hline & $\mathrm{RB}$ & 140. & 25. & PPM & $03 / 20 / 80$ \\
\hline & $\mathrm{RB}$ & 160. & 30. & PPM & $03 / 20 / 80$ \\
\hline & $\mathrm{RB}$ & 120. & 25. & PPM & $03 / 20 / 80$ \\
\hline & RB & 115. & 25. & PPM & $03 / 20 / 80$ \\
\hline & $\mathrm{RB}$ & 160. & 30. & PPM & $03 / 20 / 80$ \\
\hline & $\mathrm{RB}$ & 131. & 16. & PPM & $04 / 05 / 80$ \\
\hline & $\mathrm{RB}$ & 132. & 16. & PPM & $04 / 10 / 80$ \\
\hline & RB & 144. & 16. & PPM & $04 / 15 / 80$ \\
\hline & $\begin{array}{l}R B \\
R B\end{array}$ & 172. & 20. & PPM & $04 / 20 / 80$ \\
\hline & $\begin{array}{l}\mathrm{RB} \\
\mathrm{RB}\end{array}$ & $\begin{array}{l}131 . \\
145 .\end{array}$ & $\begin{array}{l}16 . \\
17 .\end{array}$ & $\begin{array}{l}\text { PPM } \\
\text { PPM }\end{array}$ & \\
\hline & $\mathrm{RB}$ & 133. & 8. & $\begin{array}{l}\text { PPM } \\
\text { PPM }\end{array}$ & $\begin{array}{l}09 / 30 / 80 \\
11 / 18 / 80\end{array}$ \\
\hline & $\mathrm{RB}$ & 129. & 15. & PPM & $11 / 18 / 80$ \\
\hline & SC & 42. & 2. & PPM & $04 / 05 / 80$ \\
\hline & SC & 41. & 2. & PPM & $04 / 10 / 80$ \\
\hline & SC & 42. & 2. & PPM & $04 / 15 / 80$ \\
\hline & SC & 40. & 2. & PPM & $04 / 20 / 80$ \\
\hline & $\mathrm{SC}$ & 37.5 & 1.9 & PPM & $09 / 30 / 80$ \\
\hline & SC & & 1 & PPM & $10 / 06 / 80$ \\
\hline
\end{tabular}


TABLE C-II (cont)

SAMPLE NUMBER

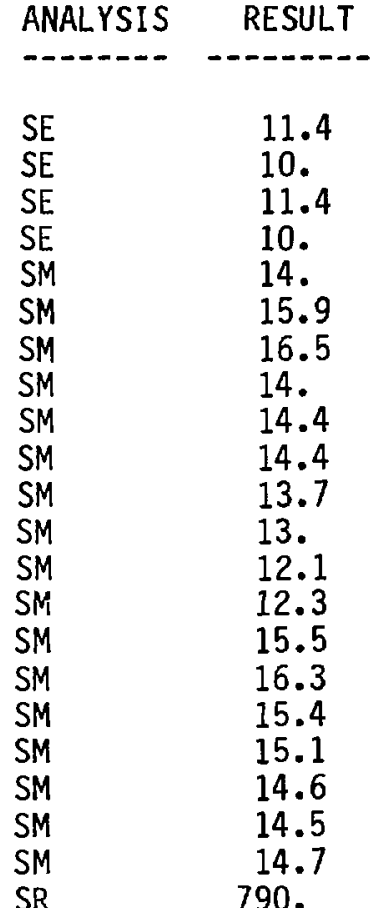

80.00633

SR

TA

TA

TA

TA

TB

TB

TB

TB

TH

TH

TH

TH

$\mathrm{TH}$

TH

TH

TH

TH

TH

TH

TH

TH

TH

TH

TH

TH

790.

750.

1.9

2.

1.9

1.6

2.4

2.3

3.

3.3

25.2

23.7

25.2

27.2

24.5

24.1

24.2

25.2

23.8

24.2

22.7

25.1

25.

25.

24.

24.

24.6

TH

24.1

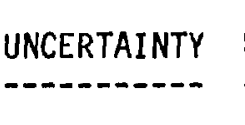

UNITS

COMPLETION DATE

DATE

1. PPM $01 / 30 / 80$

1. PPM $01 / 30 / 80$

1. PPM $01 / 30 / 80$

1. PPM $01 / 30 / 80$

2. PPM $03 / 04 / 80$

2. PPM $03 / 04 / 80$

2. PPM $03 / 04 / 80$

2. PPM 03/04/80

2. PPM 03/04/80

2. PPM 03/04/80

2. PPM $03 / 04 / 80$

2. PPM 03/04/80

2. PPM 04/01/80

2. PPM $04 / 01 / 80$

2. PPM $04 / 05 / 80$

2. PPM $04 / 05 / 80$

1.6 PPM 09/08/80

1.5 PPM $09 / 08 / 80$

1.5 PPM 09/22/80

1.5 PPM 09/22/80

1.5 PPM $11 / 18 / 80$

80. PPM $08 / 14 / 80$

80. PPM 08/14/80

$.2 \quad P P M \quad 04 / 05 / 80$

.2 PPM 04/10/80

.2 PPM 04/15/80

.2 PPM 04/20/80

.3 PPM 04/05/80

.3 PPM 04/10/80

.3 PPM 04/15/80

.4 PPM 04/26/80

1. PPM $02 / 01 / 80$

1. PPM $02 / 01 / 80$

1. PPM $02 / 01 / 80$

1. PPM $02 / 10 / 80$

1. PPM $02 / 10 / 80$

1. PPM $02 / 10 / 80$

1. PPM $02 / 20 / 80$

1. PPM $02 / 20 / 80$

1. PPM $02 / 20 / 80$

1. PPM $03 / 01 / 80$

1. PPM $03 / 01 / 80$

1. PPM $03 / 01 / 80$

1. PPM 04/05/80

1. PPM $04 / 10 / 80$

1. PPM $04 / 15 / 80$

1. PPM $04 / 20 / 80$

2.5 PPM $10 / 06 / 80$

2.4 PPM $11 / 18 / 80$ 
TABLE C-II (cont)

\begin{tabular}{|c|c|c|c|c|c|}
\hline $\begin{array}{l}\text { SAMPLE } \\
\text { NUMBER }\end{array}$ & ANALYSIS & RESULT & UNCERTAINTY & UNITS & $\begin{array}{c}\text { COMPLETION } \\
\text { DATE }\end{array}$ \\
\hline $80---1--$ & ------- & $--m-\infty-n$ & 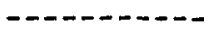 & $-\cdots-$ & --n----- \\
\hline & $\mathrm{TH}$ & 23.9 & 2.4 & PPM & $11 / 18 / 80$ \\
\hline & $\mathrm{TH}$ & 25.9 & 1.5 & PPM & $12 / 20 / 80$ \\
\hline & $\mathrm{TH}$ & 24.3 & 1.4 & PPM & $12 / 24 / 80$ \\
\hline & TI & 8900. & 900. & PPM & $02 / 05 / 80$ \\
\hline & TI & 7200 . & 900. & PPM & $02 / 05 / 80$ \\
\hline & TI & 7500. & 900. & PPM & $02 / 05 / 80$ \\
\hline & TI & 8290. & 430. & PPM & $08 / 20 / 80$ \\
\hline & TI & 8540. & 450. & PPM & $08 / 20 / 80$ \\
\hline & TI & 7770. & 450. & PPM & $01 / 03 / 81$ \\
\hline & TI & 8030. & 460. & PPM & $01 / 03 / 81$ \\
\hline & $U$ & 11.1 & 1. & PPM & $03 / 31 / 80$ \\
\hline & U & 11.1 & 1. & PPM & $03 / 31 / 80$ \\
\hline & U & 11.1 & 1. & PPM & $03 / 31 / 80$ \\
\hline & $U$ & 10.3 & 1. & PPM & $07 / 08 / 80$ \\
\hline & U & 10.5 & 1. & PPM & $07 / 08 / 80$ \\
\hline & U & 10.5 & 1. & PPM & $07 / 08 / 80$ \\
\hline & U & 10.3 & 1. & PPM & $07 / 08 / 80$ \\
\hline & U & 10.3 & 1. & PPM & $07 / 08 / 80$ \\
\hline & U & 10.2 & 1. & PPM & $09 / 26 / 80$ \\
\hline & U & 10.2 & 1 . & PPN & $11 / 19 / 80$ \\
\hline & V & 253. & 25. & PPM & $02 / 05 / 80$ \\
\hline$\cdots$ & V & 255 & 25. & PPM & $02 / 05 / 80$ \\
\hline & V & 258. & 25. & PPM & $02 / 05 / 80$ \\
\hline & V & 304. & 33. & PPM & $03 / 15 / 80$ \\
\hline & V & 328. & 38. & PPM & $03 / 15 / 80$ \\
\hline & V & 328. & 38. & PPM & $03 / 18 / 80$ \\
\hline & V & 304. & 33. & PPM & $03 / 18 / 80$ \\
\hline & V & 287. & 34. & PPM & $03 / 20 / 80$ \\
\hline & V & 324. & 37. & PPM & $03 / 20 / 80$ \\
\hline & $V$ & 320. & 30. & PPM & $06 / 02 / 80$ \\
\hline & V & 278. & 30. & PPM & $07 / 25 / 80$ \\
\hline & V & 290. & 31. & PPM & $09 / 08 / 80$ \\
\hline & $W$ & 4.9 & .8 & PPM & $08 / 18 / 80$ \\
\hline & $W$ & 5.9 & 1.1 & PPM & $08 / 18 / 80$ \\
\hline & $W$ & 5.6 & .8 & PPM & $10 / 20 / 80$ \\
\hline & $W$ & 5.2 & .8 & PPM & $10 / 20 / 80$ \\
\hline & $Y B$ & 8.2 & 1.3 & PPM & $11 / 18 / 80$ \\
\hline
\end{tabular}

80.00637

$\begin{array}{rrrll}\text { CS }-137 & 1020 . & 90 . & \text { PCI/L } & 05 / 01 / 80 \\ \text { CS-137 } & 990 . & 60 . & \text { PCI/L } & 05 / 01 / 80 \\ \text { CS-137 } & 994 . & 94 . & \text { PCI/L } & 11 / 20 / 80 \\ \text { CS-137 } & 1001 . & 57 . & \text { PCI/L } & 11 / 20 / 80 \\ \text { CS-137 } & 1077 . & 45 . & \text { PCI/L } & 11 / 20 / 80 \\ \text { CS-137 } & 1155 . & 50 . & \text { PCI/L } & 11 / 20 / 80\end{array}$

80.00638

$\begin{array}{llrll}\mathrm{CS}-137 & 1000 . & 60 . & \mathrm{PCI} / \mathrm{L} & 05 / 01 / 80 \\ \mathrm{CS}-137 & 1070 . & 100 . & \mathrm{PCI} / \mathrm{L} & 05 / 01 / 80\end{array}$


TABLE C-II (cont)

\begin{tabular}{llcccc}
$\begin{array}{c}\text { SAMPLE } \\
\text { NUMBER }\end{array}$ & ANALYSIS & RESULT & UNCERTAINTY & UNITS & $\begin{array}{c}\text { COMPLETION } \\
\text { DATE }\end{array}$ \\
\hline 80.00638 & & - & & & \\
& CS-137 & 1000. & 40. & PCI/L & $09 / 15 / 80$ \\
& CS-137 & 1024. & 84. & PCI/L & $11 / 20 / 80$ \\
& CS-137 & 1047. & 87. & PCI/L & $11 / 20 / 80$ \\
& CS-137 & 992. & 85. & PCI/L & $11 / 20 / 80$ \\
& CS-137 & 1188. & 89. & PCI/L & $12 / 18 / 80$ \\
& CS-137 & 1023. & 90. & PCI/L & $12 / 29 / 80$
\end{tabular}

80.00639

$\begin{array}{rr}\text { CS-137 } & 970 \\ \text { CS-137 } & 1050 \\ \text { CS-137 } & 996 \\ \text { CS-137 } & 929 \\ \text { CS-137 } & 935\end{array}$

90. PCI/L $05 / 01 / 80$ 90. PCI/L $\quad 09 / 15 / 80$ 61. PCI/L $11 / 20 / 80$ 104. PCI/L $12 / 18 / 80$ 105. $\mathrm{PCI} / \mathrm{L} \quad 12 / 29 / 80$

80.00640

CS-137

CS-137

CS-137

89
.75

.09

.09

$P C I / G$

$P C I / G$

$08 / 01 / 80$

.7

$.06 \mathrm{PCI} / \mathrm{G} \quad 09 / 01 / 80$

$08 / 01 / 80$

80.00641

CS-137

.86

$.08 \quad \mathrm{PCI} / \mathrm{G} \quad 08 / 01 / 80$

$.07 \quad \mathrm{PCI} / \mathrm{G} \quad 08 / 01 / 80$

$.07 \mathrm{PCI} / \mathrm{G} \quad 09 / 01 / 80$

80.00642

CS-137

.88

.09

.05

PCI/G 08/01/80

$\mathrm{PCI} / \mathrm{G} \quad 08 / 01 / 80$

CS-137

.68

$.05 \mathrm{PCI} / \mathrm{G}$

09/01/80

80.00643

$\begin{array}{ll}\text { AS } & 72 . \\ \text { MO } & 91 . \\ \text { PB } & 25 . \\ \text { ZN } & 71 .\end{array}$

5. PPB

5. $\mathrm{PPB}$

2. $\quad \mathrm{PPB}$

1.5 PPB

$10 / 30 / 80$

$10 / 30 / 80$

$10 / 30 / 80$

71.

$10 / 30 / 80$

80.00649

$\begin{array}{lc}\text { AS } & 25 . \\ \text { BE } & 20 . \\ \text { BE } & 22 . \\ \text { BE } & 21 . \\ \text { BE } & 26 . \\ \text { CD } & 7.7 \\ \text { CD } & 8 . \\ \text { CD } & 7 . \\ \text { CD } & 7 . \\ \text { CD } & 7 . \\ \text { CD } & 6 . \\ C R & 4 . \\ C R & 4 .\end{array}$

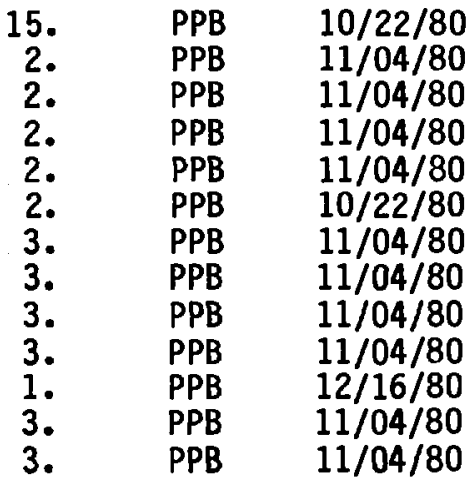


TABLE C-II (cont)

\begin{tabular}{cccccc}
$\begin{array}{c}\text { SAMPLE } \\
\text { NUMBER }\end{array}$ & ANALYSIS & RESULT & UNCERTAINTY & UNITS & COMPLETION \\
\hline 80.00649 & & & & & \\
& CR & 6. & 3. & PPB & $11 / 04 / 80$ \\
& CR & 4. & 3. & PPB & $11 / 04 / 80$ \\
& CR & 5. & 2. & PPB & $12 / 16 / 80$ \\
& PB & 39. & 5. & PPB & $12 / 16 / 80$ \\
& ZN & 8. & 2. & PPB & $12 / 16 / 80$
\end{tabular}

80.00650

\begin{tabular}{|c|c|c|c|c|}
\hline $\begin{array}{l}A S \\
A S \\
B E \\
B E \\
B E \\
C D \\
C D \\
C D \\
C D \\
C D \\
C D \\
C R \\
C R \\
C R \\
C R \\
C R \\
C R \\
C U \\
C U \\
M N \\
P B \\
P B \\
S E \\
Z N \\
Z N\end{array}$ & $\begin{array}{r}158 . \\
156 . \\
260 . \\
229 . \\
283 . \\
58 . \\
66 . \\
61 . \\
65 . \\
62 . \\
62 . \\
250 . \\
295 . \\
295 . \\
293 . \\
292 . \\
417 . \\
421 . \\
428 . \\
518 . \\
417 . \\
376 . \\
52 . \\
516 . \\
514 .\end{array}$ & $\begin{array}{l}15 . \\
30 . \\
10 . \\
10 . \\
10 . \\
2 . \\
3 . \\
3 . \\
3 . \\
3 . \\
1 . \\
10 . \\
10 . \\
10 . \\
10 . \\
10 . \\
41 . \\
30 . \\
48 . \\
50 . \\
30 . \\
35 . \\
10 . \\
30 . \\
45 .\end{array}$ & $\begin{array}{l}P P B \\
P P B \\
P P B \\
P P B \\
P P B \\
P P B \\
P P B \\
\text { PPB } \\
\text { PPB } \\
\text { PPB } \\
\text { PPB } \\
\text { PPB } \\
\text { PPB } \\
\text { PPB } \\
\text { PPB } \\
\text { PPB } \\
\text { PPB } \\
\text { PPB } \\
\text { PPB } \\
\text { PPB } \\
\text { PPB } \\
\text { PPB } \\
\text { PPB } \\
\text { PPB } \\
\text { PPB }\end{array}$ & $\begin{array}{l}10 / 22 / 80 \\
12 / 16 / 80 \\
11 / 04 / 80 \\
11 / 04 / 80 \\
11 / 04 / 80 \\
10 / 22 / 80 \\
11 / 04 / 80 \\
11 / 04 / 80 \\
11 / 04 / 80 \\
11 / 04 / 80 \\
12 / 16 / 80 \\
11 / 04 / 80 \\
11 / 04 / 80 \\
11 / 04 / 80 \\
11 / 04 / 80 \\
11 / 04 / 80 \\
12 / 16 / 80 \\
12 / 16 / 80 \\
12 / 16 / 80 \\
12 / 16 / 80 \\
12 / 16 / 80 \\
12 / 16 / 80 \\
12 / 16 / 80 \\
12 / 16 / 80 \\
12 / 16 / 80\end{array}$ \\
\hline
\end{tabular}

80.00651

$\begin{array}{lrrll}\text { AS } & 56 . & 15 . & \text { PPB } & 10 / 22 / 80 \\ \text { AS } & 56 . & 10 . & \text { PPB } & 12 / 16 / 80 \\ \text { BE } & 173 . & 10 . & \text { PPB } & 11 / 04 / 80 \\ \text { BE } & 185 . & 10 . & \text { PPB } & 11 / 04 / 80 \\ \text { BE } & 179 . & 10 . & \text { PPB } & 11 / 04 / 80 \\ \text { BE } & 185 . & 10 . & \text { PPB } & 11 / 04 / 80 \\ \text { CD } & 22 . & 2 . & \text { PPB } & 10 / 22 / 80 \\ C D & 29 . & 3 . & \text { PPB } & 11 / 04 / 80 \\ C D & 34 . & 3 . & \text { PPB } & 11 / 04 / 80 \\ C D & 32 . & 3 . & \text { PPB } & 11 / 04 / 80 \\ \text { CD } & 30 . & 3 . & \text { PPB } & 11 / 04 / 80 \\ \text { CD } & 28 . & 10 . & \text { PPB } & 12 / 16 / 80 \\ \text { CR } & 65 . & 3 . & \text { PPB } & 11 / 04 / 80 \\ C R & 64 . & 3 . & \text { PPB } & 11 / 04 / 80 \\ \text { CR } & 60 . & 3 . & \text { PPB } & 11 / 04 / 80\end{array}$


TABLE C-II (cont)

SAMPLE NUMBER 80.00651

CR

$C R$

CU

CU

MN

$P B$

PB

SE

$\mathrm{ZN}$

$2 N$

\begin{tabular}{ll} 
ANALYSIS & RESULT \\
\hline CR & \\
CR & 62. \\
CU & 85. \\
CU & 36. \\
MN & 36. \\
PB & 46. \\
PB & 27. \\
SE & 99. \\
ZN & 19. \\
ZN & 25. \\
& 28.
\end{tabular}

80.00652

80.00653
$C A$
$C A$
$C A$
$C A$
$C A$

HARD

$K$

K

K

K

K

MG

MG

MG

$M G$

$M G$

NA

NA

NA

NA

NA

$\begin{array}{ll}C A & 5.4 \\ C A & 5 . \\ C A & 6 . \\ C A & 5.7 \\ C A & 4.9 \\ C L & 19.1 \\ C L & 19.4 \\ C L & 19.2 \\ C L & 19.4 \\ C L & 19.4 \\ C L & 19.4 \\ C L & 19.2 \\ C L & 19.1 \\ C L & 19.2\end{array}$

41.9

41.

41.

39.

39.8

136.

11.

9.5

9.1

10.4

9.7

8.7

8.1

7.1

8.6

9.6

47.

48.

43.5

48.3

45.9

5.4

5.

5.

4.9

19.1

19.4

19.4

19.4

19.4

19.1

CL
COMPLETION

DATE

$\begin{array}{ll} & \\ P P B & 11 / 04 / 80 \\ \text { PPB } & 12 / 16 / 80 \\ \text { PPB } & 12 / 16 / 80 \\ \text { PPB } & 12 / 16 / 80 \\ \text { PPB } & 12 / 16 / 80 \\ \text { PPB } & 12 / 16 / 80 \\ \text { PPB } & 12 / 16 / 80 \\ \text { PPB } & 12 / 16 / 80 \\ \text { PPB } & 12 / 16 / 80 \\ \text { PPB } & 12 / 16 / 80\end{array}$

3. PPB $11 / 04 / 80$

6. $\quad$ PPB $12 / 16 / 80$

2. $P P B \quad 12 / 16 / 80$

2. $\mathrm{PPB} 12 / 16 / 80$

2. $\mathrm{PPB} 12 / 16 / 80$

3. $P P B \quad 12 / 16 / 80$

9. $\mathrm{PPB} 12 / 16 / 80$

10. PPB $12 / 16 / 80$

2. $P P B \quad 12 / 16 / 80$

3. PPB $12 / 16 / 80$

4.2 PPM 08/29/80

2. PPM $10 / 30 / 80$

2. PPM $10 / 30 / 80$

4. PPM $11 / 18 / 80$

$.4 \quad \mathrm{PPM} \quad 12 / 16 / 80$

$13.6 \quad \mathrm{MG} / \mathrm{L} \quad 10 / 20 / 80$

1.1 PPM 08/29/80

$.7 \quad$ PPM $10 / 30 / 80$

.7 PPM $10 / 30 / 80$

$.5 \quad$ PPM $11 / 18 / 80$

.3 PPM $12 / 16 / 80$

.2 PPM 10/30/80

.2 PPM $10 / 30 / 80$

1. PPM $11 / 18 / 80$

.5 PPM $12 / 16 / 80$

.8 PPM $12 / 16 / 80$

1. PPM $10 / 30 / 80$

1. PPM $10 / 30 / 80$

.5 PPM $11 / 18 / 80$

2. PPM $12 / 16 / 80$

1.8 PPM $12 / 16 / 80$

$.5 \quad$ PPM $08 / 29 / 80$

2. PPM $10 / 30 / 80$

2. PPM $10 / 30 / 80$

.1 PPM $11 / 18 / 80$

.4 PPM $12 / 16 / 80$

2. PPM 08/05/80

2. PPM $08 / 05 / 80$

2. PPM $08 / 06 / 80$

2. PPM $08 / 07 / 80$

2. PPM $08 / 07 / 80$

2. PPM $08 / 08 / 80$

2. PPM $08 / 18 / 80$

2. PPM $08 / 19 / 80$

2. PPM $08 / 20 / 80$ 
TABLE C-II (cont)

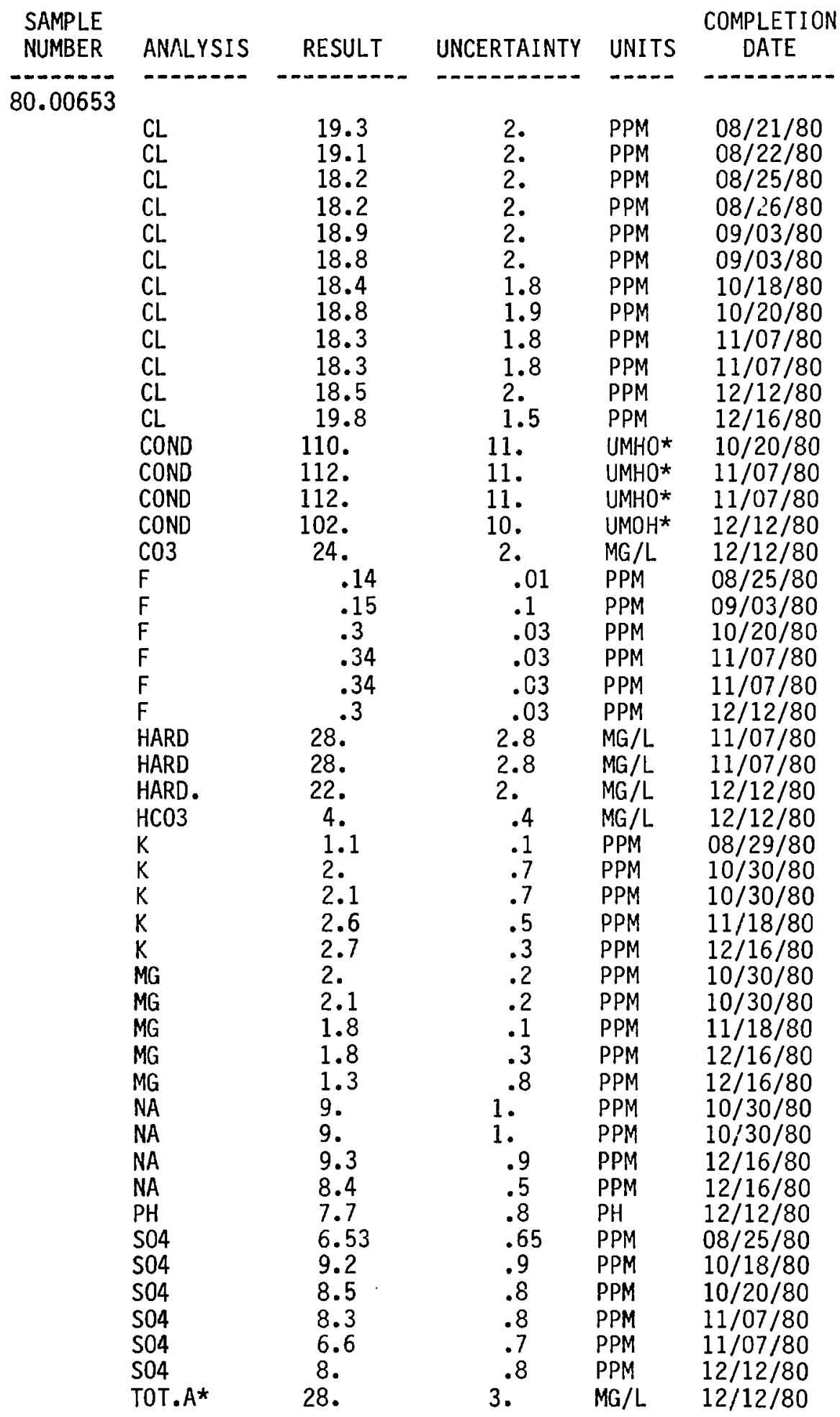


TABLE C-II (cont)

\begin{tabular}{|c|c|c|c|c|c|}
\hline $\begin{array}{l}\text { SAMPLE } \\
\text { NUMBER }\end{array}$ & ANALYSIS & RESULT & UNCERTAINTY & UNITS & $\begin{array}{c}\text { COMPLETION } \\
\text { DATE }\end{array}$ \\
\hline 8000654 & $m-\infty-m-\infty$ & $--m--\infty-m-n$ & $-\infty-\infty$ & ---- & $-m-m--n---$ \\
\hline & $\begin{array}{l}A G \\
B A\end{array}$ & $\begin{array}{r}18 . \\
180\end{array}$ & $\begin{array}{r}5 . \\
50 .\end{array}$ & $\begin{array}{l}\text { PPB } \\
\text { PPB }\end{array}$ & $\begin{array}{l}12 / 16 / 80 \\
12 / 16 / 80\end{array}$ \\
\hline 80.00655 & & & & & \\
\hline & $\begin{array}{l}A G \\
B A\end{array}$ & $\begin{array}{l}20 . \\
405 .\end{array}$ & $\begin{array}{r}5 . \\
50 .\end{array}$ & $\begin{array}{l}\text { PPB } \\
\text { PPB }\end{array}$ & $\begin{array}{l}12 / 16 / 80 \\
12 / 16 / 80\end{array}$ \\
\hline 80.00656 & & & & & \\
\hline & $\begin{array}{l}U \\
U \\
U \\
U \\
U \\
U \\
U \\
U \\
U\end{array}$ & $\begin{array}{l}150 . \\
152 \\
150 . \\
151 . \\
152 . \\
151 . \\
155 . \\
160 . \\
156 .\end{array}$ & $\begin{array}{l}10 . \\
10 . \\
10 . \\
10 . \\
10 . \\
10 . \\
15 . \\
15 . \\
15 .\end{array}$ & $\begin{array}{l}\text { PPB } \\
\text { PPB } \\
\text { PPB } \\
\text { PPB } \\
\text { PPB } \\
\text { PPB } \\
\text { PPB } \\
\text { PPB } \\
\text { PPB }\end{array}$ & $\begin{array}{l}11 / 19 / 80 \\
11 / 19 / 80 \\
11 / 19 / 80 \\
11 / 19 / 80 \\
11 / 19 / 80 \\
11 / 19 / 80 \\
12 / 30 / 80 \\
12 / 30 / 80 \\
12 / 30 / 80\end{array}$ \\
\hline
\end{tabular}




\section{APPENDIX D}

QA DATA FROM EML QA PROGRAMS 
TABLE D-I

H-8 STABLE ELEMENT DETERMINATIONS ON EML QUALITY ASSURANCE SAMPLES

\begin{tabular}{|c|c|c|c|c|c|}
\hline Element & Matrix & Date & H-8 & EML & Units \\
\hline \multirow[t]{9}{*}{$\mathrm{Cl}$} & A & $4 / 80$ & 492 & $600 \pm 12$ & $\mu \mathrm{g} / \mathrm{f}$ \\
\hline & & & 511 & & \\
\hline & & $10 / 80$ & $925 \pm 80$ & $846 \pm 51$ & \\
\hline & $W$ & $4 / 80$ & $3.7 \pm 0.4$ & $4.2 \pm 0.2$ & ppm \\
\hline & & & $4.1 \pm 0.4$ & & \\
\hline & & & $4.1 \pm 0.4$ & & \\
\hline & & $10 / 80$ & $16.8 \pm 1.7$ & $16.4 \pm 0.2$ & \\
\hline & & & $17.0 \pm 1.7$ & & \\
\hline & $\therefore$ & & $17.1 \pm 1.7$ & & \\
\hline \multirow[t]{8}{*}{$\mathrm{Cu}$} & A & $4 / 80$ & $58 \pm 3$ & 60 & $\mu \mathrm{g} / \mathrm{f}$ \\
\hline & & & $61 \pm 3$ & & \\
\hline & & & $61 \pm 3$ & & \\
\hline & & $10 / 80$ & $24.0 \pm 1.5$ & $22.8 \pm 0.4$ & \\
\hline & & & $24.7 \pm 1.5$ & & \\
\hline & w & $10 / 80$ & $2.02 \pm 0.1$ & 2.05 & ppm \\
\hline & & & $2.01 \pm 0.1$ & & \\
\hline & & & $1.98 \pm 0.1$ & & \\
\hline \multirow[t]{5}{*}{$\mathrm{Mg}$} & A & $10 / 80$ & $275 \pm 15$ & $258 \pm 10$ & $\mu \mathrm{g} / \mathrm{f}$ \\
\hline & & & $272 \pm 15$ & & \\
\hline & w & $10 / 80$ & $5.1 \pm 0.3$ & $4.99 \pm 0.05$ & ppm \\
\hline & & & $5.0 \pm 0.3$ & & \\
\hline & & & $5.2 \pm 0.3$ & & \\
\hline \multirow[t]{5}{*}{$\mathrm{Mn}$} & A & $10 / 80$ & $25.6 \pm 1.5$ & $23.8 \pm 0.7$ & $\mu \mathrm{g} / \mathrm{f}$ \\
\hline & & & $26.7 \pm 1.5$ & & \\
\hline & W & $10 / 80$ & $2.10 \pm 0.1$ & 2.05 & ppm \\
\hline & & & $2.11 \pm 0.1$ & & \\
\hline & & & $2.09 \pm 0.1$ & & \\
\hline \multirow[t]{5}{*}{$\mathrm{Na}$} & A & $10 / 80$ & $581 \pm 30$ & $561 \pm 22$ & $\mu \mathrm{g} / \mathrm{f}$ \\
\hline & & & $591 \pm 30$ & & \\
\hline & W & $10 / 80$ & $10.9 \pm 0.5$ & $10.7 \pm 0.3$ & $\mathrm{ppm}$ \\
\hline & & & $10.8 \pm 0.5$ & & \\
\hline & & & $11.0+0.5$ & & \\
\hline
\end{tabular}


TABLE D-I (cont)

\begin{tabular}{|c|c|c|c|c|c|}
\hline Element & Matrix & Date & H-8 & EML & Units \\
\hline \multirow[t]{2}{*}{$\mathrm{NO}_{3}$} & A & $10 / 80$ & $\begin{array}{l}1480 \pm 100 \\
1440 \pm 100\end{array}$ & $1340 \pm 94$ & $\mu \mathrm{g} / \mathrm{f}$ \\
\hline & $\mathbf{w}$ & $\begin{array}{r}4 / 80 \\
10 / 80\end{array}$ & $\begin{aligned} 19 & \pm 2 \\
27.5 & \pm 2.7 \\
28.3 & \pm 2.8 \\
28.4 & \pm 2.8\end{aligned}$ & $\begin{array}{r}20 \pm 0.6 \\
25.5 \pm 0.3\end{array}$ & $\mathrm{ppm}$ \\
\hline \multirow[t]{2}{*}{$\mathrm{Pb}$} & $\mathbf{w}$ & $10 / 80$ & $\begin{array}{l}2.01 \pm 0.1 \\
2.06 \pm 0.1 \\
2.06 \pm 0.1\end{array}$ & 2.05 & $\mathrm{ppm}$ \\
\hline & A & $10 / 80$ & $\begin{array}{l}25.2 \pm 1.5 \\
26.2 \pm 1.5\end{array}$ & $23.0 \pm 0.5$ & $\mu \mathrm{g} / \mathrm{f}$ \\
\hline \multirow[t]{2}{*}{$\mathrm{SO}_{4}$} & A & $10 / 80$ & $\begin{array}{l}2890 \pm 150 \\
2845 \pm 150\end{array}$ & $2670 \pm 160$ & $\mu \mathrm{g} / \mathrm{f}$ \\
\hline & w & $\begin{array}{r}4 / 80 \\
10 / 80\end{array}$ & $\begin{array}{l}46 \pm 5 \\
43 \pm 5 \\
43 \pm 5 \\
43 \pm 5\end{array}$ & $\begin{array}{c}50 \pm 2 \\
49.9 \pm 0.5\end{array}$ & $\mathrm{ppm}$ \\
\hline \multirow[t]{2}{*}{$\mathbf{Z n}$} & A & $10 / 80$ & $\begin{array}{c}58 \pm 3 \\
62 \pm 3 \\
68 \pm 3 \\
66 \pm 3 \\
25.8 \pm 1.5 \\
26.4 \pm 1.5\end{array}$ & $23.1 \pm 0.9$ & $\mu \mathrm{g} / \mathrm{f}$ \\
\hline & w & $10 / 80$ & $\begin{array}{l}2.10 \pm 0.1 \\
2.11 \pm 0.1 \\
2.12 \pm 0.1\end{array}$ & 2.05 & $\mathrm{ppm}$ \\
\hline
\end{tabular}


TABLE D-II

H-8 RADIOCHEMICAL DETERMINATION ON EML QUALITY ASSURANCE SAMPLES

\begin{tabular}{|c|c|c|c|c|c|}
\hline Nuclide & Matrix & Date & $\mathrm{H}-\mathbf{8}$ & EML & Units \\
\hline${ }^{3} \mathrm{H}$ & $\mathbf{w}$ & $\begin{array}{r}4 / 80 \\
10 / 80\end{array}$ & $\begin{array}{ll}10 & 000 \pm 500 \\
10 & 100 \pm 300 \\
11 & 500 \pm 300 \\
11 & 800 \pm 300\end{array}$ & $\begin{array}{l}10300 \pm 200 \\
14900 \pm 150\end{array}$ & $\mathrm{pCi} / \ell$ \\
\hline${ }^{40} \mathrm{~K}$ & $\mathbf{S}$ & $10 / 80$ & $\begin{array}{l}23 \pm 2 \\
21 \pm 2\end{array}$ & $20.7 \pm 1.0$ & $\mathrm{pCi} / \mathrm{g}$ \\
\hline${ }^{60} \mathrm{Co}$ & w & $10 / 80$ & $\begin{array}{l}1940 \pm 100 \\
1920 \pm 100\end{array}$ & $1970 \pm 100$ & $\mathrm{pCi} / \ell$ \\
\hline & $\mathbf{S}$ & $10 / 80$ & $\begin{array}{l}0.2 \pm 0.07 \\
0.7 \pm 0.06\end{array}$ & $0.1 \pm 0.007$ & $\mathrm{pCi} / \mathrm{g}$ \\
\hline${ }^{90} \mathrm{Sr}$ & v & $\begin{array}{r}4 / 80 \\
10 / 80\end{array}$ & $\begin{array}{r}27.4 \pm 1.0 \\
8.6 \pm 0.2 \\
9.6 \pm 0.4\end{array}$ & $\begin{array}{l}24.6 \pm 0.5 \\
13.8 \pm 1.0\end{array}$ & $\mathrm{pCi} / \mathrm{g}$ \\
\hline & B & $\begin{array}{r}4 / 80 \\
10 / 80\end{array}$ & $\begin{array}{l}13.9 \pm 0.3 \\
33.8 \pm 1.3 \\
36.0 \pm 1.4\end{array}$ & $\begin{array}{c}18.2 \pm 0.1 \\
38.7 \pm 3.5\end{array}$ & $\mathrm{pCi} / \mathrm{g}$ \\
\hline & $\mathbf{s}$ & $\begin{array}{r}4 / 80 \\
10 / 80\end{array}$ & $\begin{array}{l}0.68 \pm 0.16 \\
0.70 \pm 0.23\end{array}$ & $\begin{array}{l}0.37 \pm 0.01 \\
0.46 \pm 0.01\end{array}$ & $\mathrm{pCi} / \mathrm{g}$ \\
\hline${ }^{134} \mathrm{Cs}$ & $\mathbf{w}$ & $10 / 80$ & $\begin{array}{l}2590 \pm 140 \\
2640 \pm 140\end{array}$ & $2440 \pm 100$ & $\mathrm{pCi} / \ell$ \\
\hline${ }^{137} \mathrm{Cs}$ & $\mathbf{w}$ & $\begin{array}{r}4 / 80 \\
10 / 80\end{array}$ & $\begin{array}{l}1040 \pm 60 \\
2310 \pm 120 \\
2280 \pm 120\end{array}$ & $\begin{array}{r}978 \pm 20 \\
2260 \pm 90\end{array}$ & $\mathrm{pCi} / \ell$ \\
\hline & $\mathbf{v}$ & $4 / 80$ & $21.3 \pm 1.3$ & $17.1 \pm 0.2$ & $\mathrm{pCi} / \mathrm{g}$ \\
\hline & B & $4 / 80$ & $14.2 \pm 1.0$ & $12.2 \pm 0.2$ & $\mathrm{pCi} / \mathrm{g}$ \\
\hline & $\mathbf{s}$ & $\begin{array}{r}4 / 80 \\
10 / 80\end{array}$ & $\begin{array}{r}7.8 \pm 0.3 \\
13.0 \pm 0.5 \\
13.0 \pm 0.5\end{array}$ & $\begin{array}{r}6.8 \pm 0.1 \\
11.0 \pm 0.6\end{array}$ & $\mathrm{pCi} / \mathrm{g}$ \\
\hline
\end{tabular}


TABLE D-II (cont)

\begin{tabular}{|c|c|c|c|c|c|}
\hline Nuclide & Matrix & Date & H-8 & EML & Units \\
\hline${ }^{226} \mathbf{R a}$ & $\mathbf{s}$ & $10 / 80$ & $\begin{array}{l}0.53 \pm 0.06 \\
0.56 \pm 0.012\end{array}$ & $0.66 \pm 0.07$ & $\mathrm{pCi} / \mathrm{g}$ \\
\hline \multirow[t]{3}{*}{${ }^{238} \mathrm{Pu}$} & $\mathbf{W}$ & $10 / 80$ & $<0.016$ & 0 & $\mathrm{pCi} / \ell$ \\
\hline & $\mathrm{s}$ & $10 / 80$ & $\begin{array}{l}0.004 \pm 0.002 \\
0.002 \pm 0.001 \\
0.001 \pm 0.006\end{array}$ & $0.0038 \pm 0.0003$ & $\mathrm{pCi} / \mathrm{g}$ \\
\hline & B & $10 / 80$ & $\begin{array}{l}0.015 \pm 0.002 \\
0.016 \pm 0.002\end{array}$ & $0.015 \pm 0.002$ & $\mathrm{pCi} / \mathrm{g}$ \\
\hline \multirow[t]{5}{*}{${ }^{239} \mathrm{Pu}$} & $\mathbf{w}$ & $\begin{array}{r}4 / 80 \\
10 / 80\end{array}$ & $\begin{array}{l}0.08 \pm 0.04 \\
4.06 \pm 0.17\end{array}$ & $\begin{array}{l}\text { none.reported } \\
5.77 \pm 0.69\end{array}$ & $\mathrm{pCi} / \ell$ \\
\hline & $\mathbf{s}$ & $\begin{array}{r}4 / 80 \\
10 / 80\end{array}$ & $\begin{array}{l}0.056 \pm 0.006 \\
0.099 \pm 0.008 \\
0.125 \pm 0.008 \\
0.100 \pm 0.005\end{array}$ & $\begin{array}{l}0.056 \pm 0.006 \\
0.090 \pm 0.009\end{array}$ & $\mathrm{pCi} / \mathrm{g}$ \\
\hline & B & $\begin{array}{r}4 / 80 \\
10 / 80\end{array}$ & $\begin{array}{l}0.062 \pm 0.005 \\
0.154 \pm 0.007 \\
0.178 \pm 0.008\end{array}$ & $\begin{array}{c}0.0775 \pm 0.0016 \\
0.180 \pm 0.031\end{array}$ & $\mathrm{pCi} / \mathrm{g}$ \\
\hline & A & $10 / 80$ & $2.11 \pm 0.09$ & $2.46 \pm 0.20$ & $\mathrm{pCi} / \mathrm{f}$ \\
\hline & V & $10 / 80$ & $\begin{array}{l}0.061 \pm 0.008 \\
0.055 \pm 0.006\end{array}$ & $0.063 \pm 0.005$ & $\mathrm{pCi} / \mathrm{g}$ \\
\hline \multirow[t]{4}{*}{${ }^{241} \mathrm{Am}$} & $\mathbf{S}$ & $\begin{array}{r}4 / 80 \\
10 / 80\end{array}$ & $\begin{array}{l}0.042 \pm 0.005 \\
0.054 \pm 0.002 \\
0.044 \pm 0.002 \\
0.040 \pm 0.002\end{array}$ & $\begin{array}{c}0.024 \pm 0.002 \\
0.0436 \pm 0.0022\end{array}$ & $\mathrm{pCi} / \mathrm{g}$ \\
\hline & V & $4 / 80$ & $0.045 \pm 0.005$ & $0.040 \pm 0.004$ & $\mathrm{pCi} / \mathrm{g}$ \\
\hline & B & $4 / 80$ & $0.034 \pm 0.004$ & $0.0239 \pm 0.0055$ & $\mathrm{pCi} / \mathrm{g}$ \\
\hline & $\mathbf{W}$ & $\begin{array}{r}4 / 80 \\
10 / 80\end{array}$ & $\begin{array}{l}7.0 \pm 0.05 \\
3.2 \pm 1.5\end{array}$ & $\begin{array}{c}7.9 \pm 0.5 \\
4.86 \pm 0.58\end{array}$ & $\mathrm{pCi} / \ell$ \\
\hline Total U & $\mathbf{W}$ & $4 / 80$ & $30 \pm 3$ & $28.3 \pm 4.0$ & $\mathrm{ppb}$ \\
\hline
\end{tabular}


APPENDIX E

QA DATA FROM EPA QA PROGRAMS 
TABLE E-I

RADIOCHEMICAL DETERMINATIONS ON EPA QUALITY ASSURANCE WATER SAMPLES (pCi/ )

\begin{tabular}{|c|c|c|c|c|c|c|c|}
\hline Nuclide & Date & H-8 & EPA & Nuclide & Date & H-8 & EPA \\
\hline \multirow[t]{25}{*}{ Gross Alpha } & $1 / 80$ & $33 \pm 7$ & $30 \pm 8$ & & $9 / 80$ & $20 \pm 2$ & $21 \pm 5$ \\
\hline & & $30 \pm 6$ & & & & $21 \pm 2$ & \\
\hline & & $33 \pm 7$ & & & & $25 \pm 3$ & \\
\hline & $3 / 80$ & $13 \pm 3$ & $13 \pm 5$ & & $10 / 80$ & $63 \pm 6$ & 60 \\
\hline & & $22 \pm 5$ & & & & $64 \pm 7$ & \\
\hline & & $19 \pm 4$ & & & & $64 \pm 7$ & \\
\hline & $4 / 80$ & $500 \pm 100$ & $98 \pm 24$ & & $11 / 80$ & $14.7 \pm 1.6$ & $13 \pm 5$ \\
\hline & & $460 \pm 90$ & & & & $14.8 \pm 1.6$ & \\
\hline & & $450 \pm 90$ & & & & $14.2 \pm 1.6$ & \\
\hline & $5 / 80$ & $26 \pm 5$ & $23 \pm 5$ & & & & \\
\hline & & $26 \pm 5$ & & ${ }^{3} \mathrm{H}$ & $2 / 80$ & $1900 \pm 300$ & $1750 \pm 341$ \\
\hline & & $23 \pm 5$ & & & $4 / 80$ & $3300 \pm 200$ & $3400 \pm 360$ \\
\hline & $7 / 80$ & $39 \pm 8$ & $36 \pm 9$ & & & $3700 \pm 200$ & \\
\hline & & $46 \pm 9$ & & & $6 / 80$ & $2100 \pm 200$ & $2000 \pm 345$ \\
\hline & & $43 \pm 9$ & & & & $1700 \pm 200$ & \\
\hline & $9 / 80$ & $26 \pm 5$ & $32 \pm 8$ & & & $1900 \pm 200$ & \\
\hline & & $30 \pm 6$ & & & $8 / 80$ & $900 \pm 200$ & $1210 \pm 329$ \\
\hline & & $28 \pm 6$ & & & & $1400 \pm 200$ & \\
\hline & $10 / 80$ & $37 \pm 8$ & 39 & & & $1200 \pm 200$ & \\
\hline & & $51 \pm 10$ & & & $10 / 80$ & $1800 \pm 200$ & $3200 \pm 360$ \\
\hline & & $51 \pm 10$ & & & & $3000 \pm 200$ & \\
\hline & $11 / 80$ & $11 \pm 2$ & $16 \pm 5$ & & & $3000 \pm 200$ & \\
\hline & & $16 \pm 3$ & & & $12 / 80$ & $1900 \pm 200$ & $2240 \pm 350$ \\
\hline & & $12 \pm 3$ & & & & $2200 \pm 200$ & \\
\hline & & & & & & $2000 \pm 200$ & \\
\hline \multirow[t]{15}{*}{ Gross Beta } & $1 / 80$ & $50 \pm 5$ & $45 \pm 5$ & & & & \\
\hline & & $46 \pm 5$ & & ${ }^{51} \mathrm{Cr}$ & $2 / 80$ & $<200$ & $101 \pm 5$ \\
\hline & & $43 \pm 5$ & & & & $<200$ & \\
\hline & $3 / 80$ & $27 \pm 3$ & $22 \pm 5$ & & & $<500$ & \\
\hline & & $31 \pm 3$ & & & $6 / 80$ & $<360$ & $13 \pm 5$ \\
\hline & & $31 \pm 4$ & & & & $<390$ & \\
\hline & $4 / 80$ & $300 \pm 30$ & $100 \pm 5$ & & & $<450$ & \\
\hline & & $310 \pm 30$ & & & $10 / 80$ & $<150$ & $86 \pm 5$ \\
\hline & & $300 \pm 30$ & & & & $<150$ & \\
\hline & $5 / 80$ & $18 \pm 2$ & $14 \pm 5$ & & & $<150$ & \\
\hline & & $20 \pm 2$ & & & & & \\
\hline & & $18 \pm 2$ & & ${ }^{60} \mathrm{Co}$ & $2 / 80$ & $<30$ & $11 \pm 5$ \\
\hline & $7 / 80$ & $47 \pm 5$ & $38 \pm 5$ & & & $<11$ & \\
\hline & & $50 \pm 5$ & & & & $<30$ & \\
\hline & & $48 \pm 5$ & & & & & \\
\hline
\end{tabular}


TABLE E-I (cont)

\begin{tabular}{|c|c|c|c|c|c|c|c|}
\hline Nuclide & Date & H-8 & EPA & Nuclide & Date & H-8 & EPA \\
\hline & $6 / 30$ & $<30$ & $5 \pm 5$ & & $10 / 80$ & $<170$ & 0 \\
\hline & & $<36$ & & & & $<170$ & \\
\hline & & $<42$ & & & & $<170$ & \\
\hline & $10 / 80$ & $<25$ & $16 \pm 5$ & & & & \\
\hline & & $<25$ & & ${ }^{134} \mathrm{Cs}$ & $2 / 80$ & $<30$ & $10 \pm 5$ \\
\hline & & $<25$ & & & & $<12$ & \\
\hline & $10 / 80$ & $50 \pm 26$ & 12 & & & $<20$ & \\
\hline & & $<23$ & & & $6 / 80$ & $<33$ & $11 \pm 5$ \\
\hline & & $<22$ & & & & $<36$ & \\
\hline & & & & & & $<42$ & \\
\hline \multirow[t]{13}{*}{${ }^{{ }^{s}} \mathrm{Zn}$} & $2 / 80$ & $<100$ & $25 \pm 5$ & & $10 / 80$ & $57 \pm 13$ & $20 \pm 5$ \\
\hline & & $<40$ & & & & $19 \pm 14$ & \\
\hline & & $<80$ & & & & $25 \pm 22$ & \\
\hline & $6 / 80$ & $<90$ & $23 \pm 5$ & & $10 / 80$ & $<22$ & 12 \\
\hline & & $<120$ & & & & $<22$ & \\
\hline & & $<120$ & & & & $<22$ & \\
\hline & $10 / 80$ & $<50$ & $25 \pm 5$ & & & & \\
\hline & & $<50$ & & ${ }^{137} \mathrm{Cs}$ & $2 / 80$ & $80 \pm 40$ & $30 \pm 5$ \\
\hline & & $<50$ & & & & $36 \pm 12$ & \\
\hline & $10 / 80$ & $<40$ & $\mathbf{0}$ & & & $<30$ & \\
\hline & & $<4$ & & & $6 / 80$ & $<30$ & $17 \pm 5$ \\
\hline & & $<4$ & & & & $<33$ & \\
\hline & & & & & & $<39$ & \\
\hline \multirow[t]{10}{*}{${ }^{90} \mathrm{Sr}$} & $2 / 80$ & $19 \pm 1$ & $20 \pm 1.5$ & & $10 / 80$ & $<30$ & $12 \pm 5$ \\
\hline & & $20 \pm 1$ & & & & $<30$ & \\
\hline & & $20 \pm 1$ & & & & $<30$ & \\
\hline & $9 / 80$ & $12.4 \pm 0.6$ & $15 \pm 1.5$ & & $10 / 80$ & $5 \pm 16$ & 20 \\
\hline & & $13.1 \pm 0.8$ & & & & $33 \pm 21$ & \\
\hline & & $16.2 \pm 0.8$ & & & & $45 \pm 20$ & \\
\hline & $10 / 80$ & $0.8 \pm 0.2$ & 0 & & & & \\
\hline & & $<0.3$ & & ${ }^{226} \mathbf{R a}$ & $10 / 80$ & $10.3 \pm 1.0$ & 12.8 \\
\hline & & $0.4 \pm 0.2$ & & & & $11.0 \pm 1.1$ & \\
\hline & & & & & & $12.5 \pm 1.2$ & \\
\hline \multirow[t]{9}{*}{${ }^{106} \mathrm{Ru}$} & $2 / 80$ & $<300$ & $51 \pm 5$ & & & & \\
\hline & & $290 \pm 100$ & & ${ }^{239} \mathrm{Pu}$ & $1 / 80$ & $3.26 \pm 0.14$ & $3.39 \pm 0.34$ \\
\hline & & $<200$ & & & & $3.14 \pm 0.16$ & \\
\hline & $6 / 80$ & $<270$ & $37 \pm 5$ & & $5 / 80$ & $6.0 \pm 0.2$ & $8 \pm 1$ \\
\hline & & $<300$ & & & & $5.8 \pm 0.2$ & \\
\hline & & $<360$ & & & & $6.2 \pm 0.2$ & \\
\hline & $10 / 80$ & $<120$ & $46 \pm 5$ & & $7 / 80$ & $3.22 \pm 0.18$ & $4.49 \pm 0.45$ \\
\hline & & $<120$ & & & & $3.72 \pm 0.17$ & \\
\hline & & $<120$ & & & & $3.46 \pm 0.14$ & \\
\hline
\end{tabular}


TABLE E-1 (cont)

\begin{tabular}{|c|c|c|c|c|c|c|c|}
\hline Nuclide & Date & H-8 & EPA & Nuclide & Date & H-8 & EPA \\
\hline & $11 / 80$ & $\begin{array}{l}6.1 \pm 0.2 \\
6.0 \pm 0.2 \\
6.4 \pm 0.2\end{array}$ & $9.1 \pm 0.9$ & & $8 / 80$ & $\begin{array}{l}24 \pm 3 \\
26 \pm 3 \\
24 \pm 3\end{array}$ & $27 \pm 3$ \\
\hline $\mathbf{U}$ & $2 / 80$ & $\begin{array}{l}33 \pm 3 \\
33 \pm 3 \\
34 \pm 3\end{array}$ & $32 \pm 4$ & & $10 / 80$ & $\begin{array}{l}5.3 \pm 0.5 \\
4.0 \pm 0.4 \\
3.8 \pm 0.4\end{array}$ & $5 \pm 1$ \\
\hline
\end{tabular}

TABLE E-II

H-8 RADIOCHEMICAL DETERMINATIONS ON EPA QUALITY ASSURANCE AIR FILTER SAMPLES (pCi/filter)

\begin{tabular}{|c|c|c|c|}
\hline Nuclide & Date & H-8 & EPA \\
\hline \multirow[t]{2}{*}{ Gross Alpha } & $6 / 80$ & $\begin{array}{l}30 \pm 7 \\
28 \pm 6 \\
29 \pm 6\end{array}$ & $24 \pm 6$ \\
\hline & $12 / 80$ & $\begin{array}{l}26 \pm 3 \\
26 \pm 3 \\
27 \pm 3 \\
27 \pm 6 \\
23 \pm 5 \\
23 \pm 5\end{array}$ & $24 \pm 5$ \\
\hline \multirow[t]{3}{*}{ Gross Beta } & $6 / 80$ & $\begin{array}{l}33 \pm 4 \\
34 \pm 4 \\
38 \pm 5\end{array}$ & $28 \pm 5$ \\
\hline & $9 / 80$ & $\begin{array}{l}12.8 \pm 1.4 \\
13.8 \pm 1.5 \\
13.4 \pm 1.5\end{array}$ & $10 \pm 5$ \\
\hline & $12 / 80$ & $\begin{array}{l}25 \pm 3 \\
23 \pm 3 \\
22 \pm 3\end{array}$ & $19 \pm 3$ \\
\hline${ }^{00} \mathrm{Sr}$ & $3 / 80$ & $\begin{array}{l}<0.2 \\
1.0 \pm 0.2 \\
2.7 \pm 0.2\end{array}$ & $10 \pm 1.5$ \\
\hline
\end{tabular}


TABLE E-III

H-8 RADIOCHEMICAL DETERMINATIONS ON EPA QUALITY ASSURANCE FOOD SAMPLES $(\mathrm{pCi} / \mathrm{kg})$

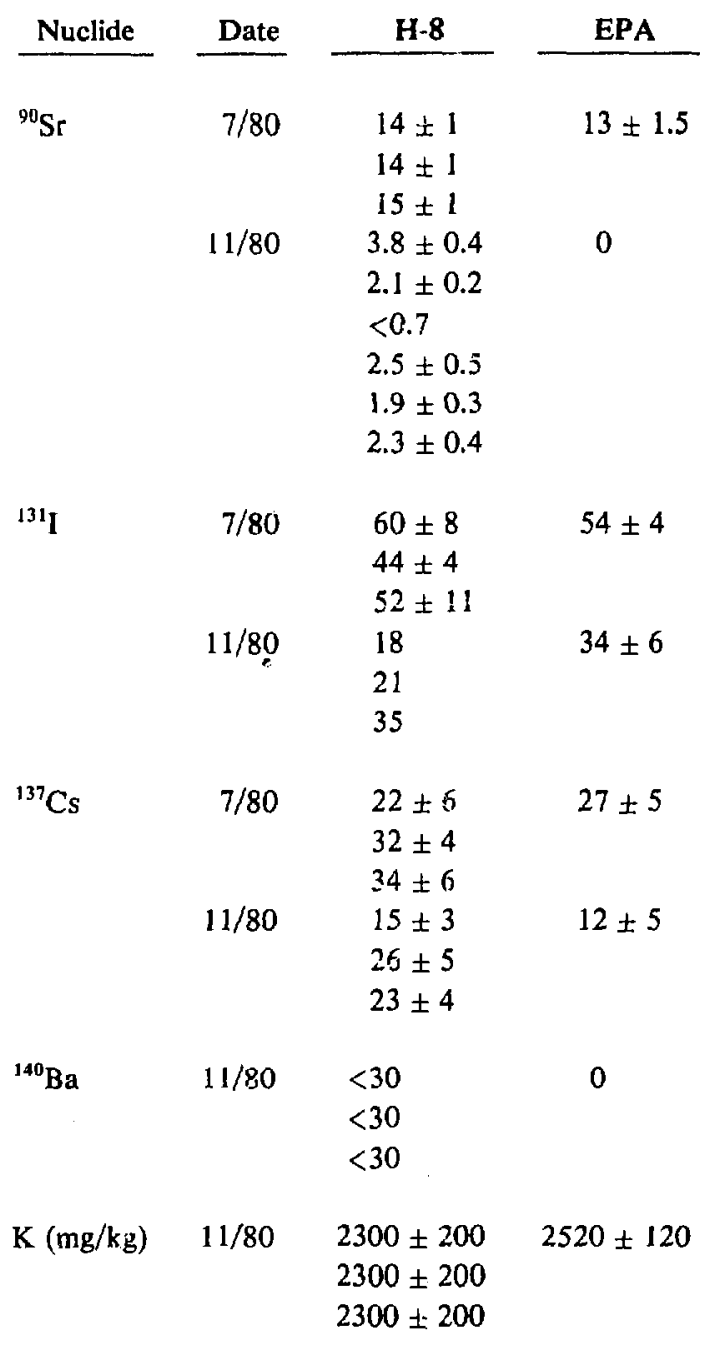

